\title{
Investigating the anti-cocaine, anti-nociceptive properties and side effects of MP1104, a novel mixed opioid receptor agonist
}

\section{Diana Vivian Atigari}

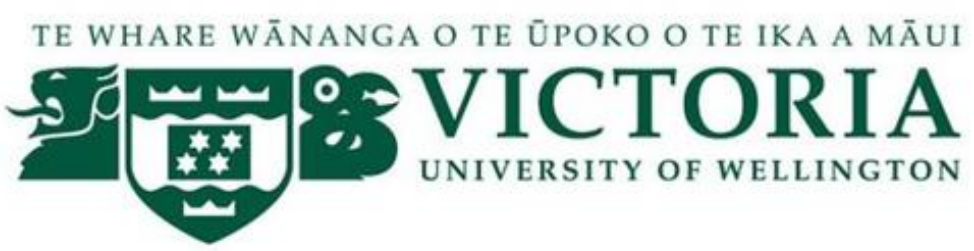

School of Biological Sciences

Te kura Matauranga Koiora

A thesis submitted to the Victoria University of Wellington in fulfilment of the requirements for the degree of Doctor of Philosophy in Biomedical Science 


\section{Abstract}

Rationale: Drug addiction is a chronic, relapsing disease with great socioeconomic and morbidity costs. There are limited treatments, with no Food and Drug Administration approved pharmacotherapies available for psychostimulant addiction. In addition, the use of prescription opioid medications has reached epidemic proportions in the world. More than 40,000 deaths from prescription opioid overdose was reported in USA alone in the year 2017. There is an urgent need for the development of effective, non-addictive pain medications and addiction treatments. The opioid receptors play an important role in the modulation of pain and addiction. Mu opioid receptor (MOPr) agonists are widely used to treat pain, however, can also induce respiratory depression, tolerance and addiction. In contrast, drugs activating the kappa opioid receptor (KOPr) attenuate the rewarding properties of drugs, hence are promising non-addictive analgesics. However, side effects like aversion, sedation, anxiety and depression limit their clinical utility. Delta opioid receptor (DOPr) agonists have rewarding, anti-nociceptive and antidepressive properties, but can also cause seizures. We hypothesise that development of mixed opioid receptor ligands may have therapeutic properties with reduced side effects. Therefore, this thesis evaluated MP1104, a potent mixed opioid receptor agonist, with full efficacy at all three receptors and 3- and 13-fold higher binding affinity for KOPr compared to MOPr and DOPr, respectively. MP1104 was evaluated for the ability to modulate cocaine-induced behaviours, the anti-nociceptive effects and side effects.

Methods: Male Sprague-Dawley rats were used to investigate the effects of acute MP1104 treatment on cocaine self-administration and drug seeking behaviour. To determine the mechanism, the modulatory effect of MP1104 on dopamine transporter (DAT) function was assessed using rotating disk electrode voltammetry to measure dopamine uptake in rat dorsal striatum (dStr) and nucleus accumbens (NAc) tissue. Evaluation of side effects included sedation (spontaneous locomotor activity), anxiety (elevated plus maze (EPM)), aversion (conditioned place aversion (CPA)) and depression (forced swim tests (FST)) in rats. The anti-nociceptive effects were measured in the warm-water tail withdrawal assay in rats and male C57BL/6 mice. Acute and chronic administration of MP1104 were evaluated in the paclitaxel-induced neuropathic pain model in mice. 
Results: In rats trained to self-administer cocaine, acute MP1104 (0.3 and $1 \mathrm{mg} / \mathrm{kg}$, i.p.) administration reduced cocaine-primed reinstatement of drug seeking behaviour and caused a significant downward shift in the cocaine dose-response curve. The anti-cocaine effects exerted by MP1104 are in part due to increased dopamine uptake by DAT in the NAc, which was KOPrmediated.

In the warm-water tail withdrawal assay in rats, acute administration of MP1104 (0.3 and 0.6 $\mathrm{mg} / \mathrm{kg}$, i.p.) was 4 times longer acting $(8 \mathrm{~h})$ than morphine $(2 \mathrm{~h})$. These effects were both $\mathrm{KOPr}$ and DOPr dependent. In the dose-response tail withdrawal assay, MP1104 was found to be potent in both rats $\left(E D_{50}=0.58 \mathrm{mg} / \mathrm{kg}\right.$, s.c.) and mice $\left(E_{50}=0.35 \mathrm{mg} / \mathrm{kg}\right.$, s.c.). In the paclitaxel-induced neuropathic pain model, mice treated with MP1104 showed potent reductions in both mechanical $\left(E D_{50}=0.449 \mathrm{mg} / \mathrm{kg}\right.$, s.c.) and cold $\left(E D_{50}=0.479 \mathrm{mg} / \mathrm{kg}\right.$, s.c.) allodynia compared to morphine. Following chronic daily administration of the $\mathrm{ED}_{80}$ dose, MP1104 (1.2 mg/kg, i.p.) was more potent than morphine in reducing mechanical and cold allodynia. Surprisingly, MP1104 reversed responding back to baseline (non-disease) levels. The most remarkable finding was that MP1104, unlike morphine did not produce tolerance when administered chronically. When the side effects of MP1104 were evaluated in rats, no significant anxiogenic effects were seen in the EPM, nor pro-depressive effects in the FST, nor aversion in CPA tests in rats. Furthermore, pretreatment with a DOPr antagonist, led to MP1104 producing aversive effects. This data suggests that the DOPr agonist actions of MP1104 attenuate the KOPr-mediated aversive effects of MP1104. However, at higher doses, MP1104 (1 mg/kg, i.p.) was found to be sedative.

Conclusions: MP1104 exerts potent anti-cocaine properties in self-administration tests. The reduced cocaine reward is at least in part due to the ability of MP1104 to modulate DAT function by increasing dopamine uptake in the NAc. MP1104 is also a potent and long-lasting antinociceptive agent in rats. Significantly, when evaluated in a chronic neuropathic pain model, MP1104 was potent with no tolerance to the anti-nociceptive effects observed. Moreover, MP1104 showed fewer side effects with reduced sedative effects and no observed anxiety, aversive, nor pro-depressive effects, unlike pure KOPr agonists.

This data supports the therapeutic development of mixed opioid receptor agonists, particularly mixed KOPr/DOPr agonists as non-addictive pain medications and anti-cocaine pharmacotherapies with fewer side effects. 


\section{Acknowledgments}

This journey would not have been possible without the support and encouragement of many people to whom I am very grateful.

I thank my Lord and Saviour Jesus Christ, for His guidance for each day and for the grace in helping me finish my PhD study successfully.

I would like to express my sincere gratitude to my supervisor Dr Bronwyn Kivell. Thank you for the continuous support of my PhD study, for your patience, motivation, and immense knowledge. Your mentoring helped me in all the time of research and writing of this thesis. Thank you for trusting me with this project and supporting me throughout these years. My sincere thank you to my secondary supervisor, Prof John Miller, for his support and insightful comments. I also thank the rest of my PhD committee, Dr Darren Day and Dr Melanie McConnell, for their valuable comments.

I greatly appreciate the support received from Victoria University of Wellington, thank you for your warm welcome. I would also like to thank Victoria University of Wellington Research Fund committee and Wellington Medical Research Foundation for providing funding for this project. In addition, to the Research for life, the New Zealand Society for Biochemistry and Molecular Biology (NZSBMB) and Victoria University Faculty Strategic Research Grants for providing funding to travel to International conferences to present my research findings there. I especially thank Dr Monica Gerth for her support with NZSBMB travel award.

I am thankful to our collaborator Dr Susruta Majumdar for his valuable feedback, encouragement and providing us with MP1104 compound.

I acknowledge the enormous support I received from Dr Amy Ewald at the start of my project. Thank you very much for your guidance and patience. I would also like to thank David Young for being there to assist me during the initials days of my research. Thank you for your willingness to help. Thank you Aimee C for help with CPA and help with animal handling skills. I take this opportunity to thank all my fellow labmates. Thank you Dr Kelly Paton for teaching me everything I needed to know regarding pain research, I am extremely grateful for your suggestions and advice. Special thanks to Dr Andy and Amy Alder. I am indebted to you both for your help with teaching and assisting me with things I needed to know. Thank you Stephen for all the support and constant readiness to provide help. You are amazing. I will always cherish the moments we 
spent in the rat room (Kirk building), cleaning, washing cages and having wonderful faith oriented conversations. I am also very grateful to Dr Nikki and Kendra for always willing to offer help and to Afnan for the laughs and giggles. I extend my heartfelt gratitude to the other lab group members who have shared this wonderful journey with me: Janine, Adam, Susan, Miguel, Jasleen, Sam, Phyllida, Rickii, Isobel, Harvir, Catie, Zinmar, Lisa and Chrysta. Also, thank you Dr Fabian Westermann for your valuable advices and guidance during my PhD journey.

I appreciate the help I received from technicians within the SBS for making and fixing equipment when required. Thank you Stephen, Neville, Craig, Shaun, Sushila, Derek, Ange, Lesley, Pisana, Adrian, Chris, and a special thank you to Paul for help with RDEV equipment setup. Also, thank you to all the SBS administration staff for your warm welcome and support. Thank you Mary, Lesley, Mark, Paul, Sandra, Charlotte and Sonja. Special acknowledgment to Dr Anne Macaskill and Al Abenoja for assistance with scripting programme for dose-response cocaine selfadministration protocol and to Danyl Mclauchlan for technical/ITS assistance with RDEV and drug self-administration experiments.

I especially thank Patricia Stein for all the help she provided during the final stages of my PhD. Thank you for understanding my situation and offering great support.

I am indebted to few families who have greatly supported me from the day I moved to Wellington. Thank you Pastor Baskaran Robert (anna) (especially for your spiritual counselling and guidance), Christina akka and Aaris for your love and encouragement. Thank you Arvind anna, Lydia akka (particluarly for preparing delicious meals for us), Adia and Abhi for your affection and support. Thank you to my Church (The Ekkaallam Church) members and Prison fellowship of NZ friends for your continuous prayers and encouragement. I was fortunate to make good friends who put up with me sharing my rants and successes. Thank you Tulsi (for your constant love and prayers for me), Jamie and Riya.

To my family, thank you Dad, Mum and Granny for the immense support, for believing in me and encouraging me to pursue my dreams. Thank you for your never-ending prayers for success in my life. To my sisters, Betty and Tabitha, who always inspire me. Thank you for your words of encouragement, tremendous support and love. To my brother Suresh, for always being there for me and motivating me. Finally to my nieces Faith, Hope and nephew Jayden for cheering me up with your cute conversations and drawings. 


\section{Table of contents}

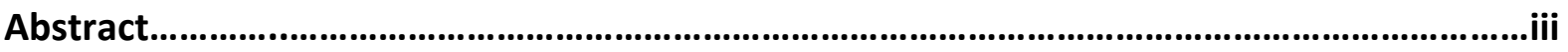

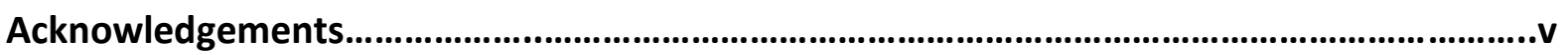

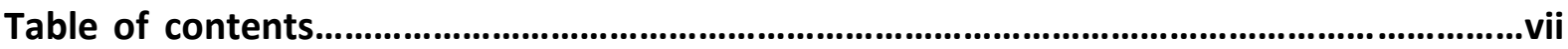

List of Figures............................................................................................................................................

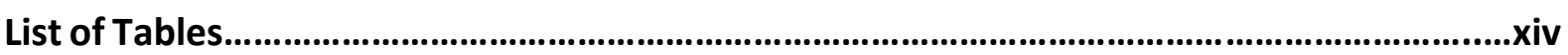

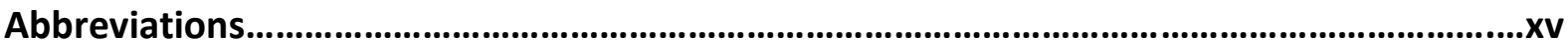

Chapter 1: Introduction ..................................................................................................................1

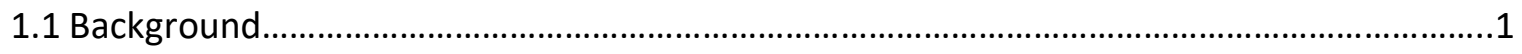

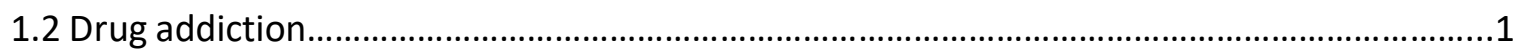

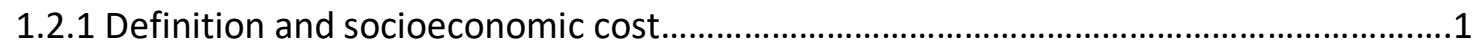

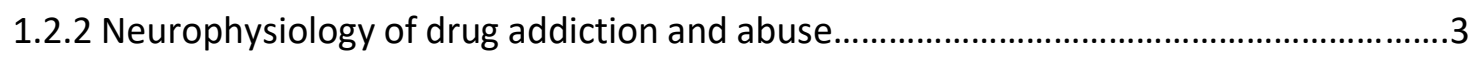

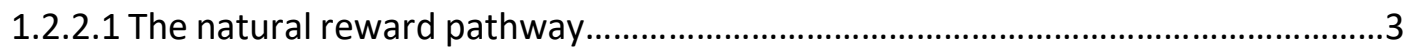

1.2.2.2 Role of dopamine in regulating drug addiction.....................................................

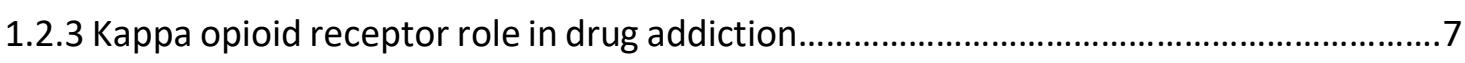

1.2.3.1 Regulation of the reward pathway by the kappa opioid receptor system............7

1.2.3.2 Regulation of dopamine transporter function by kappa opioid receptor..............8

1.2.3.3 Anti-cocaine of traditional kappa opioid receptor agonists.................................12

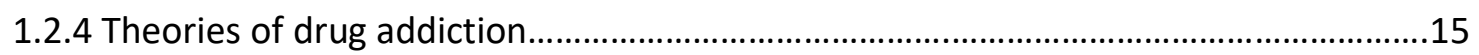

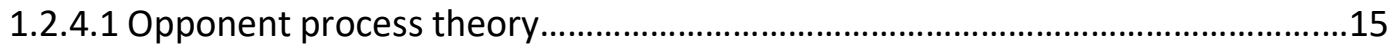

1.2.4.2 Incentive sensitisation theory............................................................................. 16

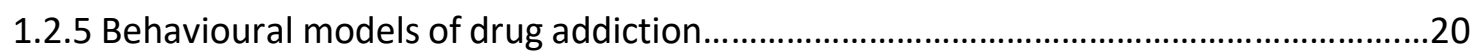

1.2.5.1 Drug self-administration: Model of drug addiction............................................20

1.2.5.2 Modelling different aspects of addiction and drug-taking behaviours pre-

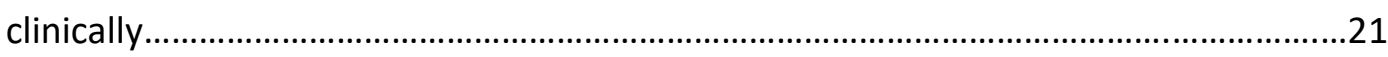

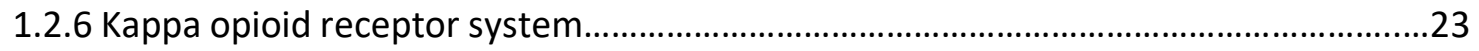

1.2.6.1 The endogenous kappa opioid receptor system...................................................23

1.2.6.2 Kappa opioid receptor signalling pathways.........................................................24 
1.2.6.3 Functional selectivity at the kappa opioid receptor

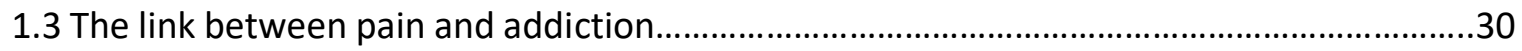

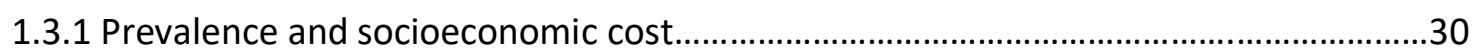

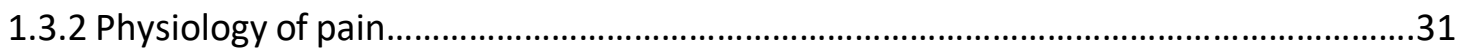

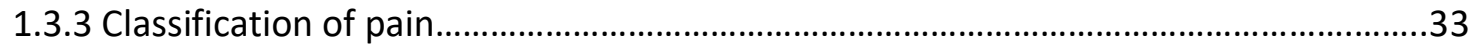

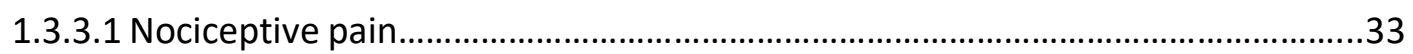

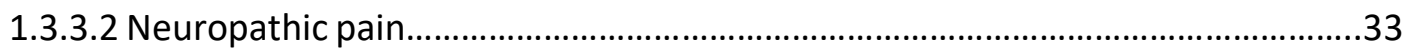

1.3.4 Kappa opioid receptor agonists for pain treatment........................................................34

1.4 Side effects of traditional kappa opioid receptor agonists.................................................38

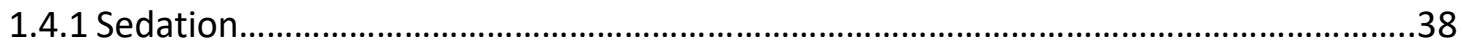

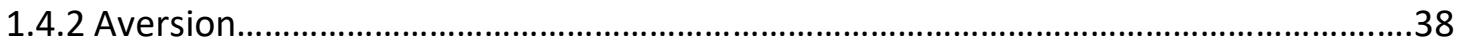

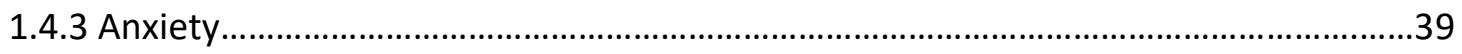

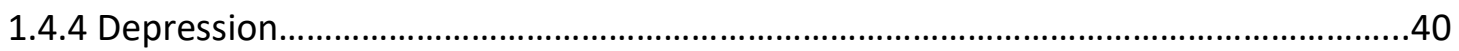

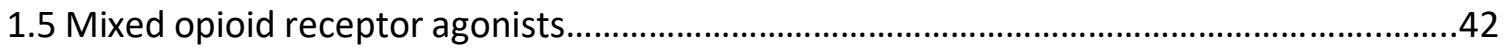

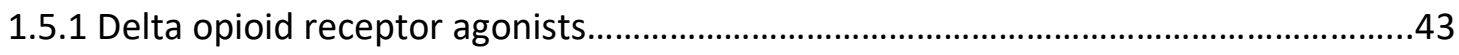

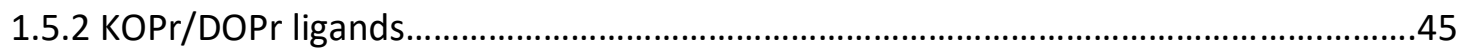

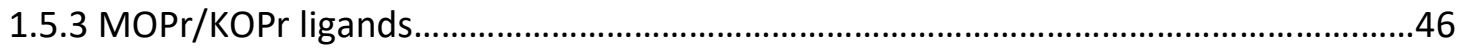

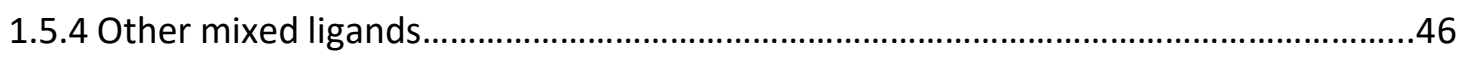

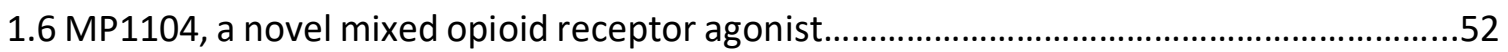

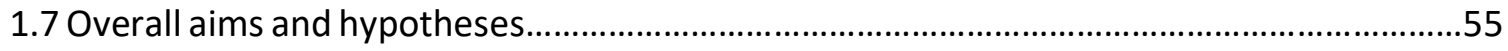

Chapter 2: Materials and methods................................................................................................57

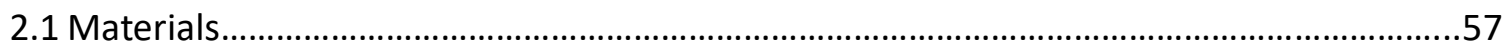

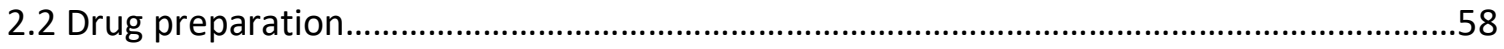

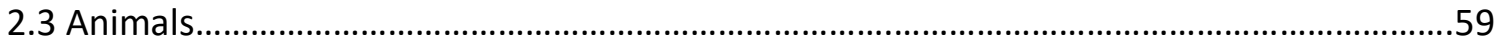

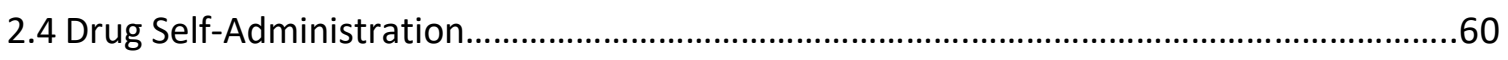

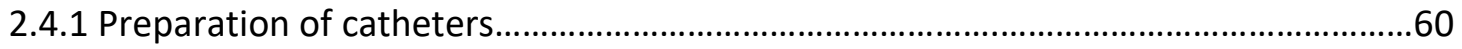

2.4.2 Surgery

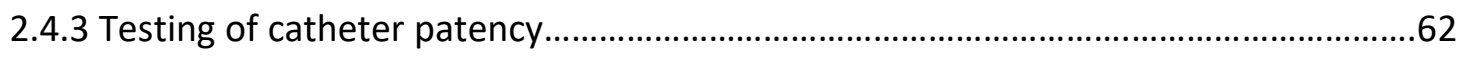

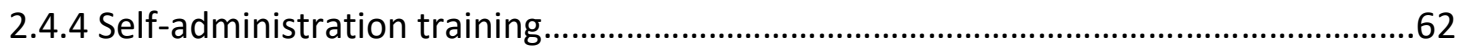


2.4.4.1 Cocaine-prime drug reinstatement tests

2.4.4.2 Establishing a dose-response cocaine self-administration model.......................65

2.4.4.3 Dose-response cocaine self-administration tests..............................................65

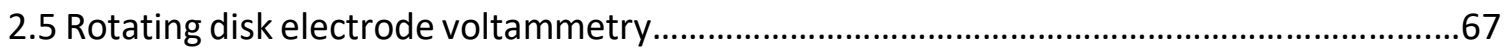

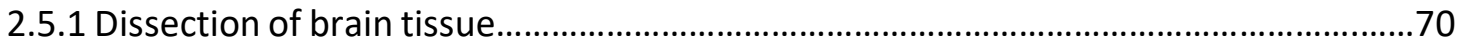

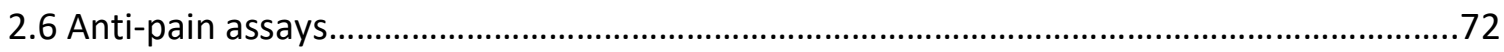

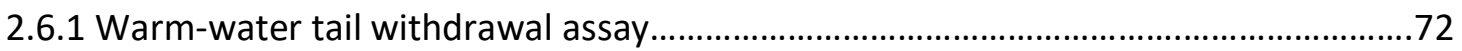

2.6.1.1 Time-course tail withdrawal effects...................................................................72

2.6.1.2 Cumulative dose-response tail withdrawal effects.........................................73

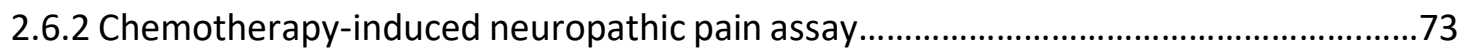

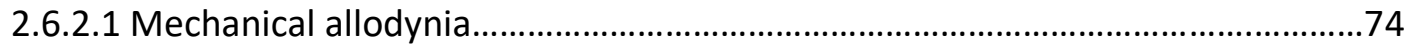

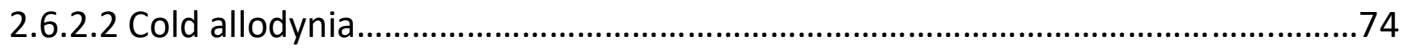

2.6.2.3 Evaluation of acute MP1104 administration on chemotherapy-induced neuro-

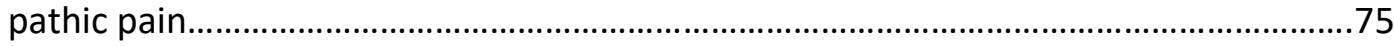

2.6.2.4 Evaluation of chronic MP1104 administration on chemotherapy-induced neuro-

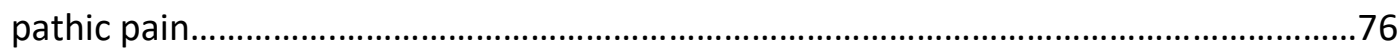

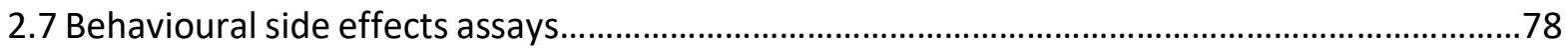

2.7.1 Spontaneous locomotor activity test.......................................................................

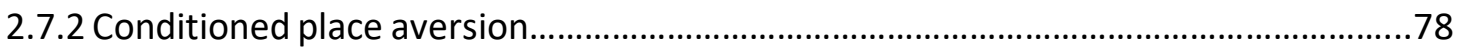

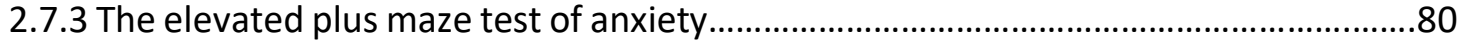

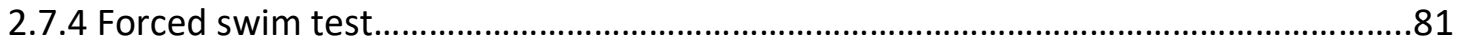

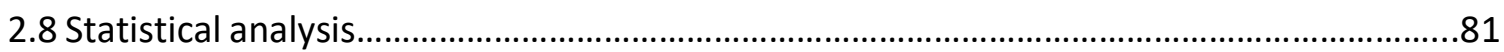

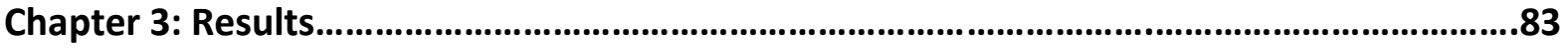

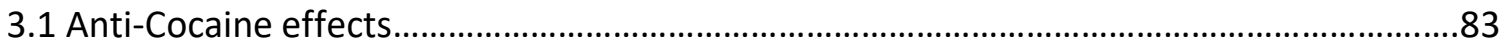

3.1.1 Effect of MP1104 on cocaine-induced drug seeking..................................................83

3.1.2 Effect of MP1104 on cocaine dose responding in rats trained to self-administer cocaine

3.1.3 Effect of MP1104 on dopamine transporter function...................................................89

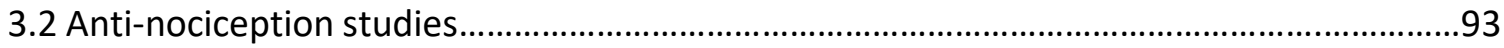


3.2.2 Cumulative dose-response effects using the warm-water tail withdrawal assay.

3.2.3 Cumulative dose-response effects of MP1104 on mechanical and cold allodynia.

3.2.4 Effect on chronic administration of MP1104 agonist on CINP-induced mechanical and cold allodynia. 101

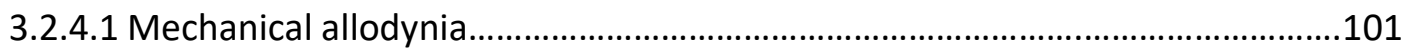

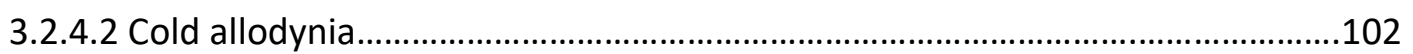

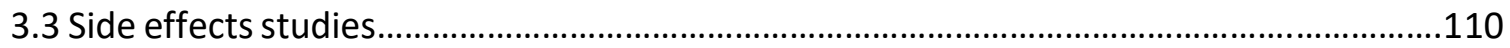

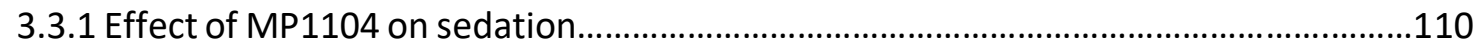

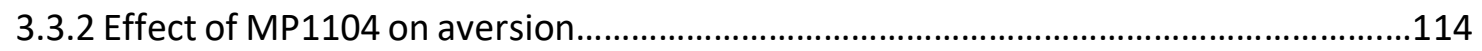

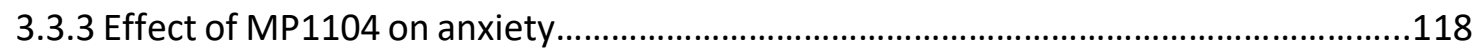

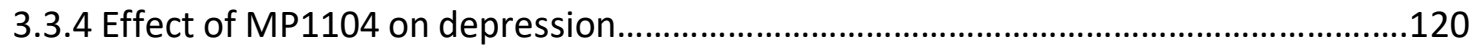

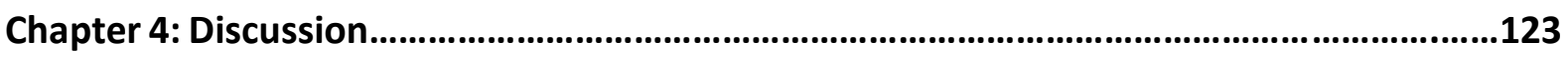

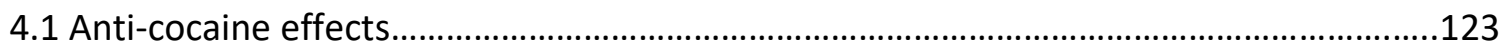

4.1.1 Effect of MP1104 on cocaine-induced drug seeking and drug taking........................123

4.1.1.1 MP1104 effect on cocaine taking behaviour.................................................127

4.1.2 Effect of MP1104 on dopamine transporter function..................................................129

4.1.2.1 MP1104 effects on DAT function in dStr and NAc brain regions.......................129

4.1.2.2 DA uptake by DAT in dStr and NAc brain regions............................................131

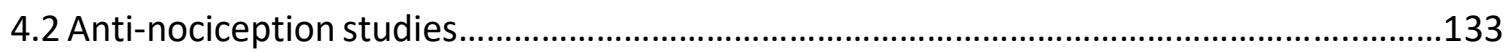

4.2.1 Effects of MP1104 in warm-water tail withdrawal assay..........................................133

4.2.2 Cumulative dose-response effects of MP1104 on mechanical and cold

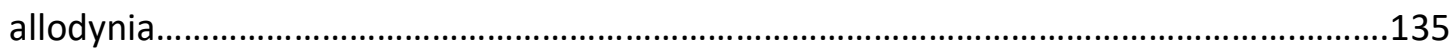

4.2.3 Effect on chronic administration of MP1104 agonist on mechanical and cold

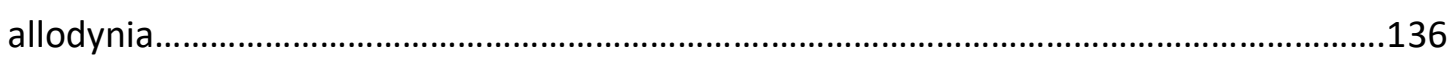

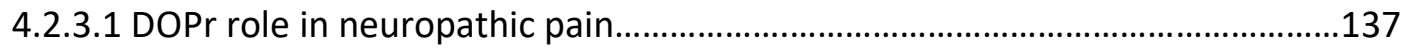

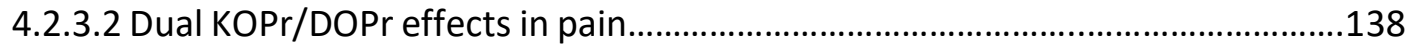




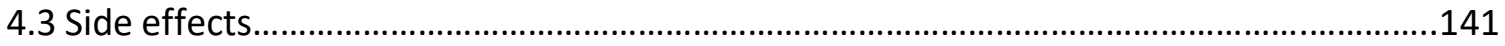

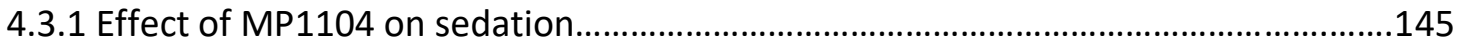

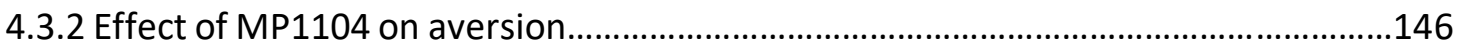

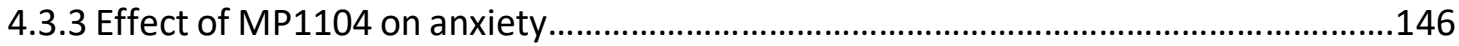

4.3.4 Effect of MP1104 on pro-depression........................................................................

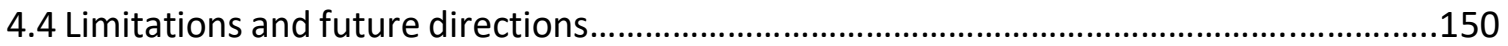

4.4.1 KOPr co-administration prevents morphine-induced tolerance................................154

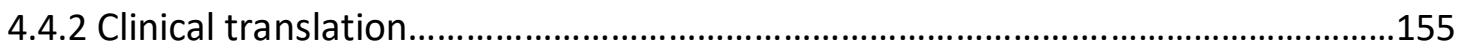

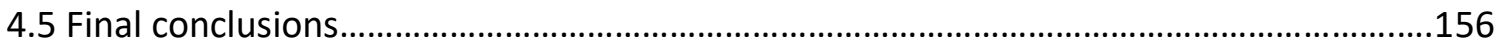

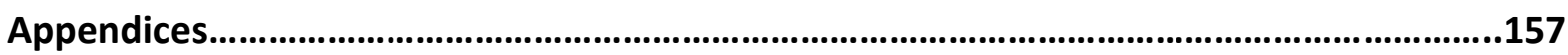

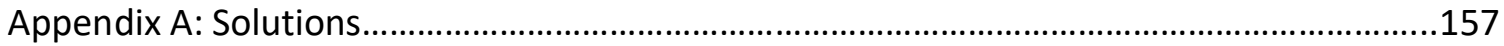

Appendix B: Determination of differences between drug naïve and U50,488 agonist pre-

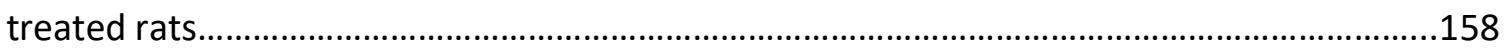

Appendix C: Latin square treatment order for drug self-administration study........................160

C.1 Cocaine reinstatement treatments.............................................................................160

C.2 Dose-response cocaine self-administration test treatments.....................................161

Appendix D: Warm-water tail withdrawal dose-response experiment....................................162

Appendix E: Testing scheme of the simplified up-down von Frey filament..............................164

Appendix F: Acute dose-response controls in the paclitaxel-induced neuropathic pain

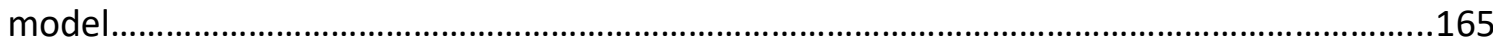

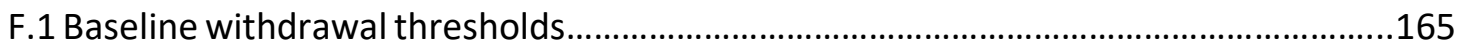

F.2 Day 15 pre-treatment withdrawal thresholds..............................................................166

F.3 Vehicle treatment in the dose-response method............................................................167 Appendix G: Chronic administration procedure controls in the paclitaxel-induced

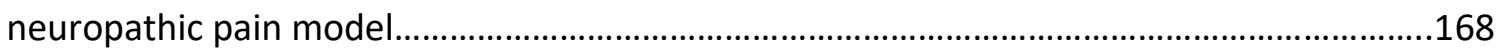

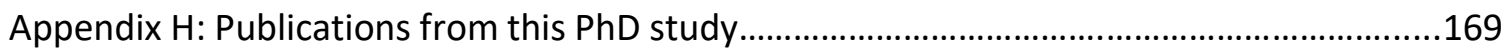

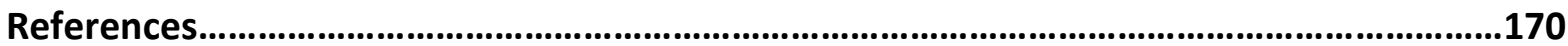




\section{List of Figures}

Figure 1.1: Kappa, mu and delta opioid receptors expression in the human brain, circuitry that modulates the reward pathway and kappa opioid receptor-dopamine transporter co-expression in the brain

Figure 1.2: An illustration of the three-stage addiction cycle ......................................................19

Figure 1.3: Kappa opioid receptor mediated signalling pathways.................................................26

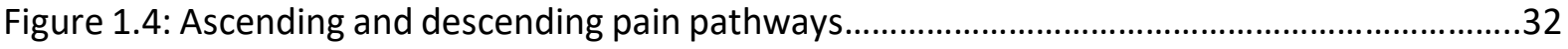

Figure 1.5: Regions of brain and spinal cord involved in pain processing compared to kappa, mu

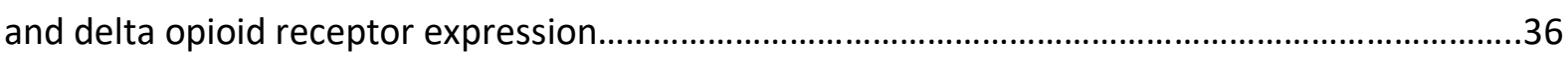

Figure 1.6: Chemical structures of MP1104 and 3-lodobenzoyl naltrexamine.................................52

Figure 2.1: Intrajugular catheter schematic diagram...................................................................60

Figure 2.2: Cocaine-induced reinstatement of drug seeking procedure schematic diagram...........64

Figure 2.3: Dose-response cocaine self-administration procedure schematic diagram..................66

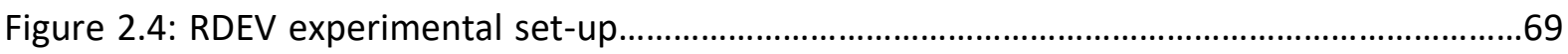

Figure 2.5: Interaural coordinates for rat brain dissection.................................................................70

Figure 2.6: Schematic diagram of experimental design for acute dose-response procedure in

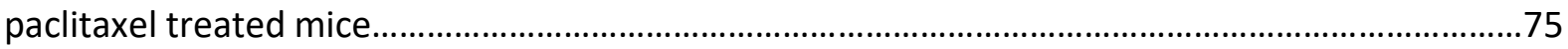

Figure 2.7: Schematic diagram of experimental design for chronic treatment of MP1104 or morphine in mice with established paclitaxel-induced neuropathic pain .........................................77

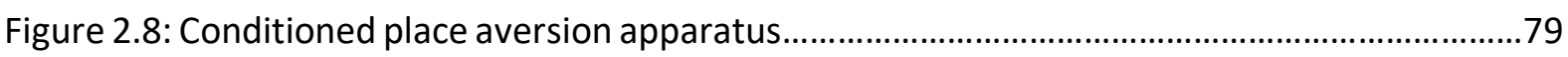

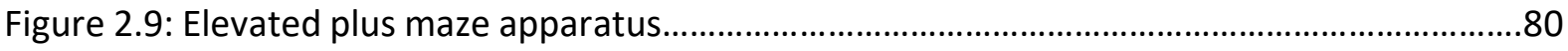

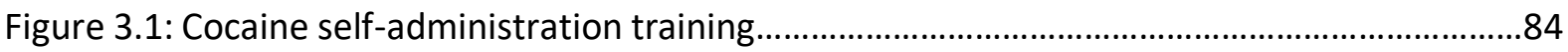

Figure 3.2: MP1104 attenuates cocaine-primed reinstatement of cocaine seeking in rats.............86

Figure 3.3: MP1104 shifts cocaine dose-response self-administration curve downwards...............88

Figure 3.4: MP1104 showed increase of DA uptake in rat dStr and NAc tissues................................90

Figure 3.5: Duration of action of MP1104 in warm-water tail withdrawal assay in rats...................94

Figure 3.6: Dose-response effects of MP1104 in rats and mice using warm-water tail withdrawal assay.

Figure 3.7: Dose-response effects of MP1104 and morphine in mice with established paclitaxelinduced neuropathic pain. 100

Figure 3.8: Treatment of paclitaxel-induced mechanical and cold allodynia...... 104 
Figure 3.9: Mechanical and cold allodynia with measurements before daily treatment. 108

Figure 3.10: Effects of MP1104 on sedation..............................................................................112

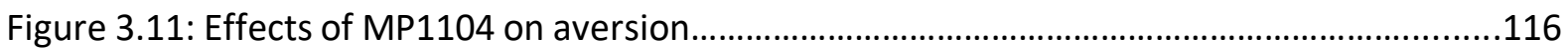

Figure 3.12: Effects of MP1104 on anxiety ..................................................................................119

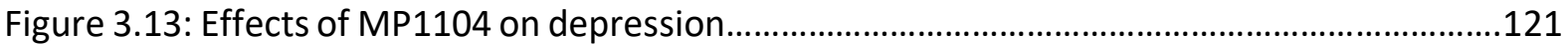

Figure B.1: KOPr pre-treatment effects in cocaine self-administration acquisition time in rats

Figure D.1: Vehicle control group in the warm-water tail withdrawal dose-response assay.........163

Figure E: Examples of the simplified up-down von Frey filament testing scheme.

Figure F.1: Day 0 pre-paclitaxel mechanical and cold threshold values showed no differences between the assigned dose-response treatment groups.

Figure F.2: Day 15 post-paclitaxel mechanical and cold allodynia values showed no differences between the assigned dose-response treatment groups.

Figure F.3: In mice with established paclitaxel-induced neuropathic pain vehicle treatment did not have an anti-nociceptive effect. 167

Figure G.1: Mechanical withdrawal thresholds and cold response times on day 15 before assignment of treatment groups 


\section{List of Tables}

Table 1.1: Acute effects of kappa opioid receptor agonists on anti-cocaine behavioural

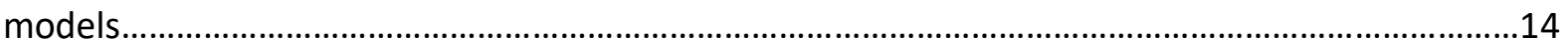

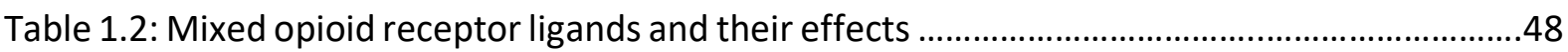

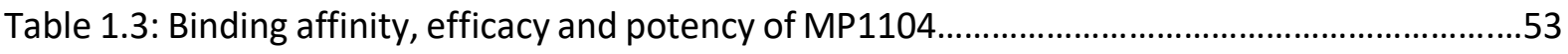

Table 2.1: Dissected brain regions, their coordinates and weights...................................................70

Table 3.1: Dose-response effects of MP1104 differences in rats and mice.......................................98

Table 4.1: Acute effects of KOPr agonists on KOPr-mediated adverse effects...............................142

Table 4.2: Summary of anti-cocaine, analgesic, and side effects produced by MP1104 in rats and

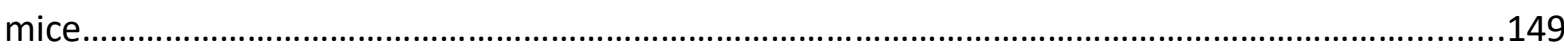

Table C.1: Treatment design used in cocaine-induced reinstatement of drug seeking.................160

Table C.2: Treatment design used in dose-response cocaine self-administration test...................161 


\section{Abbreviations}

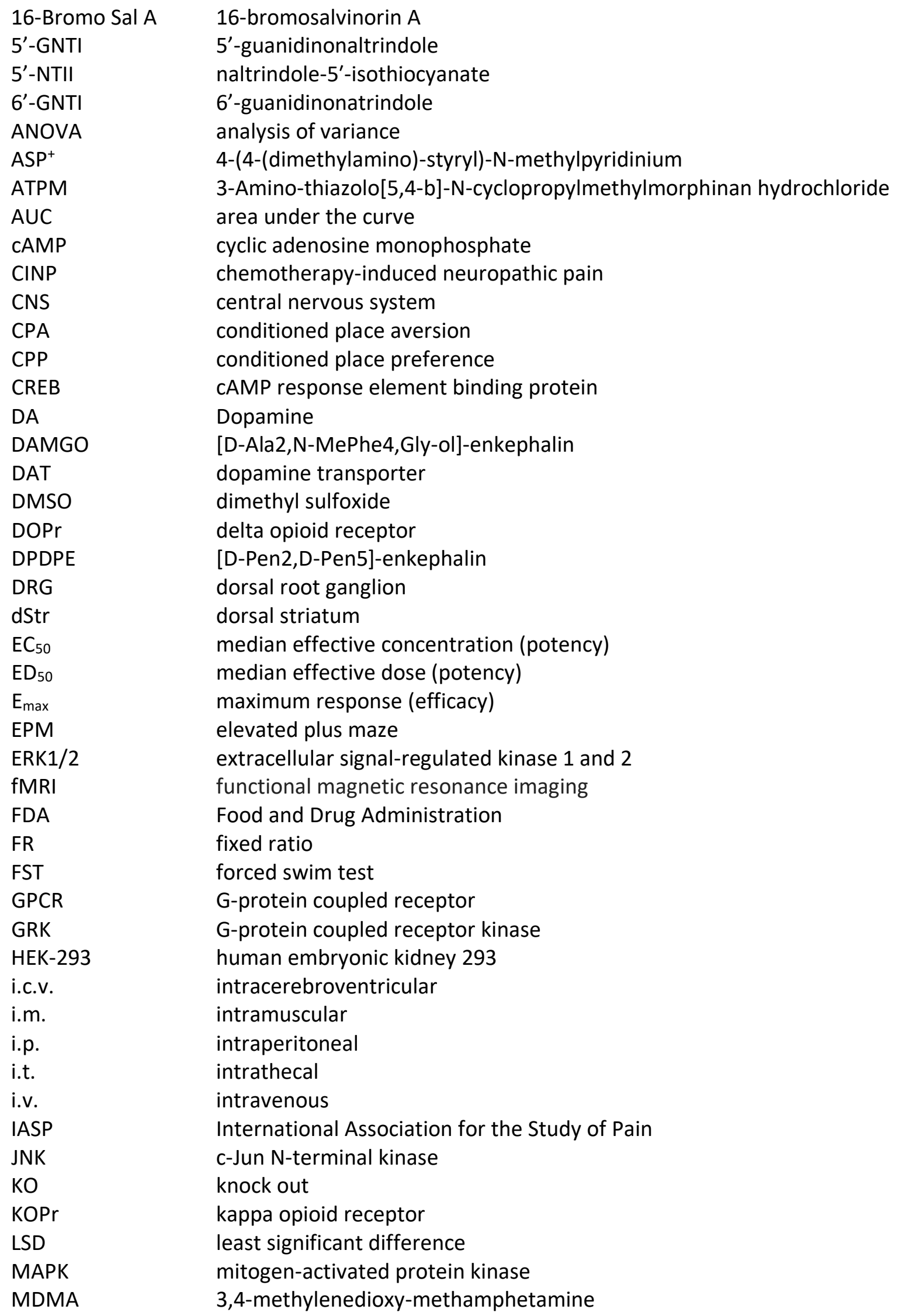




\begin{tabular}{|c|c|}
\hline Mesyl Sal B & mesyl Salvinorin B \\
\hline MOPr & mu opioid receptor \\
\hline MP1104 & $\begin{array}{l}\text { 17-Cyclopropylmethyl-3-hydroxy-4,5 } \alpha \text {-epoxy-7,8-en-6- } \beta-\left[\left(3^{\prime}-\right.\right. \\
\text { iodo)benzamido]-morphinan }\end{array}$ \\
\hline Mr2033 & $\begin{array}{l}\text { (+/-)-(1-R/S,5-R/S,2 = R/S)-5,9-dimethyl-2'-hydroxy-2-tetrahydrofurfuryl-6,7- } \\
\text { benzomorphan }\end{array}$ \\
\hline NAc & nucleus accumbens \\
\hline NET & norepinephrine transporter \\
\hline NOPr & nociception receptor \\
\hline nor-BNI & nor-binaltorphimine \\
\hline NTI & naltrindole \\
\hline p.o. & oral administration \\
\hline $\mathrm{pFC}$ & prefrontal cortex \\
\hline $\mathrm{PI} 3 \mathrm{~K}$ & phosphatidylinositol 3-kinase \\
\hline PKC & protein kinase $\mathrm{C}$ \\
\hline PNS & peripheral nervous system \\
\hline RB-64 & 22-thiocyanatosalvinorin A \\
\hline RDEV & rotating disk electrode voltammetry \\
\hline s.c. & subcutaneous \\
\hline Sal A & salvinorin $A$ \\
\hline SEM & standard error of the mean \\
\hline SERT & serotonin transporter \\
\hline SNC80 & $\begin{array}{l}\text { 4-(alpha-(4-Allyl-2,5-dimethyl-1-piperazinyl)-3-methoxybenzyl)-N,N- } \\
\text { diethylbenzamide }\end{array}$ \\
\hline SN & substantia nigra \\
\hline T.O & time out \\
\hline U50,488 & trans-3,4-dichloro- $N$-methyl- $N$-(2-1-pyrrolidinyl)-cyclohexyl-benzeacetamide \\
\hline U69,593 & 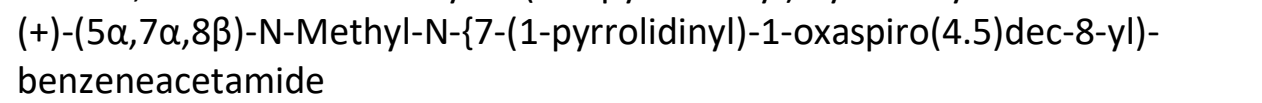 \\
\hline VTA & ventral tegmental area \\
\hline$\beta-F N A$ & $\beta$-funaltrexamine \\
\hline
\end{tabular}




\section{Chapter 1: Introduction}

\subsection{Background}

Addiction to drugs is a compulsive disorder of the brain that results in long-lasting changes in brain neurocircuitry and neurochemistry (Koob and Le Moal, 2008). In spite of extensive research by experts in the addiction field, there are currently no successful Food and Drug Administration (FDA) approved pharmacotherapies for psychostimulant abuse. Adding to this problem is the worldwide growing opioid crisis. Therefore, the development of non-addictive pain therapies and development of effective treatment for drug abuse are urgently needed. Kappa opioid receptor (KOPr) agonists have previously shown anti-addiction and cocaine antagonistic properties, effects attributed to their modulation of dopamine (DA) levels in the brain. KOPr agonists have also shown anti-nociceptive effects and are promising analgesic medications due to their low abuse potential, unlike clinically available $\mathrm{Mu}$ opioid receptor (MOPr) agonists. Unfortunately, traditional KOPr agonists present side effects such as sedation, aversion, anxiety, and depression that have prevented their translation into clinical use. Mixed opioid receptor agonists have been identified as potential therapeutic targets due to reduced side effects compared to selective KOPr, MOPr or delta opioid receptor (DOPr) agonists. Mixed opioid receptor agonists with improved profile show huge promise for the development of anti-addiction and analgesic pharmacotherapies. This $\mathrm{PhD}$ thesis explores the anti-addiction effects, anti-nociceptive properties and the side effect profile of a novel synthetic mixed opioid receptor acting agonist with an aim to assist in the development of a mixed opioid receptor agonist that may be useful as a non-addictive pain medication and as an effective treatment for psychostimulant abuse.

\subsection{Drug addiction}

\subsubsection{Definition and socioeconomic cost}

Drug addiction is a chronic, relapsing disorder. It is accompanied by the compulsion to seek and take the drug, loss of control in limiting drug intake and developing a negative emotional state (dysphoria, anxiety) during withdrawal (Koob and Volkow, 2010; Koob and Le Moal, 1997). Addicted individuals show intense drug craving and experience a high risk of relapse even after a 
long period of abstinence (Nestler, 2001). According to the recent survey conducted by United Nations Office on Drug and Crimes (UNODC) it has been estimated that worldwide about 275 million people (or around 5.6\% of the global population) aged between 15-64 years used drugs at least once during the year 2016 and about 31 million of people who use drugs suffer from drug use disorders (UNODC, 2018). The readily available access to drugs of abuse is a major concern, as studies carried out on American and Australian populations indicate that around one in five people who use illicit drugs regularly will develop drug dependence (Grant, 1996; Hall et al., 1999).

It has been estimated that in 2015 out of 450,000 deaths worldwide as a result of drug use, 167,750 deaths were associated with drug use disorders, and $76 \%$ of deaths from drug use disorders were related to the use of opioids (UNODC, 2018). Globally, alcohol (98.8\%), tobacco (61.3\%) and cannabis (57.6\%) use are highly prevalent (Global Drug Survey, 2018). Psychostimulants such as 3,4-methylenedioxy-methamphetamine (MDMA, known as 'ecstasy') (30.5\%), cocaine $(25.4 \%)$, and amphetamines $(21.3 \%)$ are also among the most popular drugs used (Global Drug Survey, 2018). In New Zealand, approximately $17 \%$ of people over the age of 16 years admit to recreational use of illicit drugs (Mason et al., 2010), with 83\% of regular opioid users, $55 \%$ of regular methamphetamine users, and $10 \%$ of regular MDMA users being classified as dependent upon their drug of abuse (Wilkins et al., 2006). The 2016 New Zealand Drug Harm Index estimated the social cost of drug-related harm and intervention costs in $2014 / 15$ as $\$ 1.8$ billion NZD (McFadden and Consultancy, 2016). The resultant increase in crime, increased healthcare costs, unemployment, and loss of productivity are estimated to cost $\$ 181$ billion in the USA (National Institute on Drug Abuse, 2008) and \$6 billion in New Zealand (Stack et al., 2009) each year, making treatments for addiction highly sought after.

Despite decades of effort focussed on the development of anti-addiction pharmacotherapies, there are currently no FDA approved drugs to treat psychostimulant addiction. In addition to the economic and social burden resulting from psychostimulant abuse (Grant et al., 2017), addiction to opioids has reached epidemic proportions (Rudd, 2016). Gold standard treatments for chronic pain include prescription opioids such as morphine, fentanyl and codeine (Toblin et al., 2011). These medications act by binding to the MOPr. Unfortunately, adverse side effects include respiratory depression, tolerance to analgesic effects, dependence, and constipation (Chu et al., 2006; Compton and Volkow, 2006; Goodman et al., 2007; Shook et al., 1990). In the USA, the rapid increase in the use of prescription and non-prescription drugs in recent years has led to the 'opioid 
crisis'. This has now become a worldwide concern due to the escalating number of deaths globally. Overdose can be lethal due to the effects on respiratory depression, and it has been reported opioid overdoses are the major cause of accidental deaths in the USA, killing more people than road accidents (Okie, 2010). It has been estimated that in 2017 out of 70,237 drug overdose deaths in the USA alone, $47,600(67.8 \%)$ deaths were related to the use of opioids (including prescription and synthetic opioids and heroin) (Hedegaard et al., 2018). In New Zealand, 10 deaths per year related to opioid or sedative use has been reported by the New Zealand Drug Harm Index for the year 2016, with the daily doses of prescription of opioids being quadrupled in the 10 year period from 2001-2011 (Berterame et al., 2016). Given the escalating addiction burden, there is even greater need for the development of non-addictive analgesics and effective anti-addiction pharmacotherapies.

\subsubsection{Neurophysiology of drug addiction and abuse}

\subsubsection{The natural reward pathway}

Addiction is intrinsically associated with the mesolimbic, mesocortical and nigrostriatal dopaminergic pathways of the brain, collectively referred to as the natural reward pathway (see Figure 1.1). The mesolimbic and the mesocortical pathways are generally referred to as the mesocorticolimbic pathways and are responsible for hedonic effects (mesolimbic) and motivation (mesocortical) (Wise, 2009). The nigrostriatal pathway is part of the basal ganglia loop and is functionally involved in motor control (Wise, 2009). It is important to note that the nigrostriatal pathway has also been shown to be involved in behavioural flexibility (Cools et al., 2001; Grospe et al., 2018), and in habit formation/learning (Faure et al., 2005; Mura and Feldon, 2003; SeipCammack et al., 2017). Moreover, studies reported that the nigrostriatal circuitry has been shown to be necessary for the development of cocaine seeking habits (Belin et al., 2009; Yin et al., 2008), and is particularly important during and after the shift from recreational to compulsive drug use (Dayan and Balleine, 2002; Everitt and Robbins, 2005; Volkow et al., 2006). The mesocorticolimbic reward pathway is also implicated as being an important link in the neurocircuitry of reward (Fibiger and Phillips, 1988; Wise, 2009), regulating motivation and reinforcement of behaviours that are advantageous to the survival of a species such as eating, drinking, and sex. The Ventral tegmental area (VTA), the nucleus accumbens (NAc) and the pre-fontal cortex (pFC) are the three main areas of brain associated in the generation of reward via the mesocorticolimbic pathway 
(Phillips and Fibiger, 1978). However, recent findings suggest the lateral habenula also plays a major role in processing reward (Lecca et al., 2014; Velasquez et al., 2014).

\subsubsection{Role of dopamine in regulating drug addiction}

DA is the neurotransmitter that plays a major role in activating the neurobiological changes associated with addiction (Volkow et al., 2007). DA is synthesised within the VTA by dopaminergic neurons and extends to the NAc and pFC forming the mesolimbic and mesocortical neural pathways respectively (Swanson, 1982) (see Figure 1.1). VTA stimulation results in the release of DA at the synaptic terminal in the NAc and pFC producing a euphoric or pleasurable feeling in response to behavioural stimuli such as food and drink ingestion, and sexual activity (Pfaus et al., 1990; Yoshida et al., 1992). The physiological actions of DA on post-synaptic target cells in the NAc and pFC are initiated by five types of G-protein coupled receptors (GPCR), D1 to D5 (Missale et al., 1998). This family of GPCR for DA are classified as either D1-like (D1 \& D5) or D2-like (D2, D3 \& D4) receptors according to their respective stimulatory or inhibitory interactions with adenylyl cyclase (Kebabian, 1978; Missale et al., 1998). DA action is ceased by the dopamine transporter (DAT), which transports DA back into pre-synaptic neurons, where DA is either recycled or degraded by monoamine oxidase or catechol-O-methyltransferase (Elsworth and Roth, 1997).

DAT is expressed in axons, cell soma and dendrites on the extra-synaptic plasma membrane. DAT regulates its action through ubiquitination, membrane internalisation or insertion, phosphorylation, protein-protein interactions and glycosylation (Chen et al., 2010; Eriksen et al., 2010; Li et al., 2004; Torres, 2006). DAT was also found to be selectively expressed in neurons located in the VTA (Ciliax et al., 1995), and DAT protein was found in very high amounts in the NAc and dorsal striatum (dStr) regions of the rodent brain where it is involved in reward (Boja and Kuhar, 1989; Scheffel et al., 1991). DA levels in the synapse are regulated by activation of DAT on the presynaptic neuron and DAT functions as the primary mechanism by which DA is cleared from the synapse (Torres, 2006). However, studies have found that DA uptake may also occur via the norepinephrine transporter (NET) (Carboni et al., 1990) and serotonin transporter (SERT) (Larsen et al., 2011). SERT has been shown to have weak affinity to take up DA (Hoffman et al., 1991), whereas NET was thought to transport DA (Horn, 1973; Raiteri et al., 1977), especially in brain regions that have a minimum amount of DAT. For example, in the medial pFC, which expresses 
large amount of NET (Mazei et al., 2002; Morón et al., 2002) and minimal amount of DAT (Freed et al., 1995; Sesack et al., 1998), which is thought to be involved with the majority of DA uptake in this brain region.

All known drugs of abuse cause hyperactivity of the natural dopaminergic reward pathway, by increasing extracellular levels of DA (Di Chiara and Imperato, 1988a). The exact mechanism through which drugs of abuse achieve this may differ.

The 'anhedonia hypothesis' proposes that brain dopaminergic systems mediate the pleasure produced by food and other unconditioned incentives such as sex or drugs of abuse. After the administration of DA antagonists, according to the 'anhedonia hypothesis', "all of life's pleasures - the pleasures of primary reinforcement and the pleasures of their associated stimulilose their ability to arouse the animal" (Wise, 1982, 1985). The 'anhedonia hypothesis' suggests that DA in the brain plays a very important role in the motivation associated with positive rewards such as food, water, psychostimulants and opioids (Wise, 2008). Hence, modulating the effects of DA is a way of curbing the reinforcing effects of addictive drugs.

In support, several studies reported that natural rewards such as food and sex, and most substances (alcohol, amphetamine, caffeine, cocaine, marijuana, nicotine, opiates, and phencyclidine) that are abused by humans, have shown to increase extracellular concentrations of mesolimbic DA (Carlezon and Wise, 1996; Di Chiara, 1998; Gardner, 1997; Jentsch and Taylor, 1999). Stimulants like cocaine and amphetamine directly amplify the mesolimbic dopaminergic signal at the postsynaptic DA receptor through different synaptic mechanisms. The amphetamines increase synaptic DA primarily by increasing DA release from the synaptic vesicles (Bunney and Aghajanian, 1978). Amphetamine action requires both DAT and the vesicular monoamine transporter (VMAT2) (Pifl et al., 1995; Jones et al., 1998). Upon entering the presynaptic neuron, amphetamine causes reversal of DAT through DAT phosphorylation (Miller, 2011). The DAT is responsible for reabsorbing synaptic DA back into the presynaptic neuron, and occupancy of the DAT by cocaine prevents DA re-uptake (Adinoff, 2004). Due to their direct effect upon dopaminergic activity, the stimulants (particularly cocaine) are considered prototypic drugs of reward and have thus become the focus of biological and treatment studies (Adinoff, 2004). The availability of DA in the brain is modulated by the DAT, a plasma membrane protein that actively trans-locates released transmitter from the extracellular space into the presynaptic neuron. This DAT controls the DA neurotransmission by driving reuptake of extracellular transmitter into presynaptic neurons (Vaughan and Foster, 2013). 
Although the 'anhedonia hypothesis' is widely accepted, there are studies that highlight that opioid receptor activity is much more important in mediating hedonic reactions than the DA systems. Berridge and Robinson (1998) suggested that DA may be more important to incentive salience attributions and necessary for 'wanting' (motivation to seek drug) incentives, but not for 'liking' (hedonic effects of the drug) them. Many studies have reported that hedonic effects are mediated by opioidergic mechanisms in both humans (Buchel et al., 2018) and animals (Ismail et al., 2009; Le Merrer et al., 2009; Pecina and Berridge, 2005; Smith et al., 2011). For example, Pecina and Berridge (2005) demonstrated that the shell of the NAc contains a hedonic hotspot in which the stimulation of MOPrs increases the "liking" for food reward, as measured by the amplification of positive affective orofacial reactions to sucrose in rats, and the "wanting" for food as reflected by increased eating behaviour. In humans a within-subject, cross-over, pharmacological challenge study with the opioid receptor antagonist naloxone in combination with functional magnetic resonance imaging ( $\mathrm{fMRI}$ ) was conducted to investigate the role of the opioidergic system in processing the pleasure of rewards (erotic stimuli) (Buchel et al., 2018). The study observed that naloxone reduced pleasure rating to erotic stimuli in male volunteers $(n=21)$ and further fMRI neuroimaging revealed that the opioid receptor antagonist led to a reduction of neuronal activation in the mesocorticolimbic system, showing that the opioidergic system is involved in the hedonic processing of erotic stimuli (Buchel et al., 2018). 


\subsubsection{Kappa opioid receptor role in drug addiction}

\subsubsection{Regulation of the reward pathway by the kappa opioid receptor system}

Activation of the endogenous KOPr ligand, dynorphin, is believed to function to oppose positive reinforcement (Shippenberg et al., 2007). Upregulation of KOPr is also believed to be responsible for exerting negative effects during drug withdrawal (Koob and Volkow, 2010). For example, modulation of the KOPr system, via both agonists and antagonists, is believed to have potential in modulating different stages of addiction. KOPr antagonists such as nor-binaltorphimine (norBNI) and JDTic, have been shown to prevent behavioural adaptations related to stress (Beardsley et al., 2005; Jackson et al., 2013). Since there is a strong connection between stress and drugrelapse in humans (Koob, 2009), KOPr antagonists are being explored as potential pharmacotherapies for preventing stress-induced relapse (Beardsley et al., 2005; Bruchas et al., 2010; Redila and Chavkin, 2008). On the other hand, traditional KOPr agonists like trans-3,4-

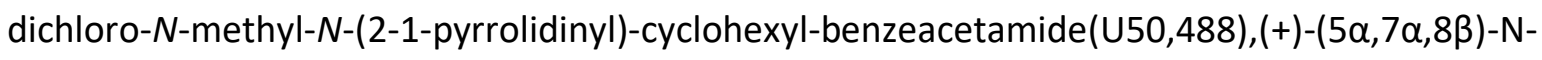
Methyl-N-\{7-(1-pyrrolidinyl)-1-oxaspiro(4.5)dec-8-yl)-benzeneacetamide $(U 69,593)$, spiradoline and a neoclerodane diterpene compound Salvinorin A (Sal A) have shown to possess anti-cocaine properties in preclinical models of drug use and are reported to attenuate the rewarding effects of cocaine (see Table 1.1). Few KOPr agonists, possessing anti-addictive properties are being explored as possible formulations to reduce the abuse potential of MOPr pain medications (Freeman et al., 2014; Naylor et al., 2015). Also, KOPr agonists reduce tolerance to morphine analgesia. A few studies have shown that co-administration of traditional KOPr agonist U50,488 reduced tolerance in morphine tolerant animals (discussed in detail in section 4.4.1).

As per the 'anhedonia hypothesis' (discussed in section 1.2.2.2), the reinforcing effects of drugs of abuse can be alleviated via the modulation of DA levels (Wise, 2008). Activation of KOPr has been shown to produce reduced DA neurotransmission (Di Chiara and Imperato, 1988b; Wee and Koob, 2010). In fact, the mechanisms through which KOPr exerts its anti-addiction properties are via reducing basal DA release (Carlezon et al., 2006; Spanagel et al., 1992; Spangler et al., 1997; Wee and Koob, 2010), drug-evoked DA release (Zhang et al., 2004a, 2004b) and by increasing DA reuptake via DAT (Kivell et al., 2014b; Simonson et al., 2015). These studies suggest that the KOPr system can be targeted for the development of potential anti-addiction pharmacotherapies. 


\subsubsection{Regulation of dopamine transporter function by kappa opioid receptor}

KOPr agonists are reported to influence DAT function. In chronic cocaine users KOPr and DAT have been shown to be upregulated, with both systems acting synergistically to regulate DA levels (Kivell et al., 2004; Thompson et al., 2000). Svingos et al. (2001) showed that KOPr and DAT lie in close proximity at DA nerve terminals, suggesting a role of $\mathrm{KOPr}$ in regulating DAT and subsequently, DA levels. DAT is co-localised with KOPr in the dStr, amygdala, NAc, VTA, SN and pFC at the terminals and axons (Ciliax et al., 1999; Ciliax et al., 1995; Simonin et al., 1995) (see Figure 1.1). 


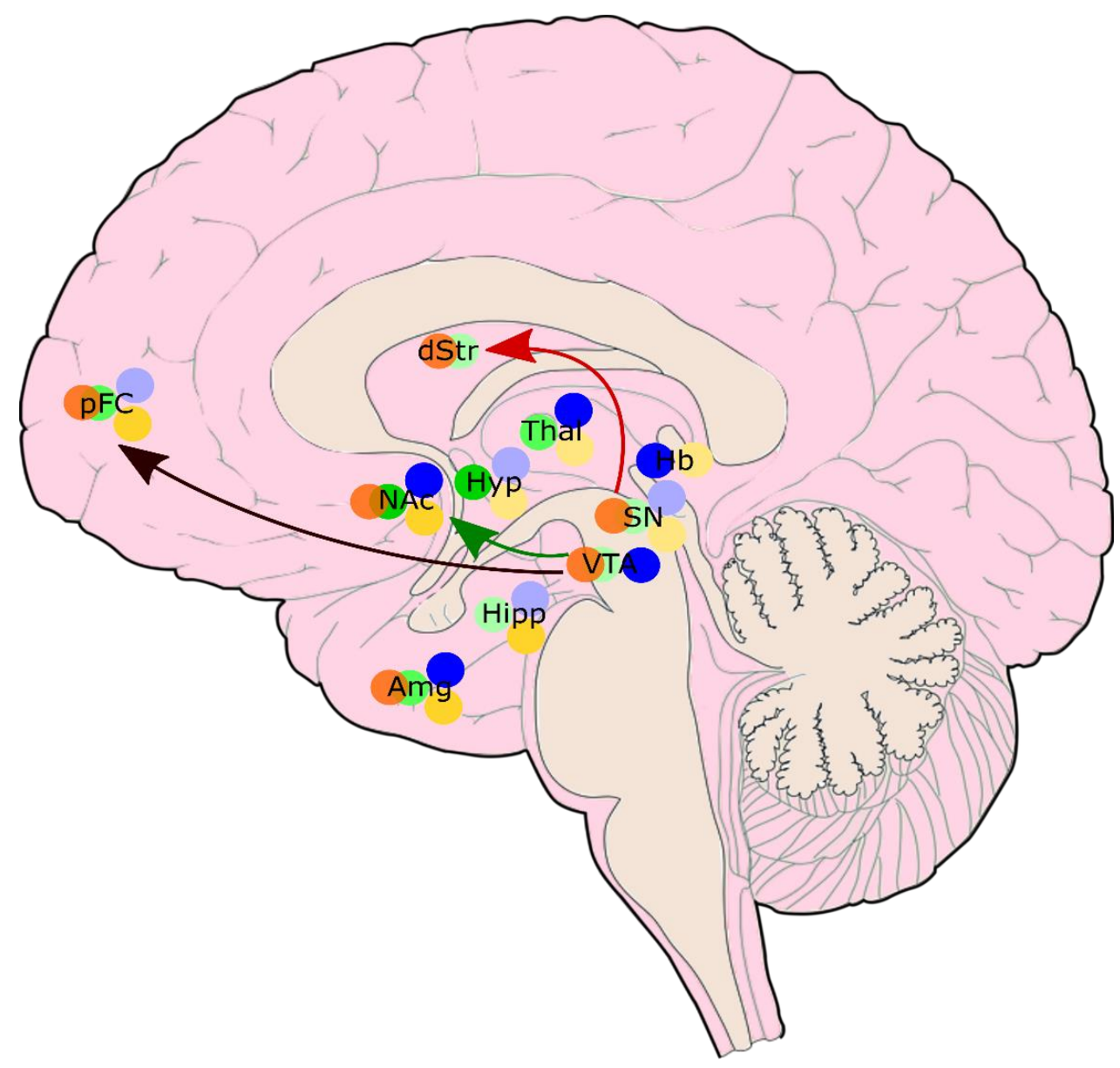

Figure 1.1: Kappa, mu and delta opioid receptors expression in the human brain, circuitry that modulates the reward pathway and kappa opioid receptor-dopamine transporter coexpression in the brain

Kappa opioid receptor (KOPr) (green circle), mu opioid receptor (MOPr) (blue circle) and delta opioid receptor (DOPr) (yellow circle) expression in relevant areas of reward and aversion are shown (darker colour indicates higher densities of KOPr, MOPr or DOPr expression). Dopaminergic neurons projecting from the VTA to the NAc make up the mesolimbic pathway (green arrow), whereas neurons projecting from the VTA to the $\mathrm{pFC}$ make up the mesocortical pathway (maroon arrow). The nigrostriatal pathway (red arrow) has dopaminergic neurons with cell bodies in the $\mathrm{SN}$ which project to the dStr. Co-expression of KOPr and dopamine transporter (DAT) (orange circle) is observed in areas of reward (VTA, NAc, SN and pFC) and also in the amygdala. Figure adapted from Darcq and Kieffer (2018) and Lutz and Kieffer (2013a). KOPr, MOPr and DOPr expression based on radio-ligand binding and quantitative real-time reverse transcriptase polymerase chain reaction data from Le Merrer et al. (2009) and Peng et al. (2012) respectively. Expression profiles of DAT and KOPr in human brain tissue, based upon tissue immunohistochemistry assays by Ciliax et al. $(1995,1999)$.

Amg, amygdala; dStr, dorsal striatum; Hb, habenula; Hipp, hippocampus; Hyp, hypothalamus; NAc, nucleus accumbens; pFC, pre frontal cortex; SN, substantia nigra; Thal, thalamus; VTA, ventral tegmental area. 
The interaction between KOPr activation and DAT has been identified as a potential target for the development of therapeutic drugs to treat addiction. In vitro studies revealed that KOPr expressing cells enhanced the cell-surface expression of DAT and increased DA uptake when the KOPr agonist Sal A was applied to cultured cells co-expressing DAT and KOPr (Kivell et al., 2014a). Similarly Mesyl Salvinorin B (Mesyl Sal B), an analogue of Sal A and the traditional KOPr agonist, U50,488 increased DAT function in vitro in rat NAc tissue using rotating disk electrode voltammetry (RDEV) (Simonson et al., 2015). In the same study, Simonson et al. (2015) showed that Sal A and U69,593, a traditional KOPr agonist, also increased DAT function in in vitro assays using human embryonic kidney (HEK)-293 cells. To further support this close association between KOPr and DAT, in studies evaluating co-expression of KOPr and DAT found the formation of KOPrDAT complexes in striatal synaptosomes and this KOPr and DAT interaction is enhanced by the administration of KOPr agonists (Kivell et al., 2014a). Several studies have also reported that administration of KOPr agonists and endogenous KOPr activation reduces DA release (Carlezon et al., 2006; Gray et al., 1999), and there are differences in the regulation of DAT in different brain regions including the NAc and dStr brain regions (Thompson et al., 2000) (discussed in detail in sections 4.1.2.2).

KOPr agonists have cocaine-opposing actions, which make them suitable to be used as anticocaine compounds, which act by blocking cocaine-induced alterations in the dopaminergic system via DAT regulation (Chefer et al., 2000). The mechanism of action by which cocaine shows its effects is by releasing DA, blocking DAT and preventing reuptake of DA into the pre-synaptic neuron, thereby resulting in raising the levels of DA in the synapse. Studies showed that cocaine mobilises DAT to the cell surface, in HEK-293 and neuro-2A mouse neuroblastoma cells but these effects were not seen in African green monkey fibroblast (COS7) cells (Daws et al., 2002; Zhang et al., 1998). However, a study by Heal et al. (2014) revealed that cocaine not only inhibits DA uptake, but also reverses the direction of DAT transport, termed as efflux.

In the dStr of male Sprague-Dawley rats, subcutaneous (s.c.) administration of U69,593 (0.32 $\mathrm{mg} / \mathrm{kg}$ ) and cocaine for five days showed no significant effects on DAT density, suggesting that alterations in DAT levels following U69,593 treatment directly counteracts those of cocaine (Collins et al., 2001b). Similarly, another study reported that intracranial perfusion of U69,593 (1 $\mu \mathrm{g} / \mathrm{side}$ ) into both sides of NAc prior to cocaine injections (20 mg/kg, intraperitoneal, i.p.) for 3 days also prevented alterations in DA neurotransmission that occur during abstinence in rats (Chefer et al., 1999). Furthermore, when KOPr agonists were administered prior to cocaine and cocaine-induced hyperactivity (Chartoff et al., 2008; Chefer et al., 2000) and locomotor 
sensitisation evaluated (Morani and al, 2012; Shippenberg et al., 1996), KOPr agonists attenuated cocaine-stimulated behavioural locomotor effects.

Acute KOPr treatment regulates extracellular DA concentrations. This effect is by decreasing DA release. For example, U50,488 $(0.5,2.5 \& 10 \mathrm{mg} / \mathrm{kg}$, s.c.) decreases DA levels in the NAc and from the dorsal caudate of freely moving rats (Di Chiara and Imperato, 1988b). Using quantitative microdialysis techniques, a single injection of $U 69,593(0.32 \mathrm{mg} / \mathrm{kg}$, i.p.) has been shown to increase DAT function in the rat NAc, and this effect is blocked by pre-treatment with the KOPr antagonist, nor-BNI (10 mg/kg, s.c.) (Thompson et al., 2000). Another study showed that acute administration of U50,488 (500 nM) and the KOPr agonist Mesyl Sal B (500 nM) increased DA clearance in male Sprague-Dawley rat dStr, NAc, and pFC brain tissue following a single addition of DA (2 $\mu \mathrm{M})$ using RDEV zero trans model (Simonson et al., 2015). This increase in DA clearance was inhibited by prior incubation of dStr tissue with nor-BNI (1 $\mu \mathrm{M}, 30 \mathrm{~min})$ indicating the decrease in DA release is KOPr mediated (Simonson et al., 2015). In contrast, repeated treatment with KOPr agonists have opposite effects on DA levels. For example, U69,593 $(0.32 \mathrm{mg} / \mathrm{kg}$, s.c.) administered daily to rats for five days caused a significant decrease in DAT function in the NAc (Thompson et al., 2000). A decrease in DAT density in the caudate putamen and NAc of rats was also seen using auto-radiographic techniques (Collins et al., 2001a \& b). This highlights the complexity of KOPr function in the brain and differences in acute vs chronic treatment, with repeated KOPr administration having the opposite effect to acute treatment.

Overall, these studies highlight the importance that interaction between KOPr activation and DA levels, making KOPr a potential target for the development of therapeutic drugs to treat addiction. Although these previous studies have utilised pure KOPr agonists, our study sought to determine whether the mixed actions of 17-Cyclopropylmethyl-3-hydroxy-4,5 $\alpha$-epoxy-7,8-en-6- $\beta-\left[\left(3^{\prime}-\right.\right.$ iodo)benzamido]-morphinan (MP1104) were sufficient to modulate DAT function in rat NAc and dStr using RDEV. This is crucial to ascertain the mechanism by which MP1104 mediates anticocaine behavioural responses in rats. 


\subsubsection{Anti-cocaine effects of traditional kappa opioid receptor agonists}

The traditional KOPr agonists include the arylacetamide derivatives class such as U50,488, U69,593, spiradoline, enadoline, PD117302 (a cyclohexyl benzeneacetamide derivative) and ICI 204,448. Cyclazocine, ethylketocyclazocine, (+/-)-(1-R/S,5-R/S,2 = R/S)-5,9-dimethyl-2'-hydroxy2-tetrahydrofurfuryl-6,7-benzomorphan (Mr2033) and bremazocine belong to benzomorphans class, whereas nalmefene, nalfurafine, and cyclorphan belong to morphinans. Terpenoids class includes collybolide (Gupta et al., 2016), 22-thiocyanatosalvinorin A (RB-64) (Yan et al., 2009), and Sal $A$ and its synthetic analogues.

Acute administration of KOPr agonists is well known to reduce cocaine self-administration in rats (Glick et al., 1998; Schenk and Partridge, 2001) and rhesus monkeys (Mello and Negus, 1998, 2000) (see Table 1.1), and attenuate morphine self-administration in both rats (Glick et al., 1995) and mice (Kuzmin et al., 1997). Administration of KOPr agonists is also known to decrease ethanol (Lindholm et al., 2001) and nicotine (Ismayilova and Shoaib, 2010) self-administration in rats. Taken together, these studies highlight the potential for utilising KOPr agonists to treat drug addiction. Moreover, due to their low abuse potential, KOPr agonists are also being investigated for development of non-addictive pain medications. Few KOPr agonists have been explored as potential formulations to reduce abuse potential of clinically available pain medications. Freeman et al. (2014) showed that co-infusion of selective KOPr agonist like Sal A with either cocaine or MOPr agonist remifentanil reversed the lever preference in rhesus monkeys. Similar effects were also seen using oxycodone alone or in a mixture with Sal A (Naylor et al., 2015). These studies demonstrate that Sal A can 'punish' the self-administration of MOPr agonists remifentanil or oxycodone and a psychostimulant cocaine, hence KOPr agonist can be delivered in combination formularies for prescription medications. KOPr agonists are therefore being investigated as abuse-deterrent formulations. Recently, Townsend et al. (2017) showed that intravenous (i.v.) coadministration of nalfurafine $(0.32,1$ or $3.2 \mu \mathrm{g} / \mathrm{kg} / \mathrm{inj}$, i.v. $)$ with MOPr agonist oxycodone reduced reinforcing effects of the oxycodone $(56 \mu \mathrm{g} / \mathrm{kg} / \mathrm{inj}$, i.v.) in a progressive ratio self-administration procedure in male Sprague-Dawley rats. Further measuring the anti-nociceptive effects using hot plate test showed oxycodone and nalfurafine each produced dose-dependent anti-nociception, and the mixtures produced additive anti-nociception without producing respiratory depression in rats (Townsend et al., 2017). These results suggest that nalfurafine may increase the thermal antinociceptive effects while reducing the reinforcing and respiratory-depressant effects of oxycodone. 
Although KOPr agonists hold promise as possible anti-addictive pharmacotherapies, their clinical applicability is limited due to a number of side effects exerted by them in both human and animal models (see Table 4.1 for acute effects of KOPr agonists on KOPr-mediated adverse effects in animals). In humans, enadoline ( $\geq 25 \mu \mathrm{g} / \mathrm{kg}$ ) showed side effects that included dizziness, fatigue and sensory distortions in healthy volunteers (Walsh et al., 2001). Another KOPr agonist, cyclazocine produced irritability, dysphoria, hallucinations and insomnia which caused the cessation of clinical trials of cyclazocine for opioid dependence (Glick et al., 1998). Mr2033 (1.9 $3.8 \mu \mathrm{g} / \mathrm{kg}$, i.v.), induced dysphoria and psychotomimesis in male healthy volunteers, however at higher doses adverse effects such as weakness, anxiety, sweating, dizziness was observed (Pfeiffer et al., 1986). Hence, there is a need to develop KOPr agonists with a reduced side effect profile. 
Table 1.1: Acute effects of kappa opioid receptor agonists on anti-cocaine behavioural model

\begin{tabular}{|c|c|c|c|c|c|}
\hline \multirow{2}{*}{$\begin{array}{l}\text { Behavioural models to } \\
\text { assess anti-cocaine } \\
\text { effect }\end{array}$} & \multicolumn{5}{|c|}{ KOPr agonist } \\
\hline & U50,488 & U69,593 & Spiradoline & Salvinorin A & Animals \\
\hline \multirow[t]{2}{*}{$\begin{array}{l}\text { Cocaine self- } \\
\text { administration }\end{array}$} & $\begin{array}{c}\downarrow^{(1)} \\
10, \text { i.p. }\end{array}$ & $\begin{array}{c}\downarrow^{(2)} \\
0.32, \text { s.c. }\end{array}$ & $\begin{array}{c}\downarrow^{(1)} \\
2.5-5, \text { i.p. }\end{array}$ & $\begin{array}{c}\text { n.e. }^{(3)} \\
0.3,2.0 \text {, i.p. }\end{array}$ & $\begin{array}{l}\text { Male }{ }^{(2,3)} \text { and female } \\
\text { (1) Sprague-Dawley } \\
\text { rats Male } \\
\text { rats }\end{array}$ \\
\hline & $\begin{array}{c}\downarrow^{(4)} \\
0.032-0.1, \text { i.v. }\end{array}$ & - & - & $\begin{array}{c}\downarrow^{(5)} \\
\text { Variable doses, i.v. }\end{array}$ & $\begin{array}{l}\text { Male }{ }^{(4,5)} \text { and female } \\
\text { (4) rhesus monkeys }\end{array}$ \\
\hline $\begin{array}{l}\text { Cocaine-primed } \\
\text { reinstatement of } \\
\text { cocaine seeking } \\
\end{array}$ & $\begin{array}{c}\downarrow^{(6)} \\
30, \text { i.p. }\end{array}$ & $\begin{array}{r}\downarrow^{(2,6)} \\
0.32 \text {, s.c. }\end{array}$ & $\begin{array}{l}\downarrow^{(1)} \\
1, \text { i.p. }\end{array}$ & $\begin{array}{r}\downarrow^{(6)} \\
0.3, \text { i.p. }\end{array}$ & $\begin{array}{l}\text { Male }{ }^{(2,6)} \text { and female } \\
\text { (1) Sprague-Dawley } \\
\text { rats }\end{array}$ \\
\hline \multirow{2}{*}{$\begin{array}{l}\text { Conditioned place } \\
\text { preference }\end{array}$} & $\begin{array}{c}\downarrow \text { and } \uparrow(7,8) \\
\text { 5, i.p. }\end{array}$ & - & - & - & Male C57BL/6 mice \\
\hline & $\begin{array}{c}\downarrow^{(9)} \\
\text { 5, s.c. }\end{array}$ & - & - & - & $\begin{array}{l}\text { Male Sprague-Dawley } \\
\text { rats }\end{array}$ \\
\hline $\begin{array}{l}\text { Behavioural } \\
\text { sensitisation }\end{array}$ & $\begin{array}{l}\text { n.e. }{ }^{(10)} \\
10, \text { i.p. }\end{array}$ & $\begin{array}{l}\text { n.e. }{ }^{(10)} \\
0.3 \text {, s.c. }\end{array}$ & - & $\begin{array}{l}\downarrow^{(11)} \\
0.3, \text { i.p. }\end{array}$ & $\begin{array}{l}\text { Male Wistar rats }{ }^{(10)} ; \\
\text { Sprague-Dawley rats } \\
(11)\end{array}$ \\
\hline Hyperactivity & $\begin{array}{l}\downarrow^{(9,10)} \\
5, \text { s.c. } \\
\text { 10, i.p. }\end{array}$ & $\begin{array}{l}\downarrow^{(12,10)} \\
0.32, \text { s.c. } \\
0.3, \text { s.c. }\end{array}$ & - & $\begin{array}{l}\downarrow^{(13)} \\
\text { 2, i.p.; n.e. } \\
\text { 0.3, i.p. }\end{array}$ & $\begin{array}{l}\text { Male Wistar rats }{ }^{(10)} ; \\
\text { Male Sprague-Dawley } \\
\text { rats }(9,12,13)\end{array}$ \\
\hline
\end{tabular}

$\downarrow=$ attenuation, $\uparrow=$ potentiation, $-=$ study not found, n.e. $=$ no effect. Unless otherwise stated, doses are given in $\mathrm{mg} / \mathrm{kg}$.
1) Glick et al. (1995)
4) Negus et al. (1997)
7) Ehrich et al. (2015)
10) Vanderschuren et al. (2000)
13) Chartoff et al. (2008)
2) Schenk et al. (1999)
5) Freeman et al. (2014)
8) McLaughlin et al. (2006)
11) Morani et al. (2012)
3) Ewald (2016)
6) Morani et al. (2009)
9) Crawford et al. (1995)
12) Collins et al. (2001a) 


\subsubsection{Theories of drug addiction}

\subsubsection{Opponent process theory}

Opponent process theory postulates that many affective states, pleasant or aversive, are automatically opposed by centrally mediated mechanisms that reduce the intensity of these states (Solomon and Corbit, 1974; Stein, 1964). It is hypothesised that positive reinforcers, such as drugs, engage positive hedonic processes that are opposed by negative hedonic processes. The positive hedonic processes are hypothesised to be simple and stable and to follow administration of the drug closely in time. In contrast, the negative hedonic processes are of longer latency, slow to build up strength, and slow to decay (Solomon and Corbit, 1974). Within this framework, the intense pleasure of the cocaine "rush" or "high" is presumed to reflect a positive hedonic process, and the negative mood state associated with the drug wearing off or abstinence following a binge is presumed to reflect the opponent negative hedonic process (Solomon, 1977; Solomon and Corbit, 1974).

An adaptation of this concept, namely the 'negative allostasis theory' has been proposed by Koob and Le Moal (1997). According to this theory, any process in the brain has an opposing system that acts to restore the system to its homeostatic state (Koob and Le Moal, 1997, 2001). Chronic drug exposure causes deviations from the homeostatic set point. This shift, termed 'allostasis', is where the system is regulated outside its normal homeostatic range leading to a negative affective state. It is believed that this negative state is crucial for the development of addiction (Koob and Le Moal, 2001). The addiction cycle is made up of three stages, a preoccupation or anticipation stage, a binge or intoxication stage followed by withdrawal or negative effect (Koob and Le Moal, 1997, 2001) (see Figure 1.2). The preoccupation/ anticipation stage is characterised by a continual desire to take a drug, leading to the development of compulsion or anxiety. Whereas in the binge/intoxication stage, higher doses of the drug are taken in order to satisfy the arousal seen in the preoccupation/anticipation stage. These two stages are operated by positive reinforcement and mediated by dopaminergic systems in the brain. The final withdrawal/negative effect stage develops after the drug has been taken up and the effects are worn off (Koob and Le Moal, 2001). This stage consists of aversive symptoms such as dysphoria, depression and anxiety when access to the drug is ceased due to a shift in the homeostasis of the brain. The kappa opioid system has been suggested to play a role in stress responses and stress increases dynorphin in brain regions related to stress responses (Wee and Koob, 2010). Dynorphin activation is hypothesised to contribute to the negative emotional states, for example, KOPr agonists have shown to produce aversive effects in animals (refer to section 1.4.2 and Table 4.1 for details) and 
humans (Pfeiffer et al., 1986). Hence, the upregulation of KOPr is believed to be responsible for exerting negative effects during drug withdrawal (Koob and Volkow, 2010) (see Figure 1.2).

Over time, the addiction cycle becomes more intense, leading to a state where the drug is taken primarily to remove the negative affective state. At this point, the drug is taken in spite of known adverse consequences to the user. Since this is a slow process to develop, it is equally slow to reverse again. This theory is supported by research that shows that long-term changes in the brain of chronic drug users. Previously D2-like DA receptor family have both been shown to modulate DA levels in the synapse by decreasing DA synthesis and release (O'Hara et al., 1996; Tang et al., 1994) and modulating DAT function and cell surface expression (Bolan et al., 2007; Zapata et al., 2007). Chronic administration of cocaine produced long term reduction of the DA D2 receptors in monkeys (Nader et al., 2006), and also, in mice there was a reduction of excitatory transmission in the NAc (Thomas et al., 2001). Studies also reported lower levels of D2 receptor expression in striatum (Martinez et al., 2004) and pFC regions of brain (Volkow et al., 1993) and also reduced glucose metabolism in the pFC brain region that persisted up to four months following abstinence in cocaine users (Volkow et al., 1992). These long-term changes in the brain are explained by processes that change gene transcription, resulting in neural plasticity (Nestler, 2001).

\subsubsection{Incentive sensitisation theory}

The incentive sensitisation theory investigates the biochemical basis of craving. Persistent craving continues even after long periods of abstinence and dissociating drug-craving/wanting from drugliking (Robinson and Berridge, 1993, 2008). This theory states that drug addiction is developed due to gradual neuroadaptations that occur as a result of repeated drug use. The dopaminergic system attributes 'incentive salience', which is a motivational attribute developed by association between the environmental stimuli and the hedonic effects to natural rewards such as food and sex i.e., behaviours necessary for survival. In normal situations, pleasure accompanies and is required to assign incentive salience (Robinson and Berridge, 1993). However, in vulnerable individuals, drugs of abuse hijack the function of cells within reward regions and directly trigger incentive salience, thereby altering the circuitry to hypersensitise the system. Over repeated drug-use, the system becomes hypersensitive over a period of time to drugs and drug-associated stimuli, transforming drug wanting to craving (Berridge and Robinson, 1995; Robinson and Berridge, 1993, 2001). Hence, to this effect, this theory dissociates the act of drug 'wanting' 
(motivation to seek the drug) from liking (hedonic impact of the drug). Though these two processes often occur simultaneously, it has been postulated that drug wanting can occur independently of neural systems that mediate drug liking, but only drug wanting is affected by sensitisation (Berridge and Robinson, 1995). Sensitisation of neural systems associated with incentive salience leads drug users to increasingly wanting the drug while liking it less. This explains the intensity at which addicts pursue drugs irrespective of adverse effects despite reduced hedonic effects. The environmental cues such as drug-related stimuli and odour also influence sensitisation and can be an effective trigger to sensitised behaviours, resulting in high rates of relapse in abstinent users (Robinson and Berridge, 2001).

Incentive salience 'wanting' is mostly connected to reward cues, making those cues attentiongrabbing and attractive (Anderson and Yantis, 2013; Hickey and Peelen, 2015). 'Wanting' is mediated largely by the mesocorticolimbic system involving midbrain DA projections to NAc and other parts of striatum. In addicts, mesolimbic circuits are hyper-responsive to drug cues, which may cause strong cue-triggered 'wanting' to take drugs, leading to relapse (Robinson and Berridge, 1993). However, the 'liking' phase comprises a collection of interactive hedonic hotspots, and this hedonic circuitry may be shared by diverse pleasures ranging from sensory food and drug pleasures to human cultural and social pleasures (Berridge and Kringelbach, 2015). Each hedonic hotspot has the special ability, that when neurochemically stimulated, for example by opioids, to amplify 'liking' reactions, thereby making sweetness appear even more enjoyable (Kringelbach and Berridge, 2010). DA stimulations even in hedonic hotspots, by contrast always fail to enhance 'liking' (Smith et al., 2011), indicating the role of DA to be confined to 'wanting'. In support of this theory, studies have shown that 'wanting' and 'liking' could be dissociated in people using alcohol (Hobbs et al., 2005).

Incentive sensitisation is caused in humans has been studies extensively in human. Several studies demonstrated both behavioural and neural sensitisation in humans. For example, the repeated administration of amphetamine in humans has shown to produce persistent behavioural sensitization (e.g. eye-blink responses, vigour and energy ratings), especially at high doses (Boileau et al., 2006; Strakowski and Sax, 1998). Neuroimaging studies have shown that even the oral administration of relatively low doses of amphetamine can produce mesolimbic sensitisation in humans (Boileau et al., 2006; Leyton and Vezina, 2013). Moreover, in addicted individuals mesolimbic brain responses to drug cues, such as viewing photos of drug equipment, are enhanced (Kühn and Gallinat, 2011). Furthermore, repeated use of cocaine and alcohol are 
associated with greater activation to cocaine (Prisciandaro et al., 2014) and alcohol cues (Claus et al., 2011). Hence, there is a need for a more effective neurobiological treatment that would reverse the neuroadaptations underlying sensitized mesolimbic hyper-reactivity to drug cues, without inducing adverse side effects. 


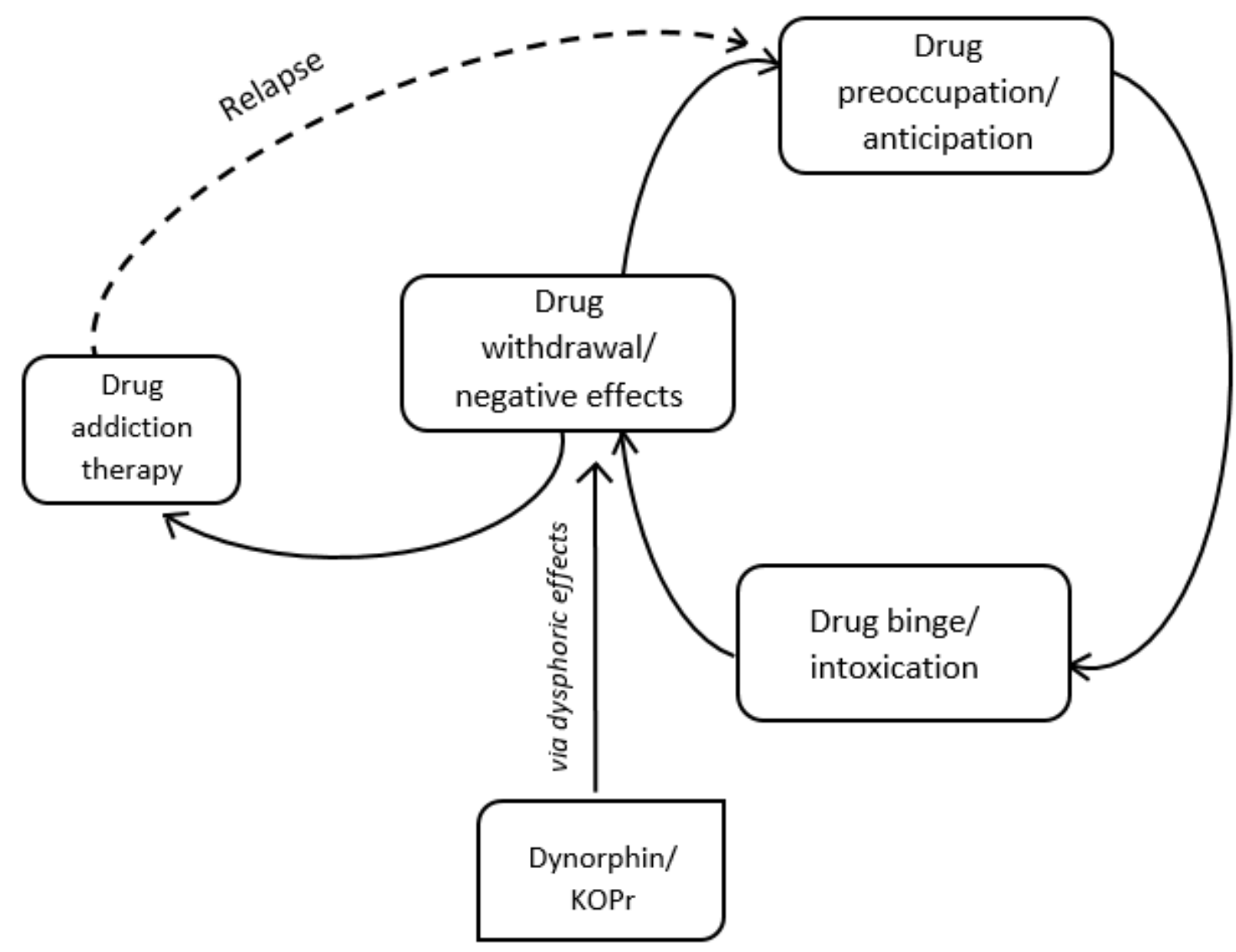

Figure 1.2: An illustration of the three-stage addiction cycle

Preoccupation or 'craving' for the drug promotes drug intoxication, which in turn induces a negative withdrawal state which causes further drug craving to alleviate the negative effects of withdrawal. Addiction therapy attempts to break drug abusers out of the cycle at the withdrawal stage; if unsuccessful, those being treated will often relapse to drug abuse. Drugs taken over time are less rewarding and drugs taken reverse the negative withdrawal effects, which has been attributed to the dynorphin/kappa opioid receptor (KOPr) via dysphoric effects. Figure adapted from Koob and Le Moal $(1997,2001)$ and Shippenberg et al., (2007). 


\subsubsection{Behavioural models of drug addiction}

Due to the complexity of human behaviour in drug addiction, human addiction is difficult to model. However, extensive research in the addiction field has developed animal models with an attempt to mirror this complexity of human behaviour in animals.

\subsubsection{Drug self-administration: Model of drug addiction}

The drug self-administration model is a preclinical model that is widely used to model human addiction in preclinical models. The first use of a drug self-administration procedure was described by Weeks in 1962. In this model, rats were trained to perform an operant task (pressing of the lever) to receive morphine via i.v. infusion into the jugular vein (Weeks, 1962). The drug infusion acts as a positive reinforcer for the required behaviour, eliciting repetition of the tasks (Weeks, 1962). The conditioning of the animals to associate drug infusions with a physical task of a lever press is quickly learned by the animal and, over time, the responses are stably maintained by continual exposure of the rewarding drug to the animal (Weeks, 1962). Drug selfadministration studies have been extensively used and animals have been found to selfadminister many classes of addictive drugs including morphine (Glick et al., 1995; Thompson and Schuster, 1964), heroin (Bonese et al., 1974; Pettit et al., 1984), cocaine (Glick et al., 1995; Pickens and Thompson, 1968), and methamphetamine (Balster and Schuster, 1973; Johanson et al., 1976; Kitamura et al., 2006). In rats, drugs of abuse, such as cocaine, strongly induce self-administration compared to MDMA and nicotine, which are weak reinforcers (Manzardo et al., 2002; Schenk et al., 2007).

Today, self-administration models are considered to be the gold standard for assessing the effects of drugs of abuse preclinically owing to the similarities between human and animal drug use (Carter and Griffiths, 2009; Katz and Higgins, 2003). For instance, in terms of the variables that produce relapse in the reinstatement of drug self-administration model, there are similarities between humans and animals. For example, external stimuli or cues such as equipment, places, visual and auditory characteristics of the environment and the internal stimuli like exposure to drugs, mood states like anxiety or stress reinstate drug seeking behaviour in both animals and humans (Carroll, 1998). A major advantage of animal models used is in the translation of the human condition to the animal models (face validity) and the translation of the neurobiological measures back to the human condition in order to predict vulnerability (predictive validity) 
(Shippenberg and Koob, 2002). For example, naltrexone, an opiate antagonist has been known to block self-administration of alcohol in rhesus monkeys (Altshuler et al., 1980) and male Wistar rats (Stromberg et al., 1998) and this preclinical study eventually led to the use of naltrexone to successfully prevent relapse in detoxified alcoholics (O'Malley et al., 1992). This is the best example of using preclinical models of addiction in the development of medications for the treatment of drug abuse. Thus, self-administration studies have a predictive validity for human drug use, thereby encouraging its use as a tool to study potential pharmacotherapies preclinically (Haney and Spealman, 2008; O'connor et al., 2011).

\subsubsection{Modelling different aspects of addiction and drug-taking behaviours preclinically}

Manipulations of simple drug self-administration models allows evaluation of different aspects of addiction. Inclusion of a chained schedule, where conditioned behaviour is maintained by presenting one or more links in the chain of stimuli that precede delivery of the drug. For instance, animals may be required to complete a physical task for the presentation of a tone, and perform subsequent responses for the tone before resulting in drug delivery (Ferster and Skinner, 1957). Due to the difficulty in maintaining reinforced behaviour by several links, the chained schedule is not commonly utilised. For detailed review of various drug self-administration models see (Panlilio and Goldberg, 2007; Epstein et al., 2006; Dobrin and Roberts, 2012; Schindler et al., 2002) and for review of models of drug craving see (Markou et al., 1993).

A major problem when studying addiction preclinically is relapse to drug use after periods of abstinence (O'Brien, 2005). In drug addicts, drug relapse and craving during abstinence are often triggered by acute re-exposure to the self-administered drug (De Wit, 1996), drug-associated cues and contexts (O'Brien et al., 1992), or certain stressors (Sinha et al., 2011). This clinical scenario has been studied over the last two decades using reinstatement models in laboratory animals. For example, relapse following acute re-exposure to a drug is modelled preclinically by providing a drug prime to rats following drug-extinction and the number of self-administration responses recorded (De Wit and Stewart, 1981). Reinstatement following exposure to cues (tone, light, odour) (Meil and See, 1996), or exposure to stressors (Shaham and Stewart, 1995) can be modelled in similar ways. This phenomenological similarity, as well as the reliability of the 
reinstatement model in detecting reinstatement induced by these stimuli, has led to a dramatic increase in the use of this model in the addiction field (Bossert et al., 2013).

The drug-primed reinstatement model has an extinction and reinstatement phase introduced (De Wit and Stewart, 1981), where, during extinction, access to the drug is removed resulting in a reduction in responses in the absence of the reinforcer. In addition, access to the drug can be paired with cues such as sound, light or odour, which predicts the availability of rewards and instigates the behaviour. Such cues associated with drugs can spur continued drug seeking behaviour and relapse. As the reinstatement model is currently the most commonly used animal model of drug relapse, we investigated the reinstatement of a previously extinguished cocaineseeking behaviour by using an operant self-administration procedure in male Sprague-Dawley rats using established protocols (Ewald et al., 2017; Riley et al., 2014).

The cocaine dose-response model of self-administration is useful to explore the rewarding effects of different doses of cocaine. This is used to evaluate the strength of reinforcement (number of lever presses/infusions) at different drug doses in rodents. The assessment of cocaine reinforcement behaviour generally reveals an inverted U-shaped dose-response curve (Katz et al., 1989; Mello and Negus, 1996). Drugs typically have an optimal rewarding dose with both high and low concentrations being less rewarding than intermediate doses (Hiranita et al., 2009; Mantsch et al., 2007) or it may be that animals response less at higher doses as they would need less amount of the drug to achieve the reward they need. In this study, different cocaine doses were delivered and assessed for the cocaine taking behaviour in rats using a protocol described by Song et al. (2012). 


\subsubsection{Kappa opioid receptor system}

\subsubsection{The endogenous kappa opioid receptor system}

The KOPr is a seven transmembrane GPCR, sensitive to pertussis toxin and coupled to $\mathrm{G}_{\mathrm{i} / \mathrm{o}}$ proteins found throughout the nervous system (Avidor-Reiss et al., 1995). KOPr is encoded by the OPKR1 gene (Butelman et al., 2012) which has been cloned in rat (Li et al., 1993; Meng et al., 1993; Minami et al., 1993), mouse (Yasuda et al., 1993) and human (Mansson et al., 1994; Zhu et al., 1995). More recently, a crystal structure was obtained for human KOPr in complex with the KOPr antagonist JDTic (Wu et al., 2012), and with the KOPr agonist MP1104 (the compound utilised in this study) (Che et al., 2018). The three subtypes of KOPr (KOPr1, KOPr2 and KOPr3) have been identified pharmacologically; however, only one variant has been cloned (Simonin et al., 1995). KOPr is closely related to MOPr and DOPr, with approximately $75 \%$ and $65 \%$ sequence identity in the transmembrane $\alpha$-helices and intracellular loops. However, larger differences are seen in the extracellular loops and $\mathrm{N}$ - and C-termini with only 35-40\% sequence identity (Pogozheva et al., 2005).

KOPr is distributed throughout the human brain and spinal cord, with greater concentrations in the occipital lobe, amygdala, olfactory bulb, NAc, hippocampus, VTA, frontal and temporal lobes, thalamus, cerebral cortex and parietal lobe. It is also found in dorsal root ganglion (DRG) and moderate expression in the lamina II region of dorsal, cervical and lumbar spinal cord regions (Peckys and Landwehrmeyer, 1999; Simonin et al., 1995) (see Figure 1.1 and 1.5 for KOPr expression in reward \& aversion and in pain pathway respectively). KOPr is also found on both pre- and post-synaptic dopaminergic, serotonergic, glutamatergic and $\gamma$-aminobutyric acid (GABAergic) neurons in the brain (Shippenberg et al., 2007; Svingos et al., 2001). However, expression of KOPr in the periphery has not been well characterised. Studies in rats found KOPr in adrenal gland, testis, kidney, ovary, uterus, lung, stomach and both large and small intestines, with no trace of KOPr expression found in liver and low levels in spleen and heart. However, in humans low levels were identified in all the above mentioned tissues, including pancreas, skeletal muscles and thymus (Peng et al., 2012; Sobanski et al., 2014).

The endogenous ligand for KOPr is dynorphin, which is a class of opioid peptide that is a posttranslational product of the PDYN gene (Marinova et al., 2005). Dynorphin is found in the central nervous system (CNS), with high levels in pallidus, substantia nigra (SN), dorsal striatum (dStr), NAc and hypothalamus, whereas moderate levels are found in medulla, pons, hippocampus, periaqueductal grey matter, and amygdala (Gramsch et al., 1982; Watson et al., 1982). The active 
form of dynorphin that activates KOPr is dynorphin $A_{(1-17)}$, and has been shown to have some affinity for MOPr and DOPr (Chavkin et al., 1982). Activation of KOPr is associated with regulation of the reward pathway, anxiogenic and stress-associated behaviour, and anti-nociception (Wang et al., 2010). In addition, KOPr promotes axon myelination (Du et al., 2016; Mei et al., 2016).

\subsubsection{Kappa opioid receptor signalling pathways}

KOPr activation produces different signalling responses depending on the type of ligand administered (agonist or antagonist), length of exposure, the dose administered, and route of administration. A brief summary of the known cellular effects of KOPr activation as well as the commonly activated signalling pathways is shown in Figure 1.3.

When a KOPr agonists bind to the receptor, various downstream signalling pathways are activated. KOPr signalling is regulated via G-proteins, where activation of KOPr ligand leads to

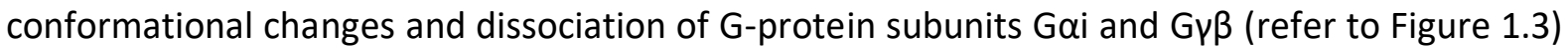
(Bruchas and Chavkin, 2010). Gai protein subunit causes inhibition of cyclic adenosine monophosphate (cAMP) synthesis by adenylyl cyclase (Taussig et al., 1993), whereas G $\gamma \beta$ protein subunit is thought to affect G-protein gated inwardly rectifying potassium channels, causing $\mathrm{K}^{+}$ influx (Grudt and Williams, 1993) and inhibit voltage-gated calcium ion channels, thereby inhibiting $\mathrm{Ca}^{2+}$ influx (Eriksson et al., 1993), the resultant effect causes hyperpolarisation of neuron, thereby decreasing neuronal excitability and inhibiting action-potential transmission (refer to Figure 1.3). The G-protein signalling pathway also leads the phosphorylation of the stress associated c-Jun N-terminal kinase (JNK) (Kam et al., 2004) and to the early phase phosphorylation of extracellular signal-regulated kinase 1 and 2 (ERK1/2), which is dependent on phosphatidylinositol 3-kinase (PI3K), protein kinase $\mathrm{C}(\mathrm{PKC}) \zeta$ and $\mathrm{Ca}^{2+}$ mobilisation (Belcheva et al., 2005). The ERK1/2 has two marked stages known as 'early' and 'late' phase activation. The early phase activation is mediated via $G \gamma \beta$, whereas late phase via $\beta$-arrestin recruitment by KOPr (McLennan et al., 2008). Recruitment of $\beta$-arrestin leads to phosphorylation of p38 mitogenactivated protein kinase (MAPK) and late phase ERK1/2 pathway, which in turn leads to the activation of the transcription factor CAMP response element binding protein (CREB). The actions of p38 MAPK have been attributed strongly in the development of pro-depressive and aversive effects of KOPr agonists (Bruchas et al., 2011; Ehrich et al., 2015). Increased CREB activity ends in 
KOPr mediated pro-depressive effects (Mague et al., 2003) and is an important factor in stressinduced behavioural responses to drugs of abuse (Kreibich and Blendy, 2004). KOPr phosphorylation, and $\beta$-arrestin mediated uncoupling of G-proteins leads to desensitisation of KOPr as G-protein pathway no longer can be activated (Liu-Chen, 2004). Phosphorylation of KOPr leads to internalisation of the receptor by formation of endocytic vesicles, and causing downregulation of the KOPr on the cell surface (Schulz et al., 2002). The internalised receptors are dephosphorylated and are either degraded or recycled back to the plasma membrane (Liu-Chen, 2004). Studies have shown PKC can also lead to the phosphorylation of KOPr both as agonist dependent and-independent manner (Chiu et al., 2017). More recently, it has been shown that $\mathrm{KOPr}$ agonist tolerance and internalisation is contributed by G-protein receptor kinase (GRK) mediated phosphorylation (Chiu et al., 2017; McLaughlin et al., 2004). The downstream functions of $\beta$-arrestin recruitment extensively obstruct the therapeutic application of KOPr agonists, both as analgesic agents and potential anti-addiction therapies (Bruchas and Chavkin, 2010). The idea that an agonist could be designed to activate the G-protein pathway preferentially without activating the $\beta$-arrestin-dependent pathway is termed as functional selectivity or biased agonism (discussed in detail in section 1.2.6.3). Although the idea of biased agonism is gaining a lot of attention (Rankovic et al., 2016; Spetea et al., 2017; Viscusi et al., 2016; White et al., 2015), there still remains gaps to fully understand this concept due to the complexity in downstream signalling pathways. For example, a derivative of Sal A , RB-64 $(3 \mathrm{mg} / \mathrm{kg}$ ) delivered via subcutaneous (s.c.) injection produced potent analgesic effects in mice, measured using the hotplate assay (White et al., 2015). The sedative effects that RB-64 had in tests of motor coordination (measured using rotatrod performance test) were mediated via $\beta$-arrestin. However, the aversive effects (evaluated in conditioned place aversion (CPA) tests) were present in both wild-type and $\beta$ arrestin knock out $(\mathrm{KO})$ mice indicating that the aversion is partly regulated through G-protein activation (White et al., 2015). 
G-protein signalling pathway

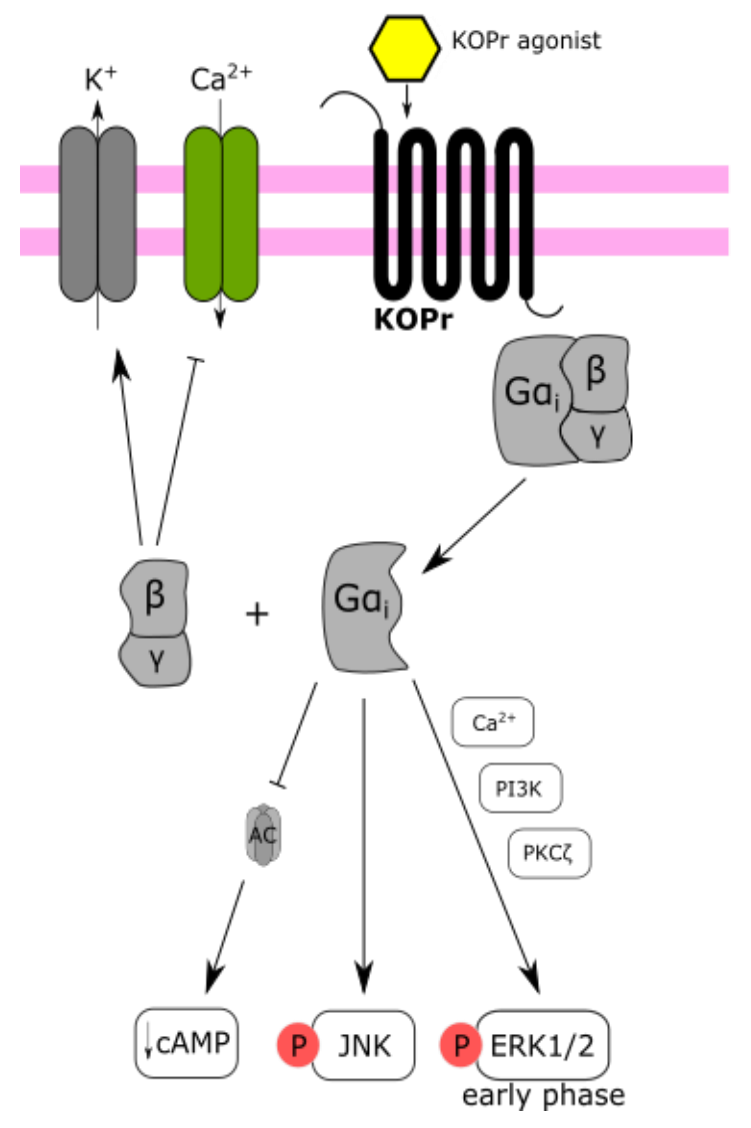

Anti-addiction effects

Analgesic effects $\beta$-arrestin signalling pathway

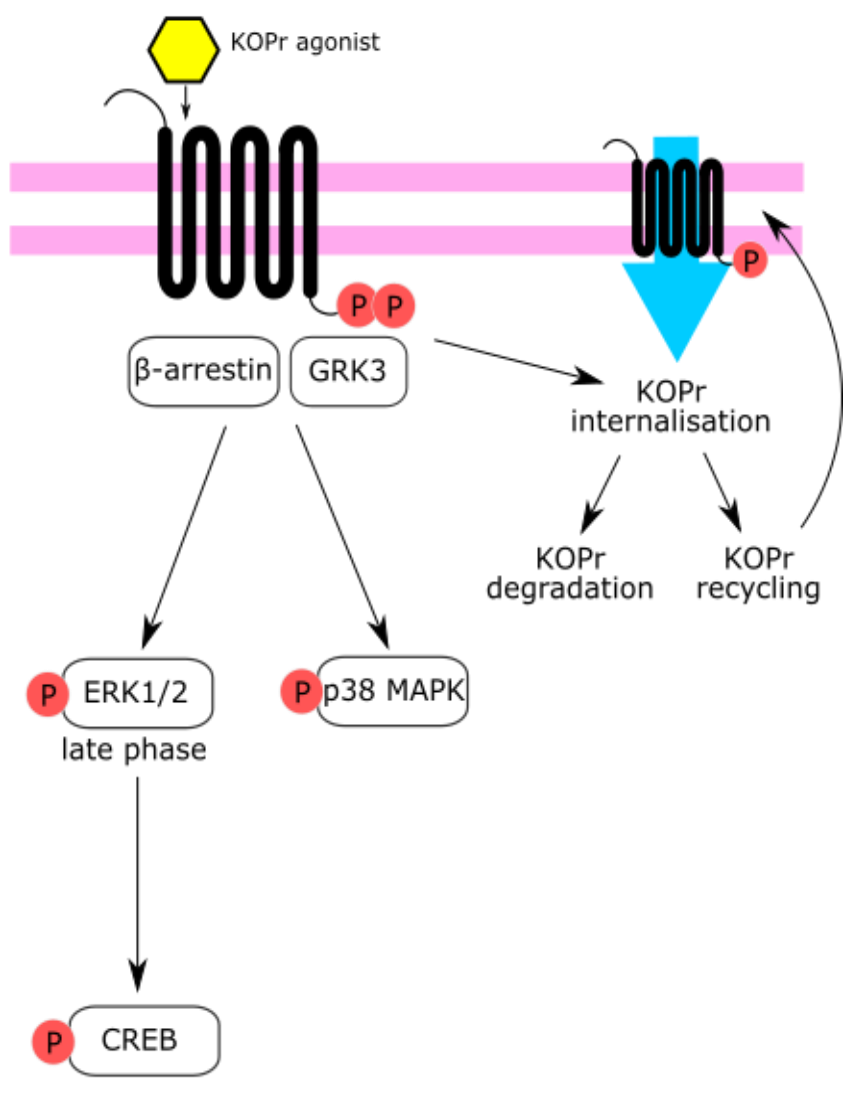

Aversive effects

Depressive effects

\section{Figure 1.3: Kappa opioid receptor mediated signalling pathways}

KOPr agonist binding results in the activation of G-protein and $\beta$-arrestin signalling pathways. Activation of G-protein signalling pathway results in the dissociation of the G-protein complex from the receptor. The dissociated the $\mathrm{G} \alpha_{i}$ subunit inhibits adenylyl cyclase (AC) activity leading to reduction in cAMP levels within the cytoplasm. The $G \alpha_{i}$ subunit phosphorylates (red circle) second messengers such as ERK1/2 and JNK. The $\beta \gamma$ subunits activate the G-protein-gated inwardly rectifying potassium $\left(\mathrm{K}^{+}\right)$and inhibits the voltage gated calcium ion channel $\left(\mathrm{Ca}^{2+}\right)$, resulting in hyperpolarisation of the neuron. The $\beta$-arrestin-dependent pathway includes the phosphorylation of the intracellular domain of the KOPr by GRK3 and $\beta$-arrestin recruitment to 
the receptor, clathrin-mediated receptor internalisation within endosomes, followed by either degradation via lysosomes or recycling back to the plasma membrane. This signalling pathway leads to a late-phase phosphorylation of ERK1/2, p38 MAPK and CREB, resulting in the aversive and depressive effects. Figure adapted from Bruchas and Chavkin (2010) and Chavkin (2011). Arrows refer to activation.

CAMP, cyclic adenosine monophosphate; AC, adenylyl cyclase ; ERK1/2, extracellular signal-

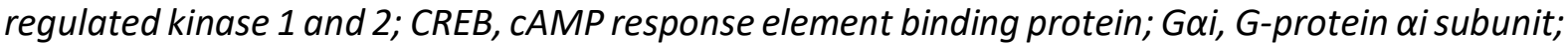

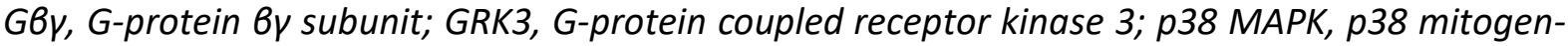
activated protein kinase; PI3K, phosphoinositide 3-kinase; $P K C \zeta$, protein kinase C $\zeta$. JNK, c-Jun Nterminal kinase. 


\subsubsection{Functional selectivity at the kappa opioid receptor}

While many strategies have been explored to mitigate the side effects of KOPr agonists, the most popular recent concept includes the development of G-protein biased agonists (Kenakin, 2007; Kenakin and Christopoulos, 2013; Urban et al., 2007), due to the fact that $\beta$ arrestin-mediated signalling is largely responsible for the negative side effects exerted by KOPr agonists (Bruchas and Chavkin, 2010; Bruchas and Roth, 2016; Chavkin et al., 2014).

Agonist designed to preferentially activate G-protein signalling without the recruitment of $\beta$ arrestin signalling is termed as G-protein biased. It has been contemplated that the beneficial therapeutic effects associated with KOPr activation such as anti-nociception and anti-addiction effects are the result of G-protein mediated pathway, whereas the side effects of KOPr agonists are due to $\beta$-arrestin pathway (Bruchas and Chavkin, 2010; Kivell et al., 2014b). The adverse effects have been associated with late phase ERK1/2 phase, which is connected to $\beta$-arrestin recruitment, which in turn leads to downstream responses like activation of p38 MAPK and CREB (see Figure 1.3), both of which are associated in dysphoria and depression when KOPr is activated (Carr and Mague, 2008; Pliakas et al., 2001). The phosphorylation of ERK1/2 and p38 MAPK can be assessed using specific antibodies recognising the phosphorylated form of ERK and western blotting techniques. One such assessment in Kivell laboratory revealed that the KOPr agonist 16bromosalvinorin A (16-Bromo Sal A), an analogue of Sal A showed no activation of ERK1/2 or p38 MAPK in rat dStr and pFC, but in the NAc region significant phosphorylation of p38 MAPK alone was noticed (Ewald, 2016). In support of this, 16-Bromo Sal A (1 mg/kg, i.p.) when screened for side effect profile, it did not produce sedation (Riley et al., 2014), anxiety (Ewald, 2016) or depression (Ewald, 2016) in respective spontaneous locomotor tests, elevated plus maze (EPM) or light-dark test, and forced swim test (FST). This indicates that 16-Bromo Sal A may be acting preferentially through the G-protein biased pathways. However, 16-Bomo Sal A was found to be aversive in CPA tests, indicating that the resultant effect could be due to p38 MAPK activation, as aversive effects have been shown to be regulated via p38 MAP kinase signalling pathways (Bruchas et al., 2007; Ehrich et al., 2015; Zan et al., 2016). A few biased agonists have been developed (Spetea et al., 2017; Viscusi et al., 2016; White et al., 2015).

The application of the theoretical idea of biased agonism, however, is complex. The complicated understanding of signalling pathways and the technical differences in measuring and calculating bias have slowed progress in identifying potentially biased therapeutics (Michel and Charlton, 
2018). Crowley et al. (2016) showed that bias can be calculated using potency and efficacy that results from both $\mathrm{G}$-protein and $\beta$-arrestin assays using the following formula:

$\log ($ bias factor $)=\log \left(\frac{E_{\max (\text { test })} \times E C_{50(\text { control })}}{E C_{50(\text { test })} \times E_{\max (\text { control })}}\right)_{\beta \text {-arrestin }}-\log \left(\frac{E_{\text {max }(\text { test })} \times E C_{50(\text { control })}}{E C_{50(\text { test })} \times E_{\max (\text { control })}}\right)_{G-\text { protein }}$

Control compound is a balanced agonist with same potency in both assays (example: U50,488 (Schattauer et al., 2017); Sal A (White et al., 2015); dynorphin $A_{(1-17)}$ (DiMattio et al., 2015) have been previously set as control compounds). A bias factor of 1 is a balanced agonist, less than 1 is G-protein biased and more than 1 is $\beta$-arrestin biased. It is noteworthy that the bias factor in different species varies and this further complicates the bias calculations. Studies found U50,488 was biased towards $\beta$-arrestin in mouse KOPr but unbiased at the human KOPr. The clinically available KOPr agonist, nalfurafine was found to be more G-protein biased at human KOPr but to a less extent at the rat KOPr (Schattauer et al., 2017). Hence, there is a need to study various cell signalling pathways to better understand the biased agonism concept. 


\subsection{The link between pain and addiction}

There exists a connection between chronic pain and addiction, as untreated pain can result in drug seeking and other behaviours that lead to addiction (Cheatle et al., 2014). Considering the current impact of the opioid crisis globally, there is a need for developing non-addictive opioid analgesics. Hence, this thesis also looked in the anti-pain properties of our novel mixed opioid agonist in animals. As mixed opioid agonists have been suggested to have safer therapeutic profile than selective MOPr, KOPr or DOPr agonists (Anand et al., 2016; Majumdar and Devi, 2018), we hypothesise that our novel mixed opioid agonist will be useful in both, pain and addiction.

\subsubsection{Prevalence and socioeconomic cost}

Chronic pain is a growing public health concern and has been linked to reduced quality of life (Gureje et al., 1998; Smith et al., 2001). Chronic pain is connected to numerous physical and health conditions with high risk for increased rates of anxiety and depression (Dahlhamer et al., 2018) and mortality (Torrance et al., 2010). In addition, chronic pain is also associated with dependence on opioids by its sufferers (Simon, 2012).

The increase in number of chronic pain sufferers has become a worldwide concern, contributing to high health care costs and lost productivity. In New Zealand, it is been reported that one in six adult New Zealanders suffer from chronic pain (Dominick et al., 2011). A recent survey by the New Zealand Ministry of Health in the year 2015/16 found that 21\% of New Zealand adults suffer from chronic pain lasting longer than 6 months (Cox, 2016). In addition, in countries like Australia, the USA and the United Kingdom, the prevalence of chronic pain was found to be $19 \%$ (Henderson et al., 2013), 11-40\% (Kuehn, 2018) and 35-51\% (Fayaz et al., 2016) respectively. Chronic pain was also reported to occur in 19\% (Breivik et al., 2006) and 11\% (Ng et al., 2002) of adult European and Asian population respectively. Moreover, in USA alone, chronic pain contributes to an estimated $\$ 560$ billion annually with costs associated in medical expenses and lost of productivity (Kuehn, 2018). Hence there is a need to address this globally rising problem. 


\subsubsection{Physiology of pain}

Pain is described by the International Association for the Study of Pain as "an unpleasant sensory and emotional experience associated with actual or potential damage" (IASP Task Force on Taxonomy, 1994). The physiology of pain is the transmission of painful stimuli modulated by a complex network of sensory and descending inhibitory neurons (Bourne et al., 2014; Marchand, 2008) (see Figure 1.4). The physiologic component of pain, called nociception, includes modulation, transduction and transmission of signals produced in response to a noxious stimulus (Lamont et al., 2000). Nociceptors are defined as 'a peripherally-localized receptor sensitive and responding to noxious stimuli' (Millan, 1999). They recognise the noxious stimuli in the skin including viscera, joints and muscles (Chouchkov, 2012). The primary afferent neuron transmits the pain signal generated by nociceptor activation. There are two classes of nerve fibres that transmit pain signal by nociceptors, they are the myelinated $A \delta$ fibres, which conducts impulses quickly $(6-25 \mathrm{~m} / \mathrm{s})$ due to thinly myelinated axons, and mediates the first phase of pain which is a sharp pain (Julius and Basbaum, 2001). Whereas unmyelinated C fibres, conduct impulses at a comparatively slower rate, (approx. $1 \mathrm{~m} / \mathrm{s}$ ) (Julius and Basbaum, 2001). A $\delta$ fibres are associated with mechanical and thermal nociceptors, and $C$ fibres are associated with inflammation or damaged tissue (Kidd and Urban, 2001). 


\section{(A)}

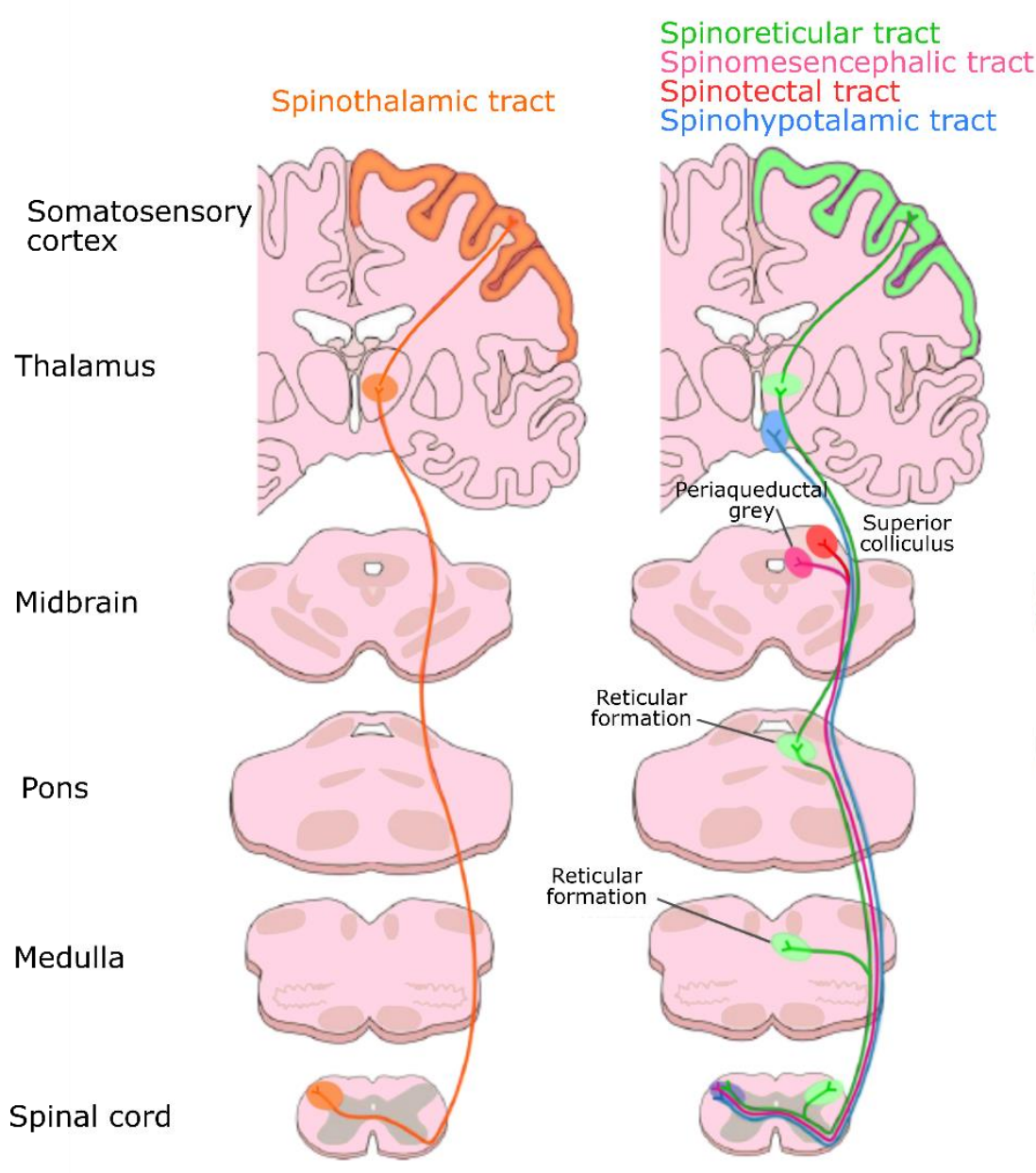

(C)

Descending pathway

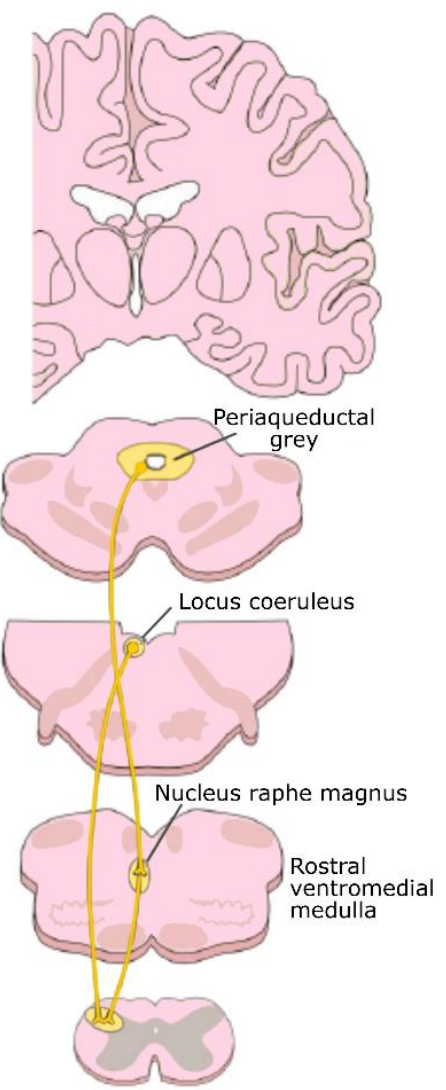

\section{Figure 1.4: Ascending and descending pain pathways}

(A) The spinothalamic tract is a major pathway for the transmission of pain. It relays information from the dorsal horn of the spinal cord to the thalamus and the somatosensory cortex. (B) The other ascending pathways involved in pain processing are the spinoreticular tract, the spinomesencephalic tract, the spinotectal tract and the spinohypothalamic tract. (C) The descending pathway assists in the modulation of pain beginning in the periaqueductal grey in the midbrain and extends to the nucleus raphe magnus in the rostral ventromedial medulla and finally to the dorsal horn of the spinal cord. Figure adapted from Kandel et al. (2000). 


\subsubsection{Classification of pain}

Pain may be classified as nociceptive, inflammatory and neuropathic pain based on the underlying mechanisms. A complete overview of pain is beyond the scope of this thesis, for a complete overview of pain see (Twycross, 1997; Woolf, 2004; Woolf et al., 1998; Woolf and Costigan, 1999) (reviewed in Woolf (2010); Kuner (2010); Baron et al. (2010) and Ji et al. (2014)). Here I discuss both nociceptive and neuropathic pain, which were evaluated in this thesis using the warm-water tail withdrawal and chemotherapy-induced neuropathic pain (CINP) preclinical models respectively.

\subsubsection{Nociceptive pain}

Nociceptive pain is caused by injury and plays a protective role (Milligan and Watkins, 2009). An example of nociceptive pain is a cut or burn to the skin (Woolf, 2010). A common model to access nociceptive pain is the tail withdrawal assay, which measures the spinal withdrawal reflex in response to painful stimuli, such as heat commonly applied as hot water or an infrared beam (Dykstra and Woods, 1986). The increased latency to withdraw the tail from a thermal stimulus indicates an anti-nociceptive effect of an administered drug. This model involves reflexes modulated by the brain stem and spinal cord (Roberts and Rees, 1986), and does not involve supra-spinal cortical brain pathways (Irwin et al., 1951).

To measure the supra-spinal reflex, the hotplate test, another thermal nociceptive pain model is used (Casey et al., 1996). The typical hotplate test model involves measuring the response time for the animal to lick or withdraw paw after placing the animal in a heat-conducting surface (Saadé and Jabbur, 1985).

\subsubsection{Neuropathic pain}

Neuropathic pain is characterised by continuous spontaneous pain and is typically described as burning, aching or shooting in nature (Selph et al., 2011). This pain results from malfunctioning of the nervous system, and serve no protective function. The classical examples of such pain include persistent pain following spinal cord injuries, chemotherapy treatment, and in multiple sclerosis (Kuner, 2010). Neuropathic pain can occur following nerve damage in both the peripheral nervous system (PNS) or CNS. Common animal model used to model this pain include ligation of the sciatic nerve (Bennett and Xie, 1988). Neuropathic pain can also be induced by chemotherapy agents 
and CINP is a common side effect in chemotherapy-treated cancer patients. Chemotherapeutic agents such as platinum derivatives (cisplatin, oxaliplatin), vinca alkaloids (vincristine) and taxanes (paclitaxel) have all been shown to induce neuropathic pain (Jaggi et al., 2011) and animal models of CINP have been developed using these chemotherapy agents and the resulting hypersensitivity to mechanical and cold stimuli is measured to evaluate CINP in animals (Dougherty et al., 2004).

In this study, CINP was induced in male mice using paclitaxel, a taxane based chemotherapy drug used to treat breast, lung and ovarian cancers (Kudlowitz and Muggia, 2013). Cells treated with paclitaxel arrest at metaphase, ceasing progression into anaphase, leading to inhibition of mitosis and finally causing apoptotic cellular death (Weaver, 2014). The specific mechanism of action of paclitaxel-induced neuropathic pain is not completely defined, with various underlying mechanisms published. The pathogenesis of paclitaxel-induced neuropathic pain involves peripheral nerve demyelination and axonal damage, causing distal sensory axons to degenerate in the PNS, and sensitisation of nociceptive afferents, leading to neuropathic pain symptoms (Dougherty et al., 2004). Neurotoxic substance like paclitaxel causes neuropathy of DRG due to the lack of efficient neurovascular blood-brain barrier in DRG (Cavaletti et al., 2000), thus allowing easy penetration. Further validation of the mechanism of action of paclitaxel is required.

There are no effective treatments to treat chronic neuropathic pain (Hershman et al., 2014). CINP is chronic in nature and may persists for months following cessation of chemotherapy treatment, and causes a negative impact on patients (Mols et al., 2014). Hence, there is need for developing effective therapeutics for treating cancer pain.

\subsubsection{Kappa opioid receptor agonists for pain treatment}

Spurred by the need for an efficacious non-addictive analgesic, new research has provided insight for the development of effective KOPr and DOPr agonists to overcome these obstacles (Vanderah, 2010). KOPr agonists are a promising alternative for developing non-addictive analgesics. Traditional KOPr agonists such as U50,488 and U69,593, have been studied extensively for antinociceptive effects and have shown to attenuate nociceptive processes (Chang et al., 1989; Randic et al., 1995). U50,488 showed significant anti-nociceptive effects in thermal, mechanical and visceral models in mice and rats (Vonvoigtlander et al., 1983). Vonvoigtlander et al. (1983) studied the anti-nociceptive effects of U50,488 compared to morphine analgesia in mice and rats in various thermal, pressure and irritant anti-nociceptive assays. In rats, U50,488 showed anti- 
nociceptive effects in warm plate, hot plate and air writhing assay, however this study showed morphine to be more potent compared to U50,488 (Vonvoigtlander et al., 1983). The same study also found similar effects in mice, with U50,488 and morphine showing anti-nociceptive effects in hydrochloric acid writhing test, tail pinch and the tail withdrawal model, however morphine had potent anti-nociceptive properties compared to U50,488 (Vonvoigtlander et al., 1983). U69,593 (i.c.v.) administration has also shown anti-nociceptive effects in the tail withdrawal $\left(55^{\circ} \mathrm{C}\right)$ test in mice which was nor-BNI reversible, indicating the anti-nociceptitve effects were mediated by KOPr (Horan et al., 1991).

Sal A and structural analogues, activating the KOPr have shown anti-nociceptive effects in many pain assays, however, the short duration of action (Johnson et al., 2011) significantly hampers their clinical development. Thus, effective anti-nociceptive agents with a longer duration are highly sought after. This thesis explores the analgesic potential of a longer acting mixed opioid compound, MP1104 to determine whether these properties are more effective in assays of both nociceptive pain and CINP with fewer side effects. 

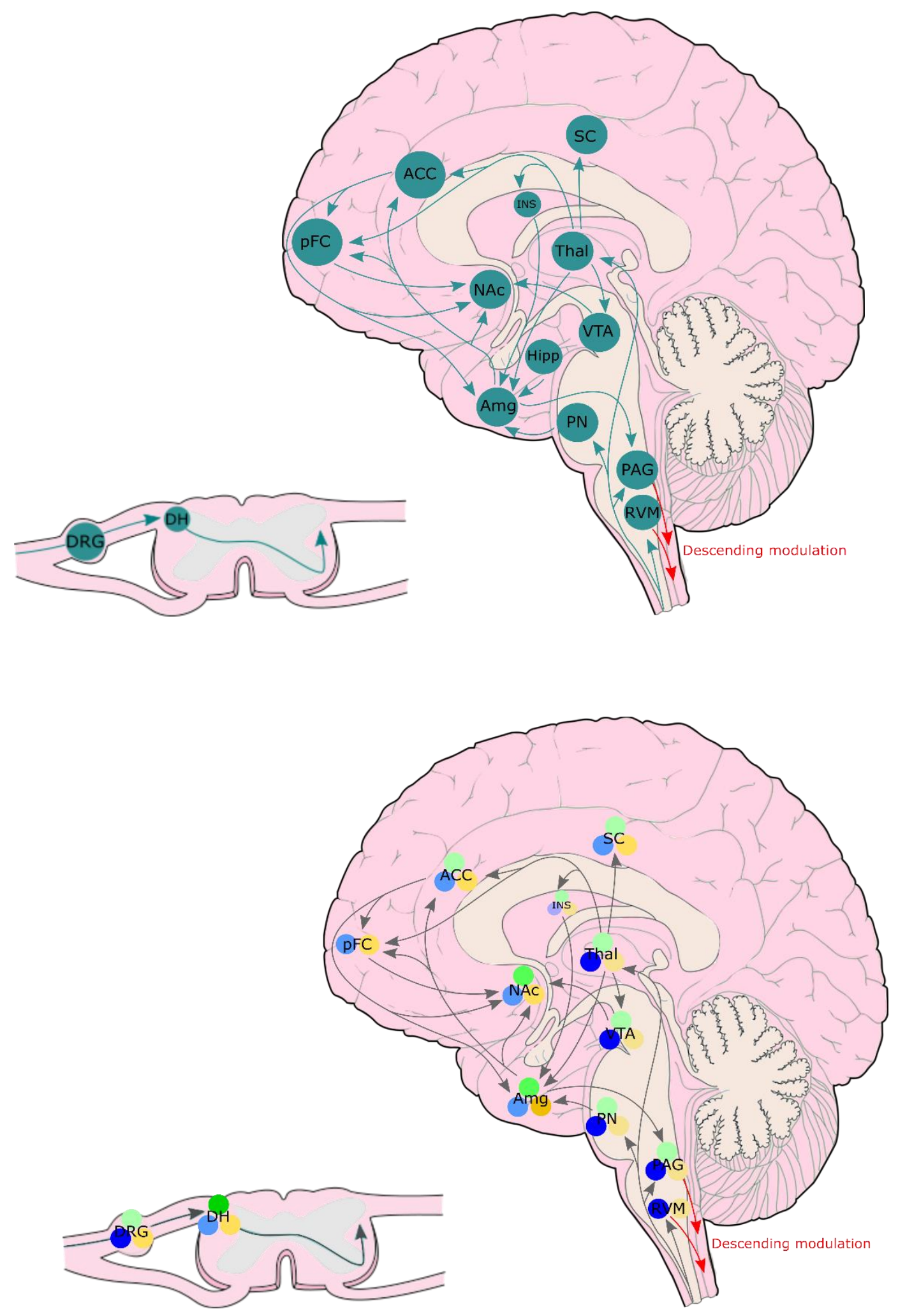

Figure 1.5: Regions of brain and spinal cord involved in pain processing compared to kappa, mu and delta opioid receptor expression

A large interconnected neural network of the brain circuits involved in pain processing and perception ascending from spinal cord to the supraspinal brain regions. Kappa opioid receptor 
(KOPr) (green circle), mu opioid receptor (MOPr) (blue circle) and delta opioid receptor (DOPr) (yellow circle) expression in relevant areas of pain are shown (darker colour indicates greater expression of KOPr, MOPr or DOPr). Figure adapted from Corder et al. (2018).

$A C C$, anterior cingulate cortex; Amy, amygdala; $D H$, dorsal horn of the spinal cord; DRG, dorsal root ganglion; Hipp, hippocampus; INS, insula; NAc, nucleus accumbens; PAG, periaqueductal grey; pFC, prefrontal cortex; PN, parabrachial nucleus; RVM, rostral ventromedial medulla; SC, somatosensory cortex; Thal, thalamus; VTA, ventral tegmental area. 


\subsection{Side effects of traditional kappa opioid receptor agonists}

Previously, studies have reported that selective KOPr agonists are involved in stress, fear, anxiety, and depressive-like behaviours (Bruchas et al., 2010; Gillett et al., 2013; Knoll and Carlezon, 2010). KOPr agonists have been shown to cause sedation, aversion, anxiety and depression in animals (Mello and Negus, 2000; Carlezon et al., 2006; Valdez and Harshberger, 2012; Sufka et al., 2014) (discussed in detail below; also see Table 4.1). These side effects limits their clinical use.

\subsubsection{Sedation}

Many studies have evaluated the sedative effects of a variety of KOPr agonists preclinically. In rats U50,488 (2.5-40 mg/kg, s.c) showed presence of sedative effects in rotarod test (Gallantine and Meert, 2008; Wang et al., 2009), whereas Sal A had no effect (0.125-2 mg/kg, i.p.) in locomotor activity test (Carlezon et al., 2006; Morani et al., 2009). In mice U50,488 (1, $10 \mathrm{mg} / \mathrm{kg}$, i.p.) (Paris et al., 2011) produced sedative effects in locomotor activity tests, also both U69,593 $(1 \mathrm{mg} / \mathrm{kg}$, i.p.) and Sal A (0.5 - $2 \mathrm{mg} / \mathrm{kg}$, i.p) (Fantegrossi et al., 2005) showed sedation in inverted screen performance test which is used to assess motor effects; however Sal A at lower doses $(0.001-1$ $\mu \mathrm{g} / \mathrm{kg}$, s.c.) showed no effect in the same assay (Braida et al., 2009). In rhesus monkeys U50,488 (0.1 mg/kg/h/i.v.), U69,593 (0.01, $0.056 \mathrm{mg} / \mathrm{kg} /$ i.v.) and Sal A (0.032 - $0.1 \mathrm{mg} / \mathrm{kg} /$ i.v.) showed significant sedative effects (Butelman and Kreek, 2001; Butelman et al., 2009; Negus et al., 1997). Often these effects were seen at doses that were required to exert desirable effects, such as U50, 488 which produced sedative effects (2.5-40 mg/kg, s.c) (Gallantine and Meert, 2008), at doses that were shown to reduce cocaine-primed reinstatement of drug seeking in rats ( $30 \mathrm{mg} / \mathrm{kg}$, i.p.) (see Table 4.1).

\subsubsection{Aversion}

Aversion is one of the side effects mediated via KOPr activation (Suzuki et al., 1992b). CPA is common model used to assess these properties preclinically (Bruchas and Chavkin, 2010; Bruchas et al., 2007; Chefer et al., 2013; McLaughlin et al., 2006; Tejeda et al., 2013; Wee and Koob, 2010; White et al., 2015). The conditioning phase promotes the animal to establish an association between the drug effects and the environmental conditions that the animal is placed into, such as the smell of the chamber, colour and texture of flooring. If an animal finds the drug effects to be pleasant then it spends more time in that chamber, however, if it dislikes the drug effects then 
it spends less time in that chamber. The aversive effects are measured based on the decreased amount of time spent by the animal in the drug-paired chamber. Previously U50,488 $(1 \mathrm{mg} / \mathrm{kg}$, s.c.) (Göktalay et al., 2006) and U69,593 (0.32 mg/kg, s.c) (Tejeda et al., 2013) and Sal A (0.3 -1 $\mathrm{mg} / \mathrm{kg}$, i.p.) (Sufka et al., 2014) have been shown to produce aversive effects in rats and in mice (Chefer et al., 2013; Skoubis et al., 2001; Zhang et al., 2005) (see Table 4.1).

It is well established that agonists acting at the DOPr produce positive reinforcement, whereas KOPr agonists show aversion (Le Merrer et al., 2009). Hence, one way of overcoming the aversivelike adverse effects observed in selective KOPr agonists is the addition of DOPr activity and thereby counteracting the effects. MP1104 is one such compound with mixed KOPr and DOPr agonist actions.

\subsubsection{Anxiety}

KOPr activation has been shown to induce stress and anxiety-like effects in mice (Vunck et al., 2011) and rats (Harshberger, 2010). Clinically, it has been reported that a KOPr agonist, Mr2033 intensified anxiety in male healthy volunteers which was assessed by a psychopathological evaluation scale (Pfeiffer et al., 1986). Also, preclinically KOPr agonists (Braida et al., 2009; Gillett et al., 2013) have been shown to cause anxiogenic effects whereas KOPr antagonists (Carr and Lucki, 2010; Knoll et al., 2007) produced anxiolytic effects, indicating the role of KOPr in modulating anxiety.

Previously, studies have reported that selective DOPr agonists have both anxiolytic and antidepressant effects (Saitoh et al., 2004; Vergura et al., 2008). However, KOPr's are involved in stress, fear, anxiety, and depressive-like behaviours (Bruchas et al., 2010; Knoll and Carlezon Jr, 2010).

A commonly used preclinical model to assess anxiety in animals is the EPM. In this model an animal's exploratory behaviour and its natural tendency to avoid open or elevated spaces are examined (File et al., 2004). Previously anxiety-like behaviour was assessed for KOPr agonists like U50, 488 (5, $10 \mathrm{mg} / \mathrm{kg}$, i.p.) and Sal A (0.3, $1 \mathrm{mg} / \mathrm{kg}$, i.p.) in rats (Ewald et al., 2017; Gillett et al., 2013; Valdez and Harshberger, 2012) and mice (Bruchas et al., 2009; Smith et al., 2012) using this model (see Table 4.1). Also, Sal A produced anxiogenic effects in the light and dark test (Ewald, 2017), another preclinical model used to assess anxiety-like side effects. In this study, our aim was 
to measure the anxiety-like effects of MP1104 in EPM in order to assess whether these adverse effects are attenuated by MP1104 while maintaining potentially therapeutic anti-cocaine effects.

\subsubsection{Depression}

Dynorphin mediated aversion, stress and depressive effects result from KOPr activation (Chartoff et al., 2012; Shirayama et al., 2004). The anti- or pro-depressive effects of KPOr agonists can be quantified using preclinical behavioural tests. FST is a widely used in vivo model to measure these effects in a laboratory setting. The animals' natural instinct is to escape from the environment its put in, however the animal learns that escape is not possible (Castagné et al., 2010). Rats treated with U69,593 (0.3-10 mg/kg, i.p.) have shown immobile behaviour, indicating a depressive-like state (Mague et al., 2003). Similarly, U50,488 has shown to cause depressive-like effects in mice (Zhang et al., 2015). Sal A showed increased depressive-like effects at higher doses (0.25 - 2 $\mathrm{mg} / \mathrm{kg}$, i.p.) (Morani et al., 2009) but at lower doses (10 - $1000 \mu \mathrm{g} / \mathrm{kg}$, s.c.) (Braida et al., 2009) the effects were reduced in animals in the FST assay used. Moreover in tail suspension and chronic mild tests (preclinical assays to measure depressive-like side effects), Sal A showed reduced prodepressive like effects in mice and rats (Braida et al., 2009; Harden et al., 2012). However, in intracranial self-stimulation assay in rats, Sal A showed mixed results with no effect at lower doses (0.25 mg/kg, i.p.) (Ebner et al., 2010), increased and decreased effects at a dose range of 1-4 $\mathrm{mg} / \mathrm{kg}$, i.p. (Carlezon et al., 2006; Potter et al., 2011). In the same assay both U50,488 and U69,593 showed pro-depressive effects in rats and mice (DiNieri et al., 2009; Bonano et al., 2014) (see Table 4.1). In contrast, KOPr antagonists like JDTic, 5'-guanidinonaltrindole (GNTI), nor-BNI, and 5 '-acetamidinoethylnaltrindole showed decreased immobility as observed in FST rats (Beardsley et al., 2005; Carr et al., 2010). Taken together these studies indicate the function of KOPr in mediating depression. In order to obtain a clinically beneficial therapeutic devoid of depressive effects, MP1104 was assessed for pro-depressive effects.

One example of a KOPr agonist that has been clinically developed is Nalfurafine (also called TRK820 and the trade-name Remitch). Its ability to modulate itch at doses that do not have other side effects (Nakao et al., 2016; Ueno et al., 2013) has led to the clinical availability of the drug to treat haemodialysis-induced pruritus (pruritus or itch in patients with chronic kidney disease undergoing haemodialysis) (Kumagai et al., 2010). This highlights the proof of concept of developing such a drug that at a dose that produces the therapeutic effect is devoid of any side effects. Thus, the therapeutic effects and side effects can occur at different doses. One of the aim 
of this study is to determine whether this is the case for MP1104. Hence, at the same doses that would show therapeutic effect would also be evaluated for side effects like sedation, aversion, anxiety and depression. 


\subsection{Mixed opioid receptor agonists}

Opioids are a group of analgesic agents commonly used in clinical practice (Pathan and Williams, 2012). The opioid receptor class comprises of three classical opioid receptors MOPr, KOPr, DOPr and other less known nociception receptor (NOPr) (Feng et al., 2012). The opioid receptors are complex in their functions. Most clinically relevant opioid analgesics bind to MOPr in the CNS and the PNS in an agonist manner to elicit analgesia (Vallejo et al., 2011), whereas KOPr agonists have been shown to play a critical role in regulating the reward system (Di Chiara and Imperato, 1988b). Studies reported that acute administration of KOPr agonists show anti-addictive potential (see Table 1.1) and have anti-nociceptive (Vonvoigtlander et al., 1983), anti-inflammatory (BileviciuteLjungar et al., 2005; Binder et al., 2001), and antipruritic effects (Akiyama et al., 2015; Kumagai et al., 2010). DOPr agonists, on the other hand, were found to have analgesic (Brainin-Mattos et al., 2006; Codd et al., 2009; Gavériaux-Ruff et al., 2008; Jones et al., 2009) and anti-depressant and anxiolytic effects (Perrine et al., 2006; Saitoh et al., 2004; Vergura et al., 2008) (see section 1.5.1 for details on DOPr agonists).

Although the opioid receptor agonists have been shown to have promising therapeutic properties, adverse effects associated with specific receptor activation limits effective treatment. For example, MOPr agonists are known to produce adverse effects such as respiratory depression (Dahan et al., 2001), tolerance (Chu et al., 2006), dependence and addiction (Compton and Volkow, 2006), and KOPr agonists cause dysphoria, sedation and nausea in humans and animals (Land et al., 2008; Mello and Negus, 2000; Pfeiffer et al., 1986), whereas DOPr agonists are found to produce seizures (Bilsky et al., 1995; Comer et al., 1993; Jutkiewicz et al., 2006; Lutz and Kieffer, 2013a). An approach to overcome the undesirable effects of MOPr, KOPr, or DOPr agonism is by targeting multiple opioid receptors simultaneously. Studies have shown that mixed opioid receptor agonists and/or antagonists may be a viable strategy to generate a more desirable drug profile (Anand and Montgomery, 2018; Balboni et al., 2002; Majumdar and Devi, 2018; Váradi et al., 2016). It has been well documented that MOPr and DOPr agonists are rewarding (Shippenberg et al., 2008), whereas, agonists with an affinity at KOPr are aversive (Castro and Berridge, 2014; Suzuki and Misawa, 1997). Hence, it can be suggested that a KOPr ligand in combination with MOPr or DOPr ligand may be an ideal candidate to possess anti-addiction properties, whereby the rewarding properties of either MOPr or DOPr can be balanced by the aversive like effects produced by KOPr agonists. Moreover, although MOPr agonists are excellent in treating severe 
pain compared to KOPr and DOPr opioid agonists, life-threatening side effects caused by MOPr are a major clinical concern.

\subsubsection{Delta opioid receptor agonists}

Another candidate for the development of anti-pain and anti-addiction medications has resulted in the evaluation of DOPr agonists. DOPr agonists have been shown to be effective against persistent and neuropathic pain in mouse, rat and monkey models (Gavériaux-Ruff and Kieffer, 2011; Kabli and Cahill, 2007). DOPr agonists weakly modulate acute nociception compared to MOPr agonists. However, genetic approaches have supported the importance of DOPr in chronic pain, such as neuropathic and inflammatory pain (Gavériaux-Ruff et al., 2008; Nadal et al., 2006). Chronic pain induced by inflammatory or neuropathic pain was reported to increase the analgesic efficacy of DOPr agonists (Kabli and Cahill, 2007; Pradhan et al., 2013), suggesting chronic pain up-regulates DOPr. Factors associated with this pathological condition such as bradykinin and arachidonic acid (Patwardhan et al., 2005) may prime DOPrs and increase receptor function (Rowan et al., 2009). Hence, DOPr agonists have been suggested to be a promising alternative to MOPr analgesics in the treatment of chronic pain (Cahill et al., 2007; Zhang et al., 2006). The association between DOPr system and depression have been evaluated preclinically. Filliol et al. (2000) were the first to demonstrate that deletion of DOPr produces pro-depressive like behaviours in mice. Following this study, several DOPr agonists have been evaluated for antidepression like effects in animal models of depression and have shown to significantly attenuate depressive-like effects in rodents (Broom et al., 2002; Jutkiewicz, 2006; Saitoh et al., 2004; Tejedor-Real et al., 1998; Torregrossa et al., 2006). Thus, clinical trials have been initiated to assess the effectiveness of DOPr agonists, ADL5747 in chronic pain (Le Bourdonnec et al., 2009) and ADL5859 in pain and depression (Bourdonnec et al., 2008).

The highest densities of DOPr in rat brain are located within the cortex, amygdala, striatum, hippocampus and NAc (Lutz and Kieffer, 2013b), whereas high amounts of DOPr are found in olfactory bulb, hippocampus, amygdala basal ganglia and hypothalamus in human brain (Simonin et al., 1994) (see Figures 1.1 and 1.5 for the expression of DOPr in reward \& aversion and in pain pathway respectively). DOPrs have also been investigated for their role in the reward processes and in the development of addictive behaviours. DOPr agonists have shown to increase, whereas DOPr antagonists have shown to inhibit morphine reward and sensitization to morphine 
conditioned place preference (CPP) or heroin self-administration (Chefer and Shippenberg, 2009; Shippenberg et al., 2009; Suzuki et al., 1996). Chefer and Shippenberg (2009), were the first to demonstrate that the rewarding properties of morphine are decreased in DOPr KO mice. The study showed that morphine-induced context-dependent sensitization was enhanced and tolerance to the locomotor activating effects of morphine was attenuated when DOPr function was blocked pharmacologically or genetically. Furthermore, the conditioning of morphine reward was also attenuated. Together, these data indicate unique and contrasting roles of DOPr in the development and expression of long-term adaptations that occur in response to chronic MOPr agonist administration. The mechanisms mediating functional interactions between DOPr and MOPr are not clear. However, heterodimerization of MOPr and DOPr has been reported in cell expression systems (Gupta et al., 2006; Jordan and Devi, 1999; Law et al., 2005), and that chronic morphine exposure promotes an increase in DOPr cell-surface expression had also been obtained (Cahill et al., 2001; Morinville et al., 2003). A study addressed important interactions between DOPr and morphine tolerance, in this study blockade of DOPr with selective DOPr antagonist NTI (Naltrindole) and DOPr subtype 2antagonit naltriben significantly attenuated the development of morphine tolerance in rat model of chronic inflammatory pain (Beaudry et al., 2015).

Pharmacological blockade of DOPr decreased rewarding properties of psychostimulant like cocaine and methamphetamine (Belkaï et al., 2009; Le Merrer et al., 2011; Shippenberg et al., 2009). Moreover, brain specific infusion of DOPr agonists or antagonists also has shown to modify cocaine self-administration or cocaine seeking behaviours. In rats self-administering cocaine (1.5 $\mathrm{mg} / \mathrm{kg}$ ) under a progressive ratio schedule, microinjection of DOPr antagonist naltrindole- $5^{\prime}$ isothiocyanate (5'-NTII; 5nmol) into the NAc, VTA or amygdala significantly decreased cocaine self-administering in NAc, and increased cocaine-maintained responding in VTA, but had no effect in amygdala (Ward and Roberts, 2007). Whereas, administration of DOPr agonist ([D-Pen2,DPen5]-enkephalin (DPDPE); $300 \mathrm{ng} /$ side) into the NAc reinstated cocaine seeking behaviour in rats following extinction of cocaine self-administration (Simmons and Self, 2009). DOPr agonists have also shown to attenuate anxiety occurring in cocaine withdrawal-induced and showed anxiolytic effects in ethanol-withdrawn mice, although not effective in decreasing anxiety-like behaviours in naïve mice (Ambrose-Lanci et al., 2010; van Rijn et al., 2010). Within this line, DOPr agonists may be effective in preventing relapse by reducing emotional alterations that emerge during withdrawal periods. However, DOPr agonist induce seizures (Bilsky et al., 1995; Comer et al., 1993; Jutkiewicz et al., 2006; Lutz and Kieffer, 2013a). 
Combining DOPr ligands with KOPr ligand activity may be helpful in developing a medication with a non-addictive analgesic property. KOPr ligands in combination with other opioid receptor (MOPr and DOPr) ligands are discussed below (see Table 1.2 also).

\subsubsection{KOPr/DOPr ligands}

Previous literature proposed KOPr/DOPr interactions for the treatment of depression. Simultaneous administration of ADL5859, a DOPr agonist and LY2444296, a KOPr antagonist resulted in synergistic anti-depressant-like effects in FST in mice (Huang et al., 2016). It has been suggested that activation of DOPr may have the potential to reverse stress-induced depressive behaviours that result from KOPr activation. Few compounds possessing dual KOPr/DOPr activity have been reported previously. These include KDA-16 (obtained from KOPr pharmacophore,2(3,4-dichlorophenyl)- $N$-methyl- $N$-[(1S)-1-phenyl-2-(1-pyrrolidinyl) ethyl] acetamide hydrochloride - ICl-199,441), which showed spinal anti-nociception via selective activation of KOPr-DOPr heteromers in mice (Tang et al., 2010). In contrast, KDN-21, which links KOPr antagonist, 5'- GNTI to DOPr antagonist, NTI, did not show anti-nociceptive effects in mice (Bhushan et al., 2004). However, it showed heterodimeric KOPr/DOPr interactions in HEK-293 cells expressing rat DOPr and mouse KOPr (Xie et al., 2005) and in mouse spinal cord (Bhushan et al., 2004). KDAN-18, which links KOPr agonist, ICl-199,441 and DOPr antagonist, NTI, showed antinociceptive effects in tail-withdrawal assays in male mice (Daniels et al., 2005) (see Table 1.2). Although these previous studies assessed the anti-nociceptive effects of dual KOPr/DOPr ligands, there no studies reporting the effects on reward. To address this gap, in this study we evaluated the anti-cocaine effects of our novel dual KOPr/DOPr agonist, MP1104 in male Sprague-Dawley rats. 


\subsubsection{MOPr/KOPr ligands}

Studies reported that mixed MOPr/DOPr ligands are encouraged for the treatment of addiction to cocaine and other drugs of abuse (Greedy et al., 2013), and that a KOPr antagonist/MOPr agonist could be beneficial for preventing relapse to drugs of abuse (Toll et al., 2013). It has been suggested that the euphoric effects associated with weak MOPr agonist may be able to balance dysphoric effects associated with KOPr agonists. Thus, mixed MOPr/KOPr agonist offer potential advantage over selective KOPr agonist for the treatment of drug abuse. The examples of this mixed agonism include nalbuphine, which has been shown to attenuate cocaine abuse related effects in humans (Mello et al., 2005). Butorphan (MCL-101) was shown to decrease the rewarding effects of cocaine in intracranial self-stimulation studies in rats (Provencher et al., 2013) and dose-dependently decreased cocaine self-administration with minimal side effects in rhesus monkeys (Bowen et al., 2003b), like (salivation) compared to selective KOPr agonist that were reported to produce sedation, salivation and emesis. 3-Amino-thiazolo[5,4-b]-Ncyclopropylmethylmorphinan hydrochloride (ATPM), and its analogue ET-ATPM, with mixed KOPr and MOPr agonist/antagonist properties showed anti-nociceptive effects and also inhibited morphine-induced anti-nociceptive tolerance in mice (Wang et al., 2009; Sun et al., 2010). Similarly BNAP, a mixed KOPr agonist/MOPr antagonist ligand showed anti-nociceptive effects in mice (Williams et al., 2016) (see Table 1.2).

\subsubsection{Other mixed ligands}

Other mixed ligands with less or no KOPr activity are discussed here. It has been reported in the literature that the co-administration of DOPr agonist with a MOPr agonist reduces the development of tolerance and dependence on MOPr agonists without attenuating MOPrmediated analgesia, and co-administration of DOPr antagonist with a MOPr agonist also decreases the adverse effects typically associated with MOPr agonists (Li et al., 2012; Lowery et al., 2011). Hence, mixed MOPr/DOPr ligands have been explored previously (Dietis et al., 2009; Horan et al., 1993; Martin et al., 2000; Zielińska et al., 2016) (see Table 1.2 for details).

Studies reported that co-administration of a DOPr agonist with a MOPr agonists causes reduced tolerance or dependence to MOPr agonists without attenuating the development of MOPr mediated analgesia (Lowery et al., 2011; Rozenfeld et al., 2007). It has also been shown that the efficacy and anti-nociception potency of MOPr agonists is enhanced up on co-administering small 
doses of DOPr agonists (Heyman et al., 1989; Horan, Tallarida, et al., 1992). A study showed that co-administration of DOPr antagonist, $5^{\prime}$-NTII with an addictive MOPr agonist such as heroin reduced the self-administration of heroin in rats (Martin et al., 2000). This data indicates that coadministration of DOPr antagonist with a traditional MOPr agonist may prevent adverse effects and minimise the abuse potential associated with chronic MOPr use, thus providing safer alternative to traditional opioid analgesics. Mixed ligands with MOPr/DOPr agonists activity include like Biphalin and MMP-2200 (Lowery et al., 2011; Sobczak et al., 2014). Whereas, mixed MOPr agonist/DOPr antagonist acting ligands include MDAN-21, UFP-505, DIPP $\Psi \mathrm{NH}_{2}$ and VRP26, which have shown to produce anti-nociceptive effects with reduced side effects in rats and mice (Schiller et al., 1999; Aceto et al., 2012; Dietis et al., 2012; Mosberg et al., 2014) (see Table 1.2 for details).

Cebranopadol (also known as GRT 60005) and BU08028 are agonists at MOPr, DOPr, KOPr and NOPr and demonstrated anti-nociceptive in rats and rhesus monkeys respectively with favourable side effect profile (Linz et al., 2014; Ding et al., 2016). Cebranopadol is currently in Phase III clinical trials for several indications including cancer pain (Lambert et al., 2014). KGNOP1, a MOPr agonist/NOPr antagonist was recently reported a candidate for dual treatment of nociception and neuropathic pain as investigated in male Sprague-Dawley rats using hotplate test and cold hyperalgesia model of neuropathic pain induced by chronic constriction injury of the sciatic nerve (Lagard et al., 2017). Whereas, SR1635 and SR14150 are agonists at MOPr and NOPr with potent anti-nociceptive effects in mice (Khroyan et al., 2007, 2011) (see Table 1.2 for details).

All these studies show that mixed opioid receptor ligands have potential therapeutic effects with reduced side effects compared to pure MOPr, KOPr or DOPr agonists. 
Table 1.2: Mixed opioid receptor ligands and their effects

\begin{tabular}{|c|c|c|c|c|c|c|}
\hline & \multirow[t]{2}{*}{ Compound } & \multicolumn{4}{|c|}{ Effects } & \multirow[t]{2}{*}{ Subjects } \\
\hline & & Anti-cocaine & Anti-nociceptive & Side effects & Other effects & \\
\hline \multirow[t]{3}{*}{ KOPr/MOPr agonists } & Nalbuphine & $\begin{array}{c} \\
(5 \mathrm{mg} / 70 \mathrm{~kg}, \text { i.v. })^{(1)}\end{array}$ & $\begin{array}{c}\checkmark \\
(0.15 \mathrm{mg} / \mathrm{kg})^{(2)}\end{array}$ & - & - & $\underset{(1,2)}{\operatorname{Humans} \text { (males) }}$ \\
\hline & Butorphan (MCL-101) & $\begin{array}{c}\checkmark \\
\left(4 \mathrm{mg} / \mathrm{kg}, \text { i.v. }{ }^{(3)}\right. \\
0.0032-0.032 \\
\mathrm{mg} / \mathrm{kg}, \text { i.v. }^{(4)}\end{array}$ & - & - & - & $\begin{array}{c}\text { Male Sprague- } \\
\text { Dawley rats }{ }^{(3)} \& \\
\text { rhesus monkeys }{ }^{(4)}\end{array}$ \\
\hline & $\begin{array}{l}\text { Endomorphin II } \\
\text { analogue }\end{array}$ & - & $\begin{array}{c}\checkmark \\
\left(E D_{50}=0.714 \mu \mathrm{g} / \mathrm{animal},\right. \\
\text { i.c.v. \& } 3 \text { \& } 10 \mathrm{mg} / \mathrm{kg} \text {, i.p. }^{(5)}\end{array}$ & - & - & $\begin{array}{l}\text { Male albino } \\
\text { BALB/c mice }\end{array}$ \\
\hline \multirow[t]{2}{*}{$\begin{array}{l}\text { KOPragonist/MOPr } \\
\text { agonist/antagonist }\end{array}$} & ATPM & - & $\begin{array}{c}\checkmark \\
\left(0.4 \mathrm{mg} / \mathrm{kg} \text {, s.c.) }{ }^{(6)}\right.\end{array}$ & $\begin{array}{c}\downarrow \\
\downarrow \\
\text { morphine } \\
\text { tolerance }\end{array}$ & - & $\begin{array}{l}\text { Kunming strain } \\
\text { mice }\end{array}$ \\
\hline & ET-ATPM & - & $\begin{array}{c}\checkmark \checkmark \\
\left(E D_{50}=2.68 \mathrm{mg} / \mathrm{kg} \text {, s.c. }\right)^{(7)}\end{array}$ & - & $\begin{array}{c}\downarrow \\
\text { morphine- } \\
\text { induced } \\
\text { behavioural } \\
\text { sensitisation (1.5 } \\
\text { and } 3 \mathrm{mg} / \mathrm{kg} \text {, s.c.) }\end{array}$ & $\begin{array}{l}\text { Male Kunming } \\
\text { strain mice }\end{array}$ \\
\hline $\begin{array}{l}\text { KOPragonist/MOPr } \\
\text { antagonist }\end{array}$ & BNAP & - & $\begin{array}{c}\checkmark \\
(10 \mathrm{mg} / \mathrm{kg}, \text { s.c. })^{(8)}\end{array}$ & - & - & $\begin{array}{l}\text { Male Swiss } \\
\text { webster mice }\end{array}$ \\
\hline \multirow[t]{2}{*}{$\begin{array}{l}\text { KOPr/DOPr mixed } \\
\text { ligands }\end{array}$} & KDAN-18 & - & $\begin{array}{c}\checkmark \checkmark \\
\left(\mathrm{ED}_{50}=0.19 \mathrm{nmol} / \mathrm{mouse}\right. \\
{\text { i.c.v. })^{(9)}}^{(9)}\end{array}$ & - & - & Male CD1 mice \\
\hline & KDA-16 & - & \begin{tabular}{l}
\multicolumn{1}{c}{$\checkmark$} \\
$\left(E D_{50}=0.58 \mathrm{nmol} /\right.$ mouse, \\
i.c.v; $E D_{50}=0.0045$ \\
nmol $/$ mouse i.t. $)^{(10)}$
\end{tabular} & - & - & Male CD1 mice \\
\hline
\end{tabular}




\begin{tabular}{|c|c|c|c|c|c|c|}
\hline \multirow[t]{2}{*}{ MOPr/DOPr agonists } & Biphalin & - & $\begin{array}{c}\checkmark \\
(5 \mathrm{mg} / \mathrm{kg} \text {, i.p. })^{(11)}\end{array}$ & - & $\begin{array}{c}\text { Anti- } \\
\text { inflammatory (5 } \\
\mathrm{mg} / \mathrm{kg} \text {, twice } \\
\text { daily,i.p.) })^{(11)} \\
\text { Anti-transit (5 } \\
\mathrm{mg} / \mathrm{kg} \text {, i.p.) })^{(12)}\end{array}$ & $\begin{array}{c}\text { Male albino } \\
\text { BALB/c mice }{ }^{(11,12)}\end{array}$ \\
\hline & MMP-2200 & - & $\begin{array}{c}\checkmark \\
\left(E D_{50}=0.049 \mathrm{nmol}, \text { i.t. }\right)^{(13)}\end{array}$ & $\begin{array}{c}\downarrow \\
\text { morphine } \\
\text { tolerance, } \\
\text { dependence \& } \\
\text { locomotor }^{(13)} \\
\text { stimulation }^{(13)}\end{array}$ & $\begin{array}{c}\text { Anti-allodynic } \\
(0.032-3.2 \mathrm{mg} / \mathrm{kg} \text {, } \\
\text { i.m. })^{(14)}\end{array}$ & $\begin{array}{l}\text { Male ICR mice }{ }^{(13)} \\
\text { Rhesus monkeys } \\
(14)\end{array}$ \\
\hline \multirow[t]{2}{*}{$\begin{array}{l}\text { MOPragonist/DOPr } \\
\text { antagonist }\end{array}$} & MDAN-21 & - & - & - & $\begin{array}{l}\text { Anti-allodynic } \\
(0.0032-0.032 \\
\mathrm{mg} / \mathrm{kg}, \text { i.m. }{ }^{(15)}\end{array}$ & Rhesus monkeys \\
\hline & VRP26 & - & $\begin{array}{c}\checkmark \\
(10 \mathrm{mg} / \mathrm{kg}, \text { i.p. })^{(18)}\end{array}$ & $\begin{array}{c}\downarrow \\
\text { fentanyl } \\
\text { tolerance, } \\
\text { dependence } \\
(10 \mathrm{mg} / \mathrm{kg}, \text { i.v. }) \\
(19) \\
\downarrow \\
\text { fentanyl CPP } \\
(1,3.2,10 \text { or } 32 \\
\text { mg/kg, i.p.) }{ }^{(19)}\end{array}$ & - & $\begin{array}{c}\text { Mice }{ }^{(18)} \\
\text { Male and female } \\
\text { C57BL/6J mice }{ }^{(19)}\end{array}$ \\
\hline
\end{tabular}




\begin{tabular}{|c|c|c|c|c|c|c|}
\hline & \multirow[t]{2}{*}{ Compound } & \multicolumn{4}{|c|}{ Effects } & \multirow[t]{2}{*}{ Subjects } \\
\hline & & Anti-cocaine & Anti-nociceptive & Side effects & Other effects & \\
\hline \multirow[t]{5}{*}{$\begin{array}{l}\text { Other mixed opioid } \\
\text { receptor ligand }\end{array}$} & $\begin{array}{l}\text { Cebranopadol (GRT } \\
\text { 60005) - MOPr, DOPr, } \\
\text { KOPr and NOPr } \\
\text { agonist }\end{array}$ & - & $\begin{array}{c}\checkmark \\
\left(E D_{50}=5.6 \mu \mathrm{g} / \mathrm{kg}, \text { i.v. \& }\right. \\
\left.\mathrm{ED}_{50}=21.5 \mu \mathrm{g} / \mathrm{kg}, \text { p.o. }\right)^{(20)}\end{array}$ & $\begin{array}{l}\quad \downarrow \\
\text { Motor } \\
\text { coordination and } \\
\text { respiration }\end{array}$ & - & $\begin{array}{l}\text { Sprague-Dawley } \\
\text { rats }\end{array}$ \\
\hline & $\begin{array}{l}\text { BU08028 - MOPr, } \\
\text { DOPr, KOPr and NOPr } \\
\text { agonist }\end{array}$ & - & $\begin{array}{c}\checkmark \\
(0.001-0.01 \mathrm{mg} / \mathrm{kg}, \text { s.c. })^{(21)}\end{array}$ & $\begin{array}{c}\downarrow \\
\text { Respiratory } \\
\text { depression }\end{array}$ & $\begin{array}{l}\text { Less rewarding } \\
\text { than cocaine, } \\
\text { remifentanil and } \\
\text { buprenorphine } \\
\end{array}$ & Rhesus monkeys \\
\hline & $\begin{array}{l}\text { KGNOP1 - MOPr } \\
\text { agonist/NOPr } \\
\text { antagonist }\end{array}$ & - & $\begin{array}{c}\checkmark \\
\left(E D_{50}=0.0004 \mu \mathrm{mol} / \mathrm{kg},\right. \\
\text { i.v.; } 0.41 \mu \mathrm{mol} / \mathrm{kg} \text {, i.v.) }{ }^{(22)}\end{array}$ & $\begin{array}{c}\downarrow \\
\text { Respiratory } \\
\text { depression } \\
\text { compared to } \\
\text { morphine and } \\
\text { tramadol }\end{array}$ & - & $\begin{array}{l}\text { Male Sprague- } \\
\text { Dawley rats }\end{array}$ \\
\hline & $\begin{array}{l}\text { SR16435 - } \\
\text { MOPr/NOPr agonist }\end{array}$ & - & $\begin{array}{c}\checkmark \\
(10 \mathrm{mg} / \mathrm{kg} \text {, s.c. })^{(23)}\end{array}$ & - & $\begin{array}{l}\text { Anti-allodynic } \\
\left(0.1-3 \mu g^{2} \text { i.t. }\right)^{(24)} \\
\text { Anti-hyperalgesic } \\
(0.03-1 \mu g, \text { i.t. })^{(24)} \\
\end{array}$ & $\begin{array}{l}\text { Male ICR mice } \\
\text { 24) }\end{array}$ \\
\hline & $\begin{array}{l}\text { SR14150 (AT-200) - } \\
\text { MOPr/NOPr agonist }\end{array}$ & - & $\begin{array}{c}\checkmark \\
\text { (3 \& } 10 \mathrm{mg} / \mathrm{kg} \text {, s.c. }{ }^{(25)} \& 3- \\
15 \mathrm{mg} / \mathrm{kg} \text {, s.c.) }{ }^{(26)}\end{array}$ & $\begin{array}{l}\text { No morphine- } \\
\text { induced CPP (26) }\end{array}$ & $\begin{array}{l}\text { Anti-allodynic } \\
(10 \mathrm{mg} / \mathrm{kg} \text {, s.c. })^{(25)}\end{array}$ & $\begin{array}{l}\text { Male ICR mice } \\
\text { 26) }\end{array}$ \\
\hline
\end{tabular}

$\checkmark=$ presence of effect, $\downarrow=$ attenuation, $-=$ study not found, CPP = Conditioned place preference. 
1) Mello et al. (2005)

2) Gal et al. (1982)

3) Provencher et al. (2013)

4) Bowen et al. (2003a)

5) Perlikowska et al. (2016)

6) Wang et al. (2009)

7) Sun et al. (2010)

8) Williams et al. (2016)

9) Daniels et al. (2005)
10) Tang et al. (2010)

11) Sobczak et al. (2014)

12) Zielińska et al. (2016)

13) Lowery et al. (2011)

14) Do Carmo et al. (2009)

15) Aceto et al. (2012)

16) Dietis (2012)

17) Schiller et al. (1999)

18) Mosberg et al. (2014)
19) Anand et al. (2016)

20) Linz et al. (2014)

21) Ding et al. (2016)

22) Lagard et al. (2017)

23) Khroyan et al. (2007)

24) Sukhtankar et al. (2013)

25) Khroyan et al. (2011)

26) Toll et al. (2009) 


\subsection{MP1104, a novel mixed opioid receptor agonist}

MP1104,17-Cyclopropylmethyl-3-hydroxy-4,5 $\alpha$-epoxy-7,8-en-6- $\beta$-[(3'-iodo)benzamido]-

morphinan (see Figure 1.6), is an analogue of $3^{\prime}$-lodobenzoyl naltrexamine - a MOPr agonist belonging to $6 \beta$-amido $14-\mathrm{OH}$-epoxymorphinan class. It was synthesised by medicinal chemist $\mathrm{Dr}$ Susruta Majumdar and his team members from Dr Gavril Pasternak laboratory (Memorial Sloan Kettering cancer centre, New York City, USA). MP1104 is a novel mixed opioid agonist that has high affinity for the KOPr in [ ${ }^{125}$ I]BNtxA competitive binding assays, with KOPr showing 3- and 13fold higher binding affinity compared to that of MOPr and DOPr respectively (Varadi et al., 2015) (see Table 1.4). Recently, the crystal structure of human KOPr in complex with MP1104 and an active-state-stabilizing nanobody has been explored to provide molecular insights into KOPr structure and function (Che et al., 2018). In functional $\left[{ }^{35} \mathrm{~S}\right] \mathrm{GTP} \gamma \mathrm{S}$ binding assays in Chinese hamster ovary $(\mathrm{CHO})$ cells expressing cloned mouse opioid receptors, MP1104 showed 8- and 15fold greater potency at KOPr compared to MOPr and DOPr, respectively (Varadi et al., 2015) (see Table 1.4).

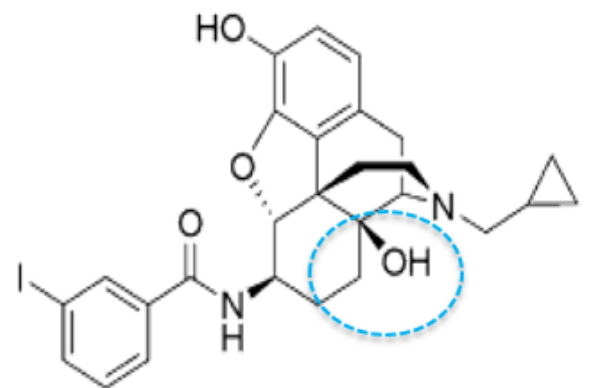

3-lodobenzoyl naltrexamine

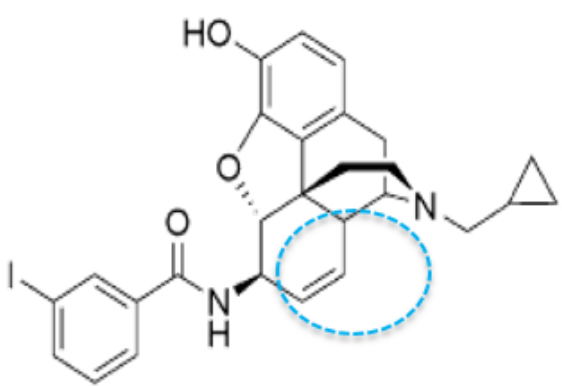

MP1104

\section{Figure 1.6: Chemical structures of MP1104 and 3-lodobenzoyl naltrexamine}

MP1104 was obtained by structural modifications of its parent compound, 3-lodobenzoyl naltrexamine by removal of C-14 hydroxyl functional group and addition of a double bond at C 78 Varadi et al. (2015). 
Table 1.3: Binding affinity, efficacy and potency of MP1104

\begin{tabular}{|c|c|c|c|}
\hline \multicolumn{4}{|l|}{ Binding affinity } \\
\hline Chemical compound & $\mathrm{MOPr}$ & $\mathrm{KOPr}$ & $D O P r$ \\
\hline MP1104 & $\mathrm{K}_{\mathrm{i}}=0.021 \pm 0.0034$ & $\mathrm{~K}_{\mathrm{i}}=0.0064 \pm 0.002$ & $K_{i}=0.08 \pm 0.019$ \\
\hline Morphine & $\mathrm{K}_{\mathrm{i}}=4.6 \pm 1.81^{(2)}$ & - & - \\
\hline DAMGO & $K_{i}=3.34 \pm 0.43^{(2)}$ & - & - \\
\hline U50,488 & - & $\mathrm{K}_{\mathrm{i}}=0.73 \pm 0.32^{(2)}$ & - \\
\hline DPDPE & - & - & $K_{i}=1.39 \pm 0.67^{(2)}$ \\
\hline \multicolumn{4}{|l|}{ Potency and efficacy } \\
\hline Chemical compound & $\mathrm{MOPr}$ & KOPr & DOPr \\
\hline MP1104 & $\begin{array}{l}\mathrm{EC}_{50}=0.21 \pm 0.03 \\
\mathrm{E}_{\max }=103 \pm 2.5\end{array}$ & $\begin{array}{l}E C_{50}=0.027 \pm 0.002 \\
E_{\max }=104 \pm 2.3\end{array}$ & $\begin{array}{l}E C_{50}=0.41 \pm 0.11 \\
E_{\max }=88 \pm 0.38\end{array}$ \\
\hline DAMGO & $\mathrm{EC}_{50}=19 \pm 7.0$ & n.d. & n.d. \\
\hline U50,488 & n.d. & $\mathrm{EC}_{50}=17 \pm 6.1$ & n.d. \\
\hline DPDPE & n.d. & n.d. & $\mathrm{EC}_{50}=10 \pm 2.2$ \\
\hline
\end{tabular}

- = study not found, n.d. = not determined.

$\begin{array}{ll}\text { 1) (Varadi et al., 2015) } & \text { 2) (Majumdar et al., 2011) }\end{array}$

Binding affinity $\left(\mathrm{K}_{\mathrm{i}}\right)$ at MOPr, KOPr and DOPr using [ $\left.{ }^{125} \mathrm{I}\right] \mathrm{BNtxA}(0.1 \mathrm{nM})$ competition binding assays performed in membranes prepared from Chinese hamster ovary $(\mathrm{CHO})$ cells expressing mouse MOPr, KOPr or DOPr ${ }^{(1)}$. Potency, efficacy at the MOPr, KOPr, DOPr using $\left[{ }^{35} \mathrm{~S}\right] \mathrm{GTP} \gamma \mathrm{S}$ functional assay using membranes prepared in $\mathrm{CHO}$ cells expressing mouse MOPr, $\mathrm{KOPr}$ or $\mathrm{DOPr}^{(1)}$. Values presented as the mean or mean $\pm \mathrm{SEM} ; \mathrm{K}_{\mathrm{i}}$ and median effective concentration $\left(\mathrm{EC}_{50}\right)$ in $\mathrm{nM}$, maximum response $\left(E_{\max }\right)$ in \%. DAMGO = [D-Ala2,N-MePhe4,Gly-ol]-enkephalin; DPDPE = [DPen2,D-Pen5]-enkephalin. 
A previous study by Varadi et al. (2015) has investigated the properties of MP1104 in behavioural models in mice in assays of anti-nociceptive pain, reward (CPP) and aversion (CPA). In the warmwater tail withdrawal assay in C57BL/6J mice, MP1104 was shown to have high potency $\left(E_{50}=\right.$ $0.33 \mathrm{mg} / \mathrm{kg}$ ) compared to morphine. Interestingly, although MP1104 showed full agonism at MOPr in in vitro assays (Table 1.3), the MOPr did not mediate the anti-nociceptive effects. Analgesia was found to be insensitive to the MOPr antagonist $\beta$-funaltrexamine ( $\beta$-FNA), and MOPr KO mice and was found to be KOPr and DOPr dependent (Varadi et al., 2015). In an inflammatory pain model, administration of MP1104 (1 mg/kg, s.c.) blocked the formalinmediated pain behaviours in mice (Dr Susruta Majumdar, Memorial Sloan Kettering cancer centre, personal communication).

The clinical applicability of mixed KOPr/ DOPr agonists has been thought to be limited because DOPr agonists induce seizures (Bilsky et al., 1995; Comer et al., 1993; Jutkiewicz et al., 2006; Lutz and Kieffer, 2013a). However, MP1104 did not produce seizures even at doses 30 times its antinociceptive $E_{50}$ value (Varadi et al., 2015). These findings highlight that mixed opioid agonists have properties that cannot be attributed to their ability to activate each receptor. MP1104 also has unique behavioural effects. For example, MP1104 was found to be neither aversive nor rewarding at doses of $1 \mathrm{mg} / \mathrm{kg}$ (Varadi et al., 2015). This is surprising, it suggests that MOPr and DOPr activation is not sufficient to produce their typical rewarding effects. Moreover, MP1104 prevented significant place preference produced by cocaine in mice (Varadi et al., 2015). This suggests that MP1104 possesses anti-addiction effects. This led us to hypothesise that KOPr activation may reduce the rewarding effects of MOPr and DOPr agonism, hence we evaluated the anti-cocaine effects of MP1104 using drug self-administration model, moreover MP1104 (1 $\mathrm{mg} / \mathrm{kg}$, i.p.) was also shown to block alcohol CPP in mice (Dr Susruta Majumdar, personal communication). 


\subsection{Overall aims and hypotheses}

With no current FDA approved treatments for psychostimulant abuse and with the rise in the abuse of prescription opioids, due to lack of available effective pain medications, there is an urgent need for the development of non-addictive pain therapies and effective treatments to combat drug abuse.

This research aimed to evaluate the anti-cocaine and analgesic effects of a novel mixed opioid receptor agonist, MP1104, with the hypothesis that mixed opioid receptor agonists have a better therapeutic profile compared to selective MOPr, KOPr or DOPr agonists.

To evaluate the therapeutic potential of MP1104 we used animal models of drug addiction (drug self-administration); nociceptive pain (warm-water tail withdrawal assay); neuropathic pain (paclitaxel-induced neuropathic pain model) and behavioural measures of side effects (sedation, aversion, anxiety and depression measured using spontaneous locomotor activity, CPA, EPM and FST assays respectively).

\section{Specific aims:}

1. To evaluate the effect of acute administration of MP1104 on drug seeking and drug taking behaviours in rats trained to self-administer cocaine.

2. To investigate the anti-nociceptive effects of MP1104 using warm-water tail withdrawal assay in mice and rats.

3. Test the efficacy of MP1104 on paclitaxel-induced neuropathic pain using an acute doseresponse model for mechanical and cold allodynia. Further, assess MP1104 effects in a chronic model of paclitaxel-induced neuropathic pain in mice.

4. To evaluate the side effects like sedation, anxiety, aversion and pro-depressive using spontaneous locomotor activity, elevated plus maze, conditioned place aversion and forced swim tests respectively. 


\section{Chapter 2: Materials and methods}

\subsection{Materials}

Carprofen (Carprieve; $50 \mathrm{mg} / \mathrm{mL}$ ), heparin sodium (5,000 unit/mL), vetadine, ketamine (100 $\mathrm{mg} / \mathrm{mL}$ ), lacrilube eye ointment, penicillin $\mathrm{G}$ sodium $(1,000,000 \mathrm{IU})$, pentobarbital $(500 \mathrm{mg} / \mathrm{mL})$, sodium lactate (Hartman's solution), supramid suture wire (\#F1184040, Braun), terramycin powder, vetadine (1.6\% iodine, $\mathrm{w} / \mathrm{v}$ ) and xylazine $(20 \mathrm{mg} / \mathrm{mL})$ were obtained from Provet NZ Pyt Ltd (Palmerston North, NZ). For cocaine self-administration experiment, Cocaine- $\mathrm{HCl}$ was obtained from BDH Ltd., Wellington, NZ. Polysorbate 80 (Tween-80), Dopamine-HCl, U50,488 and cremophor EL were purchased from Sigma-Aldrich (Auckland, NZ). Ethanol, dimethyl sulfoxide (DMSO) and acetone (OPTIMA grade ACS, Fisher Scientific, UK) were obtained from Thermofisher Scientific NZ Ltd., (Auckland, NZ). KOPr antagonist nor-BNI and Yohimbine were purchased from In Vitro Technologies Ltd., Auckland, NZ. MP1104 and NTI, both with about 98\% purity (Varadi et al., 2015) were generous gifts from Dr Susruta Majumdar. Paclitaxel (300 mg in 50 mL; Sandoz Pty Ltd., NSW, Australia) and morphine sulphate (10 mg/mL in saline, Hospira NZ Ltd.) were purchased from the Capital and Coast District Health Board Pharmacy (Wellington, NZ).

Equipment for surgery: Tygon tubing (\#C-P06419-01; internal diameter: 0.020 inches, outer diameter: 0.060 inches, 100ft, Cole Parmer) and silastic tubing (\#C-P96115-02; internal diameter: $0.5 \mathrm{~mm}$, outer diameter: $0.9 \mathrm{~mm}, 50 \mathrm{ft}$, Cole Parmer) were purchased from Thermofisher Scientific NZ Ltd., (Auckland, NZ). The jeweller's screws were purchased from Eyeline Optical (Wellington, NZ) and large screws from Coastal Fasteners (Wellington, NZ). Dental acrylic (Ostron powder and liquid) was bought from Henry Schein Shalfoon (Auckland, NZ). Superglue (Bostik) and Silicon (Selleys) were purchased from Bunnings warehouse (Wellington, NZ). The microfilters (\#16534-K; $0.2 \mu \mathrm{m} \times 28 \mathrm{~mm}$ ) were purchased from MicroNZ. All needles and syringes were obtained from Becton Dickinson Pty (Auckland, NZ), cotton buds from Johnson \& Johnson (Auckland, NZ) and gauze swabs from Global science (Auckland, NZ).

All animal housing equipment was purchased from Able Scientific (Perth, Australia). Animal feed (Diet 86) was purchased from Sharpes Stock Feeds (Carterton, NZ) and the bedding (Aspen fine bedding) for animals was acquired from Fort Richard Laboratories Ltd., (Auckland, NZ). 


\subsection{Drug preparation}

MP1104 (0.3, 0.6 and $1 \mathrm{mg} / \mathrm{kg}$; i.p.), DOPr antagonist NTI (15 mg/kg; s.c.), MOPr antagonist $\beta$-FNA (10 mg/kg, s.c.), U50,488 (10 mg/kg, i.p.), morphine (10 mg/kg; i.p.) and yohimbine $(2.5 \mathrm{mg} / \mathrm{kg}$, i.p.) were dissolved in a vehicle of DMSO, Tween 80 and milliQ water in a ratio 2:1:7. The KOPr antagonist nor-BNI (10 mg/kg, s.c.) was dissolved in physiological (0.9\%) saline. The pre-treatment times for MP1104, NTI, U50,488 and yohimbine were $10 \mathrm{~min}, 15 \mathrm{~min}, 10 \mathrm{~min}$ and $30 \mathrm{~min}$ respectively. $\beta$-FNA and nor-BNI were injected via s.c. route of administration $24 \mathrm{~h}$ prior to testing. In rats, injections were given at $1 \mathrm{~mL} / \mathrm{kg}$ volumes.

Cocaine-prime was delivered via i.p. injections. Cocaine $(20 \mathrm{mg} / \mathrm{kg})$ was dissolved in sterile, filtered physiological saline. For cocaine delivered via i.v. infusions, it was dissolved in sterile physiological saline containing heparin sodium ( 3 unit/mL). $0.2 \mathrm{~mL}$ of penicillin/heparin solution (30 unit $/ \mathrm{mL}$ ) was flushed daily through the rat catheters to prevent infection and clot formation. Again, filtered sterile saline was used to dilute carprofen $(5 \mathrm{mg} / \mathrm{mL})$ and pentobarbital $(50 \mathrm{mg} / \mathrm{mL})$ to their respective working solutions.

In mice, MP1104 and morphine were dissolved in DMSO:Tween-80:saline in a ratio of 2:1:7 and delivered via s.c. or i.p. route of administration for anti-nociceptive studies. Injections in mice were given at a volume of $10 \mathrm{~mL} / \mathrm{kg}$ (unless otherwise stated). Paclitaxel ( $4 \mathrm{mg} / \mathrm{kg}$, i.p.) was diluted in sterile saline. For chronic administration experiment, drugs were made up in stock solutions and used within a maximum of 7 days prior to the preparation of fresh solution. The stock solution consisted of Tween-80 and DMSO portion of the vehicle solution, and each day to an aliquot of this stock physiological saline was added to produce a final drug solution in a 2:1:7 vehicle containing DMSO:Twee-80:saline. The stock solutions were made up to the same volume and blinded to the experimenter. The drug treatments were revealed only after the completion of the experiment. All drugs and solutions were stored in the dark at $4^{\circ} \mathrm{C}$. 


\subsection{Animals}

Adult male Sprague-Dawley rats (Rattus norvegicus) weighing 250-400 g were used for all experiments and were housed individually for self-administration experiments. For all other studies, rats were socially housed three per cage (polycarbonate cages) within the vivarium at the School of Biological Sciences, Victoria University of Wellington, NZ. The temperature $\left(19-20^{\circ} \mathrm{C}\right)$ and humidity (55\%) in the animal housing facility was controlled with lights maintained on a 12-h light/12-h dark cycle with lights on at 07:00 h. Food and water were available ad libitum except during testing. All experimental procedures followed were approved and conducted in accordance with the guidelines of the Animal Ethics Committee of Victoria University of Wellington, NZ (approval numbers 22334; and 21480). For all behavioural tests, experimentation was carried out during the light cycle (unless specified) and rats were handled by the experimenter two to three days prior to testing in order to prevent handling stress. All efforts were made to minimize the number of animals used.

Adult male C57BL/6 mice (23-26 g) were used for warm-water tail withdrawal tests and chemotherapy-induced neuropathic pain assays. Mice were obtained from Malaghan Institute of Medical Research (Wellington, NZ) and were housed within the School of Biological Sciences, Victoria University of Wellington animal facility. Mice were habituated to the experimentation room for one hour before performing experiments or during handling days. All behavioural experiments were performed in the presence of white noise (67 dB). 


\subsection{Drug Self-Administration}

\subsubsection{Preparation of catheters}

To ensure complete drying of silicon coating during implanting in rats, catheters were prepared two days before surgery. The metal piece was made by blunting the ends of $22 \mathrm{G}$ needle using a hand-held rotating flat disc dremel (Dremel rotating tool kit, 3000, NZ). The metal piece was rinsed thoroughly in $70 \%$ ethanol to remove any particulate matter that may cause clotting. This metal piece was inserted into silastic tubing (internal diameter: $0.5 \mathrm{~mm}$, outer diameter: $0.9 \mathrm{~mm}$ ) and flushed with $70 \%$ ethanol several times to ensure no leakage along the length of the tubing. After allowing to dry, the catheter was coated with a smooth, thin layer of silicon at the attachment of metal piece-tubing and up to $3 \mathrm{~cm}$ length from the end of silastic tubing. A minimum of two coats were applied, giving each coat a day to dry before application of the next. Following application of the final coat, a small silicon ball that marked as an anchor between the catheter and the jugular vein was created and then allowed to dry overnight. The tubing end, which was to be inserted into the vein, was cut at an angle for smooth and convenient entry of the catheter into the jugular vein during surgery (see Figure 2.1).

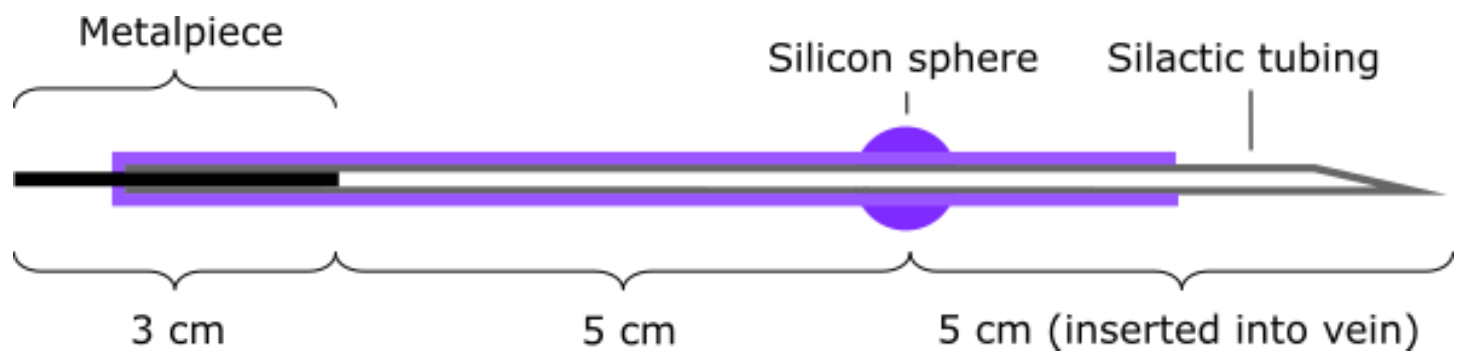

\section{Figure 2.1: Intrajugular catheter schematic diagram}

Intragular catheters were prepared two days prior to surgery to ensure that the two coats of applied silicon and silicon sphere were completely dry. Catheters were made up to $13 \mathrm{~cm}$ in total length, with the vein end to the silicon sphere measuring approximately $5 \mathrm{~cm}$. The vein end of the tubing was cut at an angle to allow for easier insertion into the jugular vein of the rat. 


\subsubsection{Surgery}

Rats weighing 300-350g were handled for two days before surgery. On the surgery day rats were deeply anaesthetised with an i.p. injection of ketamine $(90 \mathrm{mg} / \mathrm{kg})$ and xylazine $(9 \mathrm{mg} / \mathrm{kg})$. Rats were tested for the presence of a reflex by applying a strong pinching stimulus to the rear paw. The complete absence of a kicking reflex and loss of muscle tone indicated rats were completely anaesthetised and ready to undergo surgery. The head and chest area were shaved using animal clippers, and swabbed with vetadine and $70 \%$ ethanol to avoid any infections. Lacrilube eye ointment was applied on rat eyes to prevent dryness for the duration of surgery. An s.c. injection of carprofen $(5 \mathrm{mg} / \mathrm{kg})$, an analgesic, was administered on the right flank. A small incision was made on the right ventral side of the rat's chest area using a scalpel, in order to insert the catheter. The tissue was gently teased apart to locate the vein. The vein was then tied with a supramid suture wire at the rostral end. A small cut was made in the vein carefully and a silastic catheter was inserted into the vein. To ensure the catheter has been inserted correctly into the jugular vein, the intravenous line was verified by drawing back blood into the tygon tubing (served to flush the catheter) which was connected to the metal piece of the catheter to one end and the other end to a syringe filled with heparinised saline ( $3 \mathrm{unit} / \mathrm{mL}$ ). The exposed chest area was sealed with superglue. On the top of rat's head, a mid-sagittal incision was made and the distal end of the catheter was run subcutaneously to the exposed area of scalp from the chest region. The tissue on surface of the exposed scalp was gently scraped away using terramycin powder till the skull was visible. Into each quadrant of the skull four small holes were drilled using a manual hand drill (Evacut twist, Wellington, NZ) and small jeweller's screws embedded into each quadrant using a small flat head screwdriver. This was done to ensure proper adhesion of the headpiece to the skull. The metal piece was then bent at right angle and being held between the four screws was fixed in placed using superglue. Dentral acrylic paste was made by mixing Ostron powder and liquid to seal the exposed area of skull and to anchor the metal piece. A large screw was placed upside down on this quickly drying mixture and allowed to dry. In order to prevent clot formation a small piece of close-ended tubing was placed on the metal piece. Terramycin was dusted on the areas where the incisions were closed. The rats were then administered $12 \mathrm{~mL}(6 \mathrm{~mL}$ on each flank) of warm sodium lactate via s.c. injections to restore the lost fluids during surgery. Following this, rats were returned to their home cage with care. Post-surgery, rats were carefully monitored and a minimum of 5 days was required to recover. A further two day course of carprofen injections 
were given for pain relief. Animals were weighed daily and visually checked for any signs of discomfort.

\subsubsection{Testing of catheter patency}

For testing catheter patency, catheter was connected to a $1 \mathrm{~mL}$ syringe filled with penicillin/heparin solution ( $30 \mathrm{unit} / \mathrm{mL}$ ). About $0.1 \mathrm{~mL}$ volume of the solution was infused into the catheter and approximately $0.2 \mathrm{~mL}$ of the solution drawn back. The presence of blood indicated the patency of the catheter and rats labelled 'pass'. The rat was delivered further $0.2 \mathrm{~mL}$ of the penicillin/heparin solution and returned back to its home cage. If there was no visibility of blood, the next step was to infuse $0.15 \mathrm{~mL}$ of pentobarbital $(50 \mathrm{mg} / \mathrm{mL})$ and observe for immediate signs of anaesthesia, such as loss of muscle tone. If there was no loss of muscle tone, rats were declared to have failed the patency test. Then, rats were required to undergo another catheter implantation surgery on the left jugular vein the next day. Failure of the second catheter patency resulted in exclusion of rats from the study. If a block was identified due to the clot formation in the metal piece, the metal piece was replaced by inserting into the existing catheter and fixed to the headpiece under general anaesthetic conditions.

\subsubsection{Self-administration training}

Standard operant chambers (Med associates, ENV-001, St Albans, Vermont, USA) were used to train rats for cocaine self-administration. Each day before starting the session, the selfadministration chambers were prepared by flushing the tygon tubing to dispel any air bubbles and to ensure easy delivery of the solutions (Cocaine- $\mathrm{HCl}$ or heparinised saline) to the rats. The jugular catheters were flushed with penicillin/heparin solution before and after selfadministration sessions. A wire sheath (C313CS, Plastic One, Australia) surrounded the tygon tubing was connected to a swivel, allowing free movement of the rat while running the selfadministration experiment. The end of the tubing was attached to the metal piece of rat and the wire sheath was screwed on the rat's headpiece. The self-administration operant chambers consisted of two levers, an active lever, which was connected to a mechanical infusion pump and an inactive lever. Depression of the active lever delivered $0.1 \mathrm{~mL}$ infusion of cocaine- $\mathrm{HCl}(0.5$ $\mathrm{mg} / \mathrm{kg} /$ infusion) dissolved in heparinised saline for a period of $12 \mathrm{~s}$ accompanied with a light 
stimulus. The inactive lever presses were recorded, however, it had no programmed consequences. Rats were frequently checked to ensure that the tubing was intact with the catheter and making sure that the solutions are not running low from the syringes for the whole session.

The training began with rats allowed to self-administer cocaine- $\mathrm{HCl}$ on fixed ratio $(\mathrm{FR}) 1$ schedule of reinforcement, which indicates one active lever press delivers one infusion of cocaine- $\mathrm{HCl}$. When acquisition has been achieved i.e., rats self-administer at least 20 infusions during a $2 \mathrm{~h}$ session with $\geq 2: 1$ ratio of active:inactive lever press, rats were required to maintain this criteria for 3 consecutive days. Following this, rats were progressed to FR2, where two active lever presses delivered one infusion. Rats remained on this schedule until they learned to press at least 20 infusions ( 40 presses of active lever) in a $2 \mathrm{~h}$ session for 3 consecutive days, with the ratio of active:inactive lever $\geq 2: 1$. Following this, they then moved to FR5 schedule, where 5 lever presses delivered a single infusion. Rats had daily $2 \mathrm{~h}$ sessions 6 days/week (mon-sat) in the presence of white noise. A baseline level of active cocaine responding for each rat was calculated by taking the average infusions of FR5 schedule for a 3 consecutive days, with less than $20 \%$ variation in responses. All infusions and responses were recorded by Med Associates software (MED-PC IV, version 4.2). After establishment of stable baseline responding, rats were placed on extinction followed by cocaine-prime reinstatement testing.

\subsubsection{Cocaine-prime drug reinstatement tests}

The reinstatement test was conducted in rats stably responding to cocaine and had three phases (see Figure 2.2). Phase 1 consisted of $2 \mathrm{~h}$ cocaine self-administration on FR5. Rats were moved to phase 2 when responses were within $20 \%$ of baseline responses over 3 consecutive days. Phase 2 was the extinction phase, where cocaine was replaced with heparinised saline ( $3 \mathrm{unit} / \mathrm{mL}$ ) and the light cue removed. Animals remained on this schedule until lever responses dropped below 20 active lever press in a single daily session for 3-4 days. Phase 3, once extinction criteria were met, rats were subjected to a reinstatement test, where they were injected with either vehicle (DMSO:Tween-80:MilliQ water in a ratio of 2:1:7) or MP1104 (0.3 or $1 \mathrm{mg} / \mathrm{kg}$, i.p.) before receiving a priming injection of cocaine $(20 \mathrm{mg} / \mathrm{kg}$, i.p.), with the light cue restored and animals still receiving heparinised saline ( 3 unit/mL). In order to measure whether the reduced drug seeking effects were mediated via KOPr or DOPr, rats were pre-treated with $\mathrm{KOPr}$ (nor-BNI, $10 \mathrm{mg} / \mathrm{kg}$, 
s.c.) or DOPr (NTI, $15 \mathrm{mg} / \mathrm{kg}$, s.c.) antagonists. All treatments were administered using a withinsubject, Latin square design (Appendix C.1) except for nor-BNI, which was administered last due to its long-lasting effects (Endoh et al., 1992).

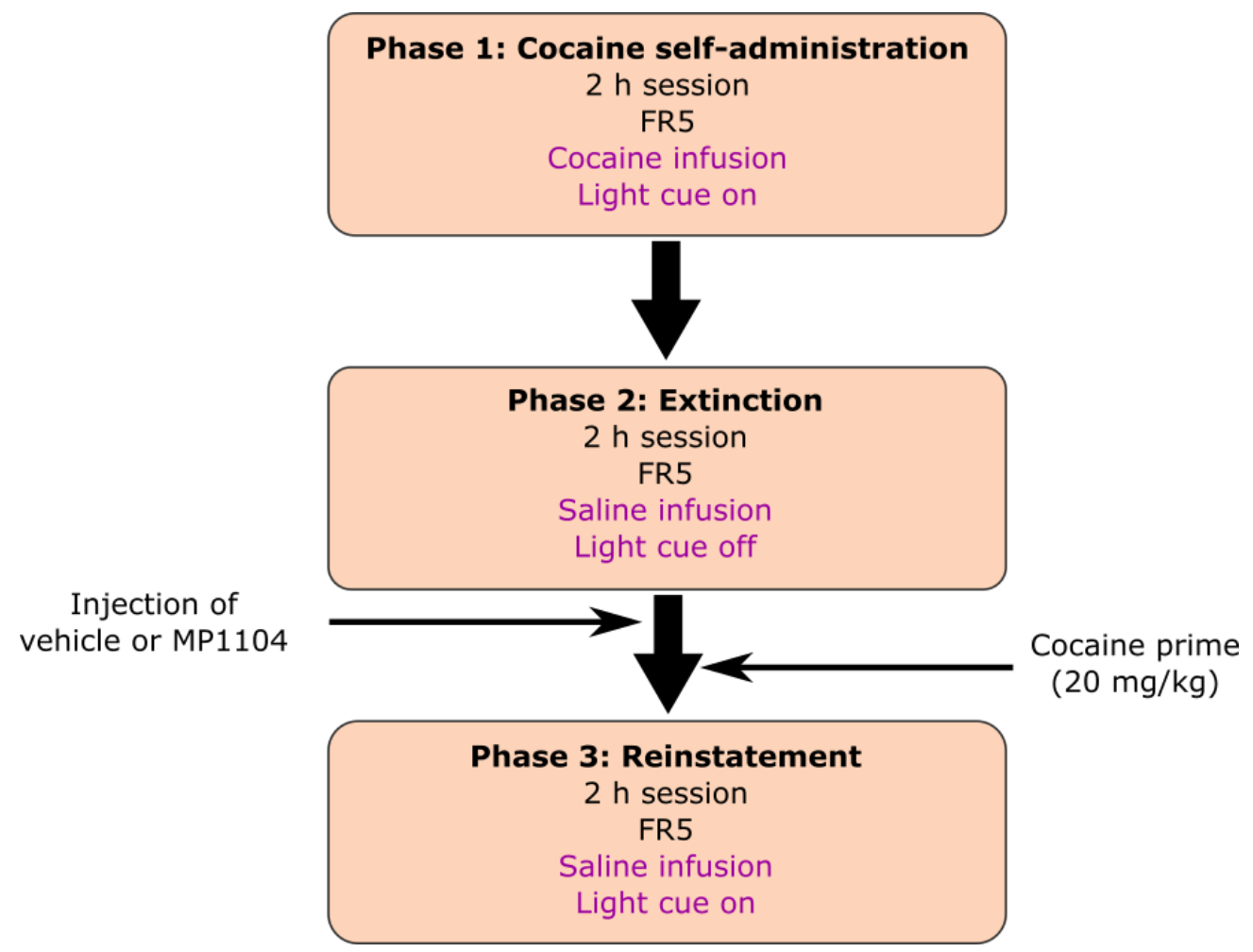

Figure 2.2: Cocaine-induced reinstatement of drug seeking procedure schematic diagram

Phase 1 included cocaine self-administration of rats, where an infusion of cocaine was delivered with the light cue on upon completion of the fixed ratio. Rats were progressed to Phase 2, an extinction phase, when the number of active lever presses during two consecutive sessions varied less than $20 \%$ of the baseline. In the extinction phase 2 cocaine was replaced with heparinised saline and removal of the light cue. Upon achieving extinction criteria rats were further progressed to phase 3 (reinstatement phase). In this phase, rats were injected with either vehicle or MP1104 before a priming injection of cocaine $(20 \mathrm{mg} / \mathrm{kg}$, i.p.) and immediately placed into the chamber after the cocaine injection and the number of active lever responses during this session were recorded. 


\subsubsection{Establishing a dose-response cocaine self-administration model}

To evaluate the effects of MP1104 on self-administration of various doses of cocaine we used the method of Song et al., (2012). The scripting of the programme followed manufacturer's instructions (MED-PC IV, Programmer's manual (Vermont, USA) and was carried out by Dr Anne Macaskill (Dept. of Psychology, VUW).

Briefly rats $(n=4)$ were placed on an FR2 schedule of reinforcement to determine baseline responding to cocaine doses $(0,0.15,0.5,1$ and $2 \mathrm{mg} / \mathrm{kg} /$ infusion). Following protocol testing the following doses of cocaine: $0.03,0.15,0.5,1$ and $2 \mathrm{mg} / \mathrm{kg} /$ infusion were found to produce a cocaine dose-response demonstrating an inverted $\mathrm{U}$-shape response with stable cocaine responding (less than $10 \%$ variability in number of active lever presses for 3 consecutive days).

\subsubsection{Dose-response cocaine self-administration tests}

In a single dose-response cocaine self-administration test, rats self-administered a full dose range of cocaine. Each comprised a 20 min component of self-administration at each dose, with a 20 min timeout (T.O) period (see Figure 2.3). During each T.O period, firstly, the experimenter disconnected the cocaine infusion tubing from the metal piece of animal (attachment which is placed on animals head through which cocaine infusion is delivered to the animal) to and sealed it with a small piece of close-ended tubing immediately, to prevent clotting. Then cocaine syringes with the next dose was replaced, ensuring there is continuous flow of fluid through the tubing prior to plugging it back to the metal piece. Performing this process within first 5 min of the T.O period was helpful, as rats had time to settle down following this interference by the experimenter. Before each daily cocaine self-administration test, a 30 min extinction period was included. The criteria for stable cocaine responding were a minimum of $10 \mathrm{mg} / \mathrm{kg}$ cocaine infusions per session, less than $10 \%$ variation in the total number of cocaine infusions for 3 consecutive days, and at least fivefold higher maximal response rates compared with those maintained during extinction (Song et al., 2012). Rats exhibited an inverted U-shaped function of cocaine dose (see Figure 3.3). As dose of cocaine increased, response rates increased, and then decreased gradually. Rats took about two weeks to be trained on a session of dose-response cocaine self-administration test, ensuring the fulfillment of the criteria. Once all criteria were achieved rats were given i.p. injections of either vehicle (DMSO:Tween-80:MilliQ water in a ratio 
of 2:1:7) or MP1104 (0.3 or $0.6 \mathrm{mg} / \mathrm{kg}$ ) and tested for their effects. Treatments were given using a within-subject Latin square design (Appendix C.2).

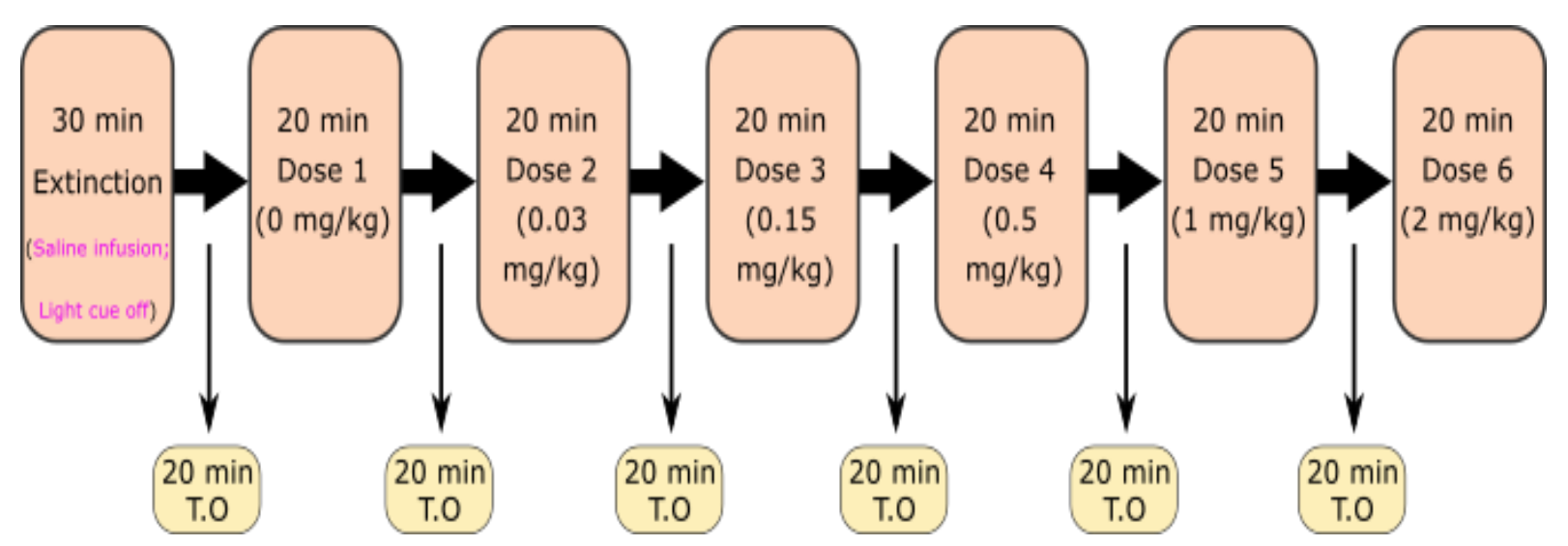

\section{Figure 2.3: Dose-response cocaine self-administration procedure schematic diagram}

The dose-response cocaine self-administration test begins with an extinction phase where the light cue is removed and heparinised saline infusion is delivered to the rats. Following a 20 min T.O period rats are allowed to self-administer a range of cocaine doses $(0,0.03,0.15,0.5,1$ and 2 $\mathrm{mg} / \mathrm{kg}$ ) during which the light cue was turned on. Between each exposure to a cocaine dose, a 20 min T.O period was included for changing of the cocaine doses. Upon stable responding and achieving the criteria rats were injected with either vehicle or MP1104 and tested for the number of active lever responses during the whole session. Cocaine or saline infusions were delivered to rats on a FR2 schedule for the whole session. 


\subsection{Rotating disk electrode voltammetry}

To measure the effects of MP1104 on DAT function (a crucial mechanism that helps in the reuptake of $D A$, see section 1.2 .2 .2 ) we used the rotating disk electrode voltammetry (RDEV). RDEV is used to measure monoamine transporter function (Earles and Schenk, 1998). The mechanism involved for the working of RDEV includes that a sufficient oxidation potential causes 'electroactive' substances with oxidisable chemical moieties to transfer electrons at the electrode's surface, generating a detection current proportional to the amount of material oxidized (Hagan et al., 2010). RDEV has been used to study uptake for several transporters, including DAT (Chen et al., 1999; Earles and Schenk, 1998; Schenk, 2002), SERT (Hagan et al., 2010) and NET (Burnette et al., 1996; Chen et al., 1998). This thesis studied the effects of MP1104 on monoamine transporter function (DAT) using RDEV in drug naïve rats.

The RDEV set-up consists of a chamber (kindly donated by J. B. Justice Jr., Professor of Chemistry, formerly Emory University) maintained at $37^{\circ} \mathrm{C}$ into which the tissue of interest was minced and placed. A rotating glassy carbon electrode (Pine Instruments, AFMDO3GC, Durham, NC, USA) is lowered into the heated chamber, and rotated at a constant speed of $2000 \mathrm{rpm}$ (see Figure 2.4). A potential relative to the $\mathrm{Ag} / \mathrm{AgCl}$ reference electrode that is sufficient to oxidise $\mathrm{DA}$ applied to the RDEV and the resulting change in current is measured. DA uptake into tissues in the chamber causes decrease in free DA in the bath, a reduction in oxidation, and a decrease in current. The eDAQ potentiostat EA161 (eDAQ, NSW, Australia) maintains a set potential (+450 mV) at the rotating glassy carbon electrode relative to the $\mathrm{Ag} / \mathrm{AgCl}$ reference electrode by adjusting the current of the auxiliary Pt electrode. The current from the potentiosat is sent into an oscilloscope and analysed using Chart recording software (Chart, e DAQ, NSW, Australia). The rotation of the RDEV at constant angular velocity causes DA in solution to approach perpendicular to the electrode, oxidise at the electrode surface and propel away parallel to the electrode. During this process two electrons are released that can be measured and the total concentration of DA in the chamber can be determined according to the Levich equation, as below:

$$
i_{L}=0.62 n F A D^{2 / 3} v^{-1 / 6} \omega^{1 / 2} C
$$

where $i_{L}$ is the limiting current in $\mathrm{mA}, \mathrm{n}$ is the number of electrons transferred per mole of analyte, F is Faraday's constant $\left(96,485\right.$ coulombs/equivalent of electrons), $A$ is the electrode area in $\mathrm{cm}^{2}$, $D$ is the diffusion coefficient in $\mathrm{cm}^{2} / \mathrm{s}, \mathrm{v}$ is the kinematic viscosity of the solution in $\mathrm{cm}^{2} / \mathrm{s}, \omega$ is the 
angular velocity of rotation in radians/s (where $\omega=2 \pi N, N$ is in rotations/s) and $C$ is the concentration in mM (Earles and Schenk, 1998).

The zero trans and low to infinite trans RDEV models that are used to determine the kinetic values of monoamine transporters such as DAT were performed using previously established protocols (Povlock and Schenk, 1997; Simonson et al., 2015). Prior to each experiment, the carbon electrode was carefully polished clean for 1 min using a Buehler microcloth with a slurry of alumina polish ( $0.05 \mu \mathrm{m}$ diameter aluminium slurry; Buehler, IL, USA) and milliQ water, followed by milliQ water only to remove remaining slurry. This polishing sequence was carried out at the beginning and end of each experimental day and polishing using miliQ water between each sample. At the beginning of each RDEV experiment, a standard concentration curve was performed by adding increasing concentrations $(0.25,0.5,0.75,1,1.5 \& 2 \mu \mathrm{M})$ of DA in KREBS buffer (see Appendix A) to produce total concentrations of $0.25,0.75,1.5,2.5,4 \& 6 \mu \mathrm{M}$ in volume range of $300-320 \mu \mathrm{L}$ in the RDEV chamber. Linear regression was performed on increase in current $(n A)$ values following each DA addition. The $\mathrm{R}^{2}$ value was required to be $\geq 0.95$ to be accepted for analysis. 


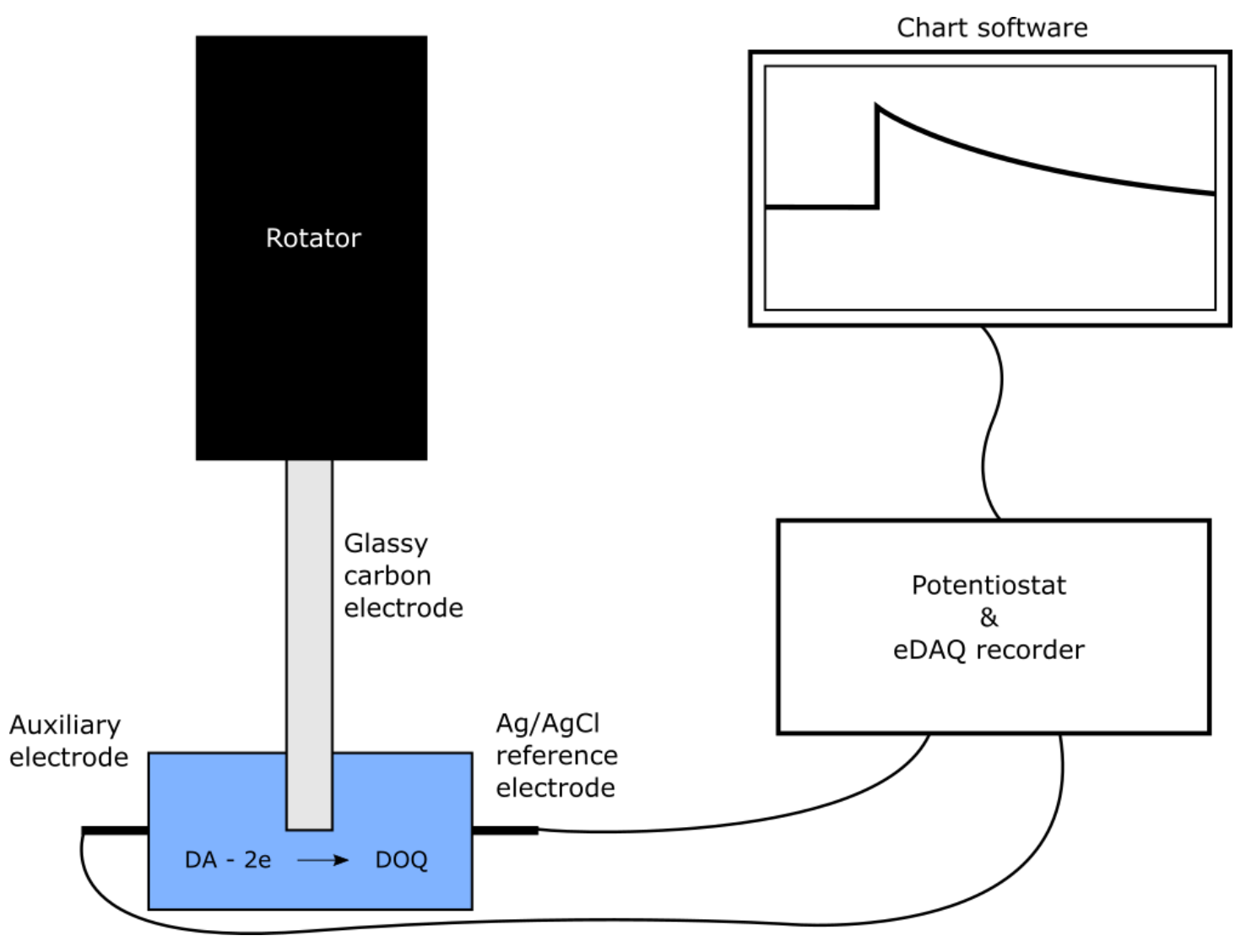

\section{Figure 2.4: RDEV experimental set-up}

Minced tissue from the rat dStr or NAc was placed into the RDEV chamber and the glassy carbon electrode rotated at $2000 \mathrm{rpm}$. $\mathrm{A}+450 \mathrm{mV}$ potential was applied relative to the $\mathrm{Ag} / \mathrm{AgCl}$ electrode to oxidise dopamine (DA) to DA quinone (DOQ). Electrons released from this reaction flow to the surface of the electrode where they are measured as a current (by the eDAQ recorder). The rate of DA uptake by DAT was measured from the slope of the line on chart software. Uptake of DA into tissue via DAT decreases the number of available electrons (measured as current), providing a linear estimate of DA concentration in the tissue chamber. 


\subsubsection{Dissection of brain tissue}

Drug naïve rats were euthanized via $\mathrm{CO}_{2}$ asphyxiation and decapitated. The brain was rapidly removed (within 1-2 $\mathrm{min}$ ) and the specific regions of interest (dStr and NAc) were dissected using a brain matrix (Alto, AgnTho's AB, Sweden) on glass petri-dish on ice. The dStr and NAc were located using coordinates in Paxinos and Watson (2005), shown in Table 2.1. The isolated tissue was weighed as follows.

Table 2.1: Dissected brain regions, their coordinates and weights

\begin{tabular}{|l|l|l|}
\hline Brain regions & Interaural coordinates & Tissue weight (mg) Mean \pm SEM \\
\hline Dorsal striatum (dStr) & +9 to +11 & $12.3 \pm 0.5$ \\
\hline Nucleus accumbens (NAc) & +9 to +11 & $9.7 \pm 0.2$ \\
\hline
\end{tabular}

Values presented as mean \pm SEM.

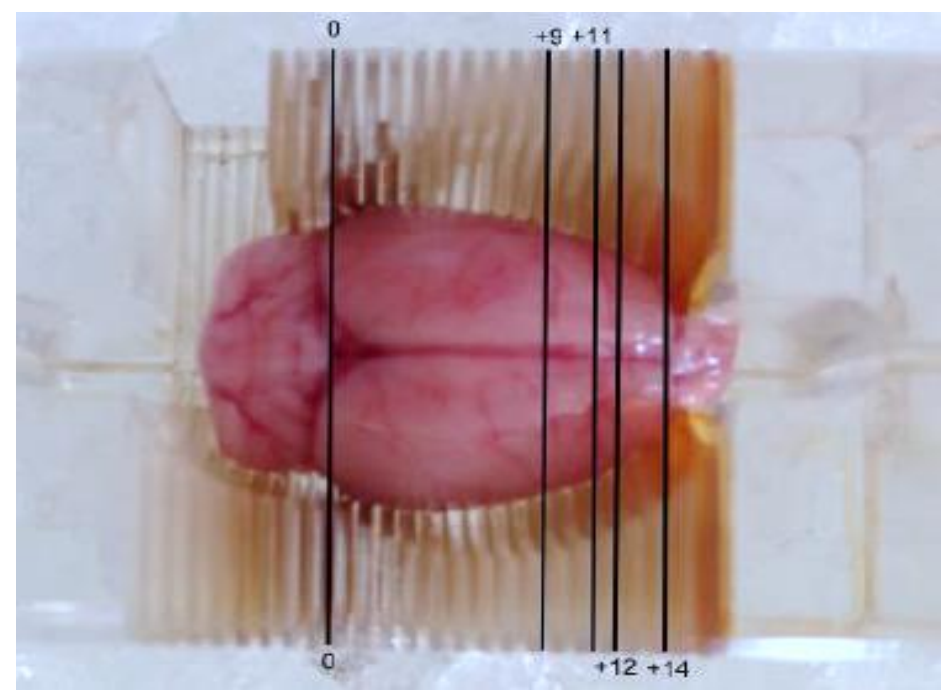

Figure 2.5: Interaural coordinates for rat brain dissection

Rat brains were removed and positioned in the brain matrix to measure interaural units. The brain regions of interest were dissected from $2 \mathrm{~mm}$ sections; dorsal striatum and nucleus accumbens from +9 to +11 and the prefrontal cortex from +12 to +14 . 
Ice-cold KREBS buffer ( $300 \mu \mathrm{L}$ ) was added to the isolated tissue (dStr or NAc) and minced quickly with razor blades and then transferred into a microcentrifuge tube. After allowing the brain tissue to settle via gravity, the KREBS buffer was removed and replaced with $300 \mu \mathrm{L}$ of prewarmed KREBS buffer $\left(37^{\circ} \mathrm{C}\right.$ ) aerated with carbogen gas (95\% O2 \& $5 \% \mathrm{CO} 2$; BOC Ltd., NZ) to maintain tissue viability. The tissue was washed eight times in aerated KREBS buffer allowing the tissue to settle between each wash. The minced tissue ( $d S \operatorname{tr}$ or NAc) pieces were suspended in the RDEV chamber (maintained at $37^{\circ} \mathrm{C}$ ) in a volume of $294 \mu \mathrm{L}$. Carbogen gas was allowed to flow over the surface of the chamber to provide sufficient oxygen for the tissue to remain viable throughout the duration of the experiment. The glassy carbon electrode was lowered into the chamber and rotated at $2000 \mathrm{rpm}$. A potential of $+450 \mathrm{mV}$ relative to $\mathrm{Ag} / \mathrm{AgCl}$ reference electrode was applied to the electrode by the potentiostat and allowed to run until a stable baseline recording was reached. DA was then added to the tissue suspension. In the zero trans model, single additions DA was added and the current measured for $30 \mathrm{~s}$, whereas in the low to infinite trans model ascending concentrations of DA were added, with the current allowed to reach baseline between each addition. The resulting current data was recorded using eDAQ Chart software.

Approximately $10 \mathrm{~s}$ of DA uptake data was recorded and analysed, via linear regression of the change in current. Linear regression analyses with an $\mathrm{R}^{2}$ value of less than 0.95 was not used in the study. The slope of regression was normalised to the standard concentration curve obtained prior to tissue recordings and the weight of rat brain tissue was used to obtain uptake values in $\mathrm{pmol} / \mathrm{s} / \mathrm{g}$. DA uptake values were fitted to the Michaelis-Menten curve (One-site binding analysis) for the kinetic studies and the values for $V_{\max }$ and $K_{m}$ were obtained for each tissue taken from an individual rat. 


\subsection{Anti-pain assays}

\subsubsection{Warm-water tail withdrawal assay}

This assay was conducted as previously described (Paton et al., 2017; Simonson et al., 2015; Thorn et al., 2011). Mice (used to measure cumulative dose-response tail withdrawal effects) were restrained in plexiglass restrainers with an internal diameter of $24 \mathrm{~mm}$, whereas rats (used to measure both cumulative dose-response and time-course tail withdrawal effects) were restrained by hand. Tail withdrawal latencies were measured by immersing a third of the distal portion of the tail in a water-bath containing $50 \pm 0.5^{\circ} \mathrm{C}$ (mice) or $55^{\circ} \mathrm{C}$ (rats) heated water. The time taken for withdrawal responses were recorded using a hand-operated stopwatch. To avoid tissue damage, a maximum of $10 \mathrm{~s}$ (mice) or $15 \mathrm{~s}$ (rats) was used as a cut-off latency.

\subsubsection{Time-course tail withdrawal effects}

One-third of the rats tail was submerged into $55^{\circ} \mathrm{C}$ water, and the latency to withdraw the tail recorded. Three baseline values were obtained with a 5 min time interval between each recording. The rats were then injected with either vehicle, MP1104 (0.3 or $0.6 \mathrm{mg} / \mathrm{kg}$, i.p.) or morphine (10 mg/kg, i.p.) and the latencies were measured at 5, 10, 15, 30, 45, 60, 90, 120, 150, $180,240,300,360,420$, and $480 \mathrm{~min}$. The maximum possible effect (MPE) of analgesia was calculated by applying the formula:

$$
\% \mathrm{MPE}=\left(\frac{\text { test latency }- \text { baseline latency }}{15-\text { baseline latency }}\right) \times 100
$$

In order to measure whether these effects were mediated via KOPr, MOPr or DOPr, rats were pretreated $24 \mathrm{~h}$ prior with selective antagonists for KOPr (nor-BNI, $10 \mathrm{mg} / \mathrm{kg}$, s.c.), and MOPr ( $\beta-F N A$, $10 \mathrm{mg} / \mathrm{kg}$, s.c.), or $15 \mathrm{~min}$ prior to agonist administration with DOPr antagonist (NTI, $15 \mathrm{mg} / \mathrm{kg}$, s.c.). 


\subsubsection{Cumulative dose-response tail withdrawal effects}

A within-subject, cumulative dose-response experimental design was used as described previously (Bohn et al., 2000) to reduce animal numbers. Animals were given s.c. injections of MP1104 at increasing concentrations and tail-withdrawal latency measured at $1 \mathrm{~h}$ (based on the onset of action of MP1104 using time-course withdrawal test). The injections (delivered at a volume of $5 \mu \mathrm{L} / \mathrm{g}$ ) for cumulative dose-response experiments were given into the left and right flank and the scruff of the neck in sequence to prevent delivery of injections to the same site. Rats are placed into their home cage under observation between each test. MP1104 was evaluated in tail-withdrawal at the following doses: $0.03,0.1,0.3,0.6,1,3,6,10,15 \mathrm{mg} / \mathrm{kg}$ in rats, and at 0.1 , $0.3,0.6,1,3,6,10 \mathrm{mg} / \mathrm{kg}$ doses in mice. The animals were culled immediately following the last measurement. The potency, expressed as $E D_{50}$ and efficacy, expressed as $E_{\max }$ were obtained using non-linear regression analysis using GraphPad Prism software version 7 (GraphPad, La Jolla, CA).

\subsubsection{Chemotherapy-induced neuropathic pain assay}

To induce chemotherapy-induced neuropathic pain (CINP), male C57BL/6J mice were administered the taxane chemotherapy drug, paclitaxel ( $4 \mathrm{mg} / \mathrm{kg}$, i.p. at $10 \mu \mathrm{L} / \mathrm{g})$, four times on alternate days to give a cumulative dose of $16 \mathrm{mg} / \mathrm{kg}$ (see Figure 2.6). This dosing regime is known to produce paclitaxel-induced neuropathic pain in mice (Deng et al., 2015; Paton et al., 2017). Control mice were given vehicle consisting of a 1:1:18 ratio of absolute ethanol, cremophor EL, and physiological saline respectively and housed in separate cages to paclitaxel treated mice. To measure the progress of paclitaxel induced effects, mice were placed on an elevated mesh grid chambers with holes of approximately $1 \mathrm{~mm}$ and assessed for responses to both mechanical and cold allodynia. Following habituation to the apparatus, each hind paw was measured twice for each stimulation, starting with mechanical. Both stimulations of allodynia were evaluated on alternate days to assess the effects induced by paclitaxel in mice. On days when paclitaxel dose was to be administered measurements were taken prior to the administration of paclitaxel. 


\subsubsection{Mechanical allodynia}

Mechanical allodynia was measured using von Frey filaments (20 piece set; \#58011, Stoelting, IL, USA). Von Frey filaments numbered from 2 to 9 were used to measure the sensitivity to a mechanical stimulus. The filaments, beginning with filament 5 , were pressed at a right angle against the plantar region of the right and left hind paw of mice with force enough to produce a bend in the filament. The filament was placed on the hind paw of mice for approximately $3 \mathrm{~s}$ or until a positive response (defined as a sharp withdrawal of the paw) was recorded. A simplified up-down method was used according to published methods (Bonin et al., 2014). Briefly, a positive response to filament application resulted in the use of next lower filament in the subsequent test, but if no response was observed, the next higher filament was used. The process continued until five filaments were used and final threshold calculated. The paw withdrawal threshold was calculated by using the outcome of the fifth filament used in each testing sequence, and adding or subtracting a value of 0.5 filament intervals if the response to the fifth filament was negative or positive, respectively (Bonin et al., 2014) (see Appendix E for examples of simplified up-down von Frey filament testing scheme).

\subsubsection{Cold allodynia}

Allodynia is described as pain due to a stimulus that does not normally provoke pain (Loeser and Treede, 2008). On days $0,2,4,6,7,9,11,13,15$ cold allodynia was measured according to previously described methods (Deng et al., 2015) (see Figure 2.7). A drop of acetone (created using a $1 \mathrm{~mL}$ syringe) was administered to the plantar surface of the hind paw ensuring no mechanical force is caused with the end of the syringe. Total response time of behaviours that included licking, elevating, biting or shaking of paw, were measured. The experiment was repeated on the other hind paw following a 5 min intervals and a total of two measurements taken for each hind paw. Cold allodynia for each animal was recorded by calculating the average response time (s) from the four measurements. 


\subsubsection{Evaluation of acute MP1104 administration on chemotherapy-induced neuropathic}

pain

To evaluate the effects of MP1104 on CINP, mice were administered cumulative doses of MP1104 $(0.1,0.2,0.3,0.5,1,3,6 \mathrm{mg} / \mathrm{kg} / \mathrm{s} . \mathrm{c}$.$) , on day 15$ following stable induction of CINP using withinsubject design (Figure 2.6). Effects were evaluated alongside morphine (0.5, 1, 2, 5, 10, 15, 20 $\mathrm{mg} / \mathrm{kg} / \mathrm{s.c.}$ ), a clinically available analgesic. MP1104, morphine or equivalent volumes of vehicle (DMSO:Tween-80:saline in a ratio of 2:1:7) was administered to the mice with a $1 \mathrm{~h}$ interval between each cumulative dose. Non-linear regression analysis was used to calculate $\mathrm{ED}_{80}$ and $E_{\max }$

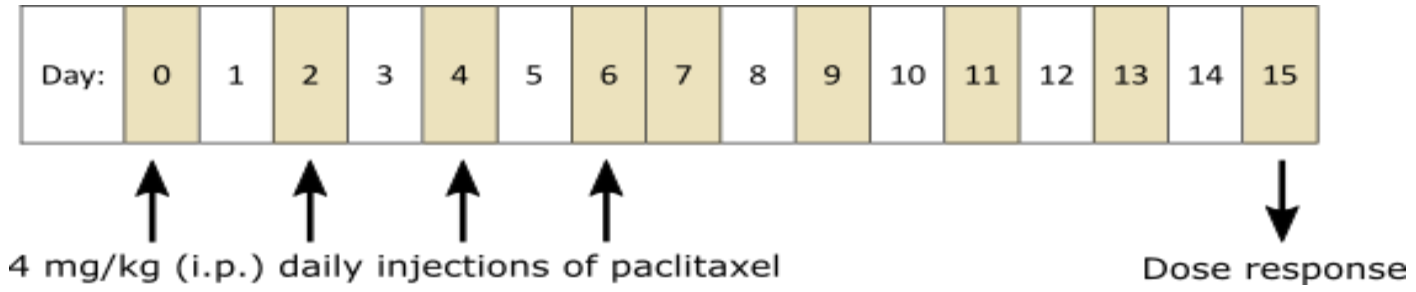

Figure 2.6: Schematic diagram of experimental design for acute dose-response procedure in paclitaxel treated mice

Mice were administered $4 \mathrm{mg} / \mathrm{kg}$ i.p. of paclitaxel on days $0,2,4$ and 6 . The shaded days indicate when animals were assessed for mechanical and cold allodynia. On days where allodynia measurements and paclitaxel administration both occurred, measurements were always taken prior to injections. The dose-response experiments were carried out on day 15 , following the baseline mechanical and cold allodynia measurements. 


\subsubsection{Evaluation of chronic MP1104 administration on chemotherapy-induced neuropathic pain}

The effects of repeated MP1104 administration on mechanical and cold allodynia were evaluated in mice with established paclitaxel-induced neuropathic pain. This experimental design allowed the effects of tolerance following chronic administration of MP1104 in comparison to morphine.

On day 15, CINP mice were carefully allocated into treatment groups to ensure equivalent average mechanical allodynia score across all groups. All groups were administered daily injections of either MP1104 (1.2 mg/kg, i.p.) or morphine (10 mg/kg, i.p.) from days 16 - 38 (since morphine started to produce stable tolerance by this day). The experimenter was blinded to the treatments each animal received. On all odd numbered days, both mechanical and thermal allodynia measurements were performed prior to drug administration treatments, whereas on even days drug was administered $1 \mathrm{~h}$ prior (based on the onset of action of MP1104 using time-course withdrawal test) to mechanical and cold allodynia testing (Figure 2.7). 


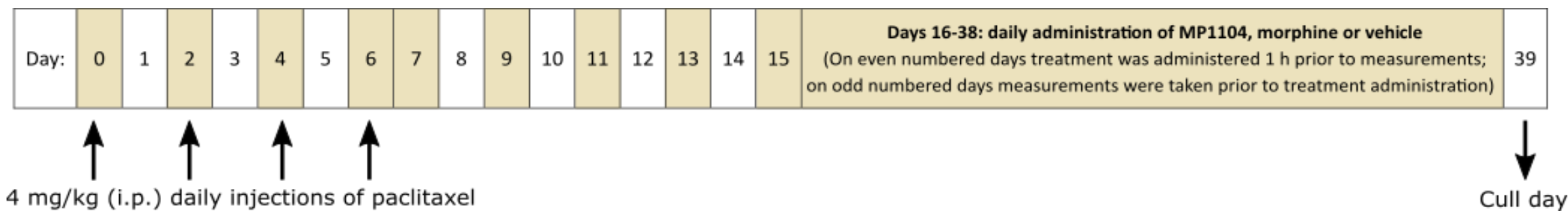

Figure 2.7: Schematic diagram of experimental design for chronic treatment of MP1104 or morphine in mice with established paclitaxel-induced neuropathic pain

Experimental design for the evaluation of chronic drug treatment in mice following paclitaxel-induced neuropathic pain induction. Drug was administered on days 16-38. On Day 39 the sciatic nerve, lumbar spinal cord and dorsal root ganglion tissues were collected. The shaded days indicate when animals were assessed for mechanical and cold allodynia. On days where allodynia measurements and paclitaxel administration both occurred, measurements were always taken prior to paclitaxel administration. 


\subsection{Side effects behavioural assays}

\subsubsection{Spontaneous locomotor activity test}

The sedative effects of MP1104 were evaluated by measuring spontaneous locomotor activity in male Sprague Dawley rats using previously described methods (Morani et al., 2012). The apparatus consists of open activity chambers equipped with infrared beams (Med Associates: ENV-520; SOF-811). Rats are placed in the middle of the chamber and their movement recorded via infrared beam breaks. Rats were habituated in the activity chambers for $\mathbf{3 0} \mathrm{min}$, then injected with either vehicle (DMSO:Tween-80:Milli $Q$ water in a ratio of 2:1:7) or MP1104 (0.3, 0.6 or 1 $\mathrm{mg} / \mathrm{kg}$, i.p.) and put back in the activity chamber for $60 \mathrm{~min}$. Stereotypic and ambulatory activity was recorded during both habituation and testing sessions. A single ambulatory count was calibrated to be recorded when three consecutive horizontal infrared beam breaks were made by the rat. Stereotypic behaviour was defined by the software as any horizontal movement detected below this threshold. All tests were performed in the dark between 9:00 and 17:00 $\mathrm{h}$.

\subsubsection{Conditioned place aversion}

Conditioned place aversion (CPA) was performed following previously described methods (Tejeda et al., 2013) in male Sprague Dawley rats. The three-chamber place preference apparatus (PanLab, Harvard Apparatus, USA) consisted of two large chambers (30 x $30 \times 34 \mathrm{~cm}$ ) connected by a smaller corridor $(8 \times 10 \times 34 \mathrm{~cm})$ separated by sliding doors. One large chamber had a smooth white floor with black walls and a white stripe pattern while the other had a texture black floor with white walls with a black dot pattern and an average light intensity of 20 lux in each conditioning chamber (see Figure 2.8). The corridor was a neutral zone with grey walls and floor illuminated at an intensity of 70 lux to avert lingering of the animal. The CPA procedure took place over 9 days. Day 0 was the habituation day where rats were habituated to the CPA apparatus for 15 min. Day 1 was the pre-conditioning day, where rats were allowed free access to both chambers for $15 \mathrm{~min}$. All activities were tracked using SMART 3.0 software (PanLab). Animals that showed over $80 \%$ preference for a particular chamber or over $40 \%$ preference for the corridor were excluded from testing. On days 2-7 conditioning was conducted for $45 \mathrm{~min}$ using a biased procedure, whereby injections (i.p.) of MP1104 agonist $(0.3$ or $0.6 \mathrm{mg} / \mathrm{kg}$ ) or U50,488 $(10 \mathrm{mg} / \mathrm{kg}$ ) or vehicle was followed by confining the animal to the preferred chamber with vehicle injections in the least preferred chamber, in a counterbalanced manner. The DOPr antagonist, NTI (15 
$\mathrm{mg} / \mathrm{kg}$, s.c.) was injected $15 \mathrm{~min}$ prior MP1104. The post-conditioning test was performed on day 8. Rats were placed in the corridor and allowed free access to both chambers for $15 \mathrm{~min}$ and time spend in each chamber recorded using SMART 3.0 software (PanLab). Time spent in each chamber on pre-conditioning and post-conditioning days were compared to determine changes in preference. The total distance travelled by rats following treatment administration was also measured.

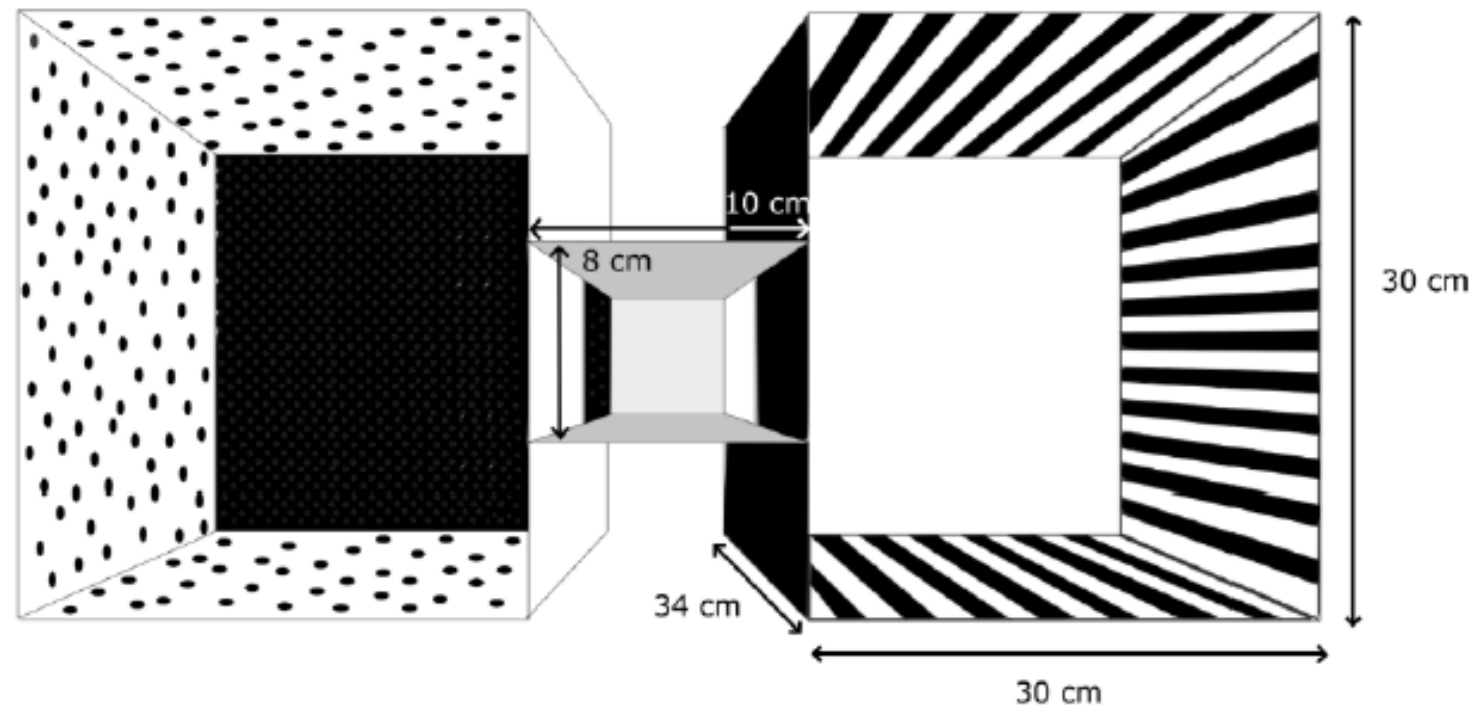

Figure 2.8: Conditioned place aversion apparatus

The three-chambered conditioned place aversion apparatus is shown above. The black coloured floor is textured while the white coloured floor is smooth. On Day 0 rats were habituated to the apparatus. Pre-conditioning test day (Day 1) was the next day, where rats were given free access to both the chambers in order to obtain a baseline preference. Rats were divided into treatment groups (vehicle or MP1104 or U50,488 or NTI/MP1104). Days 2-7 were conditioning phase days where animals were confined to one compartment and received either systemic vehicle or U50,488 or MP1104 or NTI/MP1104 treatment in their most preferred box (Days 2, 4, and 6) or vehicle in their least preferred box (Days 3, 5, and 7). The effect of the treatment was then assessed in the post-test by allowing access to the entire apparatus as in the pre-test (Day 8). 


\subsubsection{The elevated plus maze test of anxiety}

Time spent in the open arm of an elevated plus maze (EPM) is used as a preclinical model of anxiety (Walf and Frye, 2007). The EPM was made of black plastic and consisted of four arms (50 $\mathrm{cm} \times 10 \mathrm{~cm}$ each) elevated $55 \mathrm{~cm}$ above the ground (see Figure 2.9). The two open arms consisted a small clear perspex parapet measuring $2.5 \mathrm{~cm}$ in height and the two closed arms were $40 \mathrm{~cm}$ high black walls. Rats were administered MP1104 (0.3 or $0.6 \mathrm{mg} / \mathrm{kg})$, yohimbine $(2.5 \mathrm{mg} / \mathrm{kg}$ ) or vehicle (DMSO, tween 80 and milliQ water in a ration 2:1:7) injections (i.p.) and placed in the center of the apparatus facing an open arm. The total time spent by rats on each open arm and the total arm (closed and open) entries were recorded for $5 \mathrm{~min}$ by an investigator blinded to the treatment groups (Sony HDR-SR5E digital camera recorder). Open arm time was calculated when rats had all four paws on the open arm.

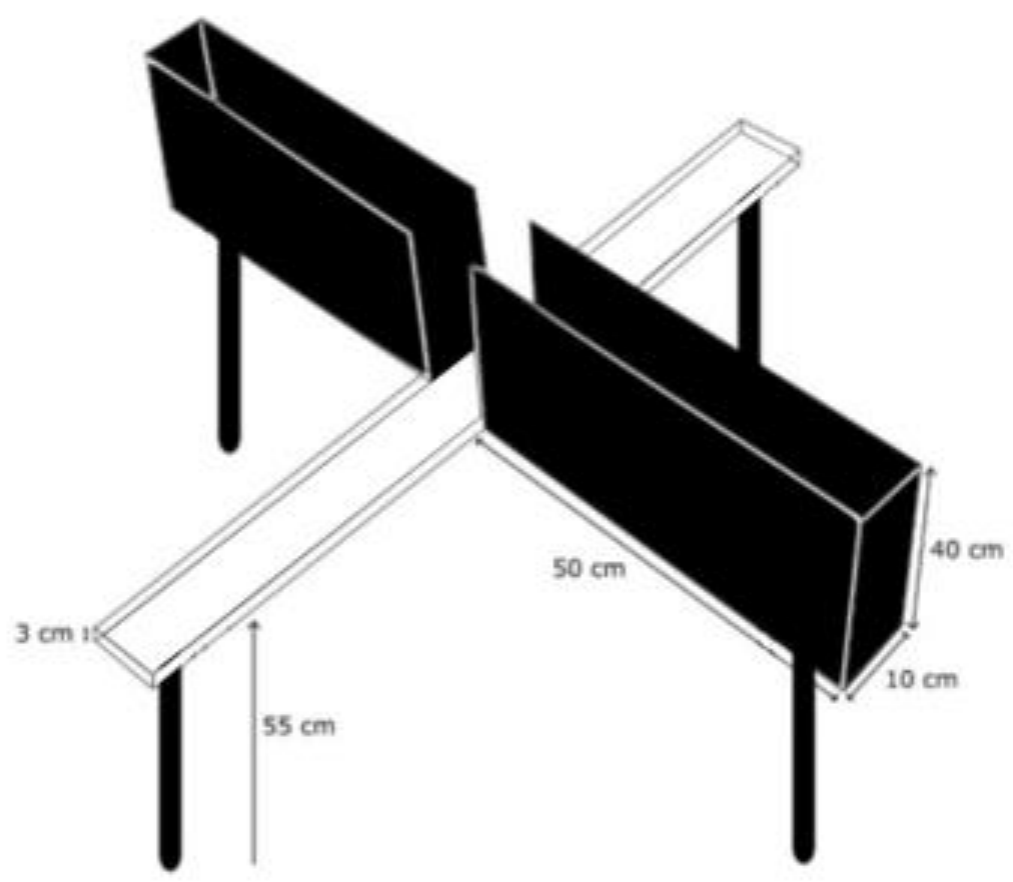

\section{Figure 2.9: Elevated plus maze apparatus}

Anxiogenic effects were examined in the EPM test. Animals were injected with either vehicle or MP1104 or yohimbine prior to placing them on the centre of the maze. Time spent on the open arm were used as anxiolytic behavioural measures, while closed arm entries were used to test for anxiogenic effects. 


\subsubsection{Forced swim test}

The forced swim test (FST), a preclinical measure of pro-depressive effects was performed using a cylindrical swim chamber $44 \mathrm{~cm}$ in height and $20 \mathrm{~cm}$ in diameter, as previously described (Slattery and Cryan, 2012). Water was maintained at a temperature of $25 \pm 1^{\circ} \mathrm{C}$ and filled to a depth of $35 \mathrm{~cm}$. Before the testing day, rats were habituated to forced swimming behaviour for $15 \mathrm{~min}$. On the test day rats were injected with MP1104 ( 0.3 or $0.6 \mathrm{mg} / \mathrm{kg} / \mathrm{i}$. . .) or vehicle (DMSO, tween 80 and milliQ water in a ration 2:1:7) before a 5-min swim session. All test sessions were recorded using a Sony HDR-SR5E digital camera recorder. Test videos were scored as displaying either climbing, swimming or immobility using SMART 3.0 software (PanLab).

\subsection{Statistical analysis}

All statistical analyses were performed using GraphPad Prism software version 7 (GraphPad, La Jolla, CA). Data is expressed as the mean \pm standard error of mean (SEM). For cocaine-primed reinstatement tests, data was analysed using one-way analysis of variance (ANOVA) with repeated measures with Bonferroni's post-test. Data for multiple dose cocaine selfadministration was analysed using two-way ANOVA followed by Bonferroni's multiple comparisons test. DA uptake measured by single addition of DA using RDEV techniques were analysed using one-way ANOVA and post-test analysis determined by Fisher's least significant difference (LSD) test. For the low to infinite trans model, uptake values were entered into GraphPad Prism and a non-linear regression (one-site binding hyperbole) was fitted to each repeat and the $\mathrm{V}_{\max }$ values obtained.

Two-way repeated measures ANOVA followed by Bonferroni post-tests were used for analysing significance compared to controls in the warm-water tail withdrawal tests. For the dose-response effects the data was transformed to logarithmic values (base 10), before a non-linear regression was performed in order to relate the increase in dose and the effect on tail withdrawal, mechanical or cold allodynia using a dose-response curve in GraphPad Prism software. The four parameter variable slope with least squares ordinary fit was used to fit the curve to the data sets. For mechanical allodynia, the top constraint was set to no more than 9.5 (maximal value possible) and for cold allodynia, the bottom constraint was set at no less than 0 . No constraints were set 
for tail withdrawal analysis. Using the null hypothesis that one curve fits all data sets, the extra sum-of-squares $F$ test with the bottom, top, $\log E D_{50}$ and hillslope parameters was used to compare the treatment curves. The $\mathrm{ED}_{50}$ and $\mathrm{E}_{\max }$ values were then calculated. The data sets were tested prior to statistical analysis for normality using the D'Agostino and Pearson omnibus normality test.

For locomotor activity tests, repeated measures two-way ANOVA was used to evaluate time course effects, and one-way ANOVA to compare total ambulatory and stereotypic counts. CPA data was analysed using Student's $t$-test, whereas a one-way ANOVA was used to analyse the EPM test. In FST experiments immobility, swimming and climbing behaviours in rats were analysed by two-way ANOVA, and mobility or immobility behaviours were analysed using One-way ANOVA. Bonferroni's post-test was used where applicable. Values of $p<0.05$ were considered to be statistically significant. 


\section{Chapter 3: Results}

\subsection{Anti-Cocaine effects}

\subsubsection{Effect of MP1104 on cocaine-induced drug seeking}

Following training on a FR1 schedule of reinforcement, drug naïve male Sprague-Dawley rats selfadministered cocaine and acquired self-administration behaviour. The criteria for achieving acquisition was at least 20 active lever presses within a $2 \mathrm{~h}$ cocaine self-administration period (with an active:inactive lever ratio of $\geq 2: 1$ ). This acquisition criteria was achieved by all rats by day 7 (Figure 3.1A). Rats then progressed to an FR2 schedule of reinforcement, whereby rats were required to press at least 40 active lever responses for 3 consecutive days (with an active:inactive lever ratio of $\geq 2: 1$ ) followed by a FR5 schedule of reinforcement (See 2.4.4: Self-administration training).

Once the active:inactive lever responses remained stable (Figure 3.1B), rats were subjected to a extinction phase, followed by cocaine-primed reinstatement. The effects of MP1104 were evaluated by measuring changes in reinstatement behaviour, compared to vehicle. Figure $3.1 \mathrm{C}$ shows the number of active lever responses during the three phases of the experiment, baseline, extinction and reinstatement. During the extinction phase, when cocaine was removed, the lever presses were significantly extinguished to less than $10 \%$ of baseline responses (producing $12 \pm 2$ active lever responses (mean \pm SEM) compared to baseline $179 \pm 22$ active lever responses; $p<$ 0.0001 , repeated measures ANOVA with Bonferroni's post-test (Figure 3.1C)). Following exposure to a priming injection of cocaine, rats significantly increased their responding on the active lever (162 \pm 15 active lever responses; $p<0.0001$, repeated measures ANOVA with Bonferroni's posttest; Figure 3.1C). 
A

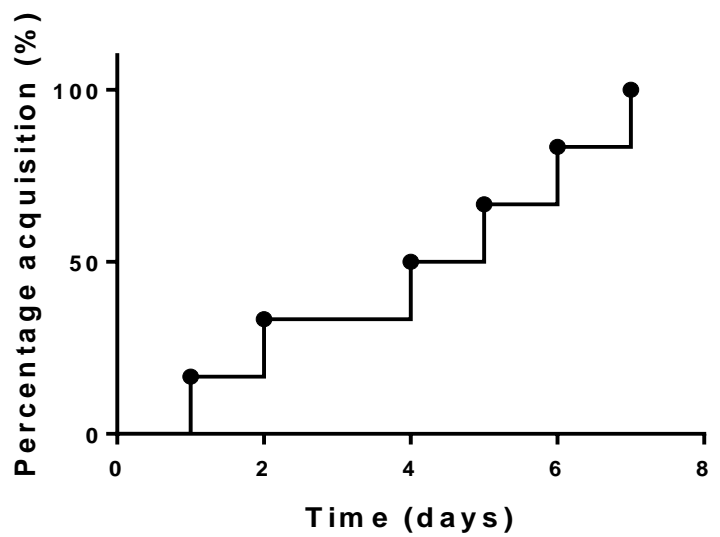

B

- Active lever responses

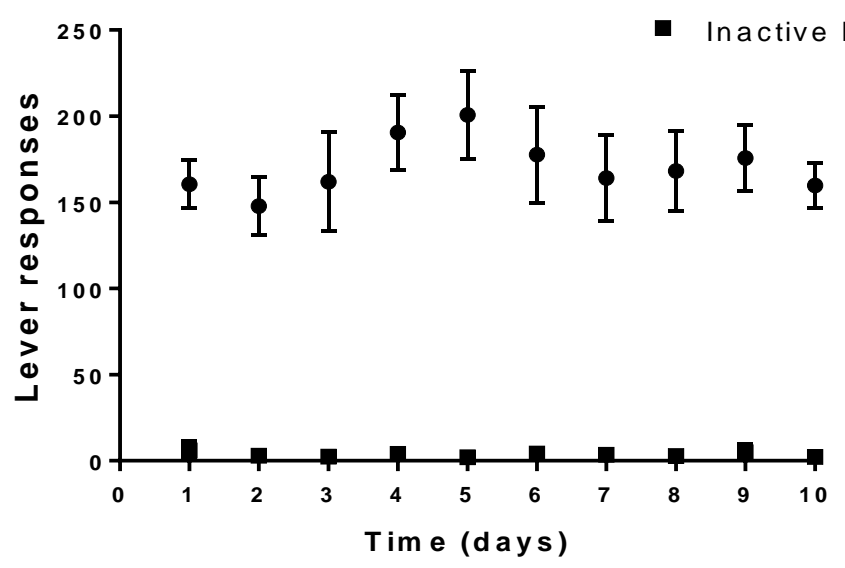

C

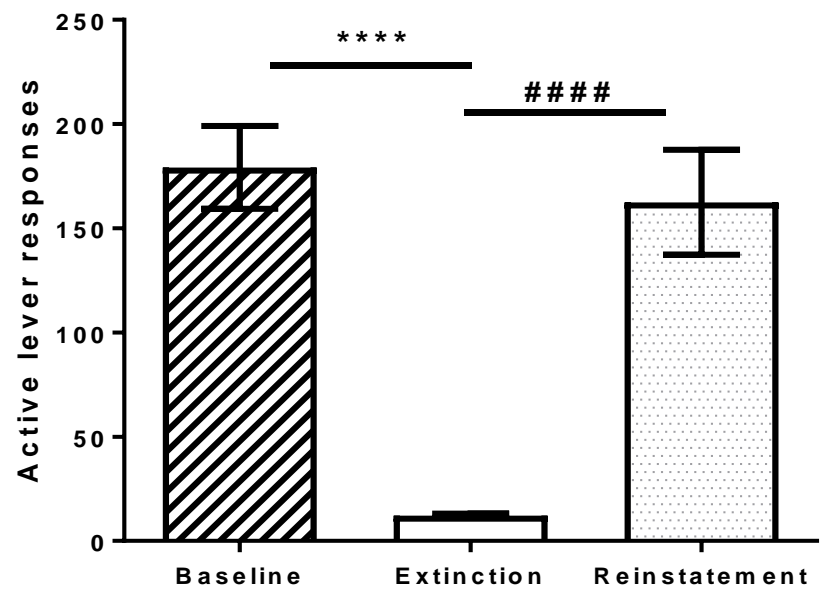




\section{Figure 3.1: Cocaine self-administration training}

(A) All rats achieved acquisition criteria by day 7, with the earliest rat achieving the behaviour on day 1. (B) Throughout the self-administration training (FR 5), the number of active and inactive lever responses remained stable with an active:inactive lever ratio of $\geq 2: 1$. (C) During the extinction phase the number of active lever responses reduced significantly compared to baseline $(p<0.0001)$, and a priming injection of cocaine $(20 \mathrm{mg} / \mathrm{kg}$, i.p.) increased the active lever presses significantly in the reinstatement phase. $(p<0.0001$, repeated measures ANOVA with Bonferroni's post-test). ${ }^{* * * *} p<0.0001$ compared to baseline, \#\#\#\# $p<0.0001$ compared to extinction. Values expressed as mean \pm SEM, $n=9$. 
In order to avoid treatment bias, once a stable baseline is established on FR5 $(<20 \%$ variation in active lever responses), rats $(n=9)$ received treatments according to a within-subject, Latin square design, whereby rats are administered treatments in a counter-balance manner (See Appendix C.1). However, selective KOPr (nor-BNI) and DOPr (NTI) antagonists were administered at the end in the counter-balanced design due to the long-lasting antagonist effects of nor-BNI (Endoh et al., 1992).

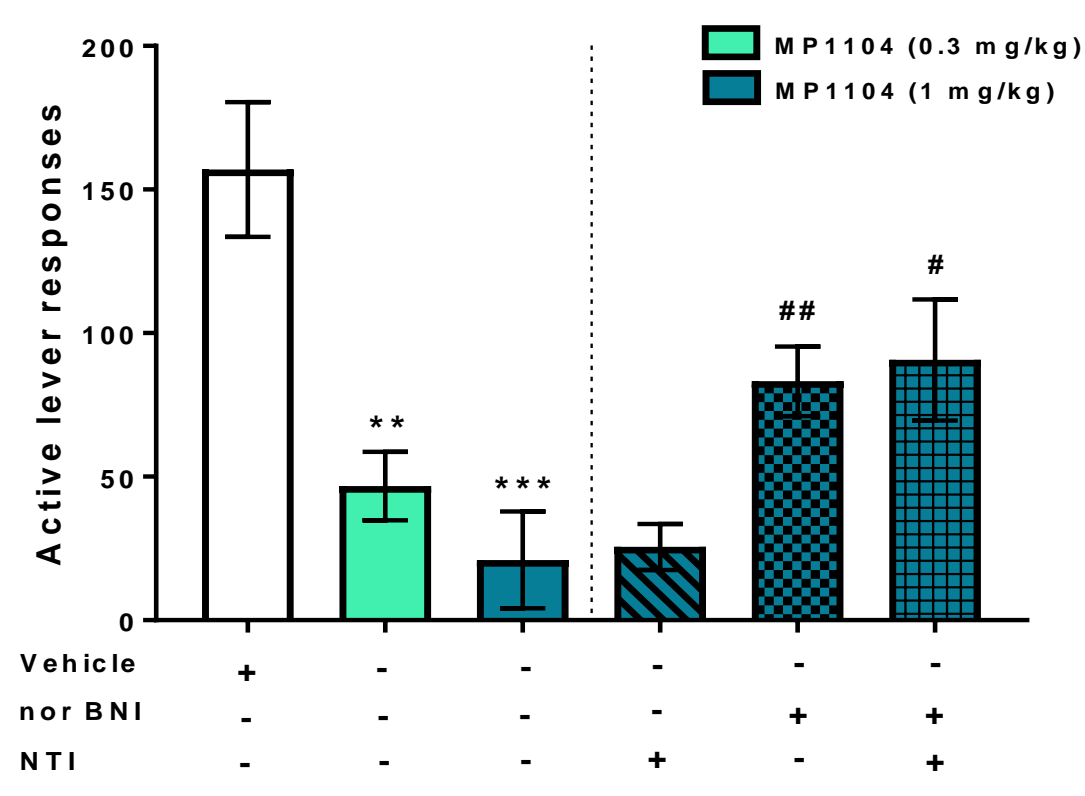

\section{Figure 3.2: MP1104 attenuates cocaine-primed reinstatement of cocaine seeking in rats}

MP1104 at $0.3 \& 1 \mathrm{mg} / \mathrm{kg}$, i.p. significantly attenuated drug seeking behaviour in rats compared to vehicle-treated controls. At $1 \mathrm{mg} / \mathrm{kg}$, i.p. the drug seeking behaviour was significantly antagonised by nor-BNI (10 mg/kg, s.c.) and the simultaneous dosing of NTI (15 mg/kg, s.c.) and nor-BNI. Repeated measures ANOVA with Bonferroni's post-test. ${ }^{* *} p<0.01,{ }^{* * *} p<0.001$ compared to the vehicle; $\# p<0.05$, \#\#p < 0.01 compared to MP1104, $1 \mathrm{mg} / \mathrm{kg}$ dose. All values expressed as mean \pm SEM, $n=9$. 
MP1104 dose-dependently decreased cocaine-induced reinstatement of drug seeking behaviour. Rats treated with MP1104 $(0.3 \mathrm{mg} / \mathrm{kg})$ significantly reduced the active lever responding by $70 \%$ (see Figure 3.2). Vehicle responses were $157 \pm 58$ compared to MP1104 (0.3 mg/kg) which has $47 \pm 23$ active lever responses and MP1104 (1 mg/kg) significantly decreased active lever presses by $87 \%\left(21 \pm 19\right.$ active lever responses, $\left.\left(F_{(8,40)}=4.31\right) ; p<0.001\right)$ (Data expressed as mean $\pm S E M$; repeated measures ANOVA with Bonferroni's post-test). To assess the contribution of KOPr and DOPr signalling pathways in attenuation of drug seeking behaviours, rats were treated with selective $\operatorname{DOPr}$ (NTI;15 mg/kg, s.C.; 15 min pre-treatment time), or KOPr antagonists (nor-BNI; 10 $\mathrm{mg} / \mathrm{kg}$, s.c.; 24 h pre-treatment time). Rats pre-treated with NTI and MP1104 (1 mg/kg) showed no change in responding behaviour ( $25 \pm 16$ active lever presses) compared to treatment with MP1104 (1 mg/kg) alone (21 \pm 19 active lever responses). However, nor-BNI and MP1104 (1 $\mathrm{mg} / \mathrm{kg})\left(83 \pm 15\right.$ active lever responses; $\left.\left(F_{(5,40)}=14.92\right) ; p<0.01\right)$ and nor-BNI, NTI \& MP1104 $(1 \mathrm{mg} / \mathrm{kg})$ combined $\left(91 \pm 15\right.$ active lever responses; $\left.\left(F_{(5,40)}=10.54\right) ; p<0.05\right)$ blocked the attenuation of drug seeking behaviour exerted by MP1104 (1 mg/kg) alone (21 \pm 19 active lever responses) significantly (see Figure 3.2). This suggests that the MP1104 attenuation of cocaineprimed drug seeking behaviour is KOPr mediated, whereas DOPr has no effects on cocaine-primed reinstatement model in rats.

\subsubsection{Effect of MP1104 on cocaine dose responding in rats trained to self-administer cocaine}

Following training on a FR2 schedule of reinforcement, and stable responding to cocaine doses $(0.03,0.15,0.5,1$ and $2 \mathrm{mg} / \mathrm{kg} /$ infusion), the effects of MP1104 were evaluated using a withinsubject Latin square design, whereby rats are administered treatments in a counter-balance manner (Appendix C.2). Refer to section 2.4.4.3 for method. The results indicate that rats exhibited an inverted $U$-shaped cocaine dose-response with higher cocaine doses ( 1 and $2 \mathrm{mg} / \mathrm{kg}$ ) and lower cocaine doses ( 0 and $0.03 \mathrm{mg} / \mathrm{kg}$ ) resulting in fewer lever presses, a measure of the rewarding effects of cocaine (see Figure 3.3). 


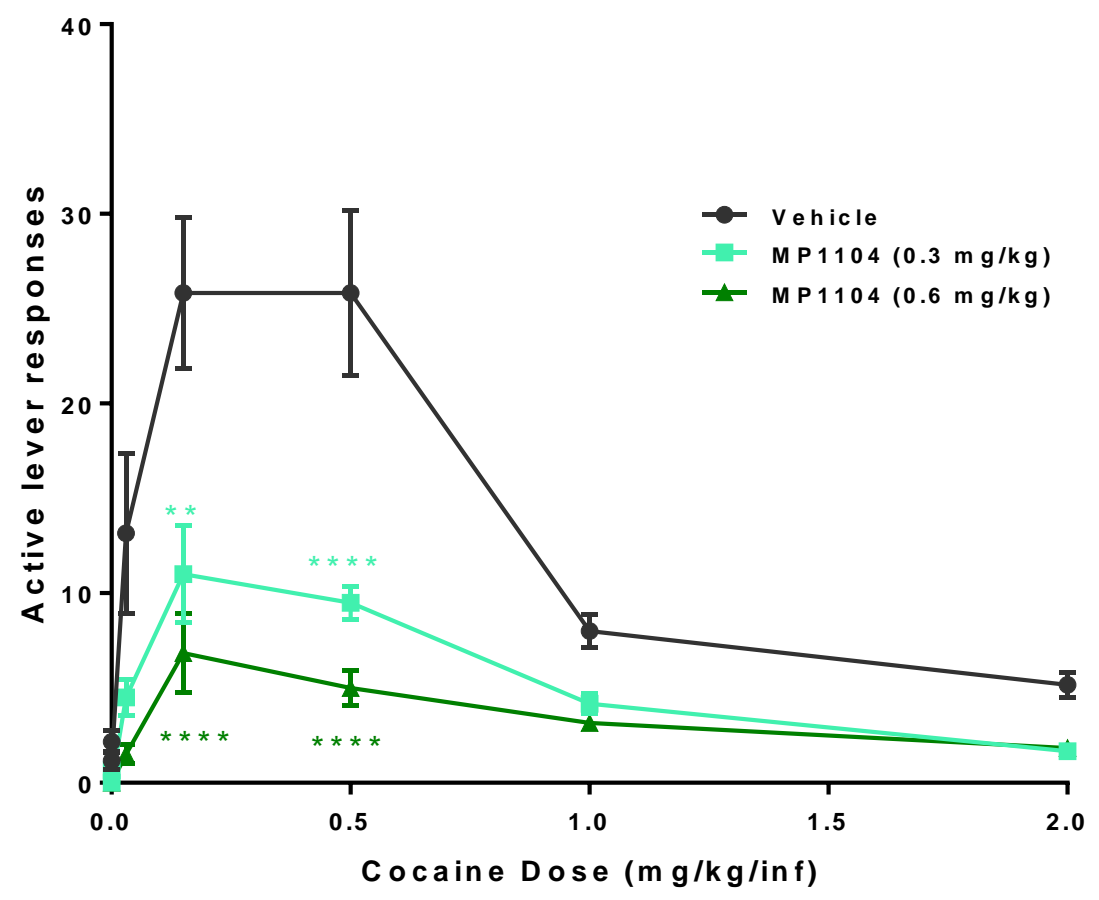

Figure 3.3: MP1104 shifts cocaine dose-response self-administration curve downwards

The effects of MP1104 on drug taking behaviour in rats was evaluated using a dose-response cocaine self-administration model. At 0.3 and $0.6 \mathrm{mg} / \mathrm{kg} / \mathrm{i} . \mathrm{p}$. MP1104 significantly shifted the cocaine dose-response self-administration curve downwards. Two-way ANOVA with Bonferroni's post-test. ${ }^{* *} p<0.01,{ }^{* * *} p<0.0001$ compared to the vehicle. All values expressed as mean \pm SEM, $n=6$. 
The average rate of responding during the 30 min extinction phase and the $20 \mathrm{~min} 0 \mathrm{mg} / \mathrm{kg}$ cocaine were $2 \pm 1$ (mean \pm SEM) and $5 \pm 2$ active lever presses respectively. The highest rate of responding was obtained at the cocaine dose of $0.15 \mathrm{mg} / \mathrm{kg} /$ infusion for both MP1104 and vehicle-treated rats. However, vehicle-treated controls also showed an equal high rate of responding at cocaine doses of $0.5 \mathrm{mg} / \mathrm{kg}$. Two-way repeated measures ANOVA revealed that MP1104 treated rats attenuated cocaine taking behaviour compared to vehicle-treated controls in dose-dependent manner $\left(\mathrm{F}_{(2,105)}=45.9 ; p<0.0001\right)$ by significantly shifting the cocaine doseresponse self-administration curve downwards. MP1104 $(0.3 \mathrm{mg} / \mathrm{kg})$ reduced active lever responses to $58 \%$ of vehicle responses, resulting in $11 \pm 3$ (mean \pm SEM) compared to vehicletreated rats (26 \pm 7 ) and a reduction of $77 \%$ was seen with the higher dose of MP1104 $(0.6 \mathrm{mg} / \mathrm{kg})$, resulting in $6 \pm 2$ active lever responses (see Figure 3.3; two-way ANOVA with Bonferroni's posttest).

In addition to these studies, we also evaluated the difference in acquisition of drug selfadministration behaviour between KOPr agonist pre-treated rats and drug naïve rats. Preliminary studies show that rats pre-treated with KOPr agonist, U50,488 (10 mg/kg, i.p.) were significantly slower in acquiring self-administration behaviour than drug naïve rats (refer to Appendix B for details).

\subsubsection{Effect of MP1104 on dopamine transporter function}

Previous studies have reported that KOPr modulates DA uptake via DAT (Kivell et al., 2014a; Simonson et al., 2015). We hypothesised that the anti-cocaine effects exerted by MP1104 are due, in part, to its ability to modulate DAT function. Therefore, to evaluate the effects of MP1104 on DA uptake via DAT, rat brain dStr and NAc tissue was taken from drug naïve rats and DAT function measured using RDEV low to infinite trans and zero trans models. 
A

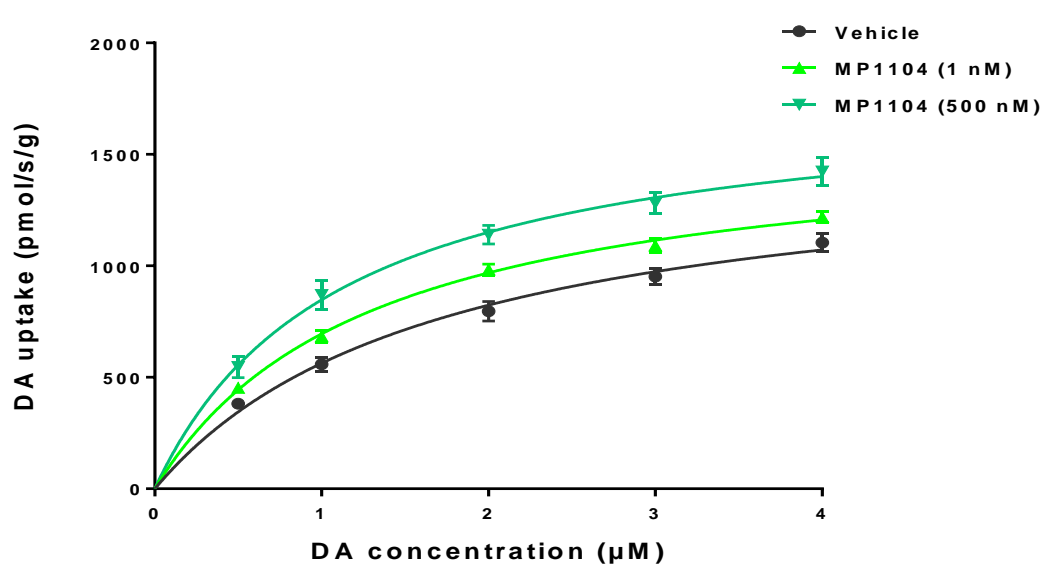

B

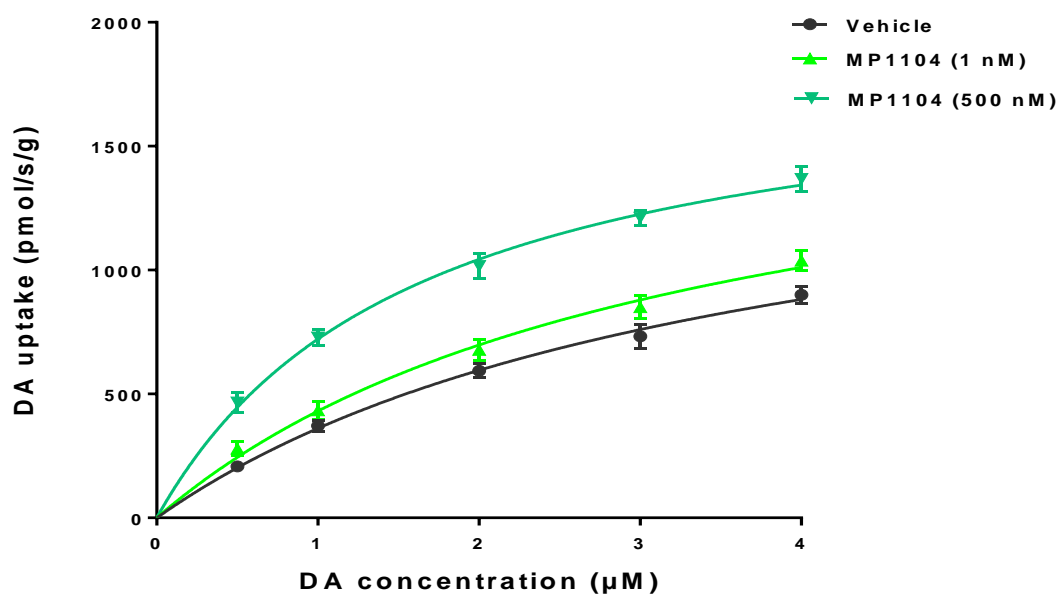


C

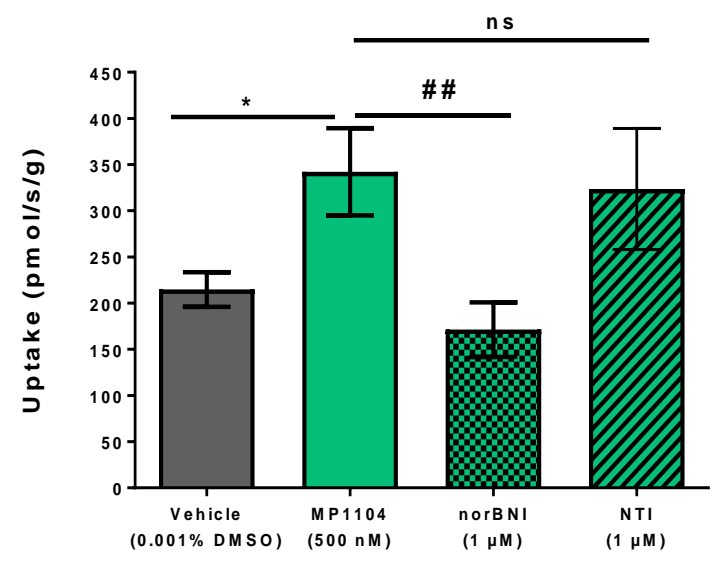

D

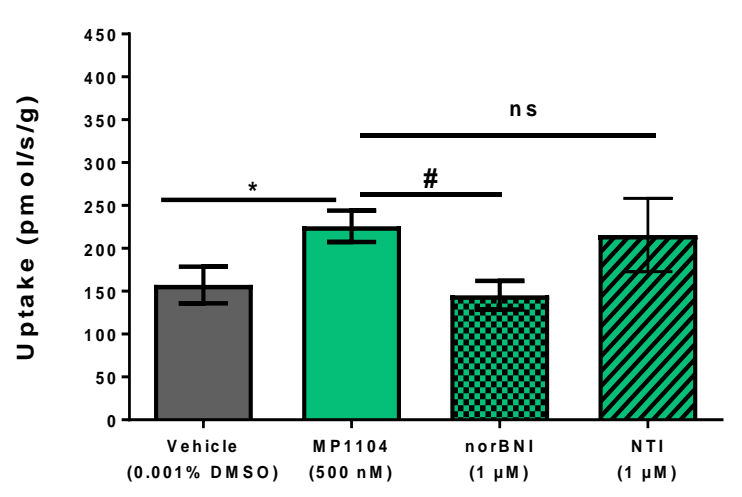

\section{Figure 3.4: MP1104 showed increase of DA uptake in rat dStr and NAc tissues}

MP1104 at $1 \mathrm{nM}$ and $500 \mathrm{nM}$ showed a significant increase in DA uptake in (A) dStr and (B) NAc compared to vehicle-treated tissue (two-way ANOVA with Bonferroni's post-test) using the low to infinite trans model of RDEV. The RDEV zero trans model was used to measure DA uptake following a single $2 \mu \mathrm{M}$ addition of DA. MP1104 (500 nM) increased DA uptake in the (C) dStr and (D) NAc. Pre-treatment of tissue suspensions with nor-BNI reversed the MP1104 induced increase in DA uptake, whereas NTI had no effect. One-way ANOVA with Fisher's LSD test, ${ }^{*} p<0.05$ compared to the vehicle; \#p<0.05, \#\#p<0.01 compared to MP1104 (500 nM). ns = nonsignificant. Values presented as mean \pm SEM, $n=5-9$ per group. 
MP1104 at $1 \mathrm{nM}(p<0.0001)$ and $500 \mathrm{nM}(p<0.0001)$ significantly increased DA uptake compared to vehicle $(0.001 \% \mathrm{DMSO})$ in $\operatorname{dStr}\left(\mathrm{F}_{(4,100)}=208.2 ; p<0.0001\right)$ and $\operatorname{NAc}\left(\mathrm{F}_{(2,100)}=155.2 ; p<0.0001\right)$ tissues (two-way ANOVA with Bonferroni's post-test) (Figure 3.4A \& B). Further analysis found that MP1104 significantly increased $V_{\max }$ (expressed as pmol/s/g) at both $1 \mathrm{nM}(1598 \pm 59.97$ $($ mean \pm SEM); $p<0.0001)$ and $500 \mathrm{nM}(1790 \pm 103.6, p<0.0001)$ compared to the vehicle control $\left(V_{\max }=1533 \pm 105.8\right)($ mean $\left.\pm S E M)\right)$ in dStr tissue. Similar effects were also observed in NAc tissue, where MP1104 at $1 \mathrm{nM}\left(\mathrm{V}_{\max }=1833 \pm 235.2\right.$ (mean $\left.\left.\pm \mathrm{SEM}\right) ; p<0.01\right)$ and $500 \mathrm{nM}\left(\mathrm{V}_{\max }=1885 \pm\right.$ 110.8 (mean \pm SEM); $p<0.0001$ ) significantly increased $V_{\max }$ values compared to vehicle control $\left(V_{\max }=1696 \pm 222.3\right.$ (mean $\left.\left.\pm S E M\right)\right)$ in minced tissue samples. The effect of the KOPr antagonist (nor-BNI; $1 \mu \mathrm{M}$ ) and DOPr antagonist (NTI; $1 \mu \mathrm{M}$ ) on the changes produced by MP1104 were evaluated using single addition of DA $(2 \mu \mathrm{M})$ using zero trans RDEV model. The increase in DA uptake by MP1104 was significantly inhibited by the KOPr antagonist in both dStr and NAc tissues to values similar to vehicle treated controls (Figure 3.4C \& D). Using single addition model, MP1104 (500 nM) produced significant increase in DA uptake (expressed as pmol/s/g) $(342 \pm 48$ (mean \pm SEM); $p<0.05)$ compared to the vehicle $(191 \pm 36$ (mean \pm SEM)) of which was significantly inhibited by nor-BNI (171 \pm 49 (mean \pm SEM); $p<0.01$ ) (Figure 3.4C). A similar trend was observed in NAc tissue, where DA uptake was increased by MP1104 (500 nM) (226 \pm 17 (mean \pm SEM); $p<0.05$ ) compared to vehicle group (157 \pm 33 ), and effects were significantly blocked after pre-incubating the tissue with nor-BNI $(1 \mu \mathrm{M})(145 \pm 77$ (mean $\pm \mathrm{SEM}) ; p<0.05)$ (Figure 3.4D). However, NTI (1 $\mu \mathrm{M})$ had no effect on MP1104 induced increases in DAT function in the dStr or NAc (Figure 3.4C \& D). One-way ANOVA with Fisher's LSD test at $p<0.05$. Effects of MP1104 in increasing DA uptake via DAT was dose dependent. 


\subsection{Anti-nociception studies}

\subsubsection{Onset and duration of action of MP1104 in warm-water tail withdrawal assay}

The warm-water tail withdrawal assay was used to assess the in vivo onset and duration of action of MP1104. Male Sprague-Dawley rats were administered a single i.p. injection of vehicle (DMSO, Tween 80 and milliQ water in a ratio 2:1:7) or morphine $(10 \mathrm{mg} / \mathrm{kg})$ or MP1104 (0.3 or $0.6 \mathrm{mg} / \mathrm{kg}$ ) and the tail withdrawal behaviour repeatedly measured over time. MP1104 showed a significant effect of treatment $\left(F_{(7,540)}=128.6, p<0.0001\right)$, time $\left(F_{(14,540)}=39.48, p<0.0001\right)$ and interaction of treatment and time $\left(F_{(42,255)}=10.68, p<0.0001\right.$, two-way repeated measures ANOVA with Bonferroni's post-test). Significant anti-nociceptive effects for MP1104 at $0.3 \mathrm{mg} / \mathrm{kg}(p<0.05)$ and $0.6 \mathrm{mg} / \mathrm{kg}(p<0.01)$ doses was shown at $45 \mathrm{~min}$ (Figure 3.5A). Morphine $(10 \mathrm{mg} / \mathrm{kg})$ treated rats also showed significant analgesic effect at $30 \mathrm{~min}(p<0.0001)$ compared to vehicle-treated rats. Anti-nociceptive effects were then evaluated in the presence of selective $\operatorname{MOPr}(\beta-F N A, 10 \mathrm{mg} / \mathrm{kg}$, s.c.), $\operatorname{KOPr}$ (nor-BNI, $10 \mathrm{mg} / \mathrm{kg}$, s.c.) and DOPr (NTI, $15 \mathrm{mg} / \mathrm{kg}$, s.c.) antagonists (Figure 3.5A) and the area under the curve (AUC) analysis calculated for each treatment group (Figure 3.5B). Morphine $(10 \mathrm{mg} / \mathrm{kg})(p<0.0001)$ and MP1104 at both $0.3 \mathrm{mg} / \mathrm{kg}(p<0.001)$ and $0.6 \mathrm{mg} / \mathrm{kg}(p<$ 0.0001) doses showed significant anti-nociceptive effects compared to vehicle controls (Figure 3.5B). Pre-treatment with the MOPr selective antagonist $\beta$-FNA, did not alter anti-nociceptive. However, pre-treatment with the selective DOPr (NTI) $(p<0.0001)$ or KOPr (nor-BNI) $(p<0.0001)$ antagonists attenuated the anti-nociceptive effects of MP1104 $(0.6 \mathrm{mg} / \mathrm{kg})$. Both nor-BNI and NTI pre-treatment fully prevented anti-nociceptive effects induced by MP1104 $(p<0.0001)$ confirming the lack of MOPr involvement in anti-nociceptive effects. 
A

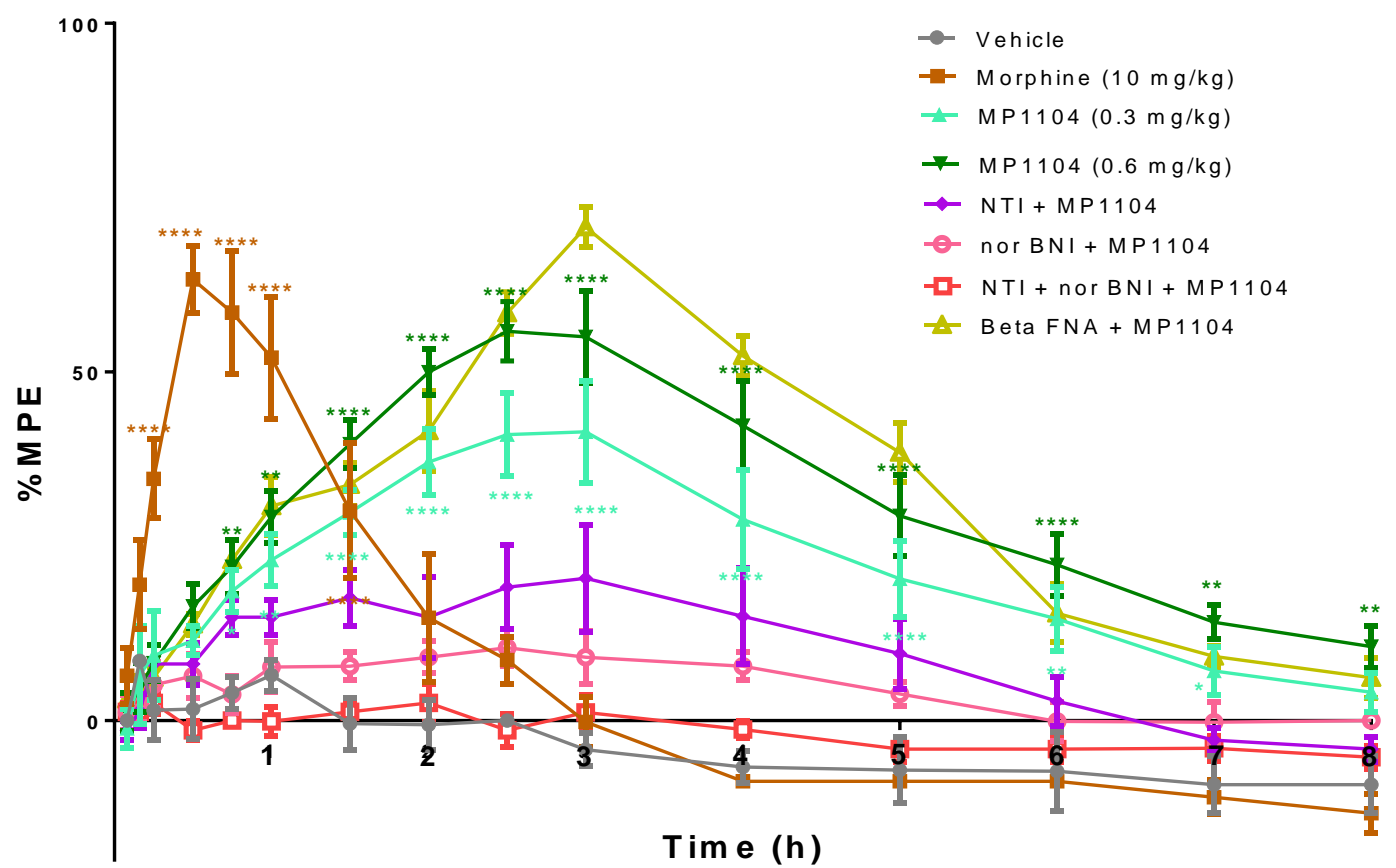

B

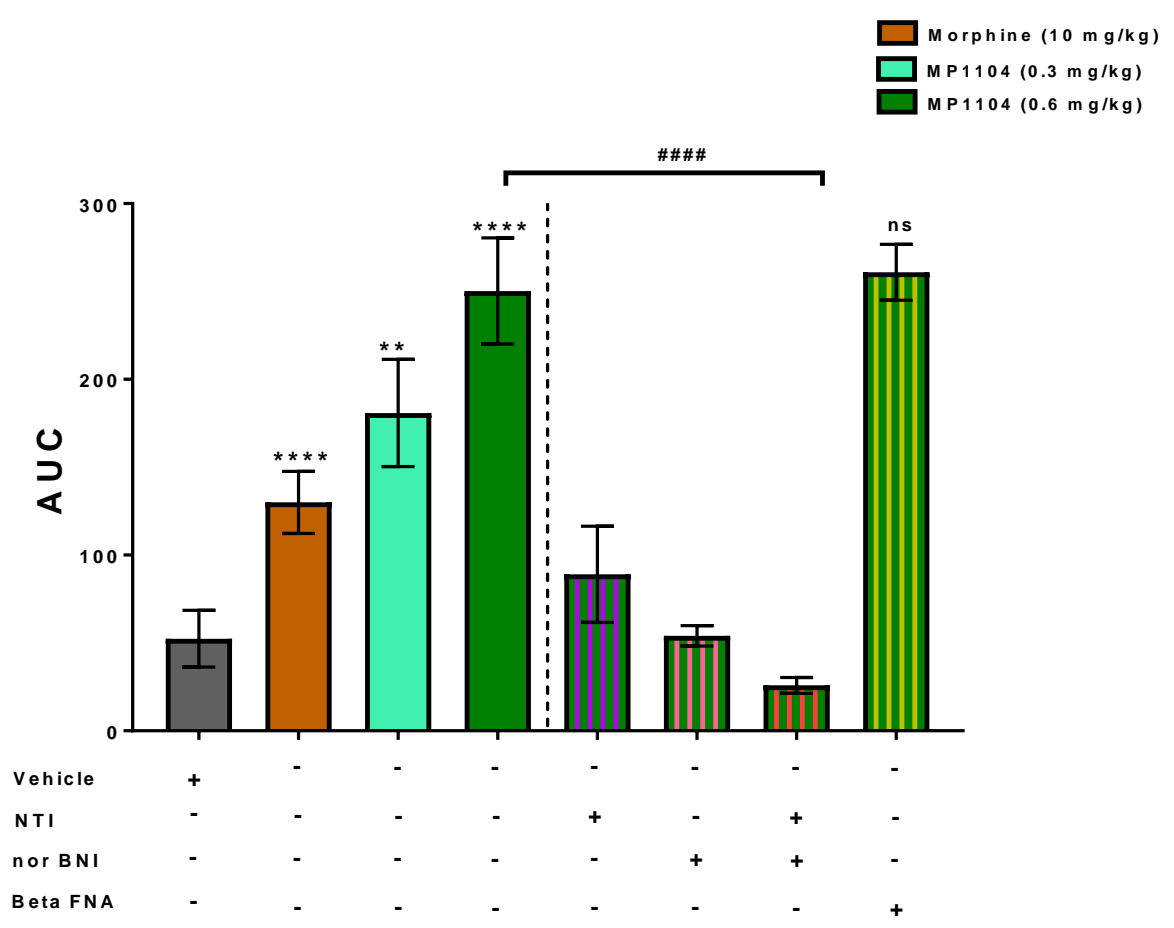


Figure 3.5: Duration of action of MP1104 in warm-water tail withdrawal assay in rats

(A) MP1104 at 0.3 and $0.6 \mathrm{mg} / \mathrm{kg}$, s.c. showed significant anti-nociception effects for 7 and $8 \mathrm{~h}$ respectively and morphine (10 $\mathrm{mg} / \mathrm{kg}$, s.c.) for up to $2 \mathrm{~h}$ (compared to vehicle) with main effect of time $\left(F_{(42,255)}=10.68, p<0.0001\right)$. (B) The area under the curve (AUC) showed MOPr antagonist, $\beta$-FNA (10 mg/kg, s.c.) had no significant (ns) effect on MP1104 $(0.6 \mathrm{mg} / \mathrm{kg})$ tail-withdrawal latencies. Nor-BNI (10 mg/kg, s.c.) and NTI (15 mg/kg, s.c.), alone significantly reduced the effects of MP1104 $(0.6 \mathrm{mg} / \mathrm{kg})$ and nor-BNI and NTI together eliminated the anti-nociceptive effects of MP1104. Two-way repeated measures ANOVA followed by Bonferroni post-tests. ${ }^{*} p<0.05,{ }^{* *} p$ $<0.01, * * * p<0.001, * * * * p<0.0001$ compared to vehicle-controls. \#\#\# $p<0.0001$ compared to MP1104 $0.6 \mathrm{mg} / \mathrm{kg}$. Values expressed as mean $\pm \mathrm{SEM}, \mathrm{n}=5-7$ per group. 


\subsubsection{Cumulative dose-response effects using the warm-water tail withdrawal assay}

MP1104 was found to be potent and efficacious in rodents. An escalating cumulative dose of MP1104 was administered to rats and mice via s.c. injections and the tail withdrawal behaviour was measured at $1 \mathrm{~h}$ intervals following each administration. Non-linear regression analysis was used to determine the efficacy $\left(E_{\max }\right)$ and potency $\left(E D_{50}\right)$. MP1104 showed $E D_{50}$ value of 0.58 $\mathrm{mg} / \mathrm{kg}$ in rats and $0.35 \mathrm{mg} / \mathrm{kg}$ mice (for $E_{\max }$ and $E D_{50}$ values see Table 3.1). All data sets passed normality testing (D’Agostino \& Pearson omnibus K2 normality test). Equivalent treatments with vehicle did not have any analgesic effect over time for both rats and mice (see Appendix D). 
A

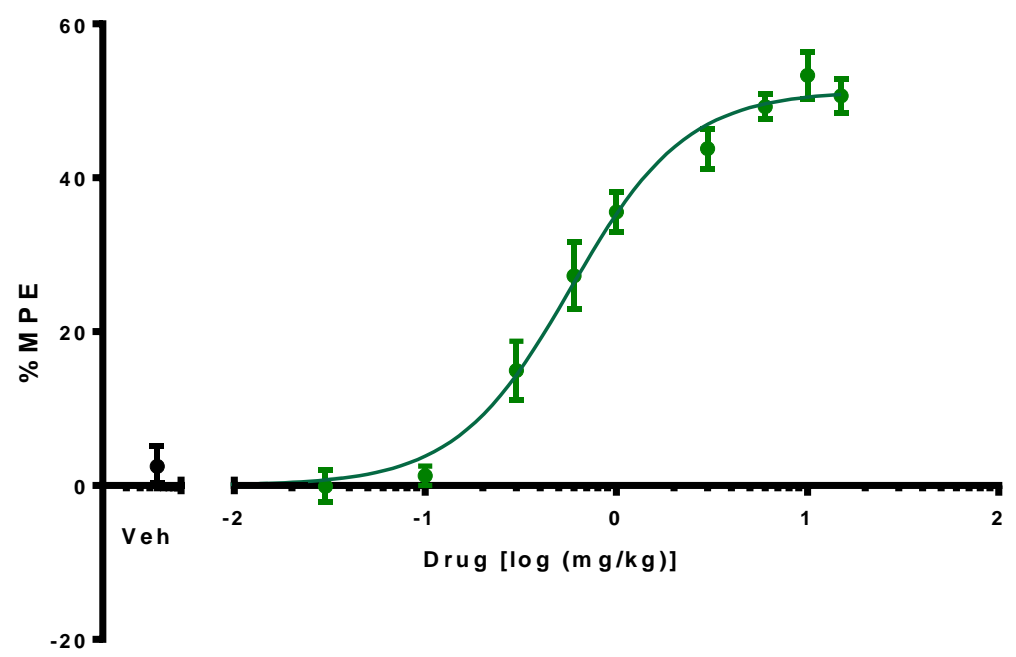

B

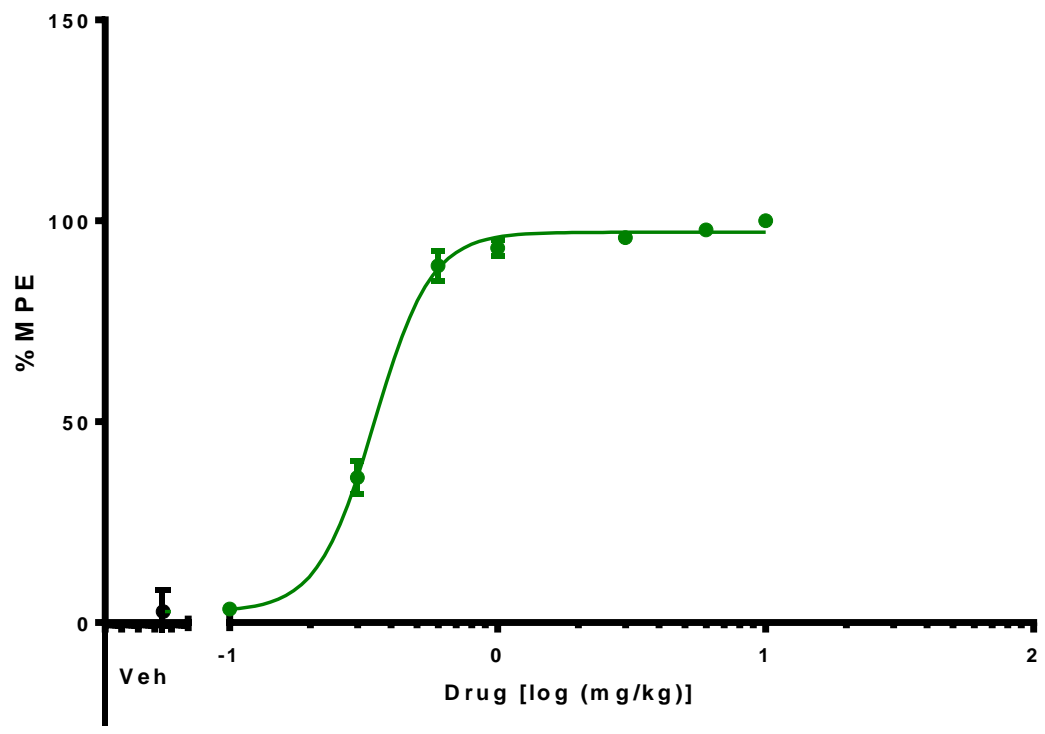

Figure 3.6: Dose-response effects of MP1104 in rats and mice using warm-water tail withdrawal assay

The cumulative dose-response effects of MP1104 was evaluated in (A) rats and (B) mice. The maximal possible effect (\%MPE) at each dose was calculated as a percent based on the pretreatment baseline latencies. Values expressed as mean $\pm S E M, n=5-6$. 
Table 3.1: Dose-response effects of MP1104 differences in rats and mice

\begin{tabular}{|l|l|l|l|}
\hline & ED $_{50}(\mathrm{mg} / \mathrm{kg})$ & $\operatorname{logED}_{50} \pm$ SEM & $E_{\max } \pm$ SEM \\
\hline Rats & 0.58 & $0.238 \pm 0.053$ & $51.31 \pm 1.877$ \\
\hline Mice & $0.35^{* *}$ & $0.249 \pm 0.012$ & $96.63 \pm 1.13$ \\
\hline
\end{tabular}

Efficacy and potency of MP1104 was evaluated in mice and rats using warm-water tail withdrawal assay. There is significant difference in potency $\left(E_{50}\right)$ between rats and mice. ${ }^{* *} p<0.01$, Student's $t$-test. Values presented as mean \pm SEM, $n=5-6$. 


\subsubsection{Cumulative dose-response effects of MP1104 on mechanical and cold allodynia}

The cumulative dose-response effects for mechanical (Figure 3.7A) and cold (Figure 3.7B) allodynia using MP1104 and morphine were evaluated in male C57BL/6 mice. Analysis of the treatment curves showed a different curve fit each data set, indicating a significant effect of treatment in mechanical $\left(\mathrm{F}_{(4,12)}=11.44, p=0.032\right)$ and cold $\left(\mathrm{F}_{(2,12)}=18.08, p=0.041\right)$ allodynia in mice. The $\mathrm{ED}_{50}$ values for MP1104 and morphine for mechanical allodynia measurements were found to be $0.449 \mathrm{mg} / \mathrm{kg}$ and $4.07 \mathrm{mg} / \mathrm{kg}$ respectively. Treatment with MP1104 showed mice had a significantly more potent $\mathrm{ED}_{50}$ value compared to morphine-treated mice ( $p=0.0386$, Student's $t$-test) (Figure 3.7A). Similarly, when measured for cold allodynia, MP1104 was found to be more potent $\left(E D_{50}=0.479 \mathrm{mg} / \mathrm{kg}\right)$ compared to morphine $\left(\mathrm{ED}_{50}=5.179 \mathrm{mg} / \mathrm{kg}\right)(p=0.0371$, Student's $t$-test) (Figure 3.7B). There were no significant differences in the baseline or day 15 measures of mechanical or cold allodynia effects when the mice were grouped dependent on the subsequent dose-response treatment, showing the mice were assigned to treatments in an unbiased manner (see Appendix F.1 and F.2). Moreover, equivalent treatments with vehicle did not have any analgesic effect over time for both measurements (see Appendix F.3). 
A

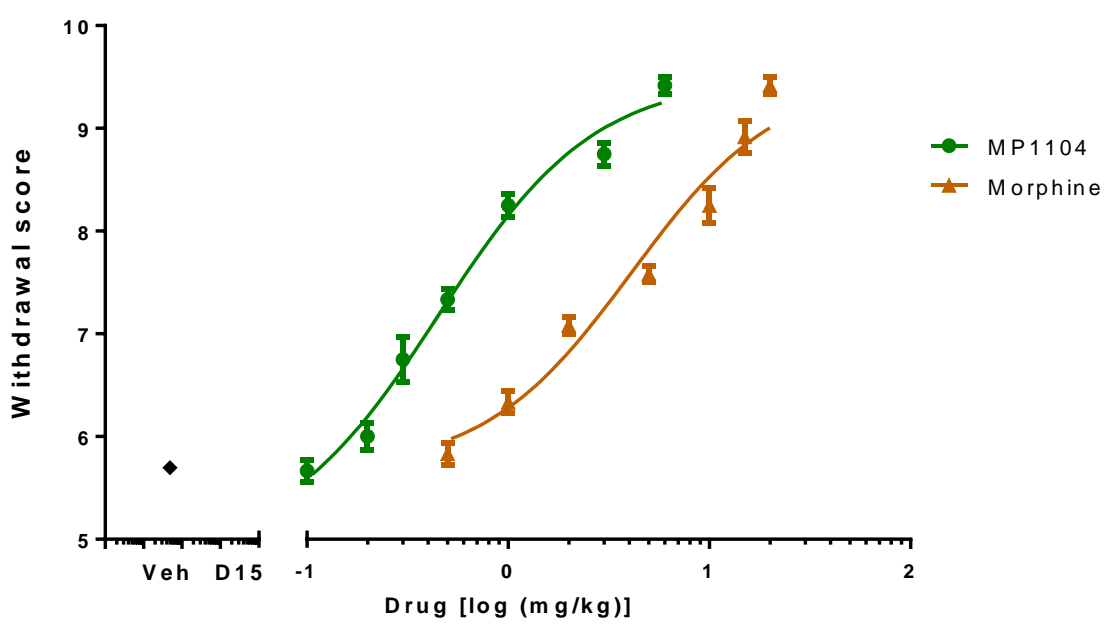

B

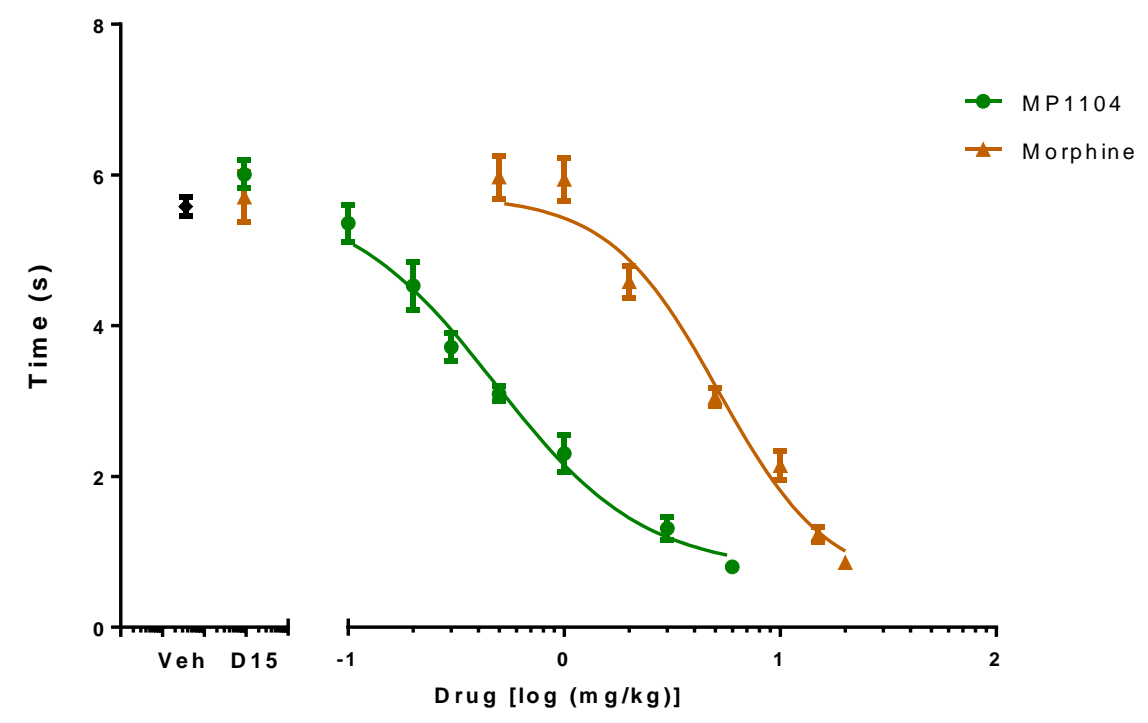

Figure 3.7: Dose-response effects of MP1104 and morphine in mice with established paclitaxelinduced neuropathic pain

The analgesic dose-response effects of morphine and MP1104 on (A) mechanical and (B) cold allodynia measures taken in mice with established paclitaxel-induced neuropathic pain. Veh: paclitaxel-treated mice injected with vehicle used as a control; D15: values on day 15 postpaclitaxel prior to the dose-response experiment. Values expressed as mean $\pm S E M, n=6$ per group. 


\subsubsection{Effect on chronic administration of MP1104 agonist on CINP-induced mechanical and cold allodynia}

Following establishment of paclitaxel-induced mechanical and cold allodynia, mice, on day 15 were divided into treatment groups so that each treatment group showed equivalent paclitaxelinduced effects in measures of both mechanical and cold allodynia (See Appendix G). Drug treatment was administered at the approximate $\mathrm{ED}_{80}$ value obtained from the acute doseresponse experiment (Figure 3.7A \&B).

\subsubsection{Mechanical allodynia}

Chronic administration of MP1104 $\left(\mathrm{ED}_{80}=1.2 \mathrm{mg} / \mathrm{kg}\right)$ reduced the paclitaxel-induced mechanical allodynia effects on all days evaluated from days $16-38(p<0.0001)$. In contrast, morphine treatment $\left(E D_{80}=10 \mathrm{mg} / \mathrm{kg}\right)$ showed significant analgesic effects on days 16-30 $(p<0.0001)$ (Figure 3.8A). Repeated measures two-way ANOVA revealed a significant effect of treatment $\left(\mathrm{F}_{(3,}\right.$ $384)=29.12, p<0.0001)$, time $\left(F_{(11,384)}=33.94, p<0.0001\right)$ and interaction of treatment and time $\left(F_{(33,384)}=27.28, p<0.0001\right)$. The main effect of treatment was compared at each time point. MP1104 reduced paclitaxel-induced mechanical allodynia effects on all days 16-38 $(p<0.0001)$, whereas morphine reduced the paclitaxel effects on day 16-30 $(p<0.05)$, after which it appears that mice became tolerant to the anti-nociceptive effects of morphine (Figure 3.8A). From the data, MP1104 shows increased potency compared to morphine. Moreover, MP1104 treatment reduced the mechanical withdrawal thresholds back to non-disease vehicle/vehicle-treated mice from day 26-38 ( $p>0.999)$, whereas morphine began to show tolerance from day 30 (Figure 3.8A). To understand the main effect of treatment, the AUC was calculated for days 16-38. AUC analysis and Bonferroni post-tests showed that both MP1104 (1.2 mg/kg, i.p., daily; $p<0.0001)$ and morphine $(10 \mathrm{mg} / \mathrm{kg}$, i.p., daily; $p<0.05)$, reduced mechanical thresholds compared to vehicle/paclitaxel treated mice (Figure $3.8 \mathrm{C}$ ). 


\subsubsection{Cold allodynia}

MP1104 $\left(E D_{80}=1.2 \mathrm{mg} / \mathrm{kg}\right)$ and morphine $\left(\mathrm{ED}_{80}=10 \mathrm{mg} / \mathrm{kg}\right)$ have shown to reduce paclitaxelinduced cold allodynia effects on all treatment days $16-38(p<0.0001)$. There was significant effect of treatment $\left(F_{(3,384)}=34.96, p<0.0001\right)$, time $\left(F_{(11,384)}=33.28, p<0.0001\right)$ and interaction of treatment and time $\left(\mathrm{F}_{(33,384)}=22.69, p<0.0001\right.$; Two-way ANOVA with Bonferroni post-tests). Bonferroni post-tests also showed a significant effect between the vehicle/vehicle negative control and the vehicle/paclitaxel treatment on days $16-38(p<0.0001)$. Morphine $(10 \mathrm{mg} / \mathrm{kg}$, i.p., daily) produced a reduction in the cold stimulus response times on days 16-24 $(p<0.05)$, whereas MP1104 (1.2 mg/kg, i.p., daily) treatment reduced cold allodynia from days 16-38 ( $p<0.0001)$. Moreover, MP1104 treatment reduced the cold response times back to the same level as the vehicle/vehicle treatment group ( $p>0.999$ ) (Figure 3.8B). The AUC analysis showed a significant effect of treatment $\left(F_{(3,24)}=19.29, p<0.0001\right)$ and treatment effects showed that MP1104, and morphine reduced the paclitaxel-induced cold allodynia effects ( $p<0.0001$; Figure 3.8D). 


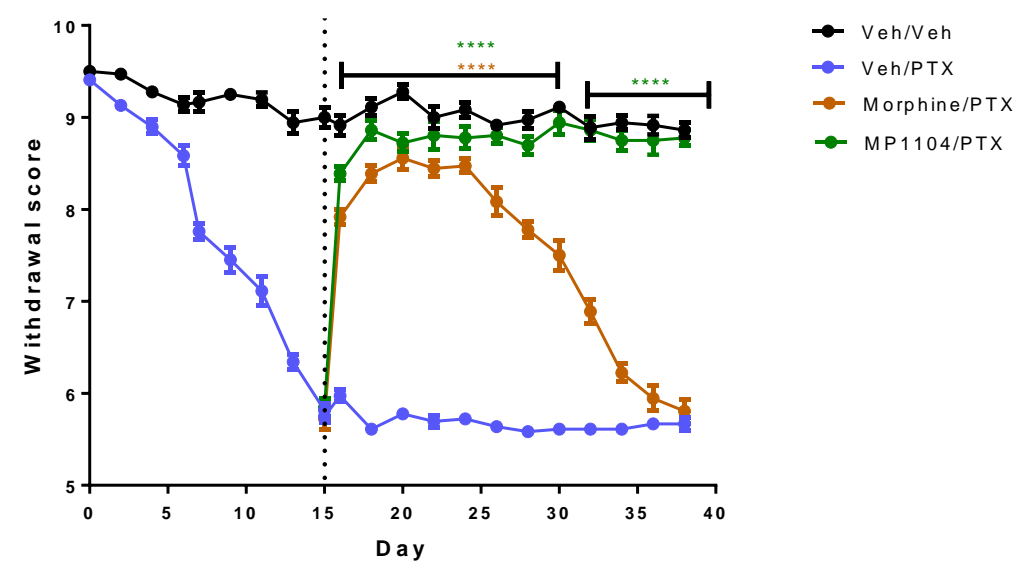

B

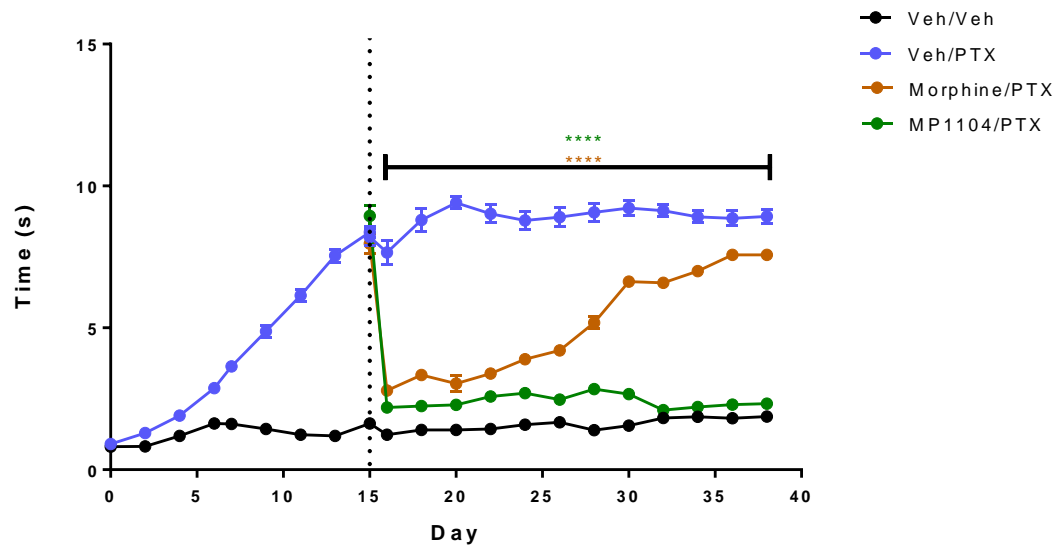




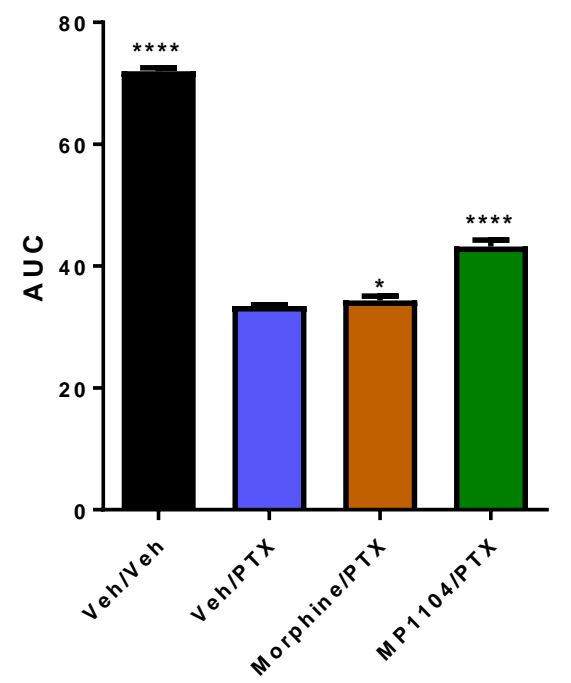

D

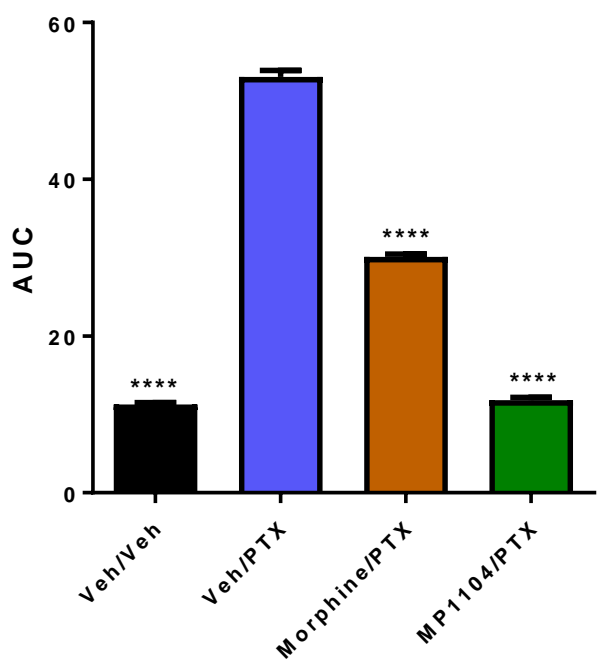

\section{Figure 3.8: Treatment of paclitaxel-induced mechanical and cold allodynia}

Time course of the treatment effects on paclitaxel-induced (A) mechanical and (B) cold allodynia. Comparison of the treatments showed morphine $(10 \mathrm{mg} / \mathrm{kg}$, i.p., daily) and MP1104 $(1.2 \mathrm{mg} / \mathrm{kg}$, i.p., daily) reduced paclitaxel-induced effects, with MP1104 reducing the mechanical threshold and cold response time to vehicle/vehicle-treated group. The area under the curve (AUC) showed morphine and MP1104 reduced (C) mechanical and (D) cold allodynia compared to vehicle/paclitaxel group. Two-way ANOVA with Bonferroni post-tests; vehicle/vehicle, MP1104 and morphine ${ }^{*} p<0.05, * * * * p<0.0001$ compared to the vehicle/paclitaxel control treatment group. Values expressed as mean \pm SEM, $n=9$ per group. 
In order to test whether the treatments were long-acting and persistent, on odd-numbered days mice were first measured for mechanical and cold allodynia prior to the administration of treatments. The results show that the effects were worn off after $24 \mathrm{~h}$ (Figure 3.9A \& B). Repeated measures two-way ANOVA was performed to understand the effects of treatment and time on mechanical withdrawal thresholds. There was significant effect of treatment $\left(F_{(3,32)}=3.96, p<\right.$ $0.0001)$, time $\left(F_{(11,352)}=5.099, p<0.0001\right)$ and interaction of treatment and time $\left(F_{(33,384)}=3.51\right.$; $p<0.0001$; Two-way ANOVA with Bonferroni post-tests). MP1104 showed significant effects on days 17-29 (Figure 3.9A). There was significant improvement between the vehicle/paclitaxel group and MP1104 ( $p<0.001)$ as well as morphine treatment $(p<0.05$; Figure $3.9 C)$.

Similarly, a two-way ANOVA performed on the response times to a cold acetone stimulus showed a significant effect of treatment $\left(\mathrm{F}_{(3,384)}=2.71, p<0.0001\right)$, time $\left(\mathrm{F}_{(11,384)}=5.287, p<0.0001\right)$ and interaction between treatment and time $\left(\mathrm{F}_{(33,384)}=1.934 ; p=0.0019\right)$. MP1104 showed significant effects on days 17-27, whereas morphine showed significant effects on days 19-21 (Figure 3.9B). The results showed no significant improvement of MP1104 or morphine treatment on cold allodynia compared to vehicle/paclitaxel group (Figure 3.9D). 
A

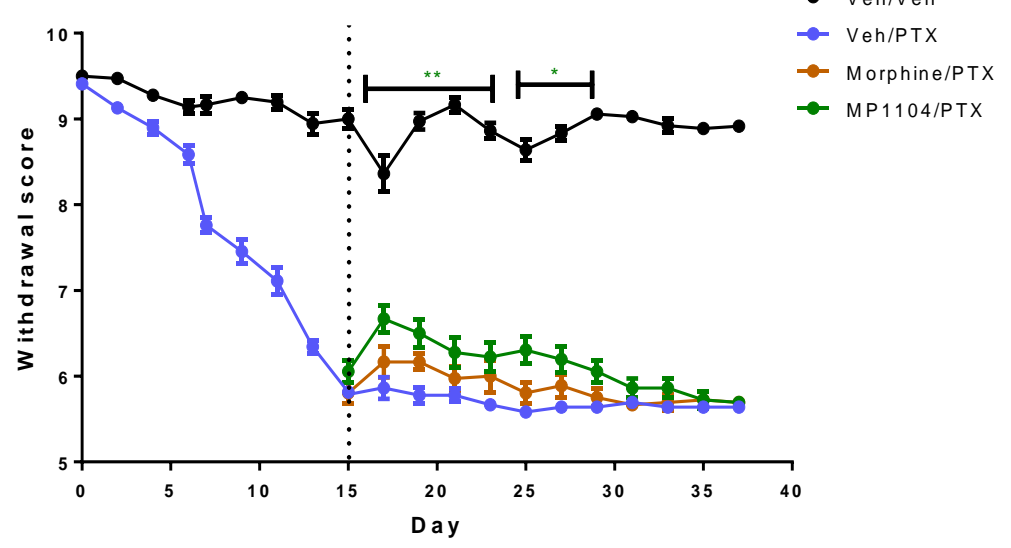

B

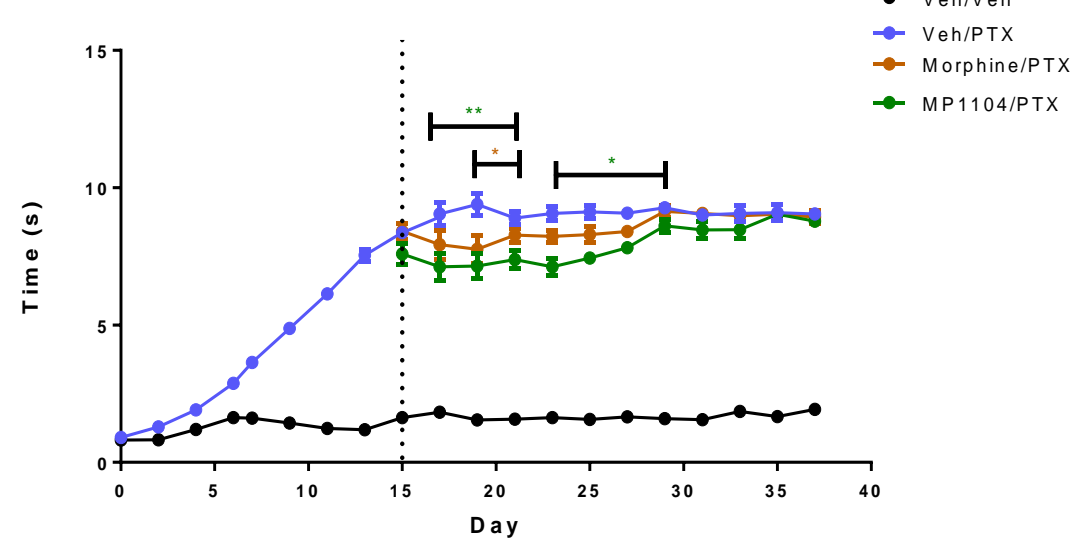


C

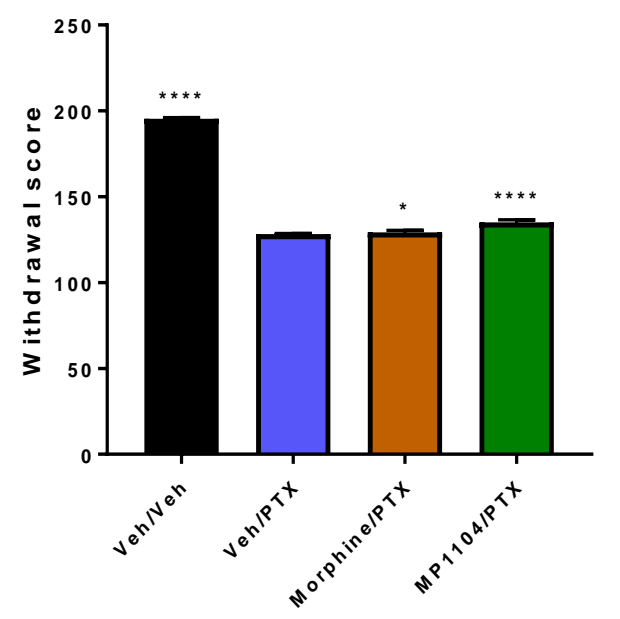

D

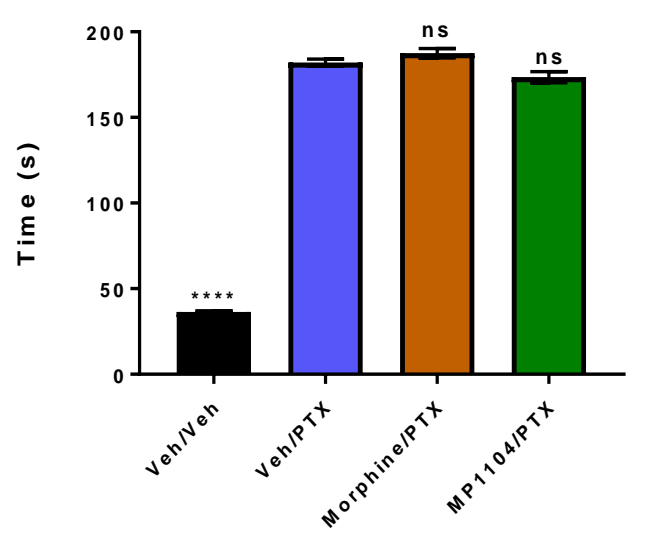

Figure 3.9: Mechanical and cold allodynia with measurements before daily treatment

Time course of the anti-nociceptive effects of MP1104 and morphine on paclitaxel-induced (A) mechanical and (B) cold allodynia 24 hours after administration of the treatments. (C) The area under the curve (AUC) showed morphine and MP1104 significantly reduced mechanical allodynia compared to vehicle/paclitaxel group. (D) No significant (ns) improvement on cold allodynia was observed in morphine or MP1104 treatments compared to vehicle/paclitaxel group. Two-way ANOVA with Bonferroni post-tests. ${ }^{*} p<0.05, * * p<0.01, * * * * p<0.0001$ compared to the vehicle/paclitaxel control treatment group. Values expressed as mean \pm SEM, $n=9$ per group. 


\subsection{Side effects studies}

\subsubsection{Effect of MP1104 on sedation}

MP1104, a mixed KOPr/DOPr agonist was evaluated for sedative property using spontaneous locomotor activity test in male Sprague-Dawley rats.

The time course analysis of spontaneous locomotor activity for total ambulatory and stereotypic counts showed a non-significant interaction between time and MP1104 administration $\left(F_{(3,72)}=\right.$ $0.07611, p=0.9727)$ and $\left(F_{(3,72)}=0.2116, p=0.888\right)$ respectively (see Figure $\left.3.10 \mathrm{~A} \& \mathrm{~B}\right)$. There was significant effect in time in both total ambulatory $\left(\mathrm{F}_{(18,126)}=116.9, p<0.0001\right)$ and stereotypic counts $\left(F_{(18,126)}=91.72, p<0.0001\right)$, however a non-significant effect of treatment for both total ambulatory $\left(\mathrm{F}_{(3,21)}=0.511, p=0.678\right)$ and stereotypic counts $\left(\mathrm{F}_{(3,21)}=0.966, p=0.426\right)$. MP1104 produced an average ambulatory count of $459 \pm 173,259 \pm 172$ and $123 \pm 154$ at 0.3, 0.6 and 1 $\mathrm{mg} / \mathrm{kg}$ respectively during the 60 min testing phase, whereas vehicle-treated rats produced a mean of $704 \pm 136$ ambulatory counts (Figure 3.10E). In addition, the average stereotypic counts for MP1104 treated rats was found to be $2127 \pm 1053,1637 \pm 1543$ and $995 \pm 634$ at $0.3,0.6$ and $1 \mathrm{mg} / \mathrm{kg}$ respectively vs vehicle-treated rats with $3180 \pm 688$ stereotypic counts. AUC analysis showed that MP1104 at $0.3 \mathrm{mg} / \mathrm{kg}(p=0.2044)$ and $0.6 \mathrm{mg} / \mathrm{kg}(p=0.1642)$ did not significantly alter ambulatory counts in the $60 \mathrm{~min}$ locomotor activity test (Figure 3.10E). Similarly, at 0.3 and $0.6 \mathrm{mg} / \mathrm{kg}$ MP1104 did not alter stereotypic counts also (Figure 3.10F). However, a non-significant trend was seen at $0.6 \mathrm{mg} / \mathrm{kg}$ in both locomotor measures. At $1 \mathrm{mg} / \mathrm{kg}, \mathrm{MP} 1104$ showed significant sedative effects in both ambulatory and stereotypic counts when compared to vehicle-treated control rats $(p<0.05$; Figure $3.10 \mathrm{E} \& \mathrm{~F})$. Analysis of the $30 \mathrm{~min}$ habituation phase showed that rats in the vehicle group had no significant difference in ambulatory or stereotypic counts compared to rats treated with different doses of MP1104 (One-way ANOVA; Figure 3.10C \&D). 
A

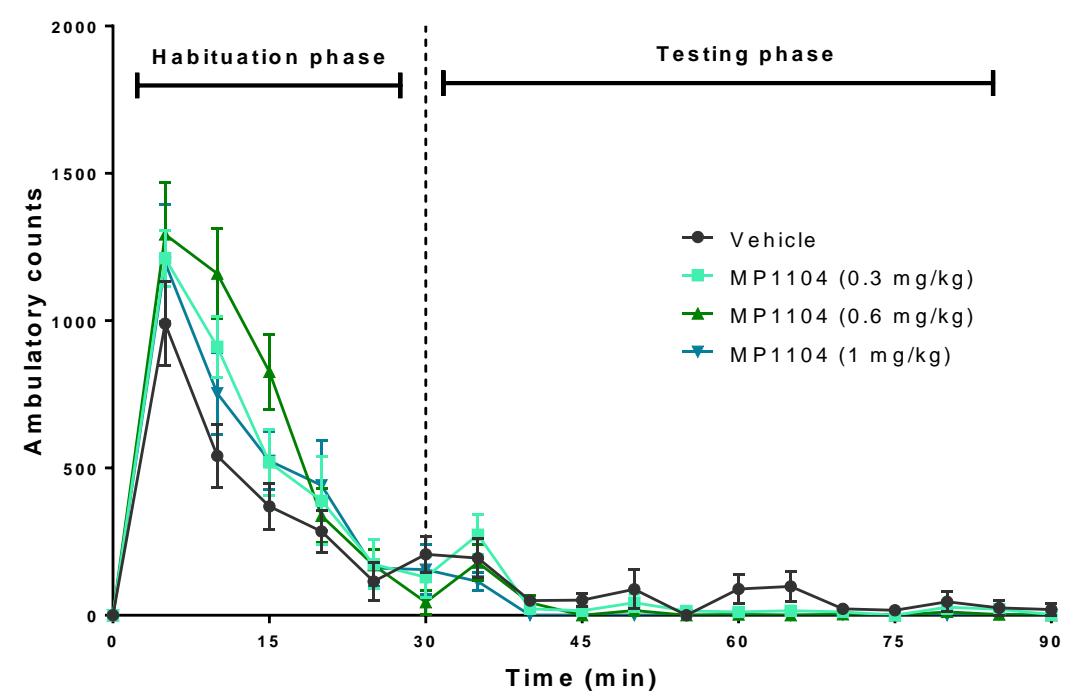

B

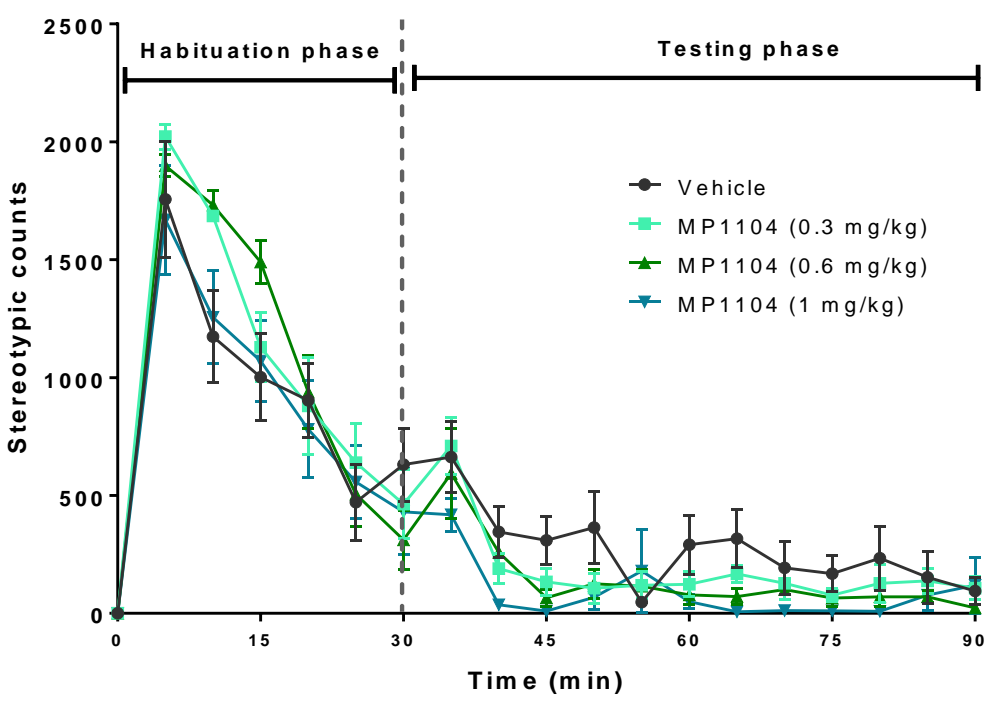


C

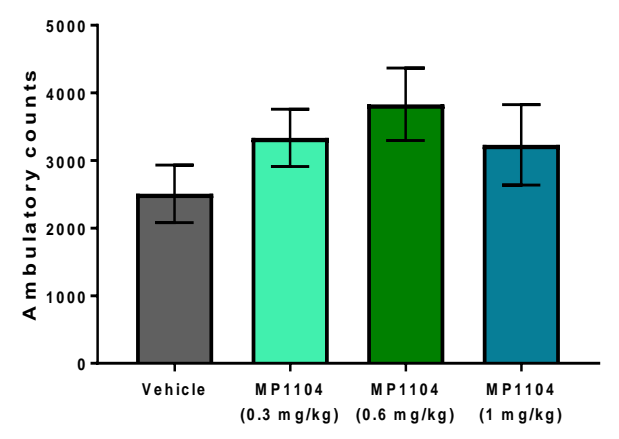

Testing phase

E

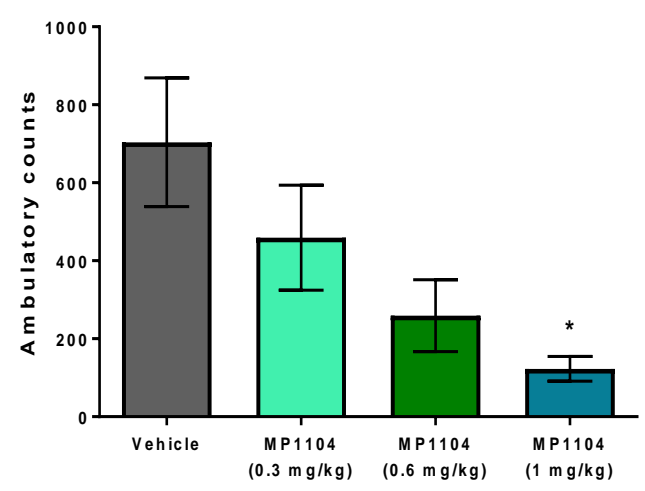

D

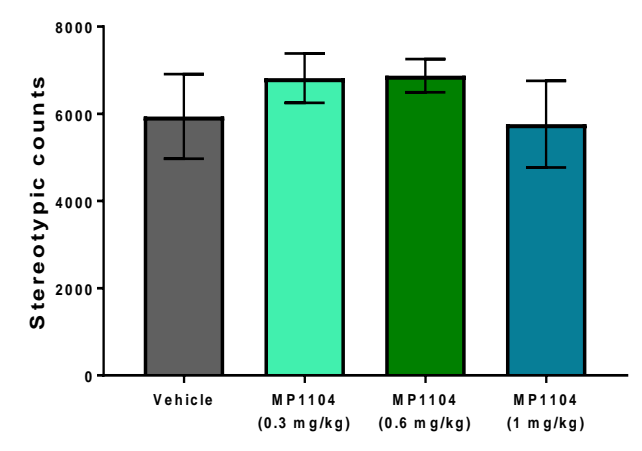

$\mathbf{F}$

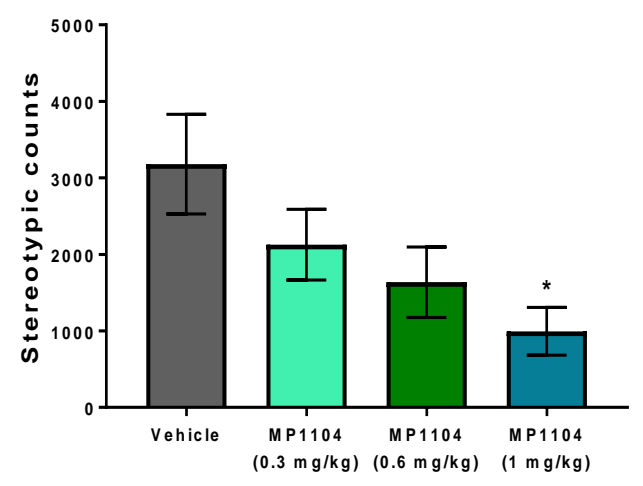

\section{Figure 3.10: Effects of MP1104 on locomotor activity}

MP1104 (0.3, 0.6 or $1 \mathrm{mg} / \mathrm{kg}$, i.p.) treated rats showed no significant changes in (A) ambulatory and (B) stereotypic counts compared to vehicle controls (Two-way ANOVA with Bonferroni's posttest). (C) Total ambulatory and (D) stereotypic counts recorded during habituation phase (30 $\mathrm{min}$ ) showed no significant change in both measures between vehicle and MP1104 0.3, 0.6 and $1 \mathrm{mg} / \mathrm{kg}$ groups. (E) Total ambulatory and (F) stereotypic counts during testing phase (60 $\mathrm{min}$ ) showed non-significant difference between MP1104 at 0.3 and $0.6 \mathrm{mg} / \mathrm{kg}$ compared to vehicle control, but at higher dose MP1104 (1 m/kg) was found to be significantly sedative compared to vehicle control group. One-way ANOVA, ${ }^{*} p<0.05$ compared to vehicle group. Values presented as mean $\pm \mathrm{SEM}, \mathrm{n}=8$ per group. 


\subsubsection{Effect of MP1104 on aversion}

Previously it was shown that pure KOPr agonist such as Sal A and U50,488 produce aversion in CPA tests (see Table 4.1). In this study, we measured the aversive effects of MP1104 (0.3 and 0.6 $\mathrm{mg} / \mathrm{kg}$ ) at doses that did not produce sedative effects.

Consistent with previous findings, U50,488 (10 mg/kg, i.p.) significantly reduced the time spent by rats in the paired chamber by $23 \%$ (pre-test of $53 \pm 6 \%$ vs. post-test of $41 \pm 2 \%$; $\left(t_{(6)}=0.586\right) ; p$ $<0.01$, Student's t-test) (Figure 3.11A). Whereas, MP1104 (0.6 mg/kg, i.p.) produced no significant changes in time spent in the paired chamber $\left(\left(t_{(6)}=4.63\right), p=1.385\right.$, Student's $t$-test $)$. Also, the vehicle-treated rats (DMSO:Tween-80:MilliQ water in a 2:1:7 ratio) did not produce any significant changes in percentage of time spent in the paired chamber, with only $8 \%$ increase recorded (28 $\pm 8 \%$ before conditioning vs $31 \pm 4 \%$ after conditioning). To test whether the DOPr activity of MP1104 was responsible for negating the aversive-like effects we blocked the DOPr agonist activity of MP1104 using NTI (15 mg/kg, s.c.). Upon blockade with NTI, MP1104 showed significantly less time in the paired chamber (pre-test of $58 \pm 7 \%$ vs. post-test of $45 \pm 3 \%$; $p<0.05$, Student's $t$-test), indicating that the rewarding properties of DOPr agonism counters the aversivelike side effects of KOPr agonism. Using CPA model we also analysed the total distance travelled by the animal, an additional supporting method to evaluate motor behaviours. Although no significant difference was observed between MP1104 $(0.3 \& 0.6 \mathrm{mg} / \mathrm{kg})$ and vehicle $\left(F_{(9,64)}=2.69\right.$, $p=0.214)$; One-way ANOVA), the trend in the data shows that MP1104 treated rats did not show reduced locomotor movements compared to vehicle-treated rats (Figure 3.11B). Five rats were excluded from CPA test (three rats showed $>80 \%$ preference for a chamber and two rats showed $>40 \%$ preference for the corridor). 
A

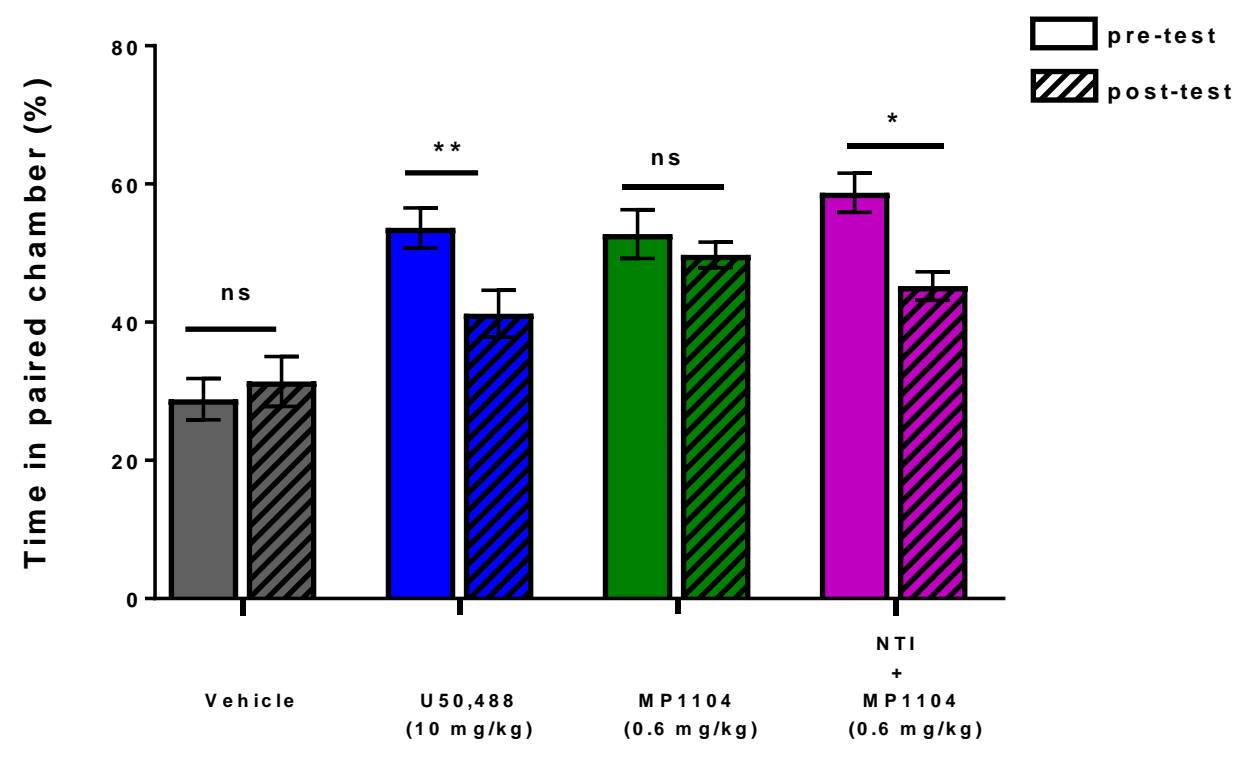

B

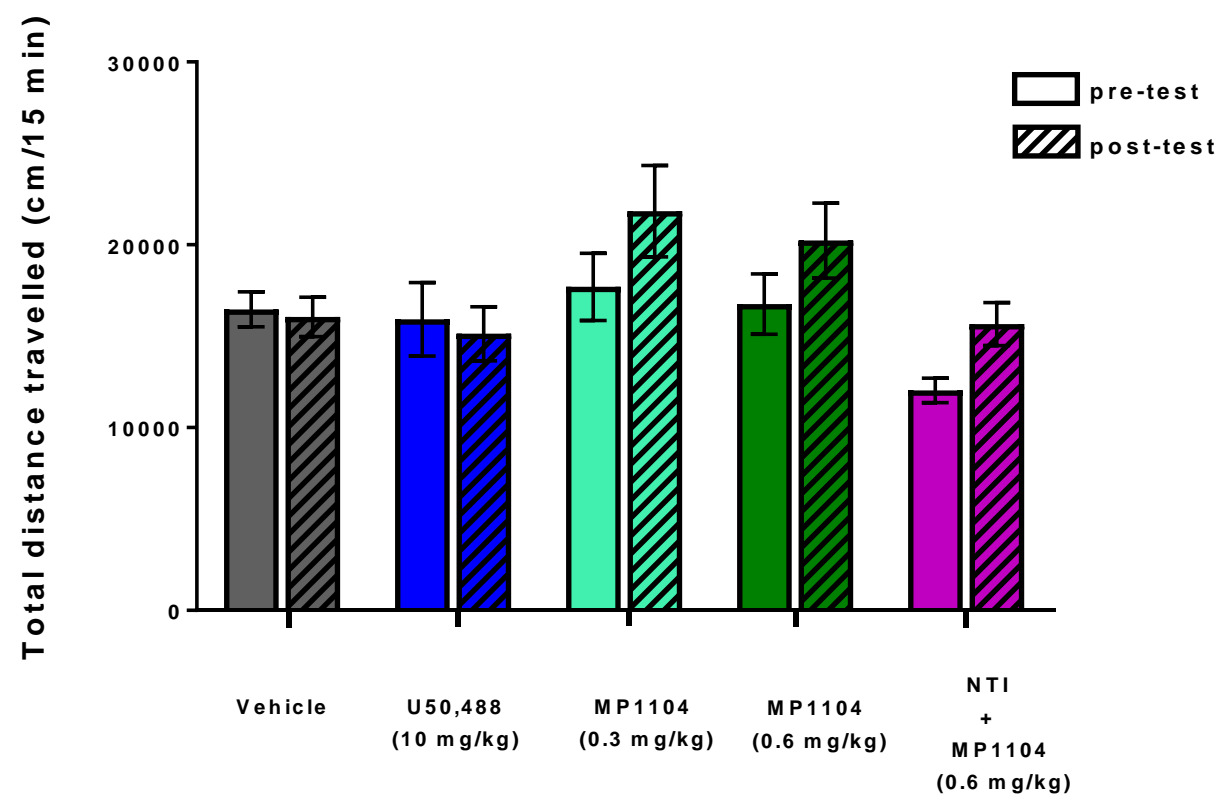




\section{C}

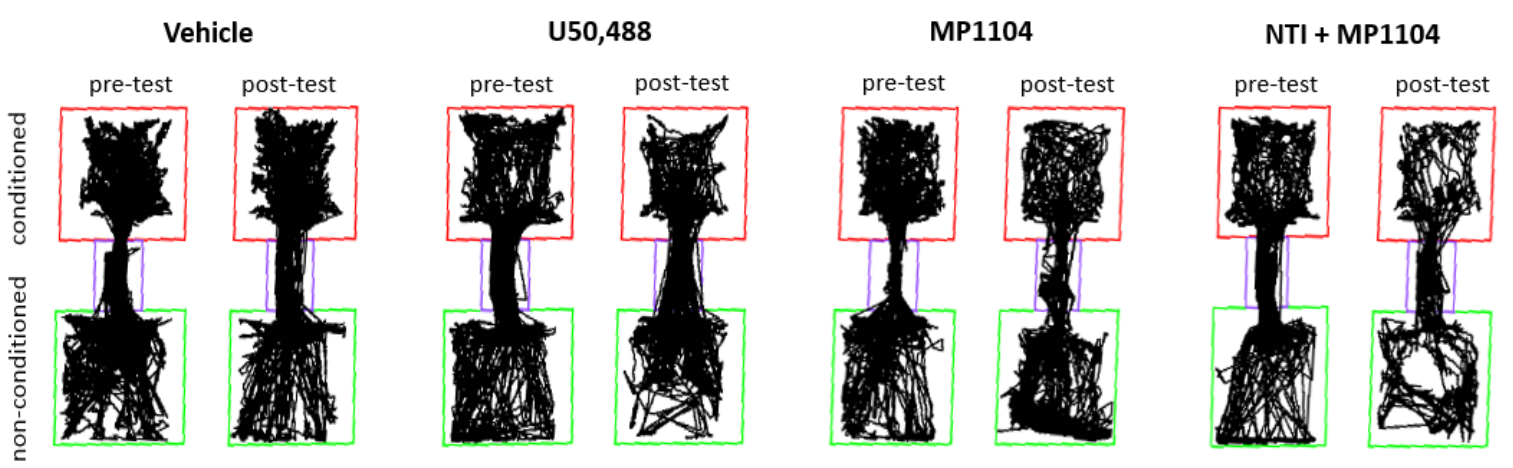

\section{Figure 3.11: Effects of MP1104 on aversion}

(A) Acute administration of MP1104 $(0.6 \mathrm{mg} / \mathrm{kg}$, i.p.) showed no aversive effects in CPA tests, however, significant aversion with MP1104 is seen following pre-treatment with NTI $(15 \mathrm{mg} / \mathrm{kg}$, s.c.) and the traditional KOPr agonist U50,488 (10 mg/ $/ \mathrm{kg}$, i.p). (B) Total distance travelled in CPA test showed no significant changes in locomotion following MP1104 (0.3 \& $0.6 \mathrm{mg} / \mathrm{kg}$, i.p.) administration in rats. Paired Student's $t$-test ${ }^{*} p<0.05,{ }^{* *} p<0.01$ compared to vehicle controls. (C) Representation of trajectory traces indicates the exploratory behaviour of rats in the pre- and post-conditioning tests. Values presented as mean $\pm S E M, n=6-7$ per group. 


\subsubsection{Effect of MP1104 on anxiety}

The anxiogenic behavioural effects of MP1104 were explored using EPM test in rats. MP1104 (0.3 and $0.6 \mathrm{mg} / \mathrm{kg}$ ) showed no significant changes in time spent in the open arm compared to the vehicle control $\left(\left(\mathrm{F}_{(3,42)}=2.141\right) ; p>0.9999\right.$, One-way ANOVA followed by Bonferroni's post-test; Figure 3.12A). MP1104 treated rats showed a non-significant increase in time spent to explore the open arm of EPM by $13 \%$ at $0.3 \mathrm{mg} / \mathrm{kg}(125 \pm 4 \mathrm{~s})$ and $1 \%$ at $0.6 \mathrm{mg} / \mathrm{kg}$ (107 $\pm 1 \mathrm{~s})$ compared to vehicle control (108 $\pm 6 \mathrm{~s})$. However, Yohimbine $(2.5 \mathrm{mg} / \mathrm{kg})$ showed a significant $40 \%$ reduction of time spent in the open arm compared to vehicle control (65 $\pm 3 \mathrm{~s} ; p=0.026$ ). Further analysis of the total arm (closed and open) entries showed that MP1104 $(0.3 \& 0.6 \mathrm{mg} / \mathrm{kg})$ treated rats produced a non-significant change in locomotion behaviour compared to vehicle, however yohimbine treated rats significantly reduced the entries into the arms $(p<0.05$; One-way ANOVA; Figure 3.12B). All treatment injections given via i.p. route of administration. 
A

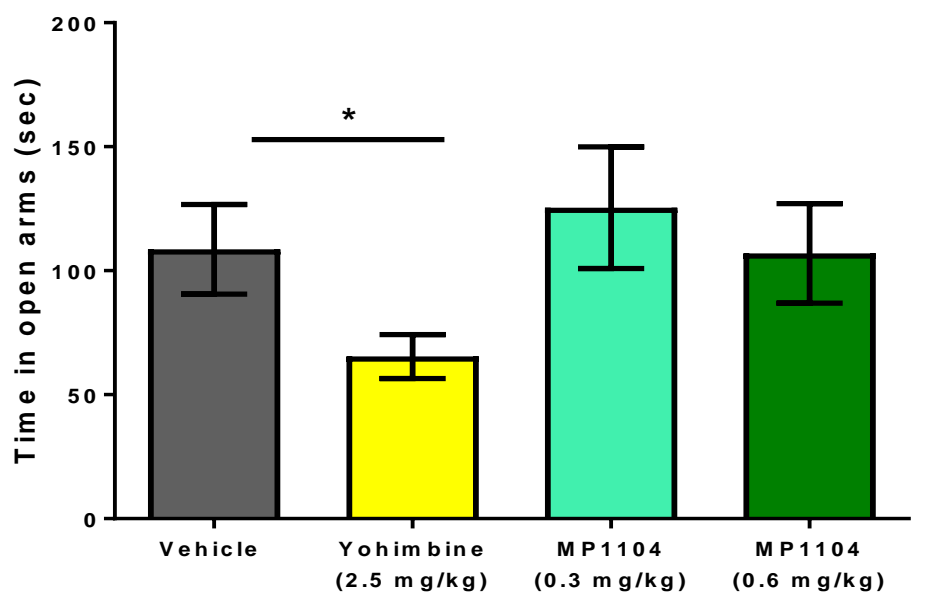

B

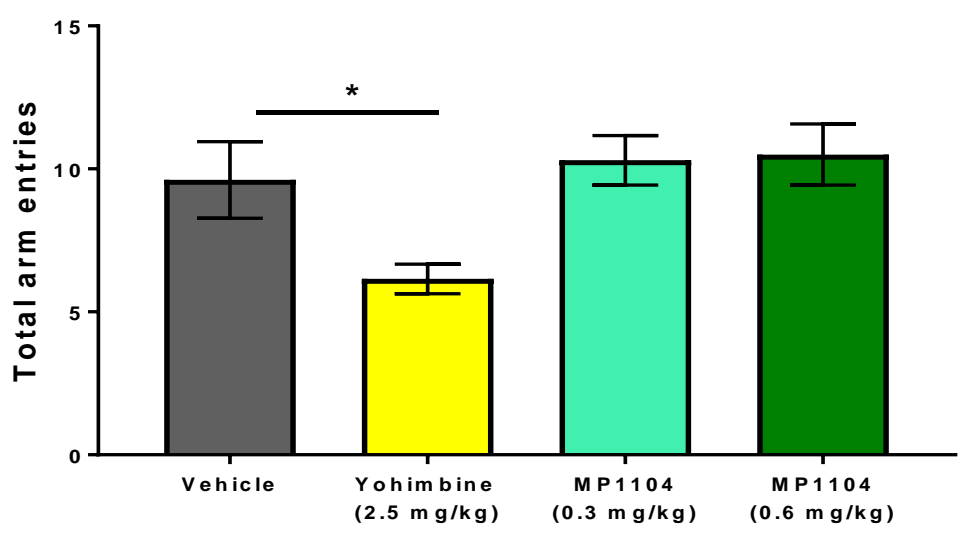

\section{Figure 3.12: Effects of MP1104 on anxiety}

Rats pre-treated with MP1104 (0.3 and $0.6 \mathrm{mg} / \mathrm{kg})$ showed no significant effects in (A) open arm times or (B) the total number of arm entries in EPM test compared to vehicle controls (DMSO:Tween-80:MilliQ water in a 2:1:7 ratio). However, the known anxiogenic drug yohimbine $(2.5 \mathrm{mg} / \mathrm{kg}$, i.p.) showed significant decrease in both time spent in the open arm and closed arm entries. One-way ANOVA; ${ }^{*} p<0.05$ compared to vehicle. Values presented as mean \pm SEM, $\mathrm{n}=$ 10-13 per group. 


\subsubsection{Effect of MP1104 on depression}

Selective KOPr administration has reported to show depressive-like behaviours in rats (Mague et al., 2003). Here we screened MP1104 for its effects on pro-depression using FST in male SpragueDawley rats. Rats treated with MP1104 at 0.3 or $0.6 \mathrm{mg} / \mathrm{kg}$ did not show any significant differences between the amounts of time rats were scored as displaying immobile, swimming and climbing behaviours compared to vehicle control $(p>0.9999$; two-way ANOVA followed by Bonferroni's post-test; Figure 3.13A). MP1104 treated rats showed no changes in mobility (swimming and climbing) or immobility compared to vehicle-treated rats ( $p>0.9999$; One-way ANOVA followed by Bonferroni's post-test; Figure 3.13B). There was significant effects of treatment $\left(F_{(5,38)}=18.42\right) ; p<0.001$, One-way ANOVA followed by Bonferroni's post-test $)$. MP1104 showed no pro-depressive effects in FST in rats. 
A

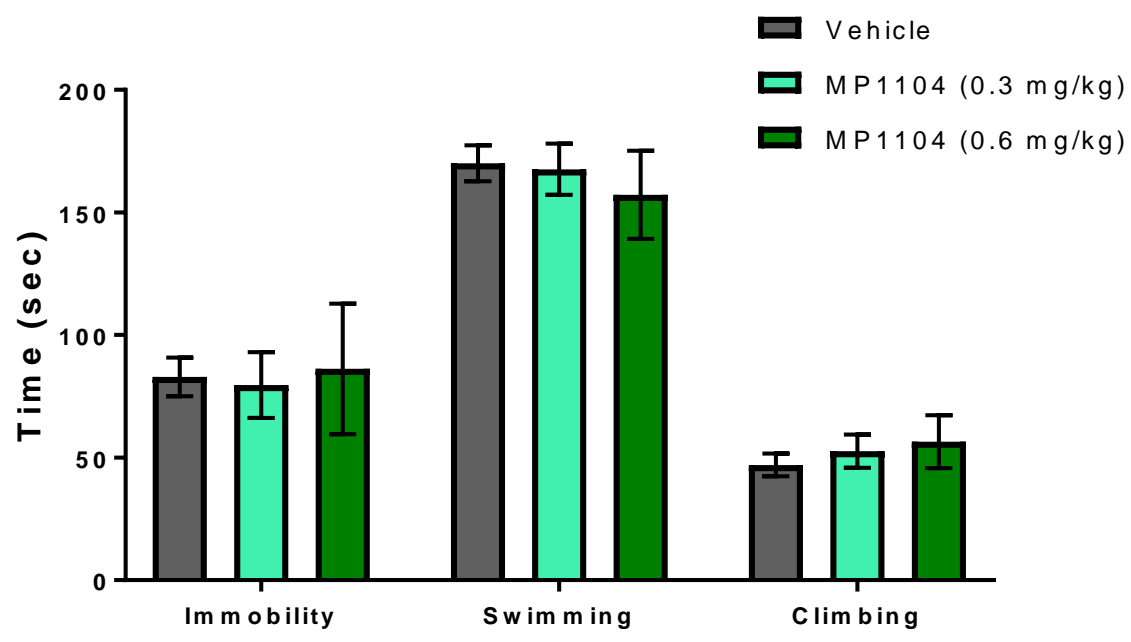

Activity

B

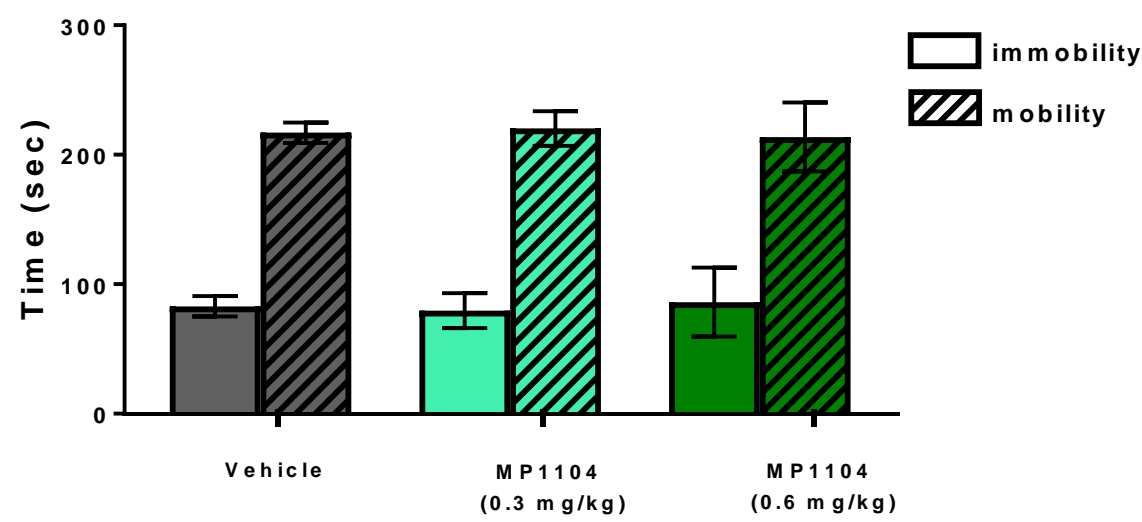

\section{Figure 3.13: Effects of MP1104 on depression}

(A) In FST, rats pre-treated with MP1104 (0.3 and $0.6 \mathrm{mg} / \mathrm{kg})$ displayed no significant change in immobility, swimming and climbing time compared to vehicle controls (two-way ANOVA). (B) Combining the swimming and climbing behavioural time observed as mobility time, MP1104 at 0.3 and $0.6 \mathrm{mg} / \mathrm{kg}$ showed no changes in mobility or immobility compared to vehicle treated controls. One-way ANOVA followed by Bonferroni's post-test, $n=7-8$ per group. All data expressed as mean \pm SEM. 


\section{Chapter 4: Discussion}

\subsection{Anti-cocaine effects}

This study evaluated the anti-cocaine effects of the mixed opioid agonist, MP1104 in male Sprague-Dawley rats. Specifically, to assess whether the mixed opioid agonist activity of MP1104 was capable of modulating cocaine taking and cocaine seeking behaviours in rats using cocaine self-administration models.

\subsubsection{Effect of MP1104 on cocaine-induced drug seeking and drug taking}

As drug self-administration models are considered the gold standard for assessing the effects of drugs of abuse in animals (Carter and Griffiths, 2009; Katz and Higgins, 2003) (see section 1.2.5 for details), we used cocaine-primed drug reinstatement tests to evaluate cocaine-induced drug seeking and dose-response cocaine self-administration tests to assess drug taking behaviour in rats.

In the cocaine-primed reinstatement model, rats achieved acquisition criteria within 7 days of cocaine self-administration on FR1 (Figure 3.1A). We found that animals reinstated cocaineseeking behaviour when exposed to a priming injection of cocaine $(20 \mathrm{mg} / \mathrm{kg}$, i.p.). Because the light stimulus is removed during extinction, and present during reinstatement, reinstatements tests measure a combination of both drug and light cue responding in this model. A within-subject Latin square design was used to minimise treatment sequence bias and the number of animals used. Rats were subjected to 6 sessions of the reinstatement cycle to complete all the drug treatments (Appendix C.1).

The drug-primed reinstatement models can be conducted as a within-session, between-session or between-within session model. In the within-session model, all three phases (stable FR5 selfadministration, extinction and reinstatement) are performed on the same day (De Wit and Stewart, 1981), during which animals are allowed to self-administer for $1-2 \mathrm{~h}$, followed by $3-4$ h extinction phase preceding a reinstatement test. This model however does not take into account the neuroadaptations associated with long-term withdrawal from the drug self-administration. Hence, we did not use this model. The between-session model is performed over successive everyday sessions (Stretch et al., 1971). Whereas, the between-within method is performed over several days, and extinction and reinstatement phases being performed on the same day (Tran- 
Nguyen et al., 1998). In this study, we utilised the between-session procedure, as this model helps in better determination of the modulating effects of KOPr system on long-term withdrawal from cocaine self-administration. Using this model we evaluated the ability of MP1104 to attenuate cocaine-induced reinstatement of drug seeking behaviour using the methods previously described in rats (Ewald et al., 2017; Riley et al., 2014). Rats were used to measure anti-cocaine effects as previously studies have shown the endogenous reward system to be similar in human and rats Deroche-Gamonet et al., 2004; Pickens et al., 2011).

Our data shows that MP1104 (0.3 and $1 \mathrm{mg} / \mathrm{kg}$ ) dose-dependently reduced cocaine-primed reinstatement of drug seeking behaviour following extinction, an effect that was found to be KOPr mediated (Figure 3.2) (also refer to Atigari et al., 2019). Previously MP1104 at $1 \mathrm{mg} / \mathrm{kg}$ has been shown to block cocaine CPP in mice, another model that assesses drug addiction behaviour in animals (Varadi et al., 2015). Hence, we chose $1 \mathrm{mg} / \mathrm{kg}$ as the highest dose in cocaine-primed reinstatement of drug seeking behaviour in rats. However, at this dose MP1104 was found to cause sedative effects in rats (see Figure 3.10), we then changed the dose to $0.6 \mathrm{mg} / \mathrm{kg}$ as highest dose in subsequent studies. Previous studies using selective $\mathrm{KOPr}$ agonists were shown to attenuate the rewarding properties of drugs of abuse in this same model (Kivell et al., 2014b; Schenk et al., 1999). KOPr agonists such as U50,488 (30 mg/kg, i.p.), Sal A (0.3 and $1 \mathrm{mg} / \mathrm{kg}$, i.p.) and U69,593 (0.3 and $0.32 \mathrm{mg} . \mathrm{kg}$, s.c.) were shown to reduce cocaine-induced drug seeking in rats (Morani et al., 2009; Schenk et al., 1999, 2000) (see Table 1.1). MP1104 attenuated cocaineinduced drug seeking in rats at similar doses to both Sal A and U69,593, consistent with its potent KOPr effects in vitro.

DOPr agonists are known to increase the rewarding properties of psychostimulants (Pradhan et al., 2011). Therefore, this study also evaluated whether the mixed actions of MP1104 would be effective in modulating drug seeking behaviours in rats. This study found that the DOPr agonist properties of MP1104 did not negate the KOPr mediated attenuation of drug seeking behaviours. This is likely due to the higher potency of MP1104 in activating $\operatorname{KOPr}\left(E D_{50}=0.027 \pm 0.002 n M\right)$ compared to $\mathrm{DOPr}\left(\mathrm{ED}_{50}=0.41 \pm 0.11 \mathrm{nM}\right)$ as observed using $\left[{ }^{35} \mathrm{~S}\right] \mathrm{GTP} \gamma \mathrm{S}$ functional assay using membranes prepared in CHO cells expressing mouse MOPr, KOPr or DOPr (Varadi et al., 2015) (see Table 1.3).

Despite certain limitations, the drug-prime reinstatement model has good face validity because conditions such as stress, drug cues etc., reliably reinstate drug seeking both in laboratory animals 
and in humans (Self and Nestler, 1998; Shalev et al., 2002; Stewart, 2000) (see section 1.2.5 for details). However, it has been argued, that the reinstatement model may not imitate most situations in humans that leads to drug abstinence (Marlatt, 1996). It has been reasoned that the effect of priming drug injections in the reinstatement model may not be relevant to drug addiction (Everitt and Robbins, 2000). The model is limited because of its dependence on experimenterdelivered triggers to induce craving in subjects (Katz and Higgins, 2003) and during the drugreinstatement phase, responses are not reinforced, whereas drug seeking in humans results in intake of drug (Shaham et al., 2003).

Modulation of the KOPr system, via both agonists and antagonists, have the potential to modulate different stages of addiction. KOPr antagonists have been investigated as potential treatments for preventing stress-induced relapse (Bruchas et al., 2010; Redila and Chavkin, 2008). Whereas, KOPr agonists have anti-cocaine properties in preclinical models of drug use and have been shown to attenuate the rewarding effects of psychostimulants (Morani et al., 2009; Shippenberg et al., 2007; Simonson et al., 2015), ethanol (Lindholm et al., 2001; Logrip et al., 2009), heroin (Zhou et al., 2013), and morphine (Glick et al., 1995; Kuzmin et al., 1997). Novel KOPr agonists such as Sal A (0.3 and $1 \mathrm{mg} / \mathrm{kg}$, i.p.) (Morani et al., 2009), methoxymethyl ether Salvinorin B (0.3 mg/kg, i.p.) (Morani et al., 2013), Mesyl Sal B (0.3 and $1 \mathrm{mg} / \mathrm{kg}$, i.p.) (Simonson et al., 2015), and Ethoxymethyl ether Sal B (0.1 and $0.3 \mathrm{mg} / \mathrm{kg}$, i.p.) (Ewald et al., 2017) also attenuated cocaine-induced drug seeking in male Sprague-Dawley rats. However, the clinical utility of KOPr agonists is limited due to their dysphoric, aversive (Land et al., 2009), anxiogenic (Gillett et al., 2013), pro-depressive (Mague et al., 2003) and sedative effects (Gallantine and Meert, 2008) (see Table 4.1).

To the best of our knowledge, this is a first study to evaluate the effects of our novel dual KOPr/DOPr agonist for cocaine-induce drug seeking in male Sprague-Dawley rats. A strategy to avoid the KOPr-mediated side effects is to target multiple opioid receptors concurrently (Balboni et al., 2002; Dietis et al., 2009; Morphy et al., 2004; Morphy and Rankovic, 2009). We show that MP1104 (0.3 and $1 \mathrm{mg} / \mathrm{kg}$ ) attenuated drug seeking behaviour in rats (Atigari et al., 2019). Previously, a study in mice showed that MP1104 (1 mg/kg/s.c.) attenuated the rewarding effects of cocaine in CPP tests (Varadi et al., 2015). However, since the self-administration model is considered the gold standard for studying drug abuse preclinically (Carter and Griffiths, 2009), we studied the anti-cocaine effects of MP1104 using this model.

In this study, we found that rats that pre-treated with the KOPr antagonist, nor-BNI, significantly blocked the attenuation of drug seeking behaviour in rats (see Figure 3.2). This signifies that the 
reduced drug seeking behaviour shown by MP1104 in rats is via KOPr mediation. It is noteworthy that while KOPr and DOPr antagonists partially block the impact of MP1104 $(1 \mathrm{mg} / \mathrm{kg})$ on drug seeking behaviour in rats, the overall effect of MP1104 still seems well short of the vehicle treated rats (see Figure 3.2). This may be due to the sedative effects of MP1104 caused at this dose (1 $\mathrm{mg} / \mathrm{kg}$ ) (see Figure 3.10). It is possible that nor-BNI may not completely reverse the $\mathrm{KOPr}$ mediated sedative effects, only the KOPr mediated effects on cocaine-induced reinstatement. Due to its long-lasting actions ( $>8$ weeks), nor-BNI was administered in the last session (Endoh et al., 1992), which may contribute to the observed effects. In this study, we did not show the effects of NTI or nor-BNI alone on cocaine seeking and in the CPP models. However, previous literature has been explored to provide information in regards to this. Nor-BNI did not alter cocaine selfadministration behaviour in rats (10 mg/kg, s.c.) (Glick et al., 1995) or rhesus monkeys (3.2 $\mathrm{mg} / \mathrm{kg}$,i.v.) (Negus et al., 1997). At 3.2 or $10 \mathrm{mg} / \mathrm{kg}$, intramuscular (i.m.), nor-BNI had no effect on cocaine choice or extended-access cocaine intake (Hutsell et al., 2016; Negus, 2004) in rhesus monkeys, but decreased the intake of a threshold dose of cocaine ( $30 \mu \mathrm{g} /$ infusion) in drug naïve rats (Kuzmin et al., 1998). It also had no effect on cocaine intake when cocaine was offered at higher doses (60 $\mu \mathrm{g} /$ infusion), indicating that although nor-BNI may decrease sensitivity to cocaine, possibly because of its 'rewarding' effects through $\mathrm{KOPr}$ antagonism, it does not completely prevent cocaine intake (Kuzmin et al., 1998). Previously, a study showed that NTI (0.1 $-0.3 \mathrm{mg} / \mathrm{kg}$ ) failed to produce an effect in cocaine $(10 \mathrm{mg} / \mathrm{kg}) \mathrm{CPP}$ in male Sprague-Dawley rats and NTI by itself did not induce significant place conditioning (de Vries et al., 1995). In the same study NTI at $10 \mathrm{mg} / \mathrm{kg}$ but not lower doses $(0.03-3 \mathrm{mg} / \mathrm{kg})$ reduced the number of cocaine infusions in cocaine self-administering rats. This effect was seen over a wide range of cocaine doses 0.25 or $1 \mathrm{mg} / \mathrm{kg} /$ infusion. A study by Reid et al. (1995), also found that NTI (3 or $10 \mathrm{mg} / \mathrm{kg}$ ) decreased cocaine lever press responses in rat self-administration model regardless of the schedule of reinforcement. Also in rhesus monkeys, NTI administration (i.v.) produced decreases in cocaine self-administration. However, these effects were inconsistent across animals and sessions and were not dose-related (Negus et al., 1995). Furthermore, a recent study showed that intra-NAc infusions of NTI $(1 \mu \mathrm{g})$ in male Sprague-Dawley rats trained to self-administer cocaine $(0.75 \mathrm{mg} / \mathrm{kg}, 10$ days, $6 \mathrm{~h} /$ day), followed by either a 1-day or a 30-day period of forced abstinence and subsequent testing for cue-induced cocaine seeking behaviour (without cocaine reinforcement), showed no significant effect on cocaine seeking behaviour (Dikshtein et al., 2013). These studies suggest that endogenous levels of KOPr and DOPr activating peptides have limited effects on cocaine seeking behaviour, although this concept warrants 
full investigation. As one of the future directions, it would be worthwhile to test the effects of $\mathrm{NTI}$ or nor-BNI alone in cocaine primed reinstatement paradigm.

\subsubsection{MP1104 effect on cocaine taking behaviour}

To further explore the drug taking behaviour in rats, we used dose-response cocaine selfadministration tests. This model helps to assess the reinforcing strength of drugs of abuse in animals. It has been suggested that medications which only alter the effects of drugs of abuse will be less efficient, whereas those that shift the dose-response curve downward will have most general therapeutic utility (Mello and Negus, 1996).

In this study, we aimed to determine whether MP1104 attenuates the reinforcing effects of cocaine. We found that cocaine infusions between $0.15 \mathrm{mg} / \mathrm{kg}$ and $0.5 \mathrm{mg} / \mathrm{kg}$ showed high levels of responding (Figure 3.3). The standard dose of cocaine selected for performing cocaine seeking and taking behaviours in rats, in our laboratory has been $0.5 \mathrm{mg} / \mathrm{kg}$ (Ewald et al., 2017; Morani et al., 2009; Simonson et al., 2015). However, we wanted to re-affirm whether this dose of cocaine is sufficient to induce reinforcing effects using dose-response cocaine self-administration tests. In agreement with the previous studies, we found that animals that received vehicle showed elevated response between 0.15 and $0.5 \mathrm{mg} / \mathrm{kg}$ dose of cocaine.

Here, we show, that throughout the entire dose range of cocaine $(0.03,0.15,0.5,1$ and 2 $\mathrm{mg} / \mathrm{kg}$ /infusion), MP1104 dose-dependently caused a downward shift (Figure 3.3) in the cocaine dose-response curve suggesting that the reinforcing effects of cocaine are attenuated by MP1104 (Atigari et al., 2019). This is the first study to show the effects of a dual KOPr/DOPr agonist in a model that measures the cocaine taking behaviour in rats.

It has been suggested that every self-administering drug dose has two competing effects: reinforcing effects that increases the probability of another response and the rate altering effects that either increase or decrease ongoing behaviour. Studies have demonstrated that an inverse relationship exists between rate of responding and the magnitude of reinforcement in rats selfadministering cocaine (Pickens and Thompson, 1968), methamphetamine (Pickens et al., 1967) and d-methamphetamine (Pickens and Harris, 1968) and in rhesus monkeys self-administering nicotine (Deneau and Inoki, 1967) and cocaine (Wilson et al., 1971). However, in all the above 
mentioned studies, across a wide range of unit dosages, daily drug intake was independent of the magnitude of reinforcement. This suggests that some mechanism is involved in regulating or limiting drug intake. There is evidence that large dosages of the psychomotor stimulants such as cocaine produce aversive effects (Wilson and Schuster, 1972), and it is these aversive effects which limit the amount of psychomotor stimulants which are self-administered. It is important to note that unrestricted access to cocaine has been reported to cause overdose and death in animals. Johanson et al. (1976) showed that when rhesus monkeys self-administered damphetamine $(0.05 \mathrm{mg} / \mathrm{kg})$, d-methamphetamine $(0.025 \mathrm{mg} / \mathrm{kg})$, and cocaine $(0.2 \mathrm{mg} / \mathrm{kg})$ in an unlimited access pattern, they died. Also, unlimited access to cocaine ( 0.25 and $1 \mathrm{mg} / \mathrm{kg}$, i.v.), and a combination of cocaine $(1 \mathrm{mg} / \mathrm{kg})$ and codeine $(0.5$ and $2.5 \mathrm{mg} / \mathrm{kg})$, or morphine $(2.5 \mathrm{mg} / \mathrm{kg})$ (all drugs administered via i.v. infusions), caused convulsions and overdose in most of the monkeys (Macaca mulatta) within 2-6 weeks (Deneau et al., 1969). In this study, different cocaine doses were delivered in restricted manner and assessed for the cocaine taking behaviour in rats.

A drawback of this study is that it was not feasible to test the effects produced by administering specific antagonists for KOPr (nor-BNI, $10 \mathrm{mg} / \mathrm{kg}$, s.c.), DOPr (NTI, $15 \mathrm{mg} / \mathrm{kg}$, s.c.) and MOPr ( $\beta$ FNA, $10 \mathrm{mg} / \mathrm{kg}$, s.c.) alone, nor in combination with MP1104. This study allows us to confirm that MP1104 is effective in shifting the dose response curve downward, however it does not allow us to tease out the actions at KOPr, MOPr, and DOPr in mediating this effect. However, as a future direction of this thesis research we would like to measure the effects produced by the abovementioned specific antagonists. It is noteworthy that nor-BNI has long-lasting effects (Horan et al., 1992; Kishioka et al., 2013) and $\beta$-FNA is an irreversible MOPr antagonist (Katsuura and Taha, 2014; Qi et al., 1990). Hence, using a within-subject Latin square would not be ideal, and this will result in using more number of animals to test the effects. However, considering the length of time taken to produce the data, the risk of losing lines in self-administration test, and that a between subject design needs much more animals, we did not study the antagonists effects using this test. 


\subsubsection{Effect of MP1104 on dopamine transporter function}

Drugs of abuse act by increasing DA levels. Because KOPr agonists are known to increase DAT function and reduce DA levels we also chose to evaluate the effects of MP1104 on DAT function using RDEV techniques in rat dStr and NAc tissues. These tissues were selected as they are key brain regions involved in modulating reward behaviours.

Previous studies found DAT to be present in the VTA, dStr, SN and NAc (Boja and Kuhar, 1989; Ciliax et al., 1995). DAT was also found to co-localise with KOPr in the VTA, dStr, NAc, SN, amygdala, pFC. It was shown that KOPr and DAT are placed in close proximity in DA nerve terminals (Ciliax et al., 1999; Ciliax et al., 1995; Simonin et al., 1995), suggesting KOPr may play a role in regulating DAT and thereby DA levels (Svingos et al., 2001) (Figure 1.1).

\subsubsection{MP1104 effect on DAT function in dStr and NAc brain regions}

Acute treatment with MP1104 increased DAT function in isolated rat dStr and NAc using RDEV. MP1104 caused an increase in DA reuptake by DAT that was nor-BNI reversible in both the dStr and NAC, indicating that the effect is KOPr mediated (Figure 3.4C \&D) (also refer to Atigari et al., 2019). It is important to note that NTI addition had no effect on MP1104 induced increases in DAT function in both brain regions. This suggests that the decreased levels in DA measured is contributed by increased DAT function in these brain regions following KOPr activation. RDEV electrochemical techniques are useful as it provides information kinetic data with $V_{\max }$ and $K_{m}$ values, which further gives information on how DAT is being modulated by KOPr activation. Changes in $V_{\max }$ with no change in $\mathrm{K}_{\mathrm{m}}$ would indicate alterations in DAT cell surface expression, whereby an increase in $V_{\max }$ indicates either an increase in transporter activity, an increase in cell surface expression, or both. On the other hand, changes in $\mathrm{K}_{m}$ indicate a change in the affinity of a substrate for the transporter, with an increase in $\mathrm{K}_{\mathrm{m}}$ indicating a decrease in binding affinity and vice versa. MP1104 (1 nM and $500 \mathrm{nM}$ ) caused a significant increase in $\mathrm{V}_{\max }$ in dStr and NAc rat brain regions (Figure 3.4A \&B) (also refer to Atigari et al., 2019). An increase in Vmax is often associated with an increase in cell surface expression of transporters. However, changes in cell surface expression is measured by cell-surface biotinylation and total internal reflection microscopy (TIRFM) experiments. Simonson et al (2015) performed these experiments and showed that the KOPr agonists, Sal A $(10 \mu \mathrm{M})$ and U69,593 $(10 \mu \mathrm{M})$ increased DA uptake by DAT, producing a significant increase in DAT cell surface expression, however, the Sal A analogue, Mesyl 
Sal $B$, increased the $V_{\max }$ of DA uptake in the RDEV model without increasing DAT cell surface expression in KOPr and DAT expressing HEK-293 cells. This indicates a difference in regulation of DAT. Further evidence has shown that Sal $\mathrm{A}(10 \mu \mathrm{M})$ increased DAT cell surface expression in KOPr and DAT co-expressing EM4 cells, as well as striatal synaptosomes (Kivell et al., 2014a). It may be possible that the differential mechanisms involved in regulation of DAT will correlate to the KOPrmediated side effects observed. However, further studies involving KOPr signal transductions are required to determine if such correlation exists. Activation of KOPr also directly modulates DA release (Clow and Jhamandas, 1988; Xi et al., 1998), and the combination of factors that regulate both release and uptake in each specific brain region will contribute to levels of synaptic DA. Measuring both release and uptake of DA in specific brain regions is complex. Zimmer et al., (2000) adapted the 'indicator diffusion' method applied to the microdialysis technique which is capable of measuring DAT function, and other microdialysis protocols can measure DA release (Jaquins-Gerstl and Michael, 2015; Schultz, 2007), however these experiments require extensive expertise to perform. Future studies using in vivo voltammetry studies would give a better information on DAT function and DA release.

It is noteworthy that KOPr activation has also been shown to regulate SERT function (Schenk et al., 2000), and activation of SERT by U69,593 led to decreased serotonin uptake in rat striatal synaptosomes (Ramamoorthy et al. 2002). Also, studies have shown that DA uptake can also take place via the NET (Carboni et al., 1990), and SERT (Larsen et al., 2011). Further studies are needed to evaluate whether SERT and NET are involved in modulating DA levels following MP1104 administration. One way to measure DAT function in vitro is by using $\mathrm{ASP}^{+}$(4-(4-(dimethylamino)styryl)-N-methylpyridinium), which is a lipid-dependant fluorophore derived from chemical modification to the neurotoxin 1-methyl-4-phenylpyridinium (MPP+) (Magrassi et al., 1987). ASP+ shows substrate specificity for monoamine transporters, SERT (Oz et al., 2010), NET (Schwartz et al., 2003), and DAT (Bolan et al., 2007; Kivell et al., 2014a; Zapata et al., 2007) and will appear to fluoresce significantly only within cells expressing these transporters (example: DAT VS SERT or DAT Vs NET etc). SERT activity can also be measured using RDEV. Hagan et al. (2010) provided the first use of RDEV to measure SERT function in mouse whole brain synaptosomes. Simonson (2011), using RDEV showed a $25 \%$ decrease in uptake of $2 \mu \mathrm{M} 5-\mathrm{HT}$ occurred following U50,488H (500 nM), and Sal A (500 nM) administration to rat striatal tissue, demonstrating that KOPr also modulates SERT in the rat striatum. These studies provide means to study monoamine transporter functions via available techniques in ex vivo and in vitro models. 


\subsubsection{DA uptake by DAT in dStr and NAc brain regions}

Following administration of drugs of abuse (ethanol, opioids, amphetamine, nicotine and cocaine) microdialysis studies performed in freely moving rats showed DA levels were increased (Di Chiara and Imperato, 1988a). The dStr was chosen as a region of interest to investigate changes in DAT function following KOPr administration as increased DA overflow in the dStr is connected with the locomotor activation effects of psychostimulants, linking it to the ability to carry out motivated behaviour (Fink and Smith, 1979). The dStr has also been shown to play a role in the persistence of responding for a drug-associated stimulus, also the reacquiring to the drug-associated stimulus following extinction period (Di Ciano et al., 2008). The NAc has also been shown to be involved in the initiation of rewarding processes (Self et al., 1994), maintenance of drug taking behaviour (Carelli and ljames, 2000) and relapse to drug addiction (Self, 2004).

Both the dorsal and ventral striatal regions contain terminals of the nigro-striatal and mesolimbic DA neurons, and co-express high levels of KOPr and DAT (Boja and Kuhar, 1989; Gulley and Zahniser, 2003; Marshall et al., 1990; Meshul and McGinty, 2000; Richfield, 1991). In addition, it has previously been reported that DA uptake in the dStr and NAc is primarily dependent on DAT (Morón et al., 2002), and that in striatal axons and varicosities, electron microscopy revealed that KOPr is located in close vicinity to DAT (Svingos et al., 2001). Taken together, these studies indicate that the KOPr is strategically located in the striatum to modulate DA uptake via regulation of DAT. A study by Simonson et al. (2015), using the same RDEV methods utilised in this study, showed that brain regions have varying rates of DA uptake, with the striatum showing the largest uptake, followed by the NAc, then the medial pFC (Simonson et al., 2015). These results directly correlate with the amount of DAT that is expressed in each brain region, with the striatum having the highest DAT density, followed by the NAc and then the medial pFC (Freed et al., 1995; Sesack et al., 1998). Moreover, there is evidence of low DAT expression in the VTA, as DAT is localised to synaptic regions (Shimada et al., 1992). Hence, in this study we chose to evaluate dStr and NAc brain regions for DA uptake and DAT functions.

While previous studies have shown KOPr agonists attenuate DA release (Chefer et al., 2000; Zhang et al., 2004a) and increase DA uptake via DAT (Thompson et al., 2000), these studies utilised selective KOPr agonists. Therefore, we sought to determine whether the mixed actions of MP1104 were sufficient to modulate DAT function. We found that MP1104 increased DA uptake by increasing DAT function in both dStr and NAc rat brain regions (Figure 3.4 A, B, C \& D). 
It is likely that these effects are due to direct interactions of KOPr and DAT, as previous studies evaluating co-expression of KOPr and DAT found that KOPr and DAT form interacting complexes in striatal synaptosomes, and that this KOPr and DAT interaction is enhanced by administration of KOPr agonists (Kivell et al., 2014a). Several studies have also reported that administration of KOPr agonists and endogenous KOPr activation reduces DA release (Carlezon et al., 2006; Gray et al., 1999), and there are differences in the regulation of DAT in NAc and dStr brain regions (Thompson et al., 2000). Moreover, DA regulation between these regions also vary (Richards and Zahniser, 2009; Wu et al., 2001). 


\subsection{Anti-nociception studies}

To explore the preclinical development of non-addictive pain medications we chose to evaluate the nociceptive and chemotherapy-induced neuropathic pain using the warm-water tail withdrawal assay and the paclitaxel-induced neuropathic pain model respectively, in rodents.

\subsubsection{Effects of MP1104 in warm-water tail withdrawal assay}

In this study, we measured the onset and duration of action of MP1104, a mixed KOPr/DOPr and MOPr agonist, in the warm-water tail withdrawal assay in rats over time following a single i.p. injection. This assay is used to investigate anti-nociceptive effects following a thermal nociceptive stimulus, mediated via spinal cord reflexes. It is noteworthy that it important to evaluate whether MP1104 crosses the blood brain barrier, as this is a major limitation in developing CNS drugs.

MP1104 at both doses $(0.3$ and $0.6 \mathrm{mg} / \mathrm{kg}$ ), showed longer duration of action with effects lasting up to $8 \mathrm{~h}$ compared to morphine which is effective for up to $2 \mathrm{~h}$ (Figure $3.5 \mathrm{~A}$ ). The onset of action of MP1104 was found to be around $45 \mathrm{~min}$ at both doses, whereas morphine showed a significant anti-nociceptive effects from 30 min following i.p. administration. The increase in anti-nociceptive duration indicates that MP1104 shows improvements over the current clinically used mu-opioid drugs such as morphine used to treat severe acute pain.

To further evaluate the specific opioid receptor responsible for the anti-nociceptive effects, we used selective antagonists to block MOPr, DOPr or KOPr receptors. Upon blockade with the MOPr antagonist, $\beta$-FNA (10 mg/kg, s.c.), KOPr antagonist, nor-BNI (10 mg $/ \mathrm{kg}$, s.c.) or DOPr antagonist, $\mathrm{NTI}$ (15 mg/kg, s.c.), we found that the anti-nociceptive effects were mediated via KOPr and DOPr activity, as simultaneous blockade with nor-BNI and NTI completely blocked anti-nociceptive effects of MP1104 (0.6 mg/kg, i.p.). The results showed that MOPr had no role in inducing antipain effects since $\beta$-FNA had no significant effect (Figure 3.5A \& B).

While this may seem surprising, this data obtained from rats is consistent with the results observed in anti-nociceptive studies performed in mice using MP1104. For example, antinociception effects in male $\mathrm{CD} 1$ and $\mathrm{C} 57 \mathrm{BL} / 6 \mathrm{~J}$ mice were determined using a radiant heat $\left(55^{\circ} \mathrm{C}\right)$ tail-flick technique (Varadi et al., 2015). MP1104 (0.5 mg/kg, s.c), was insensitive to the muopioud antagonist $\beta$-FNA (40 mg/kg, s.c) and a NOPr antagonist (J-113,397; $30 \mathrm{mg} / \mathrm{kg}$, s.c.). However simultaneous administration of nor-BNI (10 mg/kg, s.c.) and NTI (20 mg/kg, s.c.) 
prevented anti-nociceptive responses. Similarly, in C57BL/6J mice and in MOPr KO mice, significant anti-nociception was observed following MP1104 administration, but the effects of MP1104 were significantly attenuated in KOPr KO mice and wild-type mice pre-treated with the DOPr antagonist NTI (20 mg/kg, s.c.), indicating the anti-nociceptive effects were KOPr and DOPr mediated in mice (Varadi et al., 2015).

The anti-nociceptive dose-response effects of MP1104 (via s.c. injections for slow release of the drug) were evaluated using the acute warm-water tail withdrawal assay. MP1104 was found to be potent in rats $\left(E_{50}=0.58 \mathrm{mg} / \mathrm{kg}\right)$ and mice $\left(\mathrm{ED}_{50}=0.35 \mathrm{mg} / \mathrm{kg}\right) . \mathrm{MP} 1104$ was found to have higher efficacy in mice $\left(E_{\max } \pm S E M=96.63 \pm 1.13\right)$ than in rats $\left(E_{\max } \pm S E M=51.31 \pm 1.877\right)$ (Figure 3.6A \& B; Table 3.1). One of the reason for such variation noticed is the possibility of species differences. A few studies have shown that the effects of KOPr vary in different species (DiMattio et al., 2015; Schattauer et al., 2017).

It is important to know that there is no one animal model that can evaluate the complex physiology of pain. Each model used has some validity for aspects of pain. For example, the warmwater tail withdrawal assay is a thermal nociceptive pain model, which uses thermal stimuli (warm-water) to measure the reflexive pain behaviour. This model involves spinal reflexes and does not require higher brain processes (Irwin et al., 1951), a drawback of the model. The use of other nociceptive thermal pain models like the hotplate test is helpful. The hotplate test involves supra-spinal reflex, the behaviours observed such as paw withdrawal and jumping to escape the thermal stimulus requires higher brain processing (Saadé and Jabbur, 1985; Casey et al., 1996). Another drawback is use of a hand-operated stopwatch. Instead, use of an automated apparatus to measure the latencies of tail withdrawal would have been preferred by the experimenter. Purchase of an automated apparatus for recording tail withdrawal latencies would be ideal. 


\subsubsection{Cumulative dose-response effects of MP1104 on mechanical and cold allodynia}

The dose-response effects were initially calculated to ascertain the efficacy and potency of each treatment. The effects of MP1104 were compared to morphine in order to make comparisons to a clinically available treatment. MP1104 was more potent than morphine in mechanical threshold testing and cold allodynia testing (Figure 3.7A \& B). This is the first study to show the effect of a mixed KOPr/DOPr agonist in reducing mechanical and cold allodynia in paclitaxel-induced neuropathic pain in male C57BL/6 mice strain. In the Kivell laboratory this model was recently established in mice, and there are significant species and strain differences observed in this model, hence effects of acute and chronic administration of MP1104 in CINP was assessed in mice.

Previously, a few studies reported the effects of MOPr agonists on paclitaxel-induced neuropathic pain in male Sprague-Dawley rats. The dose-response effects against paclitaxel-induced mechanical pain showed the order of potency of MOPr agonists as fentanyl > oxycodone > morphine (Mori et al., 2014). Methadone reduced both mechanical and thermal allodynia at doses $5 \mathrm{mg} / \mathrm{kg}$, i.p and $2.5 \mathrm{mg} / \mathrm{kg}$, i.p respectively, whereas morphine was effective at attenuating both mechanical and thermal allodynia at $2.5 \mathrm{mg} / \mathrm{kg}$, i.p dose (Pascual et al., 2010). The differences in strain have different effects. It has been reported that compared to other strains of mice C57BL/ 6 mice may be more resistant to the induction of mechanical allodynia by paclitaxel (Smith et al., 2004). It is noteworthy that although MOPr agonists like morphine are administered to cancer patients, morphine is not used in the treatment of CINP. The first line treatments for CINP are duloxetine (serotonin and norepinephrine reuptake inhibitor-SNRIs) and gabapentin (voltage gated $\mathrm{Ca}^{2+}$ channel modulating anticonvulsant). Clinical studies data show that duloxetine at an oral dose of $30 \mathrm{mg}$ daily for one week, followed by $60 \mathrm{mg}$ daily for four weeks reduced paclitaxel, oxaliplatin, docetaxel or cisplastin induced CINP compared to placebo in 231 patients (Smith et al., 2013). Although gabapentin demonstrated no difference to placebo in 115 patients with paclitaxel, docetaxel, cisplatin, vincristine, oxaliplatin, carboplatin or vinblastine CINP at an oral dose escalated up to $2700 \mathrm{mg}$ daily (Rao et al., 2007), preclinical studies found that gabapentin $(3-30 \mathrm{mg} / \mathrm{kg}$, i.p.) reduced paclitaxel-induced mechanical and thermal hyperalgesia (single treatment with paclitaxel at $4 \mathrm{mg} / \mathrm{kg}$, i.p.) in male ddY mice up to $24 \mathrm{~h}$ post treatment (Matsumoto et al., 2006) and in female BALB/c mice $(10-30 \mathrm{mg} / \mathrm{kg}$, i.p) reduced paclitaxel-induced thermal hyperalgesia $(2 \mathrm{mg} / \mathrm{kg}$, i.p., treatment with paclitaxel for five consecutive days) (Thangamani et al., 2013). Similarly, in male Sprague-Dawley rats, gabapentin $(100 \mathrm{mg} / \mathrm{kg}$, i.p.) reduced paclitaxel-induced mechanical allodynia $(2 \mathrm{mg} / \mathrm{kg}$, i.p., treatment with 
paclitaxel for four consecutive days) (Xiao et al., 2007). MOPr agonists are regarded as third-line treatment for CINP due to their addictive and adverse effects, including tolerance and respiratory depression (Chu et al., 2006; Finnerup et al., 2015). Due to these adverse effects associated with MOPr agonists, KOPr agonists could be developed as improved analgesics for treating CINP. KOPr agonists such as Sal A and $\beta$-tetrahydropyran Salvinorin B, an analogue of Sal A, and the prototypical KOPr agonist U50,488 have been shown to significantly supress mechanical and cold allodynia in paclitaxel-induced neuropathic pain in male B6-SJL mice (Paton et al., 2017) and in both male and female C57BL/6 mice (Paton, 2018). However, compared to pure KOPr agonists mixed opioid agonists have reduced side effects, hence MP1104 being a mixed opioid agonist is suggested to be a better candidate for clinical development.

In search of effective treatments to treat chronic pain, further effects of MP1104 were evaluated in a chronic pain model of CINP.

\subsubsection{Effect on chronic administration of MP1104 agonist on mechanical and cold allodynia}

The effects of chronic administration of MP1104 and morphine treatments were assessed in male C57BL/6 mice following induction of stable CINP. Mechanical allodynia scores that were performed on day 15 , allowed mice to be evenly distributed across treatments groups, to avoid bias (see Appendix G). Chronic administration of MP1104, morphine or vehicle were administrated daily from day 16 - 38. We show that mechanical and cold allodynia scores were significantly improved in mice treated with MP1104 and morphine (Figure 3.8A \& B).

When mechanical allodynia was measured 1 hour following MP1104 administration $(1.2 \mathrm{mg} / \mathrm{kg}$, i.p./daily) there was a significant increase in the mechanical force required to elicit paw withdrawal in mice. This indicates that MP1104-treated mice improved mechanical allodynia. MP1104 reduced mechanical threshold back to baseline control levels (non-diseased control mice) (Figure 3.8A).

MP1104 was more effective in CINP than morphine (Figure 3.8A). Morphine (10 mg/kg, i.p./daily) reduced paclitaxel effects for up to 30 days but, until day 30 of our model, where mice became 
tolerant to the anti-nociceptive effects. Whereas, MP1104-treated mice produced antinociceptive effects on all days of treatment (16-38) and showed no tolerance.

MP1104 was also effective in reducing cold allodynia in mice with well-established paclitaxelinduced neuropathic pain. MP1104 and morphine treatment groups had significant effects on all days 16-38 (Figure 3.8B). MP1104 and morphine reduced cold response time compared to vehicle/paclitaxel treated mice (Figure 3.8D). Results revealed that MP1104 (1.2 mg/kg, i.p., daily) reduced the cold allodynia effects back to vehicle/vehicle levels (Figure 3.8B).

Recent studies performed in our laboratory, using the same CINP model have shown that selective KOPr agonists such as U50,488 and an analogue of Sal A, 16-Ethynyl Sal A (4 mg/kg i.p.), reduce allodynia when administered chronically in both male and female C57BL/6 mice (Paton, 2018). However, U50,488 (4 mg/kg i.p.) was more effective, in reducing mechanical and cold allodynia to baseline (non-CINP levels) over 23 days of KOPr treatment, and did not show tolerance. However, U50,488 is not suitable for clinical development for treating neuropathic pain due to side effects that include aversion, depression, anxiety, and sedation (see Table 4.1).

\subsubsection{DOPr role in neuropathic pain}

The role of the DOPr in the development and expression of neuropathic pain has been studied previously (Mika et al., 2001; Ong et al., 2016). Nadal et al. (2006) utilised the sciatic nerve ligation model of neuropathic pain to evaluate the role of the DOPr in wild-type and DOPr KO mice. Neuropathic pain was observed following both mechanical and thermal stimuli. However, the development of mechanical and thermal allodynia, and thermal hyperalgesia was significantly enhanced in DOPr KO mice. These results reveal that DOPr modulates neuropathic pain and identified a new potential therapeutic use for DOPr agonists. Furthermore, pharmacological and genetic data highlight DOPr agonists as a promising alternative to MOPr analgesics in the treatment of chronic pain (Bie and Pan, 2007; Cahill et al., 2007; Kabli and Cahill, 2007; Zhang et al., 2006). Hence, it is not surprising to observe such effective improvement to mechanical and cold allodynia with MP1104, which is a mixed opioid agonist acting at both KOPr and DOPr, with both receptor activities being efficient in treating neuropathic pain. What our study does highlight is the effectiveness of MP1104 following repeated administration without tolerence. This suggests the development of MP1104 for the treatment of CINP. 


\subsubsection{Dual KOPr/DOPr effects in pain}

Few compounds possessing dual KOPr/DOPr activity have been evaluated in pain models. Details of published studies and the models utilised to evaluate pain are shown in Table 1.2. A study by Taylor et al. (2015) showed that pre-treatment with the DOPr agonist 4-(alpha-(4-Allyl-2,5dimethyl-1-piperazinyl)-3-methoxybenzyl)-N,N-diethylbenzamide (SNC80) (5 mg/kg, s.c.), blocked the KOPr-mediated anti-nociception of U50,488 (10 mg/kg, s.c.) in male C57BI/6J mice using warm-water tail withdrawal assay. The study hypothesised that KOPr-mediated antinociception might be a form of stress-induced analgesia which may be blocked by the anxiolytic effects of DOPr agonists. To test their hypothesis, stress-induced analgesia was generated via forced swimming, a paradigm previously shown to induce a KOPr dependent analgesic response (McLaughlin et al., 2003). Results showed that pre-treatment with SNC80 effectively blocked the stress-induced analgesia in this paradigm. The study also showed that the non-opioid anxiolytic agent, diazepam ( $1 \mathrm{mg} / \mathrm{kg}$, s.c.) blocked the anti-nociceptive effects of $U 50,488 \mathrm{H}$ in a manner similar to SNC80. These results suggest that systemically administered KOPr agonists produce anti-nociception through a stress-induced mechanism, which can be blocked by distinct classes of anxiolytics, including DOPr agonists and identifies DOPr agonists as potential agents for reversing stress-driven addictive and depressive behaviours mediated through KOPr activation. Most of the analgesic efficacy of systemically administered KOPr agonists is mediated through central targets, as KOPr are found throughout the CNS and the PNS (Minami et al., 1993). Systemic U50,488H produced a strong anti-nociceptive effect, compared to peripherally restricted KOPr agonists (Barber et al., 1994; Stein et al., 1989). While systemic administration of DOPr agonists has minimal effects on anti-nociception, they do possess strong anxiolytic and anti-depressant effects (Perrine et al., 2006; Saitoh et al., 2004).

Depending on the route of administration, different populations of receptors are targeted. In support of this, Miaskowski et al., (1990) found that in the spinal cord, $\operatorname{KOPr}(U 50,488)$ and DOPr agonists (DPDPE) injected intrathecally (i.t.) synergized to produce an elevated anti-nociceptive response (Miaskowski et al., 1990). Another study found a small molecule agonist of DOPr/KOPr heterodimers, 6'-guanidinonaltrindole (6'-GNTI), selectively activated DOPr/KOPr heterodimers but not KOPr or DOPr homomers and showed anti-nociceptive effects in the mouse tailwithdrawal assay when administered in the spinal cord but not in the brain (Waldhoer et al., 2005). I.t. injections of $6^{\prime}$-GNTI produced anti-nociceptive effects with an ED $_{50}$ of $0.45 \mathrm{nmol}$ (per mouse) which was fully blocked by nor-BNI and NTI, further a KOPr/DOPr selective bivalent 
antagonist KDN-21 (Bhushan et al., 2004) also completely blocked 6'-GNTI mediated analgesia. However, 6'-GNTI produced little to no analgesia when it was administered intracerebroventricularly (i.c.v). Together, the data suggests that opioid receptor heterodimers are indeed a distinct functional signalling unit and could provide a target for development of tissue-selective analgesics with reduced side effects. Many of the side effects associated with the use of morphine are greatly reduced or eliminated when the drug is administered directly into the spinal cord (Fairbanks, 2003). Thus, this study proposes that drugs that selectively target heterodimers unique to the spinal cord could potentially produce fewer side effects. In this study, only s.c. (cumulative dose-response effects of MP1104 in CINP model and the warm-water tail withdrawal assay in mice) and i.p. (measuring time course effects of MP1104 in warm-water tail withdrawal assay in rats and chronic MP1104 administration in CINP model in mice) injections were performed in pain assays. It will be interesting to observe the effects of MP1104 via i.t. or i.c.v administration in rats and mice for anti-nociceptive effects and compare the results with the previous studies performed using mixed KOPr/DOPr agonists.

\subsubsection{Limitations with CINP model}

It is important to note that there are a few limitations with the CINP model used in this study. This study uses simplified up-down system, where five applications for every measurement which removes the variation in the amount of stimulation each animal receives (Bonin et al., 2014). Instead, the use of electronic von Frey equipment would eliminate the multiple application and the resultant recordings measured in force calculated by estimating the threshold based on which the filament causes the paw withdrawal. The acetone model of cold allodynia has a problem that acetone application may have influence on the skin or the olfactory cues, which may interfere with the interpretation of the results. Another drawback of the CINP model is that the effects of MP1104 was compared to morphine with the goal to find improved opioid receptor agonists with no addictive or tolerance-producing effects. One benefit of using MOPr agonists to treat CINP is immediate pain relief compared to the first line treatments such as gabapentin or duloxetine Morphine is not effective overtime, and repeated use of morphine produces tolerance (Chu et al., 2006), and other conditions like itch (Katcher and Walsh, 1999). Therefore, as a future direction of the study, the effects of MP1104 could be compared to gabapentin or duloxetine, which are 
clinically available first line treatments for CINP. Finally, it would be interesting to understand the effects of MP1104 on CINP by blocking the effects of MP1104 in mice by selective antagonists for $\operatorname{MOPr}(\beta-F N A), K O P r$ (nor-BNI), or DOPr (NTI) or use specific opioid receptor KO mice. The MOPr $\mathrm{KO}$ or KOPr KO or DOPr KO mice could be administered with paclitaxel and test the changes in induction of mechanical and cold allodynia. 


\subsection{Side effects}

Side effects limit the clinical development of currently available KOPr agonists (Mello and Negus, 2000; Walsh et al., 2001). KOPr activation is known to cause sedation (Mello and Negus, 2000), aversion (Chefer et al., 2013; Sufka et al., 2014; Suzuki et al., 1992b), anxiety (Valdez and Harshberger, 2012) and depression (Carlezon et al., 2006; Mague et al., 2003) in animals (see Table 4.1 below). Similar effects are also observed in humans with side effects including psychomimetic effects (Pfeiffer et al., 1986), sedation (Walsh et al., 2001), insomnia, and dysphoria (Glick et al., 1998). DOPr agonists have both anxiolytic and anti-depressant effects (Filliol et al., 2000; Torregrossa et al., 2006) and are also known to produce seizures (Bilsky et al., 1995; Comer et al., 1993; Jutkiewicz et al., 2006; Lutz and Kieffer, 2013a) which severely limits their clinical use. 


\section{Table 4.1: Acute effects of KOPr agonists on KOPr-mediated adverse effects}

\begin{tabular}{|c|c|c|c|c|}
\hline \multirow{2}{*}{$\begin{array}{l}\text { Behavioural tests to } \\
\text { assess side effects }\end{array}$} & \multicolumn{4}{|c|}{ KOPr agonist } \\
\hline & U50,488 & U69,593 & Salvinorin A & Animals \\
\hline \multirow[t]{2}{*}{$\begin{array}{l}\text { Anxiety } \\
\text { (Elevated plus maze } \\
\text { test) }\end{array}$} & $\begin{array}{c}\uparrow^{(1,2)} \\
5,10, \text { i.p. }\end{array}$ & - & $\begin{array}{c}\downarrow^{(3)} \\
0.1-160 \mu g / k g, \text { s.c. } \\
\uparrow \\
0.3,1.0, \text { i.p. }\end{array}$ & $\begin{array}{l}\text { Male Wistar rats } \\
(1,2) \\
\text { Male Sprague- } \\
\text { Dawley rats }{ }^{(3)}\end{array}$ \\
\hline & $\begin{array}{l}\text { 个 }(4,5) \\
5, \text { i.p. }\end{array}$ & - & - & $\begin{array}{l}\text { Male } \mathrm{C} 57 \mathrm{BL} / 6 \\
\text { mice }\end{array}$ \\
\hline $\begin{array}{l}\text { Anxiety } \\
\text { (Light and dark test) }\end{array}$ & - & - & $\begin{array}{c}\text { 个(6) } \\
1.0, \text { i.p. }\end{array}$ & $\begin{array}{l}\text { Male Sprague- } \\
\text { Dawley rats }\end{array}$ \\
\hline \multirow[t]{2}{*}{$\begin{array}{l}\text { Conditioned place } \\
\text { aversion }\end{array}$} & $\begin{array}{l}\text { 个 }(7,8) \\
1, \text { s.c. } \\
\text { 个 (9) } \\
10 \text {, i.p. }\end{array}$ & $\begin{array}{c}\uparrow(10) \\
0.32, \text { s.c. }\end{array}$ & $\begin{array}{c}\uparrow^{(6,11)} \\
0.3-1.0, \text { i.p. }\end{array}$ & $\begin{array}{l}\text { Male Long Evans } \\
\text { rats }{ }^{(7)} \\
\text { Male Sprague- } \\
\text { Dawley } \\
\text { rats }^{(6,8,9,10,11)}\end{array}$ \\
\hline & $\begin{array}{l}\text { 个 (12) } \\
2, \text { s.c. }\end{array}$ & $\begin{array}{c}\uparrow^{(13)} \\
0.32, \text { s.c. }\end{array}$ & $\begin{array}{c}\text { 个(14) } \\
1.0-3.2, \text { i.p. }\end{array}$ & $\begin{array}{l}\text { Male C57BL/6 } \\
\text { mice }\end{array}$ \\
\hline \multirow[t]{2}{*}{$\begin{array}{l}\text { Depression (Intracranial } \\
\text { self-stimulation) }\end{array}$} & - & $\begin{array}{c}\uparrow^{(15-18)} \\
0.18-0.56, \text { i.p. }\end{array}$ & $\begin{array}{c}\text { 个 and } \downarrow{ }^{(19-22)} \\
1.0-4.0 \text {, i.p. } \\
\text { n.e. }{ }^{(19)} \\
0.25 \text {, i.p. }\end{array}$ & $\begin{array}{l}\text { Male Sprague- } \\
\text { Dawley rats }\end{array}$ \\
\hline & $\begin{array}{c}\uparrow^{(23)} \\
3.0,5.5, \text { i.p. }\end{array}$ & - & - & $\begin{array}{l}\text { Male C57BL/6 } \\
\text { mice }\end{array}$ \\
\hline
\end{tabular}




\begin{tabular}{|c|c|c|c|c|}
\hline \multirow{2}{*}{$\begin{array}{l}\text { Behavioural tests to } \\
\text { assess side effects }\end{array}$} & \multicolumn{4}{|c|}{ KOPr agonist } \\
\hline & U50,488 & U69,593 & Salvinorin A & Animals \\
\hline \multirow[t]{2}{*}{$\begin{array}{l}\text { Depression (Forced } \\
\text { Swim Test) }\end{array}$} & - & $\begin{array}{c}\uparrow^{(24)} \\
0.3-10, \text { i.p. }\end{array}$ & $\begin{array}{c}\uparrow^{(25)} \\
0.25-2.0, \text { i.p. } \\
\downarrow^{(3)} \\
10-1000 \mu \mathrm{g} / \mathrm{kg}, \text { s.c. }\end{array}$ & $\begin{array}{l}\text { Male Sprague- } \\
\text { Dawley rats }\end{array}$ \\
\hline & $\begin{array}{c}\uparrow^{(26)} \\
2.35, \text { s.c. }\end{array}$ & - & - & $\begin{array}{l}\text { Male Kunming } \\
\text { strain mice }\end{array}$ \\
\hline $\begin{array}{l}\text { Depression } \\
\text { (Tail suspension test) }\end{array}$ & - & - & $\begin{array}{c}\downarrow^{(3)} \\
0.001-1.0 \mu \mathrm{g} / \mathrm{kg}, \text { s.c. }\end{array}$ & Male Swiss mice \\
\hline $\begin{array}{l}\text { Depression } \\
\text { (Chronic mild stress) }\end{array}$ & - & - & $\begin{array}{c}\downarrow^{(27)} \\
1.0, \text { i.p. }\end{array}$ & $\begin{array}{l}\text { Male \& female } \\
\text { Long Evans rats }\end{array}$ \\
\hline \multirow[t]{2}{*}{$\begin{array}{l}\text { Sedation } \\
\text { (Locomotor } \\
\text { activity/Rotarod) }\end{array}$} & $\begin{array}{c}\uparrow^{(28)} \\
2.5-40, \text { s.c. }\end{array}$ & - & $\begin{array}{c}\text { n.e. }{ }^{(20,25)} \\
0.125-2.0 \text {, i.p. }\end{array}$ & $\begin{array}{l}\text { Male Sprague- } \\
\text { Dawley rats }\end{array}$ \\
\hline & $\begin{array}{c}\uparrow^{(29)} \\
3.32, \text { s.c. }\end{array}$ & - & - & $\begin{array}{l}\text { Male Kunming } \\
\text { strain mice }\end{array}$ \\
\hline $\begin{array}{l}\text { Sedation } \\
\text { (Unresponsive to } \\
\text { stimuli and postural } \\
\text { relaxation) }\end{array}$ & $\begin{array}{c}\uparrow^{(30)} \\
0.10 \text { per h, i.v. }\end{array}$ & $\begin{array}{l}\uparrow^{(31,32)} \\
0.01-0.056, \text { i.v. } \\
0.01-0.032, \text { s.c. }\end{array}$ & $\begin{array}{c}\uparrow^{(31)} \\
0.032-0.1 \text {, i.v. }\end{array}$ & $\begin{array}{l}\text { Male and female } \\
\text { rhesus monkeys }\end{array}$ \\
\hline $\begin{array}{l}\text { Sedation } \\
\text { (Inverted screen } \\
\text { performance / } \\
\text { Locomotor activity) }\end{array}$ & $\begin{array}{c}\uparrow^{(33)} \\
1.0,10, \text { i.p. }\end{array}$ & $\begin{array}{l}\uparrow^{(34)} \\
1.0, \text { i.p. }\end{array}$ & $\begin{array}{c}\uparrow^{(34)} \\
0.5-2.0, \text { i.p. } \\
\text { n.e. }{ }^{(3)} \\
0.001-1 \mu g / k g, \text { s.c. }\end{array}$ & $\begin{array}{l}\text { Male C57BL/6 } \\
\text { mice }^{(33)} \\
\text { Male Swiss } \\
\text { mice }^{(3,34)}\end{array}$ \\
\hline
\end{tabular}

$\downarrow=$ attenuation, $\uparrow=$ presence of effect/potentiation, - = study not found. Unless otherwise stated, doses are given in $\mathrm{mg} / \mathrm{kg}$. 
1) Gillett et al. (2013)

2) Valdez and Harshberger (2012)

3) Braida et al. (2009)

4) Bruchas et al. (2009)

5) Smith et al. (2012)

6) Ewald et al. (2017)

7) Leri and Franklin (2000)

8) Göktalay et al. (2006)

9) Culverhouse (2016)
10) Tejeda et al. (2013)

11) Sufka et al. (2014)

12) Skoubis et al. (2001)

13) Chefer et al. (2013)

14) Zhang et al. (2005)

15) Bonano et al. (2014)

16) Tomasiewicz et al. (2008)

17) Do Carmo et al. (2009)

18) Todtenkopf et al. (2004)
19) Ebner et al. (2010)

20) Carlezon et al. (2006)

21) Potter et al. (2011)

22) Béguin et al. (2009)

23) DiNieri et al. (2009)

24) Mague et al. (2003)

25) Morani et al. (2009)

26) Zhang et al. (2015)

27) Harden et al. (2012)
28) Gallantine and Meert (2008)

29) Wang et al. (2009)

30) Negus et al. (1997)

31) Butelman et al. (2009)

32) Butelman and Kreek (2001)

33) Paris et al. (2011)

34) Fantegrossi et al. (2005) 
In this study, we evaluated MP1104 for the behavioural side effects of sedation, aversion, anxiety and depression. It is to be noted that MP1104 produced no seizures at doses 30 times its antinociceptive $E D_{50}$ value in mice (Varadi et al., 2015)

\subsubsection{Effect of MP1104 on sedation}

The sedative effects of MP1104 were investigated using the open field spontaneous locomotor activity tests. We evaluated the sedative effects in order to affirm whether the attenuation of responses in cocaine-primed self-administration testing was due to sedative effects of MP1104.

Traditional KOPr agonists like U50,488 have been shown to decrease spontaneous locomotor activity in rodents (Brust et al., 2016). Clinical studies have revealed that KOPr agonists also cause sedation (Knoll and Carlezon Jr, 2010). In this study, a low dose of MP1104 (0.3 mg/kg) showed no significant sedative effects, whereas MP1104 at $0.6 \mathrm{mg} / \mathrm{kg}$ showed a strong trend towards sedation and the higher $1 \mathrm{mg} / \mathrm{kg}$ dose caused significant sedative effects in rats (Figure 3.10E \& F) (also refer to Atigari et al., 2019). This is consistent with previous reports on selective KOPr agonists. For example Sal $A$ has been shown to have sedative effects in rats at $2 \mathrm{mg} / \mathrm{kg} / \mathrm{i} . \mathrm{p}$. (Chartoff et al., 2008) and in rhesus monkeys at $0.1 \mathrm{mg} / \mathrm{kg}$ dose (delivered via i.v infusion) (Butelman et al., 2009). However, Sal A $(0.3 \mathrm{mg} / \mathrm{kg}$, i.p.) at a lower dose that also reduced druginduced reinstatement did not alter spontaneous locomotion in rats (Morani and al, 2012). The habituated animals in this assay have very low locomotor activity; hence, further reduction is hard to measure due to a floor effect. A way to overcome this drawback is to use non-habituated animals for effective results, which is a future study.

It is noteworthy that the total distance travelled in CPA (Figure 3.11B) and the total arm entries in EPM (Figure 3.12B) were not attenuated by MP1104 (0.3 and $0.6 \mathrm{mg} / \mathrm{kg})$, additional supporting methods to evaluate motor behaviours. We conclude that MP1104 at the $0.3 \mathrm{mg} / \mathrm{kg}$ dose, which attenuated drug seeking behaviour in rats, was not due to sedation or the inability of rats to perform the operant task. However, at higher doses the reduced drug seeking behaviour may be due to the sedative effects exerted by MP1104. 


\subsubsection{Effect of MP1104 on aversion}

Aversive effects of MP1104 were investigated using a biased, three-chamber CPA model. U50,488 treated rats showed significant reduction in time spent in the U50,488-paired chamber signifying aversive properties (Figure 3.11A). These results support the previous findings of known aversive effects showed by U50, 488 in rats (10 mg/kg, i.p.) (Ewald et al., 2017; Suzuki et al., 1992b). Rats that were treated with MP1104 showed no change in time spent in the MP1104-paired chamber, indicating it has no aversive properties at $0.6 \mathrm{mg} / \mathrm{kg}$ (Atigari et al., 2019). Following antagonism of DOPr by the selective DOPr antagonist NTI (15 mg/kg, s.c), MP1104 showed significant aversivelike effects, with reduced time spent in the MP1104-paired chamber (Atigari et al., 2019). This gives strong support for the development of dual KOPr/DOPr agonists as both non-addictive analgesics and anti-addiction therapies to reduce the side effects seen with pure KOPr agonists.

To the best of our knowledge, this is the first study to demonstrate that DOPr agonist properties of MP1104 are able to negate the aversive effects produced by KOPr agonism.

\subsubsection{Effect of MP1104 on anxiety}

KOPr activation has been shown to have anxiogenic effects in mice (Smith et al., 2012), rats (Bruchas et al., 2009) and humans (Pfeiffer et al., 1986). These effects are antagonised by blocking KOPr with the antagonist nor-BNI (10 mg/kg, i.p.) and 5'-GNTI ( $3 \mathrm{nmol}$ in $3 \mu \mathrm{L}$, intracisternally) . Effects are also absent in prodynorphin KO mice (Wittmann et al., 2009), indicating a role of KOPr in mediating anxiety.

This study examined the anxiogenic effects of MP1104 using EPM test. An acute administration of MP1104 at 0.3 and $0.6 \mathrm{mg} / \mathrm{kg}$ showed no effect in time spent on open arm and total arm entries by rats (Figure 3.12A \&B) (also refer to Atigari et al., 2019). MP1104 (0.3 \& $0.6 \mathrm{mg} / \mathrm{kg}$ ) exhibited a non-significant trend towards increase in time spent on the open arm compared to vehicle controls.

Previously, U50,488 (5 and $10 \mathrm{mg} / \mathrm{kg}$, i.p.) was shown to produce significant anxiogenic effects by spending less time on the open arm of an EPM in rats (Valdez and Harshberger, 2012). However, previous work done by a student in our laboratory showed U50,488 (10 mg/kg, i.p.) did not produce significant anxiety-like effects but a $30 \%$ reduction of time spent on the open arm was observed by rats compared to vehicle controls (Culverhouse, 2016), suggesting a trend toward 
anxiogenic properties. Hence, due to cost effectiveness we chose to use a known anxiogenic drug, yohimbine as a positive control in this study. Results show that yohimbine, an $\alpha_{2}$ adrenoceptor antagonist showed a significant reduction in time spent in the open arm and the total arm entries in the EPM compared to vehicle controls (Figure 3.12A \&B), effects consistent with previous reports on yohimbine in animals (Pellow et al., 1985). MP1104 produced no anxiogenic effects at the dose that was effective in attenuating cocaine seeking $(0.3 \mathrm{mg} / \mathrm{kg})$.

\subsubsection{Effect of MP1104 on pro-depression}

Originally the establishment of FST was to measure anti-depressant effects of new compounds (Porsolt et al., 1977); however, pro-depressive effects are also observed utilising this model (Carlezon et al., 2006;). The time an animal spends immobile in the water-filled chamber is used as an approximation of a depressive state and the changes of this behaviour in comparison to vehicle control is used to assess the pro-depressive or anti-depressive effects of the new compounds.

In this experiment we showed that MP1104 $(0.3$ and $0.6 \mathrm{mg} / \mathrm{kg})$ did not significantly increase or decrease immobility, swimming or climbing activity times compared to vehicle controls (Figure 3.13A) (also refer to Atigari et al., 2019). This indicates that MP1104 produced no pro-depressive effects, unlike previous reports utilising pure KOPr agonists such as Sal A and a structural analogue methoxymethyl ether Sal B (Carlezon et al., 2006; Kivell et al., 2018). Both MP1104 doses (0.3 and $0.6 \mathrm{mg} / \mathrm{kg}$ ) showed no changes in mobility or immobility properties compared to vehicle control (Figure 3.13B).

Several studies have argued that the FST lacks face validity for depression. Behaviour in the FST is a reaction to the acute stressful stimulus, whereas human depression reflects a chronic subjective emotional state rather than a reaction to an individual stimulus (Commons et al., 2017; Molendijk et al., 2015). However, it is clear that the FST gives insight into the neural networks that coordinate the behavioural response to an acute inescapable challenge, and this behaviour in rodents is linked to changes in swim behaviour when exposed to KOPr agonists, therefore evaluation of KOPr agonists effects in the FST model can reflect changes in swim patterns during stress. This may be impaired in depression, but the rodent's behavioural response to a forced swim stressor does not directly reflect depression (De Kloet et al., 2016). In this study MP1104 had no effect on swim 
behaviours, therefore, unlike pure KOPr agonists that decrease swimming behaviours (Morani et al., 2012), MP1104 does not induce the same side effects. This is likely due to actions at DOPr and MOPr. 
Table 4.2: Summary of anti-cocaine, analgesic, and side effects produced by MP1104 in rats and mice

\begin{tabular}{|c|c|c|}
\hline MP1104 & Effects in rats & Effects in mice \\
\hline \multicolumn{3}{|l|}{ Anti-cocaine effects } \\
\hline Cocaine-prime reinstatement & $\downarrow^{*}(0.3 \& 1, \text { i.p. })^{(1)}$ & n.d. \\
\hline Cocaine self-administration & $\downarrow^{*}(0.3 \& 0.6, \text { i.p. })^{(1)}$ & n.d. \\
\hline Cocaine place preference & n.d. & $\downarrow(1, \text { s.c. })^{(2)}$ \\
\hline \multicolumn{3}{|l|}{$\begin{array}{l}\text { Modulation of DAT function } \\
\text { (isolated brain tissue) }\end{array}$} \\
\hline Dorsal striatum & $\uparrow^{*}(1 \mathrm{nM} \& 500 \mathrm{nM})^{(1)}$ & n.d. \\
\hline Nucleus accumbens & $\uparrow^{*}(1 \mathrm{nM} \& 500 \mathrm{nM})^{(1)}$ & n.d. \\
\hline \multicolumn{3}{|l|}{ Anti-nociceptive effects } \\
\hline \multicolumn{3}{|l|}{ Tail withdrawal assay } \\
\hline Cumulative dose-response effects & 个* $\left(E D_{50}=0.58\right.$, s.c. $)$ & $\begin{aligned} \text { 个 }\left(E D_{50}=\right. & 0.33, \text { s.c.; } \\
& C D 1 \text { mice })^{(2)} \\
\uparrow^{*}\left(E D_{50}=\right. & 0.35, \text { s.c. })\end{aligned}$ \\
\hline Anti-nociceptive effects & $\uparrow^{*}(0.3 \& 0.6$, s.c. $)$ & 个* $(0.5$, s.c. $)$ \\
\hline \multicolumn{3}{|l|}{ Paclitaxel-induced neuropathic pain } \\
\hline Acute dose-response effects & n.d. & 个* $\left(E_{50}=0.48\right.$, s.c. $)$ \\
\hline Chronic administration effects & n.d. & 个* $\left(E D_{80}=1.2\right.$, i.p. $)$ \\
\hline \multicolumn{3}{|l|}{ Side effects } \\
\hline Aversion (CPA) & n.e. $(0.3,0.6 \text {, i.p. })^{(1)}$ & n.e. $\left(1\right.$, s.c.) ${ }^{(2)}$ \\
\hline Anxiety (EPM) & n.e. $(0.3,0.6 \text {, i.p. })^{(1)}$ & n.d. \\
\hline Pro-depression (FST) & n.e. $(0.3,0.6 \text {, i.p. })^{(1)}$ & n.d. \\
\hline Locomotor activity & $\begin{array}{l}\text { n.e. }(0.3,0.6, \text { i.p. }) \\
\downarrow(1, \text { i.p. })(1)\end{array}$ & n.d. \\
\hline
\end{tabular}

$\uparrow=$ presence of effect/potentiation; $\downarrow=$ attenuation; n.e. $=$ no effect; n.d.= not determined; DAT = dopamine transporter; $\mathrm{CPA}=$ conditioned place aversion; $\mathrm{EPM}=$ elevated plus maze; $\mathrm{FST}=$ forced swim test. Unless otherwise stated, doses are given in $\mathrm{mg} / \mathrm{kg}$ and unless otherwise stated, male (Sprague-Dawley) rats and male C57BL/6 mice are used.

$\begin{array}{ll}\text { 1) (Atigari et al., 2019) } & \text { 2) (Varadi et al., 2015) }\end{array}$ 


\subsection{Limitations and future directions}

The ultimate intention of our preclinical research is to translate experimental findings into the clinic. There are currently no FDA approved therapeutics to treat addiction to psychostimulants and addiction to prescription opioids is growing (Cicero et al., 2014; Wenghofer et al., 2011). Due to their low abuse potential KOPr agonists have been suggested as potential anti-addiction agents (Kivell et al., 2014b). Acute administration of KOPr agonists have shown to reduce the rewarding properties of drugs of abuse in animals (refer to Table 1.1). With the opioid crisis on rise, studies are being encouraged on creating non-addictive analgesic therapeutics. KOPr agonists have also been shown to reduce the reinforcing effects of MOPr agonists when the two are selfadministered as mixtures in monkeys (Freeman et al., 2014; Naylor et al., 2015). However, adverse effects limits the clinical utility of pure KOPr agonists (refer to Table 4.1). Mixed opioid receptor agonists have been hypothesised to have better therapeutic profile with reduced side effects compared to selective opioid receptor agonists (Anand and Montgomery, 2018; Ananthan, 2006; Balboni et al., 2002; Bidlack, 2014; Greedy et al., 2013; Majumdar and Devi, 2018; Morphy and Rankovic, 2009; Váradi et al., 2016). Hence, this study evaluated the therapeutic potential of a mixed opioid agonist. Few of the limitations and future directions of this study have been discussed below.

The present study has shown that it is possible for a novel mixed KOPr/DOPr opioid receptor agonist to produce fewer negative side effects than selective KOPr agonists. MP1104, a mixed KOPr/DOPr agonist did no produce sedative, aversive, anxiogenic or pro-depressive effects in rats at low doses. However, at higher doses, MP1104, was found to produce sedative effects in rats. This effect can be further validated by using another preclinical model that assesses motor coordination and sedative effects of compounds, like the rotarod performance (Dunham and Miya, 1957) in mice and rats. In rotarod performance assays, rodents are trained to run on continuously rotating wheel and the sedative effects of novel compounds are tested by observing the altered performance of animals on the wheel. Testing of MP1104 treated rats on rotarod performance would have been useful in confirming whether MP1104 has sedative effects at higher doses and show the motor coordination side effects. A combination of behavioural models is always useful to supplement observations resulting from a single assay. However, in this study a single behavioural preclinical model has been performed for assessing a specific side effect due to time limitations. Nevertheless, a large number of studies have shown that the preclinical side effects models used in this study such as spontaneous locomotor activity test, CPA, EPM, FST to 
measure sedative, aversive, anxiogenic and pro-depressive effects respectively are widely accepted and have been validated for screening side effects (Carlezon et al., 2006; Chefer et al., 2013; Göktalay et al., 2006; Morani et al., 2013; Morani et al., 2012; Pliakas et al., 2001; Sufka et al., 2014).

Evaluating the effects of MP1104 on modulation of natural reward at their anti-cocaine doses is important. This is measured by sucrose self-administration model, where animals are required to press a lever in order to receive a sucrose pellet as a reinforcer. KOPr system plays a role in modulation of appetite on natural rewards such as sucrose (Lynch and Burns, 1990; Sipols et al., 2002). However, due to lack of time the effects of MP1104 on natural reward could not be tested. The effects of MP1104 are limited by confinement to a single mammalian model. Selfadministration models using non-human primates would be useful to test the ability of MP1104 to alter drug taking and drug seeking behaviours. The drug taking behaviour in rats, in this study, was assessed using a dose-response cocaine self-administration model. The major limitation of this model is the interference by the experimenter during each T.O period. To overcome this, the model can be programmed in a way that the cocaine infusions at different concentrations are delivered without the experimenter changing the cocaine syringes for each dose. This can be done by programming the delivery of cocaine at their respective concentrations by adjusting the volume of cocaine infusions delivered. However, this would not have been ideal as the volume of cocaine infusions delivered would not be consistent and would complicate the experimental procedure. To widen our understanding of the mixed opioid receptor agonist MP1104, its effect following chronic administration will need to be taken into consideration. Chronic KOPr activation with traditional KOPr agonists reported to exhibit drug seeking and addiction-like behaviours in monkeys (Negus, 2004). It would be interesting to see the results with chronic MP1104 administration in such models.

In this study the underlying mechanism for MP1104 producing anti-cocaine effects were investigated by studying its effect on DAT function. However, a possible shortcoming of this study would be testing the effects in brain regions from animals that had been treated in vivo with MP1104 rather than isolating and then treating with MP1104 agonist. Implementation of another mechanism to measure DAT function such as ASP+ uptake is another way of confirming the effects. Also, the use of use of HEK-293 cells, along with cells of neural lineage like neuro-2A cells (N2A) or SH-SY5Y could be used to confirm the regulatory effects of KOPr on DAT function in ASP ${ }^{+}$ and RDEV assays. In this context, future studies could also be conducted using both these assays, 
wherein DAT function and expression of cells treated with cocaine can be compared to cells pretreated with MP1104 prior to administration of cocaine. The results obtained from this study would provide information on cocaine-induced changes in DA uptake and explains a detailed mechanism of the anti-cocaine effects of MP1104. However, measuring these effects via this model is beyond the scope of this study as this is a time-consuming assay. Since, MP1104 being a mixed agonist the choice of positive control was a problem. However, use of U50,488 as positive control (as MP1104 is more KOPr oriented) is the future directions for this study.

The testing of cellular mechanisms to explain the behavioural effects is efficient. The cellular manifestations of an in vivo behaviour are very advantageous and a better way to fully understand the complexity of the KOPr and/or DOPr system. Recently, regional specific effects (using optogenetic models) have been identified that show KOPr activation in the ventral NAc shell elicits aversive-like behaviour and the dorsal region of the NAc shell inducing place preference (AlHasani et al., 2015). Therefore, finding ways of targeting these distinct KOPr expressing neuronal populations may be the key to develop KOPr agonists with reduced side effects.

The effects of MP1104, administered prior to cocaine, on cocaine-induced hyperactivity are yet to be evaluated. Previously it was reported that KOPr agonists inhibit cocaine-induced hyperactivity (Chartoff et al., 2008; Chefer et al., 2000). Since KOPr agonists have been reported to produce tolerance upon repeated administration (Suzuki et al., 2004), it would be interesting to observe the extent at which our novel mixed agonist can be given before showing tolerance effects. It is important to note here that MP1104 when given repeatedly in the CINP model did not produce tolerance.

KOPr agonist co-infusion with the opioid remifentanil or oxycodone have been shown to reduce the reinforcing and rewarding properties of the drugs in self-administration studies using rhesus monkeys (Freeman et al., 2014; Naylor et al., 2015; Townsend et al., 2017). Thus, KOPr agonists could be promising agents to prevent prescription opioid abuse whereby addition of KOPr agonists to existing opioids could reduce the addictive potential, allowing patients to receive effective pain relief without the development of dependence to drugs. Hence, it would be interesting to observe the effects of mixed opioid agonist like MP1104, with reduced side effects compared to pure KOPr agonist in these studies.

The emphasis made by current National Institutes of Health (NIH) guidelines on comparing the effects of pharmacotherapies in both genders, in order to improve the translation of animal studies into the clinic (Clayton and Collins, 2014), allows the future study of sex differences 
impacting the outcome of the anti-addictive and anti-pain studies conducted in this research. Studies have shown that analgesic effects in human and rodents vary between females and males (Rasakham and Liu-Chen, 2011; Wang et al., 2011). KOPr activating ligands such as pentazocine reported that females showed greater analgesic effects than males when investigated in a model of post-operative dental pain (Gear et al., 1996). In rodents, however KOPr agonists have been found to produce greater anti-nociceptive responses in males than females in thermal nociceptive pain models (Barrett et al., 2002; Sternberg et al., 2004). In chronic pain studies, women showed increased pain sensitivity and a greater prevalence of clinical pain (Mogil, 2012). The paclitaxelinduced neuropathic pain was measured in male and female mice in 10 inbred strains. Mechanical allodynia was measured using von Frey filaments, which found that males had significantly increased levels of allodynia for all the strains combined, but there was no significant effect of strain and sex interactions (Smith et al., 2004). Therefore, these variations of analgesic effects in sexes and species focus the need to study the effects of MP1104 on pain in female rodents as well. It is a future direction of this thesis research.

More recently, a novel beneficial role of KOPr activation has been highlighted. KOPr activation has been reported to promote oligodendrocyte differentiation and neuron remyelination, further KOPr activation has also shown to repair damaged neurons both in vivo and in vitro (Du et al., 2016; Mei et al., 2016). Another study showed that administration of paclitaxel caused myelin degeneration in C57BL/6 mice (Tasnim et al., 2016). Hence testing remyelination effects using our novel mixed KOPr/DOPr agonist in paclitaxel-administered mice would be interesting. For future studies, the sciatic nerve, lumbar spinal cord, and DRG tissue would ideally be collected at the end of chronic MP1104 administration in CINP and explored for remyelination properties. 


\subsubsection{KOPr co-administration prevents morphine-induced tolerance}

Morphine is efficient in treating pain clinically, however repeated used of morphine produces tolerance (Chu et al., 2006), a major side effect of this medication which hinders efficient treatment. Hence, there is a need to address this problem. Previously few studies have focussed on the scope of KOPr agonists in preventing development of tolerance to morphine analgesia (Suzuki et al., 1992a; Tao et al., 2008; Yamamoto et al., 1988). The results from these studies have been discussed briefly here. Yamamoto et al. (1988) reported that co-administration of traditional KOPr agonist U50,488 (3.2 mg/kg,i.p.) was shown to prevent the development of morphine tolerance and the anti-nociceptive effects of morphine were restored when U50,488 $(10 \mathrm{mg} / \mathrm{kg})$ was co-injected with morphine in morphine-tolerant male Wistar rats as measured using hotplate $\left(55^{\circ} \mathrm{C}\right)$ test (Yamamoto et al., 1988). Another study by Tao et al. (1994), using guinea pigs also showed that U50,488 (3 mg/kg, i.p.) partially restored morphine-induced anti-nociception in morphine tolerant $(8 \mathrm{mg} / \mathrm{kg} / \mathrm{day}$, i.p. for 6 days) guinea pigs, besides completely blocking the development of tolerance to morphine anti-nociception by co-administration of U50,488 at very low dose of $0.003 \mathrm{mg} / \mathrm{kg}$, i.p. Furthermore, the withdrawal signs induced by $8 \mathrm{mg} / \mathrm{kg}$ (i.p.) of naloxone hydrochloride on the seventh day were also depressed by co-administration of 0.003 $\mathrm{mg} / \mathrm{kg}$ U50,488 with morphine ( $8 \mathrm{mg} / \mathrm{kg}$ i.p.) every day for 7 days. As an additional support to these studies, the effects of selective KOPr antagonist, nor-BNI was examined on the development of tolerance to morphine analgesia and physical dependence on morphine in mice and rats. This study showed that the development of tolerance to morphine analgesia was significantly potentiated by pre-treatment of mice with nor-BNI $(5 \mathrm{mg} / \mathrm{kg}, \mathrm{s} . \mathrm{c}) 2 \mathrm{~h}$ prior to morphine treatment during chronic morphine treatment for five days (Suzuki et al., 1992a). This study also reported that nor-BNI pre-treatment during chronic treatment with the high dose of morphine for five days significantly potentiated the naloxone-induced body weight loss in morphine-dependent mice and rats (Suzuki et al., 1992a). These findings indicate that activation of the KOPr system blocks the development of tolerance to morphine analgesia. An important future direction of this study is to examine the effect of co-administration of MP1104 in morphine-tolerant rats and observe the effects produced thus focussing on the clinical implementation of MP1104. 


\subsubsection{Clinical translation}

Translating preclinical research findings to drugs that work in the clinic is problematic. Drug fail for many reasons and better models need to be developed to improve clinical translation. In this section, I would like to discuss few next steps needed to translate these preclinical research findings to benefit humans.

Conducting pharmacokinetic, pharmacodynamics and toxicology studies is essential for any drug (chemical compound derived synthetically or naturally) with a therapeutically beneficial value. It is important to note here that MP1104 has not been screened for these studies in rodents previously. One of the primary and most critical step for clinical translation of MP1104 will be carrying out extensive clinical pharmacokinetic, pharmacodynamics and toxicology studies. Pharmacokinetic studies provides information about drug absorption, distribution, metabolism, and excretion (Ruiz-Garcia et al., 2008), whereas pharmacodynamics studies highlight the physiological and biochemical effects of drug in the body via receptor binding and activation (Lees et al., 2004). Toxicology studies on the other hand throw insight on the adverse effects of drugs in the body (Lee et al., 2017).

Developing rodent models of CIPN which replicate all the symptoms that patients report is challenging because numbness, tingling and ongoing/spontaneous pain all rely on verbal report from the patient. Thus, most preclinical studies have focused on measuring evoked painlike behaviours, and investigations into novel measures of spontaneous pain in CIPN rodents are ongoing (Flatters et al., 2017). Spontaneous pain can be measured in animals using tests such as weight bearing, which measures the weight distribution of paws, with a view towards representing the change in hind-paw weight distribution between the affected and unaffected hind limb or hind-paw (Combe et al., 2004; Kim et al., 2015). Alternately, ultrasound vocalization recording, which records vocalizations of rodents in pain in an unspecific sound-free environment, can also be used (Han et al., 2005; Kurejova et al., 2010) (for review on the models to measure spontaneous pain refer to Tappe-Theodor and Kuner, 2014). Therefore, measuring spontaneous pain with an established method in animals might allow easy translation of MP1104 for clinical use. On this note, it is worthwhile to remind that MP1104 did not induce tolerance after repeated administration in CINP mice model (Figure 3.8A \& B), unlike the clinically available MOPr analgesic morphine (Chu et al., 2006), or gabapentin (Kivell Lab unpublished data). Therefore, MP1104 shows preclinical improvements over the clinically utilised CINP medication, gabapentin. 


\subsection{Final conclusions}

This thesis explores the anti-cocaine and anti-nociceptive effects of MP1104, a potent novel KOPr and DOPr agonist. MP1104 shows anti-cocaine effects in a self-administration model of relapse and in cocaine dose-response self-administration tests in male Sprague-Dawley rats. The anticocaine effects observed in self-administration studies are at least in part due to KOPr-mediated enhancement of DAT activity, as increased uptake of DA by DAT is observed in dStr and NAc rat brain tissues treated with MP1104.

There is a demand for developing non-addictive analgesics due to the increasing number of deaths globally due to misuse of prescription opioids. Hence, MP1104 was screened for anti-nociceptive effects. MP1104 shows anti-nociceptive effects to thermal nociceptive pain with longer duration of action compared to the clinically prescribed analgesic morphine. In the paclitaxel-induced neuropathic pain model, MP1104, in contrast to morphine, showed potent anti-nociceptive effects with no observed tolerance. Additionally, MP1104 was evaluated for side effects such as sedation, anxiety, aversion and pro-depressive effects using spontaneous locomotor activity, EPM, CPA and FST respectively. In these tests, MP1104 showed reduced side effects. This is a first study to show that DOPr actions of MP1104 attenuates the aversive KOPr mediated effects of MP1104, indicating the advantage of dual KOPr/DOPr agonists to be developed into effective pharmacotherapies for addiction and analgesia with reduced side effects compared to selective KOPr agonists which are reported to show profound adverse effects limiting their clinical development. 


\section{Appendices}

\section{Appendix A: Solutions}

KREBS Buffer $\mathrm{pH} 7.4$

Concentration (M) g/L

$\begin{array}{lll}\mathrm{NaCl} & 130 \mathrm{mM} & 7.6 \\ \mathrm{KCl} & 1.3 \mathrm{mM} & 0.10 \\ \mathrm{CaCl}_{2} & 2.2 \mathrm{mM} & 0.24 \\ \mathrm{MgSO}_{4} .6 \mathrm{H}_{2} \mathrm{O} & 1.2 \mathrm{mM} & 0.27 \\ \mathrm{KH}_{2} \mathrm{PO}_{4} & 1.2 \mathrm{mM} & 0.16 \\ \mathrm{HEPES} & 10 \mathrm{mM} & 2.6 \\ \text { D-glucose } & 10 \mathrm{mM} & 1.8\end{array}$




\section{Appendix B: Determination of differences between drug naïve and U50,488 agonist pre-treated rats}

Preliminary data $(n=2-8)$ from previous research carried out in the Kivell Laboratory showed that rats pre-treated with KOPr agonists: U50, 488 (10 mg/kg, i.p.), Sal A (1 mg/kg, i.p.) and Mesyl Sal B $(0.3 \mathrm{mg} / \mathrm{kg}$, i.p) a week prior to surgery (to insert indwelling jugular catheters) took longer to acquire cocaine self-administration behaviour compared to drug naïve rats (Ewald, 2015). Therefore, this experiment aimed to fully evaluate this preliminary finding to determine whether pre-treatment of rats with KOPr agonist prolongs acquisition of cocaine self-administration behaviour. Male Sprague-Dawley rats ( $n=10-11$ /group) were divided into two groups, one group received the KOPr agonist U50,488 (10 mg/kg, i.p.) and the other group were vehicle-treated control rats. A week later rats underwent surgery for implantation of catheter and were trained to self-administer cocaine post recovery. Control rats acquired cocaine self-administration with a mean acquisition time of $6 \pm 2$ days (mean \pm SEM), whereas, the U50,488 pre-treated rats showed a mean acquisition time of $11 \pm 1$ day (Figure B.1A \& B). The data showed was not statistically significant ( $p>0.999$, Kolmogorov-Smirnov nonparametric test; $p=0.067$, Kaplan meier method), however, the overall results showed a trend towards delayed acquisition time in rats pre-treated with U50,488 compared to drug naïve rats. 
A

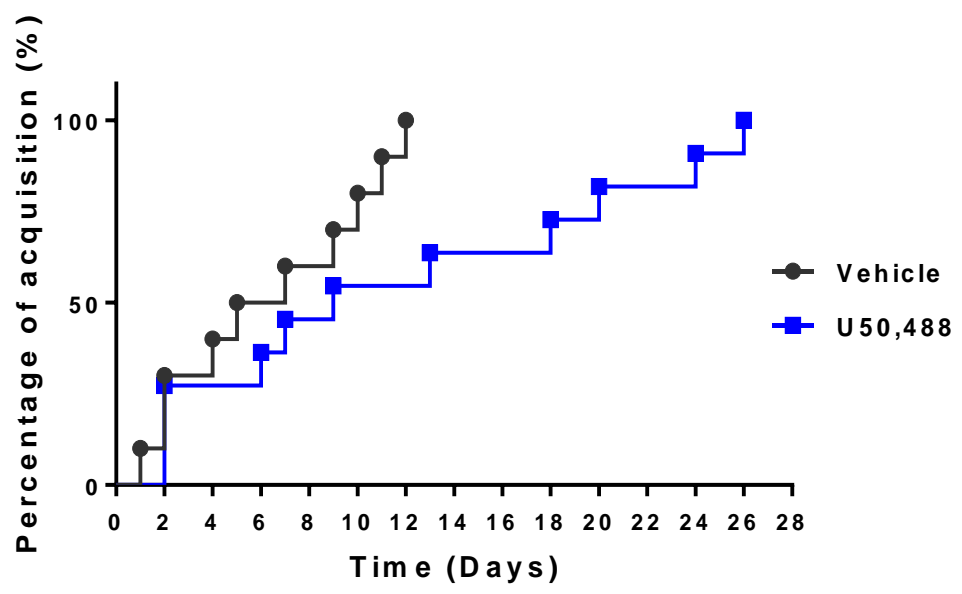

B

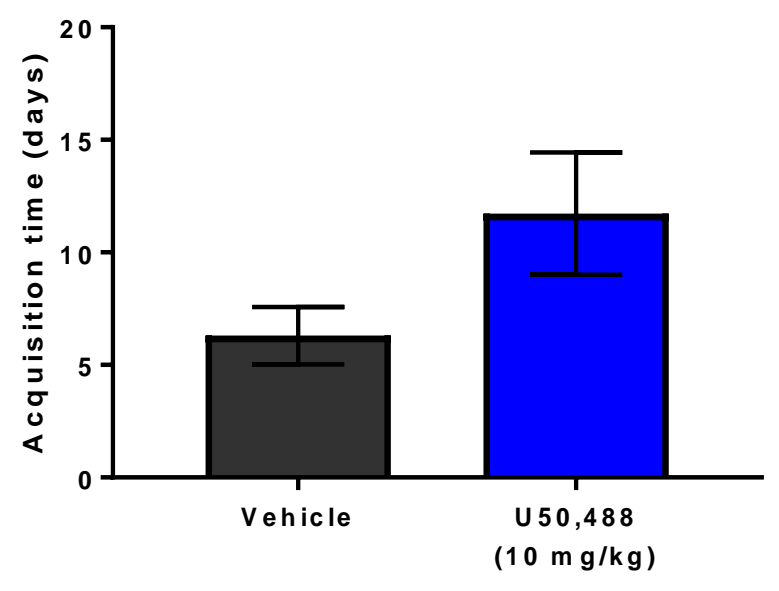

Figure B.1: KOPr pre-treatment effects in cocaine self-administration acquisition time in rats

(A) Drug naïve rats achieved acquisition by day 6 , whereas rats pre-treated with traditional KOPr agonist, U50,488 achieved the acquisition criteria by day 11 . (B) Rats pre-treated with U50,488 showed a non-significant increase in acquisition time compared to drugs naïve rats. Student's $t$ test. Values expressed as mean \pm SEM, $n=10$-11 per group. 


\section{Appendix C: Latin square treatment order for drug self-administration study}

For drug self-administration experiments, where subjects received multiple treatments, a Latin square counterbalanced treatment order was used.

\section{C.1 Cocaine reinstatement treatments}

Table C.1: Treatment design used in cocaine-induced reinstatement of drug seeking

\begin{tabular}{|l|l|l|l|l|l|l|}
\hline Rat no. & Session 1 & Session 2 & Session 3 & Session 4 & Session 5 & Session 6 \\
\hline 1 & $0.3 \mathrm{MP1104}$ & Vehicle & $1.0 \mathrm{MP1104}$ & $\begin{array}{l}1.0 \mathrm{MP1104} \\
+\mathrm{NTI}\end{array}$ & $\begin{array}{l}1.0 \mathrm{MP1104} \\
+ \text { nor-BNI }\end{array}$ & $\begin{array}{l}1.0 \mathrm{MP1104}+ \\
\mathrm{NTI}+\text { nor-BNI }\end{array}$ \\
\hline 2 & Vehicle & $1.0 \mathrm{MP1104}$ & $0.3 \mathrm{MP1104}$ & $\begin{array}{l}1.0 \mathrm{MP1104} \\
+\mathrm{NTI}\end{array}$ & $\begin{array}{l}1.0 \mathrm{MP1104} \\
+ \text { nor-BNI }\end{array}$ & $\begin{array}{l}1.0 \mathrm{MP1104}+ \\
\mathrm{NTI}+\text { nor-BNI }\end{array}$ \\
\hline 3 & $1.0 \mathrm{MP1104}$ & $0.3 \mathrm{MP1104}$ & Vehicle & $\begin{array}{l}1.0 \mathrm{MP1104} \\
+\mathrm{NTI}\end{array}$ & $\begin{array}{l}1.0 \mathrm{MP1104} \\
+ \text { nor-BNI }\end{array}$ & $\begin{array}{l}1.0 \mathrm{MP1104}+ \\
\mathrm{NTI}+\text { nor-BNI }\end{array}$ \\
\hline 4 & $1.0 \mathrm{MP1104}$ & Vehicle & $0.3 \mathrm{MP1104}$ & $\begin{array}{l}1.0 \mathrm{MP1104} \\
+\mathrm{NTI}\end{array}$ & $\begin{array}{l}1.0 \mathrm{MP1104} \\
+ \text { nor-BNI }\end{array}$ & $\begin{array}{l}1.0 \mathrm{MP1104}+ \\
\mathrm{NTI}+\text { nor-BNI }\end{array}$ \\
\hline 5 & Vehicle & $0.3 \mathrm{MP1104}$ & $1.0 \mathrm{MP1104}$ & $\begin{array}{l}1.0 \mathrm{MP1104} \\
+\mathrm{NTI}\end{array}$ & $\begin{array}{l}1.0 \mathrm{MP1104} \\
+ \text { nor-BNI }\end{array}$ & $\begin{array}{l}1.0 \mathrm{MP1104}+ \\
\mathrm{NTI}+\text { nor-BNI }\end{array}$ \\
\hline 6 & $0.3 \mathrm{MP1104}$ & $1.0 \mathrm{MP1104}$ & Vehicle & $\begin{array}{l}1.0 \mathrm{MP1104} \\
+\mathrm{NTI}\end{array}$ & $\begin{array}{l}1.0 \mathrm{MP1104} \\
+ \text { nor-BNI }\end{array}$ & $\begin{array}{l}1.0 \mathrm{MP1104}+ \\
\mathrm{NTI}+\text { nor-BNI }\end{array}$ \\
\hline 7 & $0.3 \mathrm{MP1104}$ & Vehicle & $1.0 \mathrm{MP1104}$ & $\begin{array}{l}1.0 \mathrm{MP1104} \\
+\mathrm{NTI}\end{array}$ & $\begin{array}{l}1.0 \mathrm{MP1104} \\
+ \text { nor-BNI }\end{array}$ & $\begin{array}{l}1.0 \mathrm{MP1104}+ \\
\mathrm{NTI}+\text { nor-BNI }\end{array}$ \\
\hline 8 & Vehicle & $1.0 \mathrm{MP1104}$ & $0.3 \mathrm{MP1104}$ & $\begin{array}{l}1.0 \mathrm{MP1104} \\
+\mathrm{NTI}\end{array}$ & $\begin{array}{l}1.0 \mathrm{MP1104} \\
+ \text { nor-BNI }\end{array}$ & $\begin{array}{l}1.0 \mathrm{MP1104}+ \\
\mathrm{NTI}+\text { nor-BNI }\end{array}$ \\
\hline 9 & $1.0 \mathrm{MP1104}$ & $0.3 \mathrm{MP1104}$ & Vehicle & $\begin{array}{l}1.0 \mathrm{MP1104} \\
+\mathrm{NTI}\end{array}$ & $\begin{array}{l}1.0 \mathrm{MP1104} \\
+ \text { nor-BNI }\end{array}$ & $\begin{array}{l}1.0 \mathrm{MP1104}+ \\
\mathrm{NTI}+\text { nor-BNI }\end{array}$ \\
\hline
\end{tabular}

Treatment order for rats with MP1104 $(n=9)$. Numbers represent doses used (example: $0.3=0.3$ $\mathrm{mg} / \mathrm{kg}$ ). Vehicle = DMSO:Tween-80:MilliQ water in a ratio of 2:1:7. All vehicle and MP1104 agonist were given i.p., whereas NTI and nor-BNI were given s.c. 


\section{C.2 Dose-response cocaine self-administration test treatments}

Table C.2: Treatment design used in dose-response cocaine self-administration test

\begin{tabular}{|l|l|l|l|}
\hline Rat no. & Session 1 & Session 2 & Session 3 \\
\hline 1 & Vehicle & $0.3 \mathrm{MP1104}$ & $0.6 \mathrm{MP1104}$ \\
\hline 2 & $0.3 \mathrm{MP1104}$ & $0.6 \mathrm{MP1104}$ & Vehicle \\
\hline 3 & $0.6 \mathrm{MP1104}$ & Vehicle & $0.3 \mathrm{MP1104}$ \\
\hline 4 & Vehicle & $0.6 \mathrm{MP1104}$ & $0.3 \mathrm{MP1104}$ \\
\hline 5 & $0.6 \mathrm{MP1104}$ & $0.3 \mathrm{MP1104}$ & Vehicle \\
\hline 6 & $0.3 \mathrm{MP1104}$ & Vehicle & $0.6 \mathrm{MP1104}$ \\
\hline
\end{tabular}

Latin square design treatment order for rats with MP1104 $(n=6)$. Numbers represent doses used (example: $0.3=0.3 \mathrm{mg} / \mathrm{kg}$ ). Vehicle = DMSO:Tween-80:MilliQ water in a ratio of 2:1:7. All treatments were administered via i.p. injections. 


\section{Appendix D: Warm-water tail withdrawal dose-response experiment}

As a control for the cumulative dose-response tail withdrawal assay, rats (A) and mice (B) were administered an equivalent number of vehicle injections. The graphs display vehicle control showed no anti-nociceptive effect with no increase in effect over time indicating that the results shown by MP1104 agonist were neither due to sedative effects or stress-induced analgesia. 
A

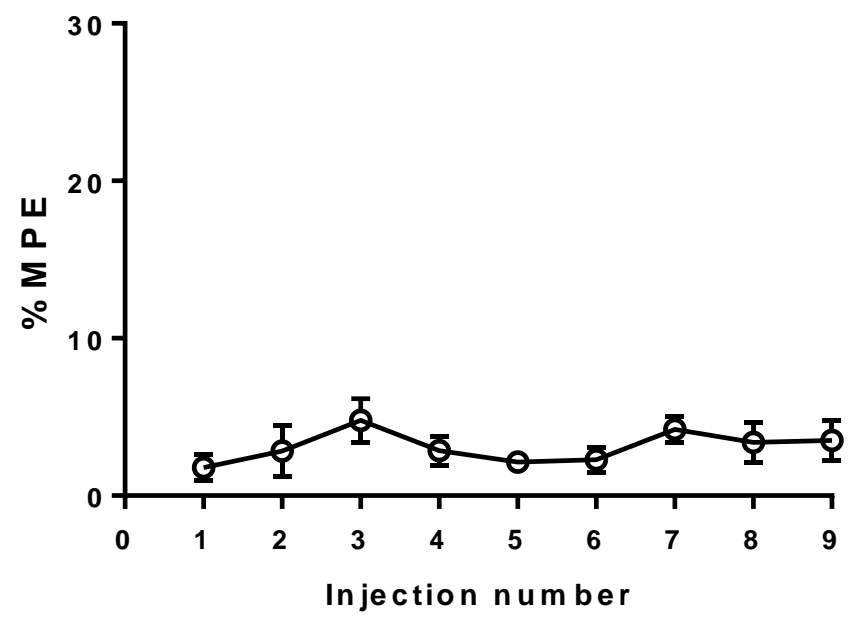

B

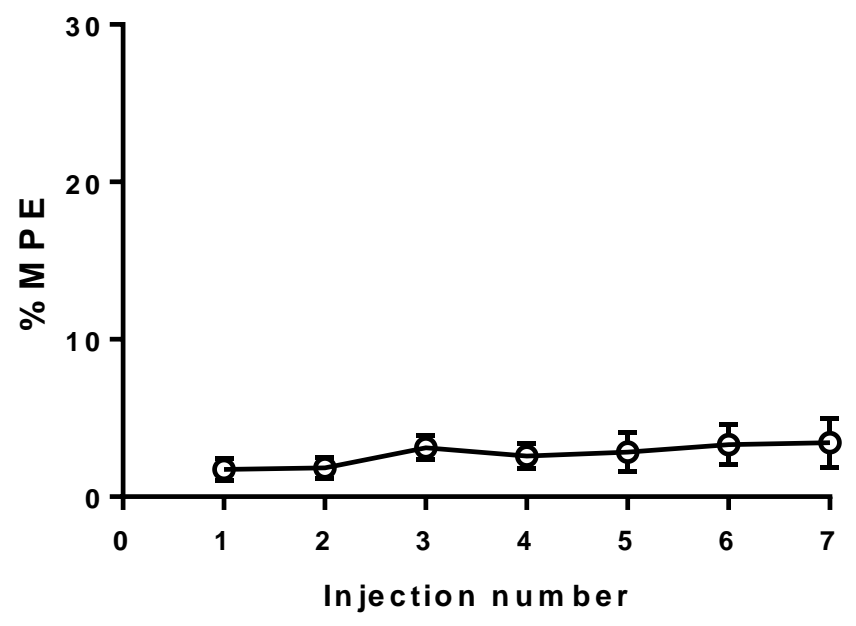

Figure D.1: Vehicle control group in the warm-water tail withdrawal dose-response assay

Administration of s.c. injections of vehicle in (A) rats and (B) mice in the same number as MP1104 agonist treatment. One-way ANOVA repeated measures showed no significant effect between the successive injections $\left(F_{(3.4,9.7)}=1.55, p=0.515\right.$ for rats; $F_{(6.1,11.3)}=, p=0.781$, for mice). Values expressed as mean \pm SEM, $n=5-6$. 


\section{Appendix E: Testing scheme of the simplified up-down von Frey filament}

Filament no.

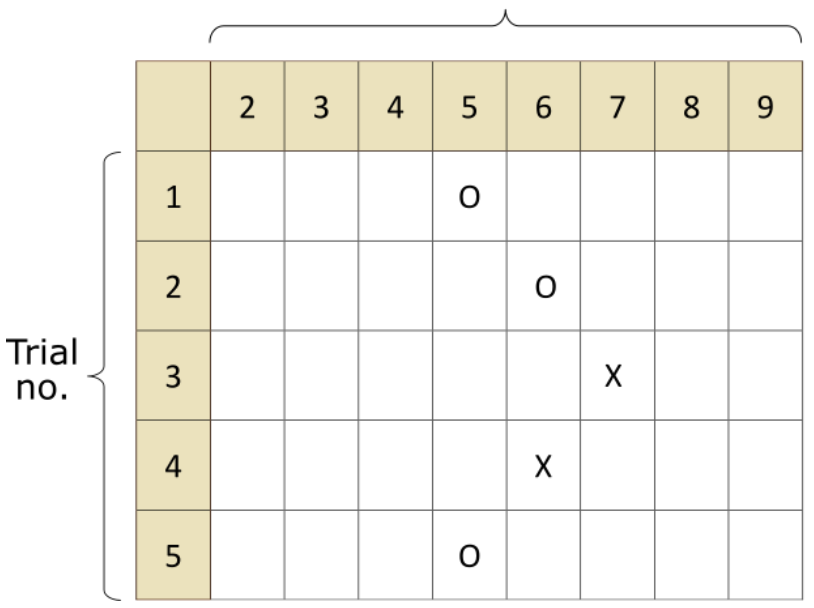

\begin{tabular}{|l|l|l|l|l|l|l|l|l|}
\hline & 2 & 3 & 4 & 5 & 6 & 7 & 8 & 9 \\
\hline 1 & & & & 0 & & & & \\
\hline 2 & & & & & 0 & & & \\
\hline 3 & & & & & & 0 & & \\
\hline 4 & & & & & & & 0 & \\
\hline 5 & & & & & & & & $x$ \\
\hline
\end{tabular}

\section{Figure E.1: Examples of the simplified up-down von Frey filament testing scheme}

Two examples of the simplified up-down method using von Frey filaments to test for mechanical allodynia. The testing always begins with filament number 5 . No withdrawal response $(0)$ means the next highest filament is subsequently used. A positive withdrawal response $(X)$ means that the next lowest filament is used next. The withdrawal scores are 5.5 and 8.5 for these two examples. 


\section{Appendix F: Acute dose-response controls in the paclitaxel-induced neuropathic pain model}

\section{F.1 Baseline withdrawal thresholds}

The baseline (day 0) values were compared between the designated treatment groups assigned for the dose-response experiment.

A

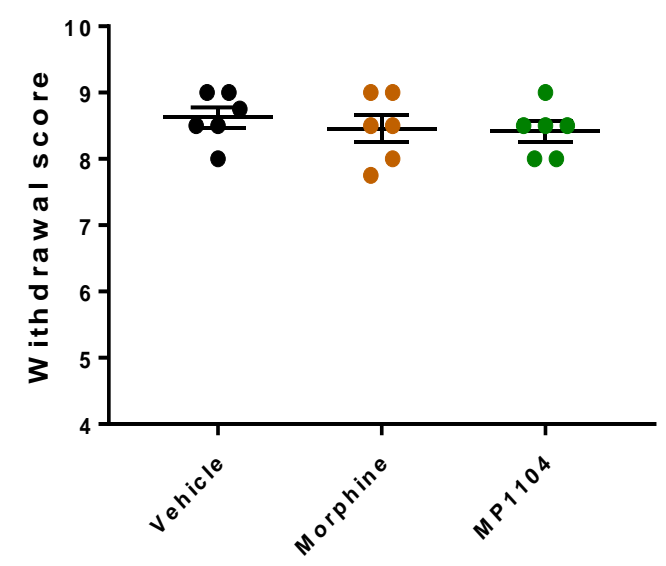

B

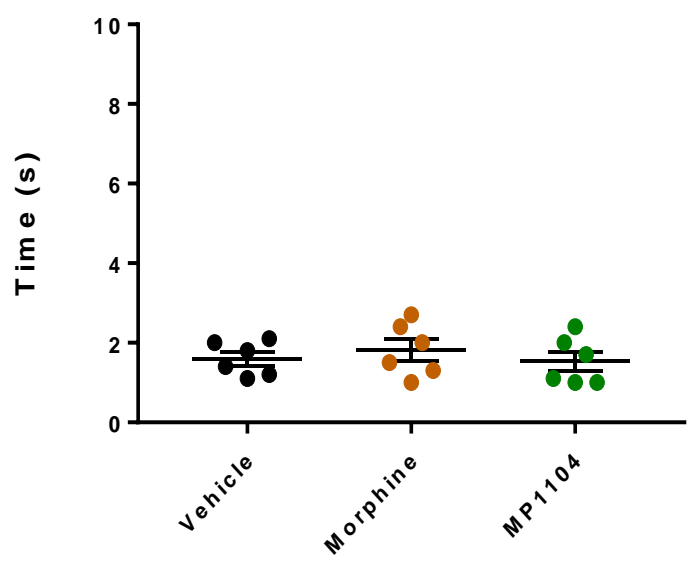

Figure F.1: Day 0 pre-paclitaxel mechanical and cold threshold values showed no differences between the assigned dose-response treatment groups

On day 0 baseline values (prior to the first administration of paclitaxel) measured for (A) mechanical and (B) cold allodynia in mice showed no significant differences between the assigned treatment group. Values expressed as individual data points, with a line indicating the mean \pm SEM, $n=6$. 


\section{F.2 Day 15 pre-treatment withdrawal thresholds}

The day 15 values were compared depending on the assigned treatment in the dose-response procedure.

A

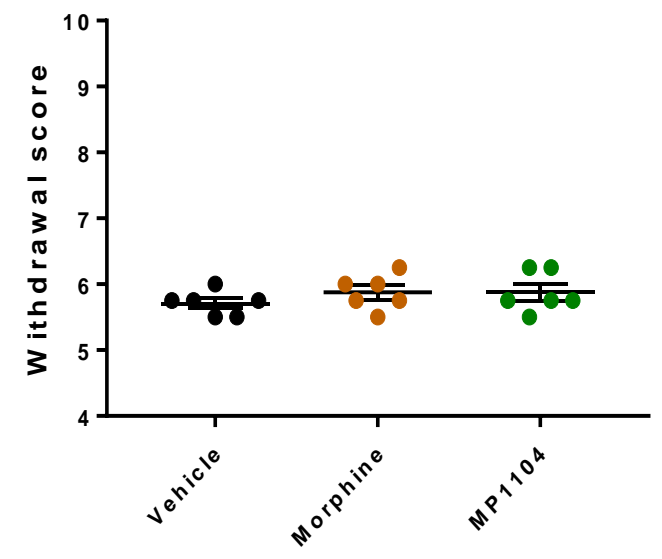

B

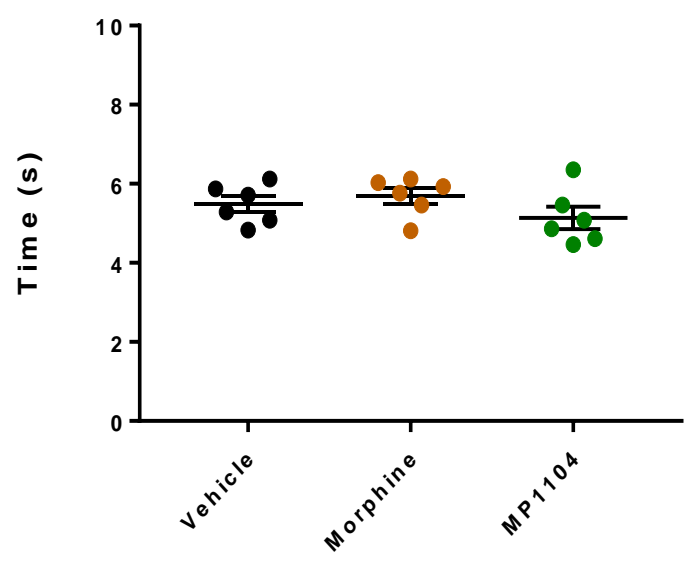

Figure F.2: Day 15 post-paclitaxel mechanical and cold allodynia values showed no differences between the assigned dose-response treatment groups

On day 15, post-paclitaxel treated values measured in mice for (A) mechanical and (B) cold allodynia, showed no significant differences between the assigned treatment group. Values expressed as individual data points, with a line indicating the mean $\pm S E M, n=6$. 


\section{F.3 Vehicle treatment in the dose-response method}

An equivalent number of vehicle treatments were given to paclitaxel-treated mice for comparison in the dose-response procedure. The graphs displayed vehicle-treated mice had no antinociceptive effect and successive injections did not have any effects over time.

A

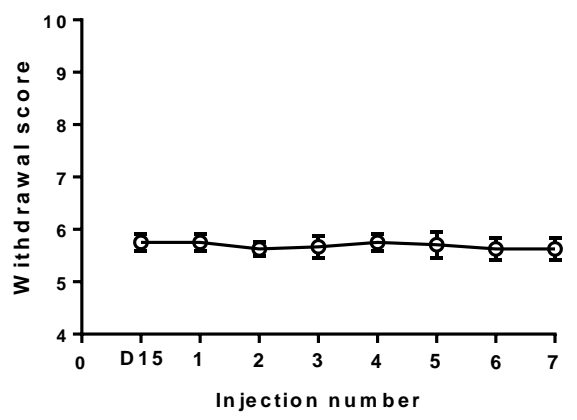

B

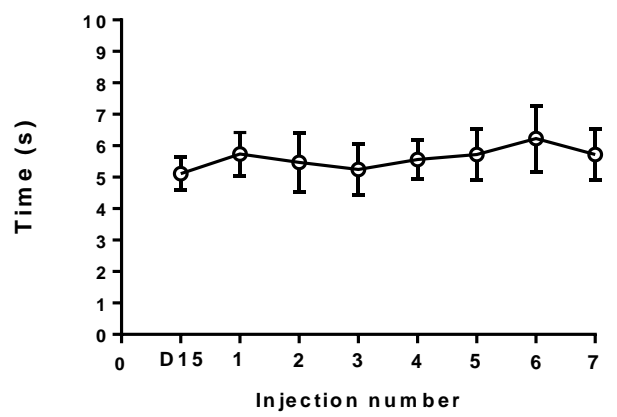

Figure F.3: In mice with established paclitaxel-induced neuropathic pain vehicle treatment did not have an anti-nociceptive effect

Vehicle controls were administered to mice with paclitaxel-induced neuropathic pain on day 15 , with the same number of injections as MP1104 or morphine. There was no significant effect of vehicle over time for $(A)$ mechanical allodynia $\left(F_{(5,41)}=1.62, p=0.758\right)$ and for $(B)$ cold allodynia $\left(F_{(5,41)}=1.207, p=0.821\right)$. One-way repeated measures ANOVA. D15 refers to the values on day 15 prior to vehicle treatment. Values expressed as mean $\pm S E M, n=6$. 


\section{Appendix G: Chronic administration procedure controls in the paclitaxel- induced neuropathic pain model}

The baseline values on day 15 were compared between the assigned treatment groups in the paclitaxel-treated animals before the initiation of the daily treatment on day 16.

A

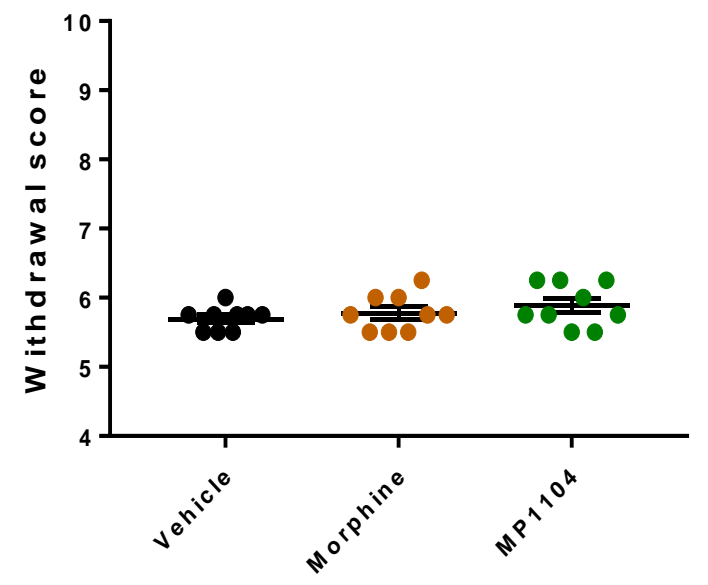

B

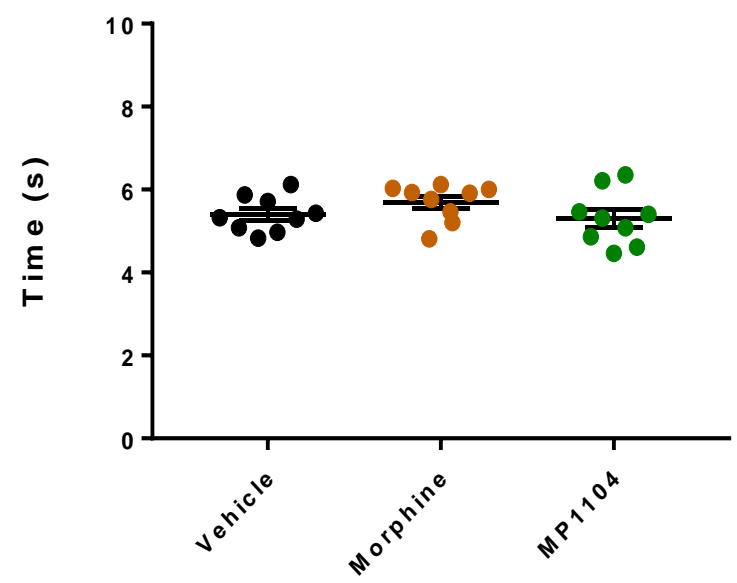

Figure G.1: Mechanical withdrawal thresholds and cold response times on day 15 before assignment of treatment groups

(A) The mechanical withdrawal and (B) cold response time values on day 15 were not significantly different when separated by the assigned treatment groups. Values expressed as individual data points, with a line indicating the mean $\pm S E M, n=9$. 


\section{Appendix H: Publications from this PhD study}

Atigari, D. V., Uprety, R., Pasternak, G. W., Majumdar, S., \& Kivell, B. M. 2019. MP1104, a mixed kappa-delta opioid receptor agonist has anti-cocaine properties with reduced side-effects in rats. Neuropharmacology. 


\section{References}

Aceto, M. D., Harris, L. S., Negus, S. S., Banks, M. L., Hughes, L. D., Akgün, E., \& Portoghese, P. S. 2012. MDAN-21: a bivalent opioid ligand containing mu-agonist and delta-antagonist pharmacophores and its effects in rhesus monkeys. International journal of medicinal chemistry, 2012.

Adinoff, B. 2004. Neurobiologic processes in drug reward and addiction. Harvard review of psychiatry, 12(6), 305-320.

Akiyama, T., CArSTENS, M. I., Piecha, D., Steppan, S., \& CArSTENS, E. 2015. Nalfurafine suppresses pruritogen-and touch-evoked scratching behavior in models of acute and chronic itch in mice. Acta dermato-venereologica, 95(2), 147-150.

Al-Hasani, R., McCall, J. G., Shin, G., Gomez, A. M., Schmitz, G. P., Bernardi, J. M., . . Bruchas, M. R. 2015. Distinct Subpopulations of Nucleus Accumbens Dynorphin Neurons Drive Aversion and Reward. Neuron, 87(5), 1063-1077. doi:10.1016/j.neuron.2015.08.019

Altshuler, H. L., Phillips, P. E., \& Feinhandler, D. A. 1980. Alteration of ethanol self-administration by naltrexone. Life sciences, 26(9), 679-688.

Ambrose-Lanci, L. M., Sterling, R., \& Van Bockstaele, E. J. 2010. Cocaine withdrawal-induced anxiety in females: Impact of circulating estrogen and potential use of delta-opioid receptor agonists for treatment. Journal of neuroscience research, 88(4), 816-824.

Anand, J. P., Boyer, B. T., Mosberg, H. I., \& Jutkiewicz, E. M. 2016. The behavioral effects of a mixed efficacy antinociceptive peptide, VRP26, following chronic administration in mice. Psychopharmacology, 233(13), 2479-2487.

Anand, J. P., \& Montgomery, D. 2018. Multifunctional Opioid Ligands.

Ananthan, S. 2006. Opioid ligands with mixed $\mu / \delta$ opioid receptor interactions: an emerging approach to novel analgesics. The AAPS journal, 8(1), E118-E125.

Anderson, B. A., \& Yantis, S. 2013. Persistence of value-driven attentional capture. Journal of Experimental Psychology: Human Perception and Performance, 39(1), 6.

Atigari, D. V., Uprety, R., Pasternak, G. W., Majumdar, S., \& Kivell, B. M. 2019. MP1104, a mixed kappa-delta opioid receptor agonist has anti-cocaine properties with reduced side-effects in rats. Neuropharmacology, 150, 217-228.

Avidor-Reiss, T., Zippel, R., Levy, R., Saya, D., Ezra, V., Barg, J., . . . Vogel, Z. 1995. k-Opioid receptortransfected cell lines: modulation of adenylyl cyclase activity following acute and chronic opioid treatments. Febs Letters, 361(1), 70-74.

Balboni, G., Guerrini, R., Salvadori, S., Bianchi, C., Rizzi, D., Bryant, S. D., \& Lazarus, L. H. 2002. Evaluation of the Dmt- Tic Pharmacophore: Conversion of a Potent $\delta$-Opioid Receptor Antagonist into a Potent $\delta$ Agonist and Ligands with Mixed Properties. Journal of medicinal chemistry, 45(3), 713-720.

Balster, R. L., \& Schuster, C. R. 1973. A comparison of d-amphetamine, l-amphetamine, and methamphetamine self-administration in rhesus monkeys. Pharmacology Biochemistry and Behavior, 1(1), 67-71.

Barber, A., Bartoszyk, G., Bender, H., Gottschlich, R., Greiner, H., Harting, J., . . Simon, M. 1994. A pharmacological profile of the novel, peripherally-selective k-opioid receptor agonist, EMD 61753. British journal of pharmacology, 113(4), 1317-1327.

Baron, R., Binder, A., \& Wasner, G. 2010. Neuropathic pain: diagnosis, pathophysiological mechanisms, and treatment. The Lancet Neurology, 9(8), 807-819. 
Barrett, A. C., Cook, C. D., Terner, J. M., Roach, E. L., Syvanthong, C., \& Picker, M. J. 2002. Sex and rat strain determine sensitivity to $\mathrm{k}$ opioid-induced antinociception. Psychopharmacology, 160(2), 170-181.

Beardsley, P. M., Howard, J. L., Shelton, K. L., \& Carroll, F. I. 2005. Differential effects of the novel kappa opioid receptor antagonist, JDTic, on reinstatement of cocaine-seeking induced by footshock stressors vs cocaine primes and its antidepressant-like effects in rats. Psychopharmacology, 183(1), 118.

Beaudry, H., Gendron, L., \& Morón, J. A. 2015. Implication of delta opioid receptor subtype 2 but not delta opioid receptor subtype 1 in the development of morphine analgesic tolerance in a rat model of chronic inflammatory pain. European Journal of Neuroscience, 41(7), 901907.

Béguin, C., Duncan, K. K., Munro, T. A., Ho, D. M., Xu, W., Liu-Chen, L.-Y., . . Cohen, B. M. 2009. Modification of the furan ring of salvinorin $A$ : identification of a selective partial agonist at the kappa opioid receptor. Bioorganic \& medicinal chemistry, 17(3), 1370-1380.

Belcheva, M. M., Clark, A. L., Haas, P. D., Serna, J. S., Hahn, J. W., Kiss, A., \& Coscia, C. J. 2005. $\mu$ and $\mathrm{K}$ opioid receptors activate ERK/MAPK via different protein kinase $\mathrm{C}$ isoforms and secondary messengers in astrocytes. Journal of Biological Chemistry, 280(30), 2766227669.

Belin, D., Jonkman, S., Dickinson, A., Robbins, T. W., \& Everitt, B. J. 2009. Parallel and interactive learning processes within the basal ganglia: relevance for the understanding of addiction. Behavioural brain research, 199(1), 89-102.

Belkaï, E., Scherrmann, J. M., Noble, F., \& Marie-Claire, C. 2009. PRECLINICAL STUDY: Modulation of MDMA-induced behavioral and transcriptional effects by the delta opioid antagonist naltrindole in mice. Addiction biology, 14(3), 245-252.

Bennett, G. J., \& Xie, Y.-K. 1988. A peripheral mononeuropathy in rat that produces disorders of pain sensation like those seen in man. Pain, 33(1), 87-107.

Berridge, K. C., \& Robinson, T. E. 1995. The mind of an addicted brain: neural sensitization of wanting versus liking. Current Directions in Psychological Science, 4(3), 71-75.

Berridge, K. C., \& Robinson, T. E. 1998. What is the role of dopamine in reward: hedonic impact, reward learning, or incentive salience? Brain research reviews, 28(3), 309-369.

Berridge, K. C., \& Kringelbach, M. L. 2015. Pleasure systems in the brain. Neuron, 86(3), 646-664.

Berterame, S., Erthal, J., Thomas, J., Fellner, S., Vosse, B., Clare, P., . . Pavadia, J. 2016. Use of and barriers to access to opioid analgesics: a worldwide, regional, and national study. The Lancet, 387(10028), 1644-1656.

Bhushan, R. G., Sharma, S. K., Xie, Z., Daniels, D. J., \& Portoghese, P. S. 2004. A Bivalent Ligand (KDN-21) Reveals Spinal $\delta$ and $к$ Opioid Receptors Are Organized as Heterodimers That Give Rise to $\delta 1$ and $\mathrm{k} 2$ Phenotypes. Selective Targeting of $\delta-\mathrm{k}$ Heterodimers. Journal of medicinal chemistry, 47(12), 2969-2972.

Bidlack, J. M. 2014. Mixed kappa/mu partial opioid agonists as potential treatments for cocaine dependence. Adv Pharmacol, 69, 387-418.

Bie, B., \& Pan, Z. Z. 2007. Trafficking of central opioid receptors and descending pain inhibition. Molecular Pain, 3(1), 37.

Bileviciute-Ljungar, I., Saxne, T., \& Spetea, M. 2005. Anti-inflammatory effects of contralateral administration of the $\mathrm{k}$-opioid agonist $\mathrm{U}-50,488 \mathrm{H}$ in rats with unilaterally induced adjuvant arthritis. Rheumatology, 45(3), 295-302.

Bilsky, E. J., Calderon, S. N., Wang, T., Bernstein, R. N., Davis, P., Hruby, V. J., . . Porreca, F. 1995. SNC 80, a selective, nonpeptidic and systemically active opioid delta agonist. Journal of Pharmacology and Experimental Therapeutics, 273(1), 359-366. 
Binder, W., Machelska, H., Mousa, S., Schmitt, T., Rivière, P. J., Junien, J.-L., . . Schäfer, M. 2001. Analgesic and antiinflammatory effects of two novel k-opioid peptides. Anesthesiology: The Journal of the American Society of Anesthesiologists, 94(6), 1034-1044.

Bohn, L. M., Gainetdinov, R. R., Lin, F.-T., Lefkowitz, R. J., \& Caron, M. G. 2000. $\mu$-Opioid receptor desensitization by $\beta$-arrestin-2 determines morphine tolerance but not dependence. Nature, 408(6813), 720.

Boileau, I., Dagher, A., Leyton, M., Gunn, R. N., Baker, G. B., Diksic, M., \& Benkelfat, C. 2006. Modeling sensitization to stimulants in humans: an [11C] raclopride/positron emission tomography study in healthy men. Archives of general psychiatry, 63(12), 1386-1395.

Boja, J. W., \& Kuhar, M. J. 1989. [3H] Cocaine binding and inhibition of [3H] dopamine uptake is similar in both the rat striatum and nucleus accumbens. European journal of pharmacology, 173(2-3), 215-217.

Bolan, E. A., Kivell, B., Jaligam, V., Oz, M., Jayanthi, L. D., Han, Y., ... Devi, L. A. 2007. D2 receptors regulate dopamine transporter function via an extracellular signal-regulated kinases 1 and 2-dependent and phosphoinositide 3 kinase-independent mechanism. Molecular pharmacology, 71(5), 1222-1232.

Bonano, J. S., Runyon, S. P., Hassler, C., Glennon, R. A., \& Negus, S. S. 2014. Effects of the neuropeptide $S$ receptor antagonist RTI-118 on abuse-related facilitation of intracranial self-stimulation produced by cocaine and methylenedioxypyrovalerone (MDPV) in rats. European journal of pharmacology, 743, 98-105.

Bonese, K., Wainer, B., Fitch, F., Rothberg, R., \& Schuster, C. 1974. Changes in heroin selfadministration by a rhesus monkey after morphine immunisation. Nature, 252(5485), 708.

Bonin, R. P., Bories, C., \& De Koninck, Y. 2014. A simplified up-down method (SUDO) for measuring mechanical nociception in rodents using von Frey filaments. Molecular pain, 10(1), 26.

Bossert, J. M., Marchant, N. J., Calu, D. J., \& Shaham, Y. 2013. The reinstatement model of drug relapse: recent neurobiological findings, emerging research topics, and translational research. Psychopharmacology, 229(3), 453-476.

Bourdonnec, B. L., Windh, R. T., Ajello, C. W., Leister, L. K., Gu, M., Chu, G.-H., . . Wiant, D. D. 2008. Potent, orally bioavailable delta opioid receptor agonists for the treatment of pain: discovery of N, N-diethyl-4-(5-hydroxyspiro [chromene-2, 4'-piperidine]-4-yl) benzamide (ADL5859). Journal of medicinal chemistry, 51(19), 5893-5896.

Bourne, S., Machado, A. G., \& Nagel, S. J. 2014. Basic anatomy and physiology of pain pathways. Neurosurgery Clinics, 25(4), 629-638.

Bowen, C. A., Negus, S. S., Zong, R., Neumeyer, J. L., Bidlack, J. M., \& Mello, N. K. 2003a. Effects of mixed-action $\mathrm{k} / \mu$ opioids on cocaine self-administration and cocaine discrimination by rhesus monkeys. Neuropsychopharmacology.

Bowen, C. A., Negus, S. S., Zong, R., Neumeyer, J. L., Bidlack, J. M., \& Mello, N. K. 2003b. Effects of mixed-action $\mathrm{k} / \mu$ opioids on cocaine self-administration and cocaine discrimination by rhesus monkeys. Neuropsychopharmacology, 28(6), 1125.

Braida, D., Capurro, V., Zani, A., Rubino, T., Viganò, D., Parolaro, D., \& Sala, M. 2009. Potential anxiolytic-and antidepressant-like effects of salvinorin $A$, the main active ingredient of Salvia divinorum, in rodents. British journal of pharmacology, 157(5), 844-853.

Brainin-Mattos, J., Smith, N. D., Malkmus, S., Rew, Y., Goodman, M., Taulane, J., \& Yaksh, T. L. 2006. Cancer-related bone pain is attenuated by a systemically available $\delta$-opioid receptor agonist. Pain, 122(1-2), 174-181.

Breivik, H., Collett, B., Ventafridda, V., Cohen, R., \& Gallacher, D. 2006. Survey of chronic pain in Europe: prevalence, impact on daily life, and treatment. European journal of pain, 10(4), 287-333. 
Broom, D. C., Jutkiewicz, E. M., Folk, J. E., Traynor, J. R., Rice, K. C., \& Woods, J. H. 2002. Nonpeptidic $\delta$-opioid receptor agonists reduce immobility in the forced swim assay in rats. Neuropsychopharmacology, 26(6), 744-755.

Bruchas, M., Land, B., \& Chavkin, C. 2010. The dynorphin/kappa opioid system as a modulator of stress-induced and pro-addictive behaviors. Brain research, 1314, 44-55.

Bruchas, M. R., \& Chavkin, C. 2010. Kinase cascades and ligand-directed signaling at the kappa opioid receptor. Psychopharmacology (Berl), 210(2), 137-147. doi:10.1007/s00213-0101806-y

Bruchas, M. R., Land, B. B., Aita, M., Xu, M., Barot, S. K., Li, S., \& Chavkin, C. 2007. Stress-induced p38 mitogen-activated protein kinase activation mediates kappa-opioid-dependent dysphoria. J Neurosci, 27(43), 11614-11623. doi:10.1523/JNEUROSCI.3769-07.2007

Bruchas, M. R., Land, B. B., Lemos, J. C., \& Chavkin, C. 2009. CRF1-R activation of the dynorphin/kappa opioid system in the mouse basolateral amygdala mediates anxiety-like behavior. PloS one, 4(12), e8528.

Bruchas, M. R., \& Roth, B. L. 2016. New technologies for elucidating opioid receptor function. Trends in pharmacological sciences, 37(4), 279-289.

Bruchas, M. R., Schindler, A. G., Shankar, H., Messinger, D. I., Miyatake, M., Land, B. B., . . . Quintana, A. 2011. Selective p38 $\alpha$ MAPK deletion in serotonergic neurons produces stress resilience in models of depression and addiction. Neuron, 71(3), 498-511.

Brust, T. F., Morgenweck, J., Kim, S. A., Rose, J. H., Locke, J. L., Schmid, C. L., . . Scarry, S. M. 2016. Biased agonists of the kappa opioid receptor suppress pain and itch without causing sedation or dysphoria. Sci. Signal., 9(456), ra117-ra117.

Buchel, C., Miedl, S., \& Sprenger, C. 2018. Hedonic processing in humans is mediated by an opioidergic mechanism in a mesocorticolimbic system. eLife, 7, e39648.

Bunney, B., \& Aghajanian, G. 1978. d-Amphetamine-induced depression of central dopamine neurons: evidence for mediation by both autoreceptors and a striato-nigral feedback pathway. Naunyn-Schmiedeberg's archives of pharmacology, 304(3), 255-261.

Burnette, W., Bailey, M. D., Kukoyi, S., Blakely, R., Trowbridge, C., \& Justice, J. 1996. Human norepinephrine transporter kinetics using rotating disk electrode voltammetry. Analytical chemistry, 68(17), 2932-2938.

Butelman, E. R., \& Kreek, M.-J. 2001. k-Opioid receptor agonist-induced prolactin release in primates is blocked by dopamine D2-like receptor agonists. European journal of pharmacology, 423(2-3), 243-249.

Butelman, E. R., Prisinzano, T. E., Deng, H., Rus, S., \& Kreek, M. J. 2009. Unconditioned behavioral effects of the powerful k-opioid hallucinogen salvinorin $A$ in nonhuman primates: fast onset and entry into cerebrospinal fluid. Journal of Pharmacology and Experimental Therapeutics, 328(2), 588-597.

Butelman, E. R., Yuferov, V., \& Kreek, M. J. 2012. k-opioid receptor/dynorphin system: genetic and pharmacotherapeutic implications for addiction. Trends in neurosciences, 35(10), 587596.

Cahill, C. M., Holdridge, S. V., \& Morinville, A. 2007. Trafficking of $\delta$-opioid receptors and other Gprotein-coupled receptors: implications for pain and analgesia. Trends in pharmacological sciences, 28(1), 23-31.

Cahill, C. M., Morinville, A., Lee, M.-C., Vincent, J.-P., Collier, B., \& Beaudet, A. 2001. Prolonged morphine treatment targets $\delta$ opioid receptors to neuronal plasma membranes and enhances $\delta$-mediated antinociception. Journal of Neuroscience, 21(19), 7598-7607.

Carboni, E., Tanda, G., Frau, R., \& Chiara, G. D. 1990. Blockade of the noradrenaline carrier increases extracellular dopamine concentrations in the prefrontal cortex: evidence that 
dopamine is taken up in vivo by noradrenergic terminals. Journal of neurochemistry, 55(3), 1067-1070.

Carelli, R. M., \& ljames, S. G. 2000. Nucleus accumbens cell firing during maintenance, extinction, and reinstatement of cocaine self-administration behavior in rats. Brain research, 866(12), 44-54.

Carlezon, W. A., Béguin, C., DiNieri, J. A., Baumann, M. H., Richards, M. R., Todtenkopf, M. S., . . . Cohen, B. M. 2006. Depressive-like effects of the k-opioid receptor agonist salvinorin A on behavior and neurochemistry in rats. Journal of Pharmacology and Experimental Therapeutics, 316(1), 440-447.

Carlezon, W. A., \& Wise, R. A. 1996. Rewarding actions of phencyclidine and related drugs in nucleus accumbens shell and frontal cortex. Journal of neuroscience, 16(9), 3112-3122.

Carr, G. V., Bangasser, D. A., Bethea, T., Young, M., Valentino, R. J., \& Lucki, I. 2010. Antidepressant-like effects of k-opioid receptor antagonists in Wistar Kyoto rats. Neuropsychopharmacology, 35(3), 752.

Carr, G. V., \& Lucki, I. 2010. Comparison of the kappa-opioid receptor antagonist DIPPA in tests of anxiety-like behavior between Wistar Kyoto and Sprague Dawley rats. Psychopharmacology, 210(2), 295-302.

Carr, G. V., \& Mague, S. D. 2008. p38: The Link between the k-Opioid Receptor and Dysphoria. Journal of Neuroscience, 28(10), 2299-2300.

Carroll, M. E. 1998. Acquisition and reacquisition (relapse) of drug abuse: modulation by alternative reinforcers. NIDA Res Monogr, 169, 6-25.

Carter, L. P., \& Griffiths, R. R. 2009. Principles of laboratory assessment of drug abuse liability and implications for clinical development. Drug and Alcohol dependence, 105, S14-S25.

Casey, K. L., Minoshima, S., Morrow, T. J., \& Koeppe, R. A. (1996). Comparison of human cerebral activation pattern during cutaneous warmth, heat pain, and deep cold pain (Vol. 76).

Castagné, V., Moser, P., Roux, S., \& Porsolt, R. D. 2010. Rodent models of depression: forced swim and tail suspension behavioral despair tests in rats and mice. Current Protocols in Pharmacology, 49(1), 5.8. 1-5.8. 14.

Castro, D. C., \& Berridge, K. C. 2014. Opioid hedonic hotspot in nucleus accumbens shell: mu, delta, and kappa maps for enhancement of sweetness "liking" and "wanting". Journal of Neuroscience, 34(12), 4239-4250.

Cavaletti, G., Cavalletti, E., Oggioni, N., Sottani, C., Minoia, C., D'incalci, M., . . Tredici, G. 2000. Distribution of paclitaxel within the nervous system of the rat after repeated intravenous administration. Neurotoxicology, 21(3), 389-393.

Center, M. P. 2011. Pharmacology of opioids in the treatment of chronic pain syndromes. Pain physician, 14, E343-E360.

Chang, H. M., Berde, C. B., Holz, G. G. t., Steward, G. F., \& Kream, R. M. (1989). Sufentanil, morphine, met-enkephalin, and kappa-agonist $(\mathrm{U}-50,488 \mathrm{H})$ inhibit substance $P$ release from primary sensory neurons: a model for presynaptic spinal opioid actions. Anesthesiology, 70(4), 672-677.

Chartoff, E., Sawyer, A., Rachlin, A., Potter, D., Pliakas, A., \& Carlezon, W. A. 2012. Blockade of kappa opioid receptors attenuates the development of depressive-like behaviors induced by cocaine withdrawal in rats. Neuropharmacology, 62(1), 167-176.

Chartoff, E. H., Potter, D., Damez-Werno, D., Cohen, B. M., \& Carlezon, W. A. 2008. Exposure to the selective $\mathrm{k}$-opioid receptor agonist salvinorin A modulates the behavioral and molecular effects of cocaine in rats. Neuropsychopharmacology, 33(11), 2676-2687.

Chavkin, C. 2011. The therapeutic potential of $\mathrm{k}$-opioids for treatment of pain and addiction. Neuropsychopharmacology, 36(1), 369. 
Chavkin, C., James, I. F., \& Goldstein, A. 1982. Dynorphin is a specific endogenous ligand of the kappa opioid receptor. Science, 215(4531), 413-415.

Chavkin, C., Schattauer, S. S., \& Levin, J. R. (2014). Arrestin-mediated activation of p38 MAPK: molecular mechanisms and behavioral consequences Arrestins-Pharmacology and Therapeutic Potential (pp. 281-292): Springer.

Che, T., Majumdar, S., Zaidi, S. A., Ondachi, P., McCorvy, J. D., Wang, S., . . Krumm, B. E. 2018. Structure of the nanobody-stabilized active state of the kappa opioid receptor. Cell, 172(12), 55-67. e15.

Cheatle, M., Comer, D., Wunsch, M., Skoufalos, A., \& Reddy, Y. 2014. Treating pain in addicted patients: recommendations from an expert panel. Population health management, 17(2), 79-89.

Chefer, V., Moron, J., Hope, B., Rea, W., \& Shippenberg, T. 2000. Kappa-opioid receptor activation prevents alterations in mesocortical dopamine neurotransmission that occur during abstinence from cocaine. Neuroscience, 101(3), 619-627.

Chefer, V., \& Shippenberg, T. 2009. Augmentation of morphine-induced sensitization but reduction in morphine tolerance and reward in delta-opioid receptor knockout mice. Neuropsychopharmacology, 34(4), 887-898.

Chefer, V., Thompson, A. C., \& Shippenberg, T. S. 1999. Modulation of Cocaine-induced Sensitization by k-Opioid Receptor Agonists: Role of the Nucleus Accumbens and Medial Prefrontal Cortex. Annals of the New York Academy of Sciences, 877(1), 803-806.

Chefer, V. I., Bäckman, C. M., Gigante, E. D., \& Shippenberg, T. S. 2013. Kappa opioid receptors on dopaminergic neurons are necessary for kappa-mediated place aversion. Neuropsychopharmacology, 38(13), 2623.

Chen, N., Trowbridge, C., \& Justice Jr, J. 1998. Voltammetric studies on mechanisms of dopamine efflux in the presence of substrates and cocaine from cells expressing human norepinephrine transporter. Journal of neurochemistry, 71(2), 653-665.

Chen, N., Trowbridge, C. G., \& Justice, J. B. 1999. Cationic modulation of human dopamine transporter: dopamine uptake and inhibition of uptake. Journal of Pharmacology and Experimental Therapeutics, 290(3), 940-949.

Chen, R., Furman, C. A., \& Gnegy, M. E. 2010. Dopamine transporter trafficking: rapid response on demand. Future neurology, 5(1), 123-134.

Chiu, Y.-T., Chen, C., Yu, D., Schulz, S., \& Liu-Chen, L.-Y. 2017. Agonist-dependent and-independent kappa opioid receptor phosphorylation showed distinct phosphorylation patterns and resulted in different cellular outcomes. Molecular pharmacology, mol. 117.108555.

Chouchkov, C. (2012). Cutaneous receptors (Vol. 54): Springer Science \& Business Media.

Chu, L. F., Clark, D. J., \& Angst, M. S. 2006. Opioid tolerance and hyperalgesia in chronic pain patients after one month of oral morphine therapy: a preliminary prospective study. The Journal of Pain, 7(1), 43-48.

Cicero, T. J., Ellis, M. S., Surratt, H. L., \& Kurtz, S. P. 2014. The changing face of heroin use in the United States: a retrospective analysis of the past 50 years. JAMA psychiatry, 71(7), 821826.

Ciliax, B. J., Drash, G. W., Staley, J. K., Haber, S., Mobley, C. J., Miller, G. W., . . Levey, A. I. 1999. Immunocytochemical localization of the dopamine transporter in human brain. Journal of Comparative Neurology, 409(1), 38-56.

Ciliax, B. J., Heilman, C., Demchyshyn, L. L., Pristupa, Z. B., Ince, E., Hersch, S. M., . . Levey, A. I. 1995. The dopamine transporter: immunochemical characterization and localization in brain. The Journal of neuroscience, 15(3), 1714-1723.

Clayton, J. A., \& Collins, F. S. 2014. Policy: NIH to balance sex in cell and animal studies. Nature News, 509(7500), 282. 
Claus, E. D., Ewing, S. W. F., Filbey, F. M., Sabbineni, A., \& Hutchison, K. E. 2011. Identifying neurobiological phenotypes associated with alcohol use disorder severity. Neuropsychopharmacology, 36(10), 2086.

Clow, D. W., \& Jhamandas, K. 1988. Effects of a K-receptor agonist, U-50,488 H, on the release of endogenous brain dopamine. Canadian journal of physiology and pharmacology, 66(1), 128-133.

Codd, E. E., Carson, J. R., Colburn, R. W., Stone, D. J., Van Besien, C. R., Zhang, S.-P., . . Molino, L. 2009. JNJ-20788560 [9-(8-azabicyclo [3.2. 1] oct-3-ylidene)-9H-xanthene-3-carboxylic acid diethylamide], a selective delta opioid receptor agonist, is a potent and efficacious antihyperalgesic agent that does not produce respiratory depression, pharmacologic tolerance, or physical dependence. Journal of Pharmacology and Experimental Therapeutics, 329(1), 241-251.

Collins, S., Gerdes, R., D'addario, C., \& Izenwasser, S. 2001a. Kappa opioid agonists alter dopamine markers and cocaine-stimulated locomotor activity. Behavioural pharmacology, 12(4), 237-245.

Collins, S. L., D'Addario, C., \& Izenwasser, S. 2001b. Effects of k-opioid receptor agonists on longterm cocaine use and dopamine neurotransmission. European journal of pharmacology, 426(1-2), 25-34.

Combe, R., Bramwell, S., \& Field, M. J. 2004. The monosodium iodoacetate model of osteoarthritis: a model of chronic nociceptive pain in rats? Neuroscience letters, 370(2-3), 236-240.

Comer, S. D., Hoenicke, E., Sable, A., McNutt, R., Chang, K., De Costa, B., . . . Woods, J. 1993. Convulsive effects of systemic administration of the delta opioid agonist BW373U86 in mice. Journal of Pharmacology and Experimental Therapeutics, 267(2), 888-895.

Commons, K. G., Cholanians, A. B., Babb, J. A., \& Ehlinger, D. G. 2017. The rodent forced swim test measures stress-coping strategy, not depression-like behavior. ACS chemical neuroscience, 8(5), 955-960.

Compton, W. M., \& Volkow, N. D. 2006. Major increases in opioid analgesic abuse in the United States: concerns and strategies. Drug and alcohol dependence, 81(2), 103-107.

Cools, R., Barker, R. A., Sahakian, B. J., \& Robbins, T. W. 2001. Mechanisms of cognitive set flexibility in Parkinson's disease. Brain, 124(12), 2503-2512.

Corder, G., Castro, D. C., Bruchas, M. R., \& Scherrer, G. 2018. Endogenous and exogenous opioids in pain. Annual review of neuroscience(0).

Cox, S. 2016. Annual Update of Key Results 2015/16: New Zealand Health Survey. Wellington: Ministry of Health.

Crawford, C., McDougall, S., Bolanos, C., Hall, S., \& Berger, S. 1995. The effects of the kappa agonist U-50,488 on cocaine-induced conditioned and unconditioned behaviors and Fos immunoreactivity. Psychopharmacology, 120(4), 392-399.

Crowley, R. S., Riley, A. P., Sherwood, A. M., Groer, C. E., Shivaperumal, N., Biscaia, M., . . Kivell, B. M. 2016. Synthetic studies of neoclerodane diterpenes from Salvia divinorum: identification of a potent and centrally acting $\mu$ opioid analgesic with reduced abuse liability. Journal of medicinal chemistry, 59(24), 11027-11038.

Culverhouse, A. 2016. Exploring the aversive and anxiogenic effects of novel kappa opioid receptor agonists in rats.

Dahan, A., Sarton, E., Teppema, L., Olievier, C., Nieuwenhuijs, D., Matthes, H. W., \& Kieffer, B. L. 2001. Anesthetic potency and influence of morphine and sevoflurane on respiration in $\mu$ opioid receptor knockout mice. Anesthesiology: The Journal of the American Society of Anesthesiologists, 94(5), 824-832. 
Dahlhamer, J., Lucas, J., Zelaya, C., Nahin, R., Mackey, S., DeBar, L., . . . Helmick, C. 2018. Prevalence of chronic pain and high-impact chronic pain among adults-United States, 2016. Morbidity and Mortality Weekly Report, 67(36), 1001.

Daniels, D. J., Kulkarni, A., Xie, Z., Bhushan, R. G., \& Portoghese, P. S. 2005. A bivalent ligand (KDAN-18) containing $\delta$-antagonist and $\mathrm{k}$-agonist pharmacophores bridges $\delta 2$ and $\mathrm{k} 1$ opioid receptor phenotypes. Journal of medicinal chemistry, 48(6), 1713-1716.

Darcq, E., \& Kieffer, B. L. 2018. Opioid receptors: drivers to addiction? Nature Reviews Neuroscience, 1.

Daws, L. C., Callaghan, P. D., Morón, J. A., Kahlig, K. M., Shippenberg, T. S., Javitch, J. A., \& Galli, A. 2002. Cocaine increases dopamine uptake and cell surface expression of dopamine transporters. Biochemical and biophysical research communications, 290(5), 1545-1550.

Dayan, P., \& Balleine, B. W. 2002. Reward, motivation, and reinforcement learning. Neuron, 36(2), 285-298.

De Kloet, E., \& Molendijk, M. 2016. Coping with the forced swim stressor: towards understanding an adaptive mechanism. Neural plasticity, 2016.

De Ruddere, L., \& Craig, K. D. 2016. Understanding stigma and chronic pain: a-state-of-the-art review. Pain, 157(8), 1607-1610.

de Vries, T. J., Babovic-Vuksanovic, D., Elmer, G., \& Shippenberg, T. S. 1995. Lack of involvement of $\delta$-opioid receptors in mediating the rewarding effects of cocaine. Psychopharmacology, 120(4), 442-448. doi:10.1007/bf02245816

De Wit, H. 1996. Priming effects with drugs and other reinforcers. Experimental and Clinical Psychopharmacology, 4(1), 5.

De Wit, H., \& Stewart, J. 1981. Reinstatement of cocaine-reinforced responding in the rat. Psychopharmacology, 75(2), 134-143.

Deneau, G., \& Inoki, R. 1967. Nicotine self-administration in monkeys. Annals of the New York Academy of Sciences, 142(1), 277-279.

Deneau, G., Yanagita, T., \& Seevers, M. 1969. Self-administration of psychoactive substances by the monkey. Psychopharmacologia, 16(1), 30-48.

Deng, L., Guindon, J., Cornett, B. L., Makriyannis, A., Mackie, K., \& Hohmann, A. G. 2015. Chronic cannabinoid receptor 2 activation reverses paclitaxel neuropathy without tolerance or cannabinoid receptor 1-dependent withdrawal. Biological psychiatry, 77(5), 475-487.

Deroche-Gamonet, V., Belin, D., \& Piazza, P. V. 2004. Evidence for addiction-like behavior in the rat. Science, 305(5686), 1014-1017.

Di Chiara, G. 1998. A motivational learning hypothesis of the role of mesolimbic dopamine in compulsive drug use. Journal of psychopharmacology, 12(1), 54-67.

Di Chiara, G., \& Imperato, A. 1988a. Drugs abused by humans preferentially increase synaptic dopamine concentrations in the mesolimbic system of freely moving rats. Proceedings of the National Academy of Sciences, 85(14), 5274-5278.

Di Chiara, G., \& Imperato, A. 1988b. Opposite effects of mu and kappa opiate agonists on dopamine release in the nucleus accumbens and in the dorsal caudate of freely moving rats. Journal of Pharmacology and Experimental Therapeutics, 244(3), 1067-1080.

Di Ciano, P., Robbins, T. W., \& Everitt, B. J. 2008. Differential effects of nucleus accumbens core, shell, or dorsal striatal inactivations on the persistence, reacquisition, or reinstatement of responding for a drug-paired conditioned reinforcer. Neuropsychopharmacology, 33(6), 1413.

Dietis, N. (2012). Strategies to reduce morphine tolerance in cancer: evaluation of the bifunctional opioid UFP-505. University of Leicester. 
Dietis, N., Guerrini, R., Calo, G., Salvadori, S., Rowbotham, D., \& Lambert, D. 2009. Simultaneous targeting of multiple opioid receptors: a strategy to improve side-effect profile. British journal of anaesthesia, 103(1), 38-49.

Dikshtein, Y., Barnea, R., Kronfeld, N., Lax, E., Roth-Deri, I., Friedman, A., . . Ben-Tzion, M. 2013. $\beta$-endorphin via the delta opioid receptor is a major factor in the incubation of cocaine craving. Neuropsychopharmacology, 38(12), 2508.

DiMattio, K. M., Ehlert, F. J., \& Liu-Chen, L.-Y. 2015. Intrinsic relative activities of $\mathrm{K}$ opioid agonists in activating $\mathrm{G} \alpha$ proteins and internalizing receptor: Differences between human and mouse receptors. European journal of pharmacology, 761, 235-244.

Ding, H., Czoty, P. W., Kiguchi, N., Cami-Kobeci, G., Sukhtankar, D. D., Nader, M. A., . . Ko, M.-C. 2016. A novel orvinol analog, BU08028, as a safe opioid analgesic without abuse liability in primates. Proceedings of the National Academy of Sciences, 113(37), E5511-E5518.

DiNieri, J. A., Nemeth, C. L., Parsegian, A., Carle, T., Gurevich, V. V., Gurevich, E., . . Carlezon, W. A. 2009. Altered sensitivity to rewarding and aversive drugs in mice with inducible disruption of cAMP response element-binding protein function within the nucleus accumbens. Journal of Neuroscience, 29(6), 1855-1859.

Do Carmo, G. P., Folk, J. E., Rice, K. C., Chartoff, E., Carlezon Jr, W. A., \& Negus, S. S. 2009. The selective non-peptidic delta opioid agonist SNC80 does not facilitate intracranial selfstimulation in rats. European journal of pharmacology, 604(1-3), 58-65.

Dobrin, C. V., \& Roberts, D. C. (2012). Cocaine self-administration in rats: discrete trials procedures Psychiatric Disorders (pp. 291-302): Springer.

Dominick, C., Blyth, F., \& Nicholas, M. 2011. Patterns of chronic pain in the New Zealand population. NZ Med J, 124(1337), 63-76.

Dougherty, P. M., Cata, J. P., Cordella, J. V., Burton, A., \& Weng, H.-R. 2004. Taxol-induced sensory disturbance is characterized by preferential impairment of myelinated fiber function in cancer patients. Pain, 109(1-2), 132-142.

Du, C., Duan, Y., Wei, W., Cai, Y., Chai, H., Lv, J., . . Xie, X. 2016. Kappa opioid receptor activation alleviates experimental autoimmune encephalomyelitis and promotes oligodendrocytemediated remyelination. Nature communications, 7, 11120.

Dubin, A. E., \& Patapoutian, A. 2010. Nociceptors: the sensors of the pain pathway. The Journal of clinical investigation, 120(11), 3760-3772.

Dueñas, M., Salazar, A., Ojeda, B., Fernández-Palacín, F., Micó, J. A., Torres, L. M., \& Failde, I. 2015. A nationwide study of chronic pain prevalence in the general Spanish population: identifying clinical subgroups through cluster analysis. Pain Medicine, 16(4), 811-822.

Dunham, N., \& Miya, T. 1957. A note on a simple apparatus for detecting neurological deficit in rats and mice. Journal of the American Pharmaceutical Association, 46(3), 208-209.

Dykstra, L. A., \& Woods, J. H. 1986. A tail withdrawal procedure for assessing analgesic activity in rhesus monkeys.

Earles, C., \& Schenk, J. O. 1998. Rotating disk electrode voltammetric measurements of dopamine transporter activity: an analytical evaluation. Analytical biochemistry, 264(2), 191-198.

Ebner, S. R., Roitman, M. F., Potter, D. N., Rachlin, A. B., \& Chartoff, E. H. 2010. Depressive-like effects of the kappa opioid receptor agonist salvinorin $A$ are associated with decreased phasic dopamine release in the nucleus accumbens. Psychopharmacology, 210(2), 241252.

Eddy, N. B., \& Leimbach, D. 1953. Synthetic analgesics. II. Dithienylbutenyl-and dithienylbutylamines. Journal of Pharmacology and Experimental Therapeutics, 107(3), 385-393. 
Ehrich, J. M., Messinger, D. I., Knakal, C. R., Kuhar, J. R., Schattauer, S. S., Bruchas, M. R., . . . Chavkin, C. 2015. Kappa opioid receptor-induced aversion requires p38 MAPK activation in VTA dopamine neurons. Journal of Neuroscience, 35(37), 12917-12931.

Elsworth, J. D., \& Roth, R. H. 1997. Dopamine synthesis, uptake, metabolism, and receptors: relevance to gene therapy of Parkinson's disease. Experimental neurology, 144(1), 4-9.

Endoh, T., Matsuura, H., Tanaka, C., \& Nagase, H. 1992. Nor-binaltorphimine: a potent and selective kappa-opioid receptor antagonist with long-lasting activity in vivo. Archives internationales de pharmacodynamie et de therapie, 316, 30-42.

Epstein, D. H., Preston, K. L., Stewart, J., \& Shaham, Y. 2006. Toward a model of drug relapse: an assessment of the validity of the reinstatement procedure. Psychopharmacology, 189(1), $1-16$.

Eriksen, J., Bjorn-Yoshimoto, W. E., Jorgensen, T. N., Newman, A. H., \& Gether, U. 2010. Postendocytic sorting of constitutively internalized dopamine transporter in cell lines and dopaminergic neurons. Journal of Biological Chemistry, jbc. M110. 131003.

Eriksson, P., Nilsson, M., Wågberg, M., Hansson, E., \& Rönnbäck, L. 1993. Kappa-opioid receptors on astrocytes stimulate L-type Ca2+ channels. Neuroscience, 54(2), 401-407.

Everitt, B. J., \& Robbins, T. W. 2000. Second-order schedules of drug reinforcement in rats and monkeys: measurement of reinforcing efficacy and drug-seeking behaviour. Psychopharmacology, 153(1), 17-30.

Everitt, B. J., \& Robbins, T. W. 2005. Neural systems of reinforcement for drug addiction: from actions to habits to compulsion. Nature neuroscience, 8(11), 1481.

Ewald, A. (2016). Investigating the Anti-cocaine Effects of Novel Kappa Opioid Receptor Agonists: Behavioural and Cellular Actions of Salvinorin A Analogues: a Thesis Submitted to the Victoria University of Wellington in Fulfilment of the Requirements for the Degree of Doctor of Philosophy. Victoria University of Wellington.

Ewald, A. W., Bosch, P. J., Culverhouse, A., Crowley, R. S., Neuenswander, B., Prisinzano, T. E., \& Kivell, B. M. 2017. The $C-2$ derivatives of salvinorin $A$, ethoxymethyl ether Sal $B$ and $\beta$ tetrahydropyran Sal $B$, have anti-cocaine properties with minimal side effects. Psychopharmacology, 234(16), 2499-2514.

Fairbanks, C. A. 2003. Spinal delivery of analgesics in experimental models of pain and analgesia. Advanced drug delivery reviews, 55(8), 1007-1041.

Fantegrossi, W. E., Kugle, K. M., Valdes III, L. J., Koreeda, M., \& Woods, J. H. 2005. Kappa-opioid receptor-mediated effects of the plant-derived hallucinogen, salvinorin $A$, on inverted screen performance in the mouse. Behavioural pharmacology, 16(8), 627-633.

Faure, A., Haberland, U., Condé, F., \& El Massioui, N. 2005. Lesion to the nigrostriatal dopamine system disrupts stimulus-response habit formation. Journal of Neuroscience, 25(11), 2771-2780.

Fayaz, A., Croft, P., Langford, R., Donaldson, L., \& Jones, G. 2016. Prevalence of chronic pain in the UK: a systematic review and meta-analysis of population studies. BMJ open, 6(6), e010364.

Feng, Y., He, X., Yang, Y., Chao, D., H Lazarus, L., \& Xia, Y. 2012. Current research on opioid receptor function. Current drug targets, 13(2), 230-246.

Ferster, C. B., \& Skinner, B. F. 1957. Schedules of reinforcement.

Fibiger, H. C., \& Phillips, A. 1988. Mesocorticolimbic dopamine systems and reward. Annals of the New York Academy of Sciences, 537(1), 206-215.

File, S. E., Lippa, A. S., Beer, B., \& Lippa, M. T. 2004. Animal tests of anxiety. Current protocols in neuroscience, 26(1), 8.3. 1-8.3. 22. 
Filliol, D., Ghozland, S., Chluba, J., Martin, M., Matthes, H. W., Simonin, F., . . LeMeur, M. 2000. Mice deficient for $\delta$-and $\mu$-opioid receptors exhibit opposing alterations of emotional responses. Nature genetics, 25(2), 195.

Fink, J. S., \& Smith, G. P. 1979. Decreased locomotor and investigatory exploration after denervation of catecholamine terminal fields in the forebrain of rats. Journal of Comparative and Physiological Psychology, 93(1), 34.

Finnerup, N. B., Attal, N., Haroutounian, S., McNicol, E., Baron, R., Dworkin, R. H., . . Jensen, T. S. 2015. Pharmacotherapy for neuropathic pain in adults: a systematic review and metaanalysis. The Lancet Neurology, 14(2), 162-173.

Flatters, S., Dougherty, P. M., \& Colvin, L. 2017. Clinical and preclinical perspectives on chemotherapy-induced peripheral neuropathy (CIPN): a narrative review. BJA: British Journal of Anaesthesia, 119(4), 737-749.

Freed, C., Revay, R., Vaughan, R. A., Kriek, E., Grant, S., Uhl, G. R., \& Kuhar, M. J. 1995. Dopamine transporter immunoreactivity in rat brain. Journal of Comparative Neurology, 359(2), 340349.

Freeman, K. B., Naylor, J. E., Prisinzano, T. E., \& Woolverton, W. L. 2014. Assessment of the kappa opioid agonist, salvinorin $A$, as a punisher of drug self-administration in monkeys. Psychopharmacology, 231(14), 2751-2758.

Gainetdinov, R. R., Wetsel, W. C., Jones, S. R., Levin, E. D., Jaber, M., \& Caron, M. G. 1999. Role of serotonin in the paradoxical calming effect of psychostimulants on hyperactivity. science, 283(5400), 397-401.

Gal, T. J., DiFazio, C. A., \& Moscicki, J. 1982. Analgesic and respiratory depressant activity of nalbuphine: a comparison with morphine. Anesthesiology, 57(5), 367-374.

Gallantine, E. L., \& Meert, T. F. 2008. Antinociceptive and Adverse Effects of $\mu$-and k-Opioid Receptor Agonists: A Comparison of Morphine and U50488-H. Basic \& clinical pharmacology \& toxicology, 103(5), 419-427.

Gardner, E. L. 1997. Brain reward mechanisms. Substance abuse: A comprehensive textbook, 5185.

Gaskin, D. J., \& Richard, P. 2012. The economic costs of pain in the United States. The Journal of Pain, 13(8), 715-724.

Gavériaux-Ruff, C., \& Kieffer, B. L. 2011. Delta opioid receptor analgesia: recent contributions from pharmacology and molecular approaches. Behavioural pharmacology, 22(5-6), 405.

Gavériaux-Ruff, C., Karchewski, L. A., Hever, X., Matifas, A., \& Kieffer, B. L. 2008. Inflammatory pain is enhanced in delta opioid receptor-knockout mice. European Journal of Neuroscience, 27(10), 2558-2567.

Gear, R. W., Gordon, N. C., Heller, P. H., Paul, S., Miaskowski, C., \& Levine, J. D. 1996. Gender difference in analgesic response to the kappa-opioid pentazocine. Neuroscience letters, 205(3), 207-209.

Gillett, K., Harshberger, E., \& Valdez, G. R. 2013. Protracted withdrawal from ethanol and enhanced responsiveness stress: regulation via the dynorphin/kappa opioid receptor system. Alcohol, 47(5), 359-365.

Glick, S. D., Maisonneuve, I. M., Raucci, J., \& Sydney, A. 1995. Kappa opioid inhibition of morphine and cocaine self-administration in rats. Brain Research, 681(1-2), 147-152. doi:http://dx.doi.org/10.1016/0006-8993(95)00306-B

Glick, S. D., Visker, K. E., \& Maisonneuve, I. M. 1998. Effects of cyclazocine on cocaine selfadministration in rats. European journal of pharmacology, 357(1), 9-14.

Global Drug Survey, G. (2018). Last 12 month prevalence of top 20 drugs In: Winstock, D. A. (ed.). 
Göktalay, G., Cavun, S., Levendusky, M. C., Hamilton, J. R., \& Millington, W. R. 2006. Glycylglutamine inhibits nicotine conditioned place preference and withdrawal. European journal of pharmacology, 530(1-2), 95-102.

Goodman, A. J., Le Bourdonnec, B., \& Dolle, R. E. 2007. Mu opioid receptor antagonists: recent developments. ChemMedChem, 2(11), 1552-1570.

Gramsch, C., Höllt, V., Pasi, A., Mehraein, P., \& Herz, A. 1982. Immunoreactive dynorphin in human brain and pituitary. Brain Research, 233(1), 65-74.

Grant, B. F. 1996. Prevalence and correlates of drug use and DSM-IV drug dependence in the United States: results of the National Longitudinal Alcohol Epidemiologic Survey. Journal of substance abuse, 8(2), 195-210.

Grant, B. F., Chou, S. P., Saha, T. D., Pickering, R. P., Kerridge, B. T., Ruan, W. J., . . . Fan, A. 2017. Prevalence of 12-month alcohol use, high-risk drinking, and DSM-IV alcohol use disorder in the United States, 2001-2002 to 2012-2013: results from the National Epidemiologic Survey on Alcohol and Related Conditions. JAMA psychiatry, 74(9), 911-923.

Gray, A. M., Rawls, S. M., Shippenberg, T. S., \& McGinty, J. F. 1999. The K-Opioid Agonist, U-69593, Decreases Acute Amphetamine-Evoked Behaviors and Calcium-Dependent Dialysate Levels of Dopamine and Glutamate in the Ventral Striatum. Journal of neurochemistry, 73(3), 1066-1074.

Greedy, B. M., Bradbury, F., Thomas, M. P., Grivas, K., Cami-Kobeci, G., Archambeau, A., . . Lewis, J. W. 2013. Orvinols with mixed kappa/mu opioid receptor agonist activity. Journal of medicinal chemistry, 56(8), 3207-3216.

Grospe, G. M., Baker, P. M., \& Ragozzino, M. E. 2018. Cognitive flexibility deficits following 6OHDA lesions of the rat dorsomedial striatum. Neuroscience, 374, 80-90.

Grudt, T. J., \& Williams, J. T. 1993. kappa-Opioid receptors also increase potassium conductance. Proceedings of the National Academy of Sciences, 90(23), 11429-11432.

Gulley, J. M., \& Zahniser, N. R. 2003. Rapid regulation of dopamine transporter function by substrates, blockers and presynaptic receptor ligands. European journal of pharmacology, 479(1-3), 139-152.

Guo, S., Zhou, D. F., Sun, H. Q., Wu, G. Y., Haile, C. N., Kosten, T. A., . . Zhang, X. Y. 2007. Association of functional catechol O-methyl transferase (COMT) Val108Met polymorphism with smoking severity and age of smoking initiation in Chinese male smokers. Psychopharmacology, 190(4), 449-456.

Gupta, A., Décaillot, F. M., \& Devi, L. A. 2006. Targeting opioid receptor heterodimers: strategies for screening and drug development. The AAPS journal, 8(1), E153-E159.

Gupta, A., Gomes, I., Bobeck, E. N., Fakira, A. K., Massaro, N. P., Sharma, I., . . Devi, L. A. 2016. Collybolide is a novel biased agonist of $\mathrm{K}$-opioid receptors with potent antipruritic activity. Proceedings of the National Academy of Sciences, 201521825.

Gureje, O., Von Korff, M., Simon, G. E., \& Gater, R. 1998. Persistent pain and well-being: a World Health Organization study in primary care. Jama, 280(2), 147-151.

Hagan, C. E., Neumaier, J. F., \& Schenk, J. O. 2010. Rotating disk electrode voltammetric measurements of serotonin transporter kinetics in synaptosomes. Journal of neuroscience methods, 193(1), 29-38.

Hall, W., Teesson, M., Lynskey, M., \& Degenhardt, L. 1999. The 12-month prevalence of substance use and ICD-10 substance use disorders in Australian adults: findings from the National Survey of Mental Health and Well-Being. Addiction, 94(10), 1541-1550.

Han, J. S., Bird, G. C., Li, W., Jones, J., \& Neugebauer, V. 2005. Computerized analysis of audible and ultrasonic vocalizations of rats as a standardized measure of pain-related behavior. Journal of neuroscience methods, 141(2), 261-269. 
Haney, M., \& Spealman, R. 2008. Controversies in translational research: drug self-administration. Psychopharmacology, 199(3), 403-419.

Harden, M. T., Smith, S. E., Niehoff, J. A., McCurdy, C. R., \& Taylor, G. T. 2012. Antidepressive effects of the $\mathrm{k}$-opioid receptor agonist salvinorin $\mathrm{A}$ in a rat model of anhedonia. Behavioural pharmacology, 23(7), 710-715.

Harshberger, E. 2010. Kappa opioid regulation of stress-related behavior. McNair Scholars Journal, 14(1), 6.

Heal, D. J., Gosden, J., \& Smith, S. L. 2014. Dopamine reuptake transporter (DAT) “inverse agonism"-a novel hypothesis to explain the enigmatic pharmacology of cocaine. Neuropharmacology, 87, 19-40.

Hedegaard, H., Miniño, A. M., \& Warner, M. 2018. Drug overdose deaths in the United States, 1999-2017:NCHS Data Brief, no 329. Hyattsville, MD: National Centre for Health Statistics.

Henderson, J. V., Harrison, C. M., Britt, H. C., Bayram, C. F., \& Miller, G. C. 2013. Prevalence, causes, severity, impact, and management of chronic pain in Australian general practice patients. Pain Medicine, 14(9), 1346-1361.

Hershman, D., Lacchetti, C., Dworkin, R., Lavoie Smith, E., Bleeker, J., Cavaletti, G., . . Lustberg, M. American Society of Clinical O (2014) Prevention and management of chemotherapyinduced peripheral neuropathy in survivors of adult cancers: American Society of Clinical Oncology clinical practice guideline. J Clin Oncol, 32(18), 1941-1967.

Heyman, J. S., Jiang, Q., Rothman, R. B., Mosberg, H. I., \& Porreca, F. 1989. Modulation of $\mu-$ mediated antinociception by $\delta$ agonists: characterization with antagonists. European journal of pharmacology, 169(1), 43-52.

Hickey, C., \& Peelen, M. V. 2015. Neural mechanisms of incentive salience in naturalistic human vision. Neuron, 85(3), 512-518.

Hiranita, T., Soto, P. L., Newman, A. H., \& Katz, J. L. 2009. Assessment of reinforcing effects of benztropine analogs and their effects on cocaine self-administration in rats: comparisons with monoamine uptake inhibitors. Journal of Pharmacology and Experimental Therapeutics, 329(2), 677-686.

Hobbs, M., Remington, B., \& Glautier, S. 2005. Dissociation of wanting and liking for alcohol in humans: a test of the incentive-sensitisation theory. Psychopharmacology, 178(4), 493499.

Hoffman, B. J., Mezey, E., \& Brownstein, M. J. 1991. Cloning of a serotonin transporter affected by antidepressants. Science, 254(5031), 579-580.

Horan, P., de COSTA, B. R., Rice, K. C., \& Porreca, F. 1991. Differential antagonism of U69, 593-and bremazocine-induced antinociception by (-)-UPHIT: evidence of kappa opioid receptor multiplicity in mice. Journal of Pharmacology and Experimental Therapeutics, 257(3), 1154-1161.

Horan, P., Tallarida, R. J., Haaseth, R. C., Matsunaga, T. O., Hruby, V. J., \& Porreca, F. 1992. Antinociceptive interactions of opioid delta receptor agonists with morphine in mice: supra-and sub-additivity. Life sciences, 50(20), 1535-1541.

Horan, P., Taylor, J., Yamamura, H. I., \& Porreca, F. 1992. Extremely long-lasting antagonistic actions of nor-binaltorphimine (nor-BNI) in the mouse tail-flick test. Journal of Pharmacology and Experimental Therapeutics, 260(3), 1237-1243.

Horan, P. J., Mattia, A., Bilsky, E. J., Weber, S., Davis, T. P., Yamamura, H. I., . . Misicka, A. 1993. Antinociceptive profile of biphalin, a dimeric enkephalin analog. Journal of Pharmacology and Experimental Therapeutics, 265(3), 1446-1454.

Horn, A. 1973. Structure-activity relations for the inhibition of catecholamine uptake into synaptosomes from noradrenaline and dopaminergic neurones in rat brain homogenates. British journal of pharmacology, 47(2), 332-338. 
Huang, P., Tunis, J., Parry, C., Tallarida, R., \& Liu-Chen, L.-Y. 2016. Synergistic antidepressant-like effects between a kappa opioid antagonist (LY2444296) and a delta opioid agonist (ADL5859) in the mouse forced swim test. European journal of pharmacology, 781, 53-59.

Hutsell, B. A., Cheng, K., Rice, K. C., Negus, S. S., \& Banks, M. L. 2016. Effects of the kappa opioid receptor antagonist nor-binaltorphimine (nor-BNI) on cocaine versus food choice and extended-access cocaine intake in rhesus monkeys. Addiction biology, 21(2), 360-373.

Irwin, S., Bennett, D. R., Hendershot, L. C., Seevers, M., \& Houde, R. W. 1951. The effects of morphine, methadone and meperidine on some reflex responses of spinal animals to nociceptive stimulation. Journal of Pharmacology and Experimental Therapeutics, 101(2), 132-143.

Ismail, N., Girard-Bériault, F., Nakanishi, S., \& Pfaus, J. G. 2009. Naloxone, but not flupenthixol, disrupts the development of conditioned ejaculatory preference in the male rat. Behavioral neuroscience, 123(5), 992.

Ismayilova, N., \& Shoaib, M. 2010. Alteration of intravenous nicotine self-administration by opioid receptor agonist and antagonists in rats. Psychopharmacology, 210(2), 211-220.

Jackson, K., McLaughlin, J., Carroll, F., \& Damaj, M. 2013. Effects of the kappa opioid receptor antagonist, norbinaltorphimine, on stress and drug-induced reinstatement of nicotineconditioned place preference in mice. Psychopharmacology, 226(4), 763-768.

Jaggi, A. S., Jain, V., \& Singh, N. 2011. Animal models of neuropathic pain. Fundamental \& clinical pharmacology, 25(1), 1-28.

Jaquins-Gerstl, A., \& Michael, A. C. 2015. A review of the effects of FSCV and microdialysis measurements on dopamine release in the surrounding tissue. Analyst, 140(11), 36963708.

Jentsch, J. D., \& Taylor, J. R. 1999. Impulsivity resulting from frontostriatal dysfunction in drug abuse: implications for the control of behavior by reward-related stimuli. Psychopharmacology, 146(4), 373-390.

Ji, R.-R., Xu, Z.-Z., \& Gao, Y.-J. 2014. Emerging targets in neuroinflammation-driven chronic pain. Nature reviews Drug discovery, 13(7), 533.

Johanson, C. E., Balster, R. L., \& Bonese, K. 1976. Self-administration of psychomotor stimulant drugs: the effects of unlimited access. Pharmacology Biochemistry and Behavior, 4(1), 4551.

Johnson, M. W., MacLean, K. A., Reissig, C. J., Prisinzano, T. E., \& Griffiths, R. R. 2011. Human psychopharmacology and dose-effects of salvinorin A, a kappa opioid agonist hallucinogen present in the plant Salvia divinorum. Drug and alcohol dependence, 115(1-2), 150-155.

Jones, S. R., Gainetdinov, R. R., Wightman, R. M., \& Caron, M. G. 1998. Mechanisms of amphetamine action revealed in mice lacking the dopamine transporter. Journal of Neuroscience, 18(6), 1979-1986.

Jones, P., Griffin, A. M., Gawell, L., Lavoie, R., Delorme, D., Roberts, E., . . . Boulet, J. 2009. N, NDiethyl-4-[(3-hydroxyphenyl)(piperidin-4-yl) amino] benzamide derivatives: The development of diaryl amino piperidines as potent $\delta$ opioid receptor agonists with in vivo anti-nociceptive activity in rodent models. Bioorganic \& medicinal chemistry letters, 19(21), 5994-5998.

Jordan, B. A., \& Devi, L. A. 1999. G-protein-coupled receptor heterodimerization modulates receptor function. Nature, 399(6737), 697.

Julius, D., \& Basbaum, A. I. 2001. Molecular mechanisms of nociception. Nature, 413(6852), 203.

Jutkiewicz, E. M. 2006. The antidepressant-like effects of delta-opioid receptor agonists. Molecular Interventions, 6(3), 162. 
Jutkiewicz, E. M., Rice, K. C., Traynor, J. R., \& Woods, J. H. 2005. Separation of the convulsions and antidepressant-like effects produced by the delta-opioid agonist SNC80 in rats. Psychopharmacology, 182(4), 588-596.

Kabli, N., \& Cahill, C. M. 2007. Anti-allodynic effects of peripheral delta opioid receptors in neuropathic pain. Pain, 127(1-2), 84-93.

Kam, A. Y., Chan, A. S., \& Wong, Y. H. 2004. K-opioid receptor signals through Src and focal adhesion kinase to stimulate c-Jun $\mathrm{N}$-terminal kinases in transfected COS-7 cells and human monocytic THP-1 cells. Journal of Pharmacology and Experimental Therapeutics, 310(1), 301-310.

Kandel, E. R., Schwartz, J. H., Jessell, T. M., Biochemistry, D. o., Jessell, M. B. T., Siegelbaum, S., \& Hudspeth, A. (2000). Principles of neural science (Vol. 4): McGraw-hill New York.

Katsuura, Y., \& Taha, S. A. 2014. Mu opioid receptor antagonism in the nucleus accumbens shell blocks consumption of a preferred sucrose solution in an anticipatory contrast paradigm. Neuroscience, 261, 144-152.

Katz, J., Liebman, J., \& Cooper, S. 1989. The neuropharmacological basis of reward.

Katz, J. L., \& Higgins, S. T. 2003. The validity of the reinstatement model of craving and relapse to drug use. Psychopharmacology, 168(1-2), 21-30.

Kebabian, J. W. 1978. Multiple classes of dopamine receptors in mammalian central nervous system: the involvement of dopamine-sensitive adenylyl cyclase. Life sciences, 23(5), 479483.

Kenakin, T. 2007. Collateral efficacy in drug discovery: taking advantage of the good (allosteric) nature of 7TM receptors. Trends in pharmacological sciences, 28(8), 407-415.

Kenakin, T., \& Christopoulos, A. 2013. Signalling bias in new drug discovery: detection, quantification and therapeutic impact. Nature reviews Drug discovery, 12(3), 205.

Kennedy, J., Roll, J. M., Schraudner, T., Murphy, S., \& McPherson, S. 2014. Prevalence of persistent pain in the US adult population: new data from the 2010 national health interview survey. The Journal of Pain, 15(10), 979-984.

Khroyan, T. V., Polgar, W. E., Orduna, J., Montenegro, J., Jiang, F., Zaveri, N. T., \& Toll, L. 2011. Differential effects of nociceptin/orphanin FQ (NOP) receptor agonists in acute versus chronic pain: studies with bifunctional NOP/ $\mu$ receptor agonists in the sciatic nerve ligation chronic pain model in mice. Journal of Pharmacology and Experimental Therapeutics, 339(2), 687-693.

Khroyan, T. V., Zaveri, N. T., Polgar, W. E., Orduna, J., Olsen, C., Jiang, F., \& Toll, L. 2007. SR 16435 [1-(1-(bicyclo [3.3. 1] nonan-9-yl) piperidin-4-yl) indolin-2-one], a novel mixed nociceptin/orphanin $\mathrm{FQ} / \mu$-opioid receptor partial agonist: analgesic and rewarding properties in mice. Journal of Pharmacology and Experimental Therapeutics, 320(2), 934943.

Kidd, B., \& Urban, L. 2001. Mechanisms of inflammatory pain. British journal of anaesthesia, 87(1), 3-11.

Kim, H. T., Uchimoto, K., Duellman, T., \& Yang, J. 2015. Automated assessment of pain in rats using a voluntarily accessed static weight-bearing test. Physiology \& behavior, 151, 139-146.

Kishioka, S., Kiguchi, N., Kobayashi, Y., Yamamoto, C., Saika, F., Wakida, N., . . Woods, J. H. 2013. Pharmacokinetic evidence for the long-lasting effect of nor-binaltorphimine, a potent kappa opioid receptor antagonist, in mice. Neuroscience letters, 552, 98-102.

Kitamura, O., Wee, S., Specio, S. E., Koob, G. F., \& Pulvirenti, L. 2006. Escalation of methamphetamine self-administration in rats: a dose-effect function. Psychopharmacology, 186(1), 48-53. 
Kivell, B., Kahlig, K., Galli, A., Javitch, J., \& Shippenberg, T. 2004. Regulation of dopamine transporter function and cell surface expression by kappa opioid receptors. Paper presented at the Soc Neurosci Abstr.

Kivell, B., Paton, K., Kumar, N., Morani, A., Culverhouse, A., Shepherd, A., . . Prisinzano, T. 2018. Kappa Opioid Receptor Agonist Mesyl Sal B Attenuates Behavioral Sensitization to Cocaine with Fewer Aversive Side-Effects than Salvinorin A in Rodents. Molecules, 23(10), 2602.

Kivell, B., Uzelac, Z., Sundaramurthy, S., Rajamanickam, J., Ewald, A., Chefer, V., . . . Annamalai, B. 2014a. Salvinorin A regulates dopamine transporter function via a kappa opioid receptor and ERK1/2-dependent mechanism. Neuropharmacology, 86, 228-240.

Kivell, B. M., Ewald, A. W., \& Prisinzano, T. E. 2014b. Salvinorin A analogs and other kappa-opioid receptor compounds as treatments for cocaine abuse Advances in Pharmacology (Vol. 69, pp. 481-511): Elsevier.

Knoll, A. T., \& Carlezon Jr, W. A. 2010. Dynorphin, stress, and depression. Brain research, 1314, 56-73.

Knoll, A. T., Meloni, E. G., Thomas, J. B., Carroll, F. I., \& Carlezon, W. A. 2007. Anxiolytic-like effects of $\mathrm{k}$-opioid receptor antagonists in models of unlearned and learned fear in rats. Journal of Pharmacology and Experimental Therapeutics, 323(3), 838-845.

Koob, G., \& Volkow, N. (2010). Neurocircuitry of addiction. Neuropsychopharmacology: Official Publication of the American College of Neuropsychopharmacology 35, 217-38.

Koob, G. F. 2009. Neurobiological substrates for the dark side of compulsivity in addiction. Neuropharmacology, 56, 18-31.

Koob, G. F., \& Le Moal, M. 1997. Drug abuse: hedonic homeostatic dysregulation. Science, 278(5335), 52-58.

Koob, G. F., \& Le Moal, M. 2001. Drug addiction, dysregulation of reward, and allostasis. Neuropsychopharmacology, 24(2), 97.

Koob, G. F., \& Le Moal, M. 2008. Addiction and the brain antireward system. Annu. Rev. Psychol., 59, 29-53.

Kreibich, A. S., \& Blendy, J. A. 2004. cAMP response element-binding protein is required for stress but not cocaine-induced reinstatement. Journal of Neuroscience, 24(30), 6686-6692.

Kringelbach, M. L., \& Berridge, K. C. (2010). Pleasures of the brain: Series in Affective Science.

Kuehn, B. 2018. Chronic Pain Prevalence. JAMA, 320(16), 1632-1632.

Kudlowitz, D., \& Muggia, F. 2013. Defining risks of taxane neuropathy: insights from randomized clinical trials. Clinical Cancer Research, 19(17), 4570-4577.

Kühn, S., \& Gallinat, J. 2011. Common biology of craving across legal and illegal drugs-a quantitative meta-analysis of cue-reactivity brain response. European Journal of Neuroscience, 33(7), 1318-1326.

Kumagai, H., Ebata, T., Takamori, K., Muramatsu, T., Nakamoto, H., \& Suzuki, H. 2010. Effect of a novel kappa-receptor agonist, nalfurafine hydrochloride, on severe itch in 337 haemodialysis patients: a Phase III, randomized, double-blind, placebo-controlled study. Nephrol Dial Transplant, 25(4), 1251-1257. doi:10.1093/ndt/gfp588

Kuner, R. 2010. Central mechanisms of pathological pain. Nature medicine, 16(11), 1258.

Kurejova, M., Nattenmüller, U., Hildebrandt, U., Selvaraj, D., Stösser, S., \& Kuner, R. 2010. An improved behavioural assay demonstrates that ultrasound vocalizations constitute a reliable indicator of chronic cancer pain and neuropathic pain. Molecular pain, 6(1), 18.

Kuzmin, A. V., Gerrits, M. A., \& Van Ree, J. M. 1998. k-Opioid receptor blockade with norbinaltorphimine modulates cocaine self-administration in drug-naive rats. European journal of pharmacology, 358(3), 197-202. 
Kuzmin, A. V., Semenova, S., Gerrits, M. A., Zvartau, E. E., \& Van Ree, J. M. 1997. k-Opioid receptor agonist $\mathrm{U} 50,488 \mathrm{H}$ modulates cocaine and morphine self-administration in drug-naive rats and mice. European journal of pharmacology, 321(3), 265-271.

La Regina, A., Petrillo, P., Sbacchi, M., \& Tavani, A. 1988. Interaction of U-69,593 with $\mu$-, $\partial$-and kopioid binding sites and its analgesic and intestinal effects in rats. Life sciences, 42(3), 293301.

Lagard, C., Chevillard, L., Guillemyn, K., Risède, P., Laplanche, J.-L., Spetea, M., . . Mégarbane, B. 2017. Bifunctional peptide-based opioid agonist/nociceptin antagonist ligand for dual treatment of nociceptive and neuropathic pain. Pain, 158(3), 505.

Lambert, D., Bird, M., \& Rowbotham, D. (2014). Cebranopadol: a first in-class example of a nociceptin/orphanin FQ receptor and opioid receptor agonist: Oxford University Press.

Lamont, L. A., Tranquilli, W. J., \& Grimm, K. A. 2000. Physiology of pain. Veterinary Clinics: Small Animal Practice, 30(4), 703-728.

Land, B. B., Bruchas, M. R., Lemos, J. C., Xu, M., Melief, E. J., \& Chavkin, C. 2008. The dysphoric component of stress is encoded by activation of the dynorphin $\mathrm{k}$-opioid system. The Journal of Neuroscience, 28(2), 407-414.

Land, B. B., Bruchas, M. R., Schattauer, S., Giardino, W. J., Aita, M., Messinger, D., . . Chavkin, C. 2009. Activation of the kappa opioid receptor in the dorsal raphe nucleus mediates the aversive effects of stress and reinstates drug seeking. Proceedings of the National Academy of Sciences, pnas. 0910705106.

Larsen, M. B., Sonders, M. S., Mortensen, O. V., Larson, G. A., Zahniser, N. R., \& Amara, S. G. 2011. Dopamine transport by the serotonin transporter: a mechanistically distinct mode of substrate translocation. Journal of Neuroscience, 31(17), 6605-6615.

Law, P.-Y., Erickson-Herbrandson, L. J., Zha, Q. Q., Solberg, J., Chu, J., Sarre, A., \& Loh, H. H. 2005. Heterodimerization of $\mu$-and $\delta$-opioid receptors occurs at the cell surface only and requires receptor-G protein interactions. Journal of Biological Chemistry, 280(12), 1115211164.

Le Bourdonnec, B., Windh, R. T., Leister, L. K., Zhou, Q. J., Ajello, C. W., Gu, M., . . Koblish, M. 2009. Spirocyclic delta opioid receptor agonists for the treatment of pain: discovery of $N$, $\mathrm{N}$-diethyl-3-hydroxy-4-(spiro [chromene-2, 4'-piperidine]-4-yl) benzamide (ADL5747). Journal of medicinal chemistry, 52(18), 5685-5702.

Le Merrer, J., Becker, J. A., Befort, K., \& Kieffer, B. L. 2009. Reward processing by the opioid system in the brain. Physiological reviews, 89(4), 1379-1412.

Le Merrer, J., Plaza-Zabala, A., Del Boca, C., Matifas, A., Maldonado, R., \& Kieffer, B. L. 2011. Deletion of the $\delta$ opioid receptor gene impairs place conditioning but preserves morphine reinforcement. Biological psychiatry, 69(7), 700-703.

Lecca, S., Meye, F. J., \& Mameli, M. 2014. The lateral habenula in addiction and depression: an anatomical, synaptic and behavioral overview. European Journal of Neuroscience, 39(7), 1170-1178.

Lee, B.-M., Kacew, S., \& Kim, H. S. (2017). Lu's basic toxicology: fundamentals, target organs, and risk assessment: CRC press.

Lees, P., Cunningham, F., \& Elliott, J. 2004. Principles of pharmacodynamics and their applications in veterinary pharmacology. Journal of veterinary pharmacology and therapeutics, 27(6), 397-414

Leri, F., \& Franklin, K. B. 2000. Diazepam in the ventral striatum dissociates dopamine-dependent and dopamine-independent place conditioning. Neuroreport, 11(11), 2553-2556.

Leyton, M., \& Vezina, P. 2013. Striatal ups and downs: their roles in vulnerability to addictions in humans. Neuroscience \& Biobehavioral Reviews, 37(9), 1999-2014. 
Li, L.-B., Chen, N., Ramamoorthy, S., Chi, L., Cui, X.-N., Wang, L. C., \& Reith, M. E. 2004. The role of $\mathrm{N}$-glycosylation in function and surface trafficking of the human dopamine transporter. Journal of Biological Chemistry.

Li, S., Zhu, J., Chen, C., Chen, Y., Deriel, J., Ashby, B., \& Liu-Chen, L. 1993. Molecular cloning and expression of a rat $\mathrm{k}$ opioid receptor. Biochemical Journal, 295(3), 629-633.

Li, Y., Lefever, M. R., Muthu, D., Bidlack, J. M., Bilsky, E. J., \& Polt, R. 2012. Opioid glycopeptide analgesics derived from endogenous enkephalins and endorphins. Future medicinal chemistry, 4(2), 205-226.

Lindholm, S., Werme, M., Brené, S., \& Franck, J. 2001. The selective k-opioid receptor agonist U50, $488 \mathrm{H}$ attenuates voluntary ethanol intake in the rat. Behavioural brain research, 120(2), 137-146.

Linz, K., Christoph, T., Tzschentke, T. M., Koch, T., Schiene, K., Gautrois, M., . . Englberger, W. 2014. Cebranopadol: a novel potent analgesic nociceptin/orphanin FQ peptide and opioid receptor agonist. Journal of Pharmacology and Experimental Therapeutics, 349(3), 535548.

Liu-Chen, L.-Y. 2004. Agonist-induced regulation and trafficking of $\mathrm{k}$ opioid receptors. Life sciences, 75(5), 511-536.

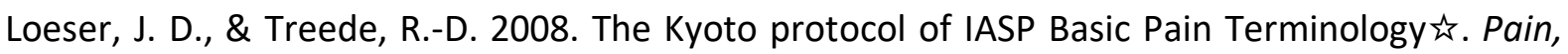
137(3), 473-477.

Logrip, M. L., Janak, P. H., \& Ron, D. 2009. Blockade of ethanol reward by the kappa opioid receptor agonist U50, 488H. Alcohol, 43(5), 359-365.

Lowery, J. J., Raymond, T. J., Giuvelis, D., Bidlack, J. M., Polt, R., \& Bilsky, E. J. 2011. In vivo characterization of MMP-2200, a mixed $\delta / \mu$ opioid agonist, in mice. Journal of Pharmacology and Experimental Therapeutics, 336(3), 767-778.

Lutz, P.-E., \& Kieffer, B. L. 2013a. Opioid receptors: distinct roles in mood disorders. Trends in neurosciences, 36(3), 195-206.

Lutz, P. E., \& Kieffer, B. L. 2013b. The multiple facets of opioid receptor function: implications for addiction. Curr Opin Neurobiol, 23(4), 473-479. doi:10.1016/j.conb.2013.02.005

Lynch, W. C., \& Burns, G. 1990. Opioid effects on intake of sweet solutions depend both on prior drug experience and on prior ingestive experience. Appetite, 15(1), 23-32.

Magrassi, L., Purves, D., \& Lichtman, J. W. 1987. Fluorescent probes that stain living nerve terminals. Journal of Neuroscience, 7(4), 1207-1214.

Mague, S. D., Pliakas, A. M., Todtenkopf, M. S., Tomasiewicz, H. C., Zhang, Y., Stevens, W. C., . . Carlezon, W. A. 2003. Antidepressant-like effects of $\mathrm{k}$-opioid receptor antagonists in the forced swim test in rats. Journal of Pharmacology and Experimental Therapeutics, 305(1), 323-330.

Majumdar, S., Burgman, M., Haselton, N., Grinnell, S., Ocampo, J., Pasternak, A. R., \& Pasternak, G. W. 2011. Generation of novel radiolabeled opiates through site-selective iodination. Bioorganic \& medicinal chemistry letters, 21(13), 4001-4004.

Majumdar, S., \& Devi, L. A. 2018. Strategy for making safer opioids bolstered. Nature, 553(7688), 286-288.

Mansson, E., Bare, L., \& Yang, D. 1994. Isolation of a human к opioid receptor cDNA from placenta. Biochemical and biophysical research communications, 202(3), 1431-1437.

Mantsch, J. R., Li, S.-J., Risinger, R., Awad, S., Katz, E., Baker, D. A., \& Yang, Z. 2007. Levotetrahydropalmatine attenuates cocaine self-administration and cocaine-induced reinstatement in rats. Psychopharmacology, 192(4), 581-591.

Manzardo, A., Stein, L., \& Belluzzi, J. 2002. Rats prefer cocaine over nicotine in a two-lever selfadministration choice test. Brain research, 924(1), 10-19. 
Marchand, S. 2008. The physiology of pain mechanisms: from the periphery to the brain. Rheumatic disease clinics of North America, 34(2), 285-309.

Marinova, Z., Vukojevic, V., Surcheva, S., Yakovleva, T., Cebers, G., Pasikova, N., . . . Hallberg, M. 2005. Translocation of dynorphin neuropeptides across the plasma membrane: a putative mechanism of signal transmission. Journal of Biological Chemistry.

Markou, A., Weiss, F., Gold, L. H., Caine, S. B., Schulteis, G., \& Koob, G. F. 1993. Animal models of drug craving. Psychopharmacology, 112(2-3), 163-182.

Marlatt, G. A. 1996. Models of relapse and relapse prevention: A commentary.

Marshall, J., O'dell, S., Navarrete, R., \& Rosenstein, A. 1990. Dopamine high-affinity transport site topography in rat brain: major differences between dorsal and ventral striatum. Neuroscience, 37(1), 11-21.

Martin, T. J., Kim, S. A., Cannon, D. G., Sizemore, G. M., Bian, D., Porreca, F., \& Smith, J. E. 2000. Antagonism of $\delta 2$-opioid receptors by naltrindole-5'-isothiocyanate attenuates heroin self-administration but not antinociception in rats. Journal of Pharmacology and Experimental Therapeutics, 294(3), 975-982.

Martinez, D., Broft, A., Foltin, R. W., Slifstein, M., Hwang, D.-R., Huang, Y., . . Kleber, H. D. 2004. Cocaine dependence and $D 2$ receptor availability in the functional subdivisions of the striatum: relationship with cocaine-seeking behavior. Neuropsychopharmacology, 29(6), 1190.

Mason, K., Hewitt, A., \& Stefanogiannis, N. (2010). Drug Use in New Zealand: Key Results of the 2007/08 NZ Drugs \& Alcohol Survey: Wellington: Ministry of Health.

Matsumoto, M., Inoue, M., Hald, A., Xie, W., \& Ueda, H. 2006. Inhibition of paclitaxel-induced Afiber hypersensitization by gabapentin. Journal of Pharmacology and Experimental Therapeutics, 318(2), 735-740.

Mazei, M., Pluto, C., Kirkbride, B., \& Pehek, E. 2002. Effects of catecholamine uptake blockers in the caudate-putamen and subregions of the medial prefrontal cortex of the rat. Brain research, 936(1-2), 58-67.

McFadden, M., \& Consultancy, D. M. (2016). The New Zealand Drug Harm Index 2016: Ministry of Health.

McLaughlin, J. P., Land, B. B., Li, S., Pintar, J. E., \& Chavkin, C. 2006. Prior activation of kappa opioid receptors by $U 50,488$ mimics repeated forced swim stress to potentiate cocaine place preference conditioning. Neuropsychopharmacology, 31(4), 787-794.

McLaughlin, J. P., Marton-Popovici, M., \& Chavkin, C. 2003. k opioid receptor antagonism and prodynorphin gene disruption block stress-induced behavioral responses. Journal of Neuroscience, 23(13), 5674-5683.

McLaughlin, J. P., Myers, L. C., Zarek, P. E., Caron, M. G., Lefkowitz, R. J., Czyzyk, T. A., . . Chavkin, C. 2004. Prolonged kappa opioid receptor phosphorylation mediated by G-protein receptor kinase underlies sustained analgesic tolerance. Journal of Biological Chemistry, 279(3), 1810-1818.

McLennan, G. P., Kiss, A., Miyatake, M., Belcheva, M. M., Chambers, K. T., Pozek, J. J., . . Coscia, C. J. 2008. Kappa opioids promote the proliferation of astrocytes via $G \beta \gamma$ and $\beta$-arrestin 2dependent MAPK-mediated pathways. Journal of neurochemistry, 107(6), 1753-1765.

Mei, F., Mayoral, S. R., Nobuta, H., Wang, F., Desponts, C., Lorrain, D. S., . . Whistler, J. 2016. Identification of the kappa-opioid receptor as a therapeutic target for oligodendrocyte remyelination. Journal of Neuroscience, 36(30), 7925-7935.

Meil, W., \& See, R. 1996. Conditioned cued recovery of responding following prolonged withdrawal from self-administered cocaine in rats: an animal model of relapse. Behavioural pharmacology. 
Mello, N. K., Mendelson, J. H., Sholar, M. B., Jaszyna-Gasior, M., Goletiani, N., \& Siegel, A. J. 2005. Effects of the mixed mu/kappa opioid nalbuphine on cocaine-induced changes in subjective and cardiovascular responses in men. Neuropsychopharmacology, 30(3), 618.

Mello, N. K., \& Negus, S. S. 1996. Preclinical evaluation of pharmacotherapies for treatment of cocaine and opioid abuse using drug self-administration procedures. Neuropsychopharmacology, 14(6), 375.

Mello, N. K., \& Negus, S. S. 1998. Effects of kappa opioid agonists on cocaine-and food-maintained responding by rhesus monkeys. Journal of Pharmacology and Experimental Therapeutics, 286(2), 812-824.

Mello, N. K., \& Negus, S. S. 2000. Interactions between kappa opioid agonists and cocaine: preclinical studies. Annals of the New York Academy of Sciences, 909(1), 104-132.

Meng, F., Xie, G.-X., Thompson, R. C., Mansour, A., Goldstein, A., Watson, S. J., \& Akil, H. 1993. Cloning and pharmacological characterization of a rat kappa opioid receptor. Proceedings of the National Academy of Sciences, 90(21), 9954-9958.

Meshul, C., \& McGinty, J. 2000. Kappa opioid receptor immunoreactivity in the nucleus accumbens and caudate-putamen is primarily associated with synaptic vesicles in axons. Neuroscience, 96(1), 91-99.

Miaskowski, C., Taiwo, Y. O., \& Levine, J. D. 1990. $\mathrm{k}$-and $\delta$-opioid agonists synergize to produce potent analgesia. Brain research, 509(1), 165-168.

Michel, M. C., \& Charlton, S. J. 2018. Biased agonism in drug discovery-is it too soon to choose a path? Molecular pharmacology, mol. 117.110890.

Mika, J., Przewłocki, R., \& Przewłocka, B. 2001. The role of $\delta$-opioid receptor subtypes in neuropathic pain. European journal of pharmacology, 415(1), 31-37.

Millan, M. J. 1999. The induction of pain: an integrative review. Progress in neurobiology, 57(1), 1-164.

Miller, G. M. 2011. The emerging role of trace amine-associated receptor 1 in the functional regulation of monoamine transporters and dopaminergic activity. Journal of neurochemistry, 116(2), 164-176.

Milligan, E. D., \& Watkins, L. R. 2009. Pathological and protective roles of glia in chronic pain. Nature reviews neuroscience, 10(1), 23.

Minami, M., Toya, T., Katao, Y., Maekawa, K., Nakamura, S., Onogi, T., ... Satoh, M. 1993. Cloning and expression of a cDNA for the rat k-opioid receptor. FEBS letters, 329(3), 291-295.

Missale, C., Nash, S. R., Robinson, S. W., Jaber, M., \& Caron, M. G. 1998. Dopamine receptors: from structure to function. Physiological reviews, 78(1), 189-225.

Mogil, J. S. 2012. Sex differences in pain and pain inhibition: multiple explanations of a controversial phenomenon. Nature Reviews Neuroscience, 13(12), 859.

Molendijk, M. L., \& de Kloet, E. R. 2015. Immobility in the forced swim test is adaptive and does not reflect depression. Psychoneuroendocrinology, 62, 389-391.

Mols, F., Beijers, T., Vreugdenhil, G., \& van de Poll-Franse, L. 2014. Chemotherapy-induced peripheral neuropathy and its association with quality of life: a systematic review. Supportive Care in Cancer, 22(8), 2261-2269.

Morani, A. S., \& al, e. 2012. A single injection of a novel kappa opioid receptor agonist salvinorin A attenuates the expression of cocaine-induced behavioral sensitization in rats. Behav Pharmacol, 23(2), 162-170. doi:10.1097/FBP.0b013e3283512c1e

Morani, A. S., Ewald, A., Prevatt-Smith, K. M., Prisinzano, T. E., \& Kivell, B. M. 2013. The 2-methoxy methyl analogue of salvinorin $A$ attenuates cocaine-induced drug seeking and sucrose reinforcements in rats. Eur J Pharmacol, 720(1-3), 69-76. doi:10.1016/j.ejphar.2013.10.050 
Morani, A. S., Kivell, B., Prisinzano, T. E., \& Schenk, S. 2009. Effect of kappa-opioid receptor agonists U69593, U50488H, spiradoline and salvinorin A on cocaine-induced drug-seeking in rats. Pharmacol Biochem Behav, 94(2), 244-249. doi:10.1016/j.pbb.2009.09.002

Morani, A. S., Schenk, S., Prisinzano, T. E., \& Kivell, B. 2012. Single injection of novel kappa opioid receptor agonist salvinorin $A$ attenuates expression of cocaine induced behavioral sensitization in rats. Behavioural pharmacology, 23(2), 162.

Mori, T., Kanbara, T., Harumiya, M., Iwase, Y., Masumoto, A., Komiya, S., . . Sakaguchi, G. 2014. Establishment of opioid-induced rewarding effects under oxaliplatin-and Paclitaxelinduced neuropathy in rats. Journal of pharmacological sciences, 126(1), 47-55.

Morinville, A., Cahill, C. M., Esdaile, M. J., Aibak, H., Collier, B., Kieffer, B. L., \& Beaudet, A. 2003. Regulation of $\delta$-opioid receptor trafficking via $\mu$-opioid receptor stimulation: evidence from $\mu$-opioid receptor knock-out mice. Journal of Neuroscience, 23(12), 4888-4898.

Morón, J. A., Brockington, A., Wise, R. A., Rocha, B. A., \& Hope, B. T. 2002. Dopamine uptake through the norepinephrine transporter in brain regions with low levels of the dopamine transporter: evidence from knock-out mouse lines. Journal of Neuroscience, 22(2), 389395.

Morphy, R., Kay, C., \& Rankovic, Z. 2004. From magic bullets to designed multiple ligands. Drug discovery today, 9(15), 641-651.

Morphy, R., \& Rankovic, Z. 2009. Designing multiple ligands-medicinal chemistry strategies and challenges. Current pharmaceutical design, 15(6), 587-600.

Mosberg, H. I., Yeomans, L., Anand, J. P., Porter, V., Sobczyk-Kojiro, K., Traynor, J. R., \& Jutkiewicz, E. M. 2014. Development of a bioavailable $\mu$ opioid receptor (MOPr) agonist, $\delta$ opioid receptor (DOPr) antagonist peptide that evokes antinociception without development of acute tolerance. Journal of medicinal chemistry, 57(7), 3148-3153.

Mura, A., \& Feldon, J. 2003. Spatial learning in rats is impaired after degeneration of the nigrostriatal dopaminergic system. Movement disorders: official journal of the Movement Disorder Society, 18(8), 860-871.

Nadal, X., Baños, J. E., Kieffer, B. L., \& Maldonado, R. 2006. Neuropathic pain is enhanced in $\delta-$ opioid receptor knockout mice. European Journal of Neuroscience, 23(3), 830-834.

Nader, M. A., Morgan, D., Gage, H. D., Nader, S. H., Calhoun, T. L., Buchheimer, N., . . Mach, R. H. 2006. PET imaging of dopamine D2 receptors during chronic cocaine self-administration in monkeys. Nature neuroscience, 9(8), 1050.

Nakao, K., Hirakata, M., Miyamoto, Y., Kainoh, M., Wakasa, Y., \& Yanagita, T. 2016. Nalfurafine hydrochloride, a selective $\mathrm{K}$ opioid receptor agonist, has no reinforcing effect on intravenous self-administration in rhesus monkeys. Journal of pharmacological sciences, 130(1), 8-14.

National Institute On Drug Abuse, U. S. A. (2008). Addiction science: From molecules to managed care. Retrieved from www.drugabuse.gov.

Naylor, J., Prisinzano, T., \& Freeman, K. 2015. Self-administration of oxycodone alone or as a mixture with the kappa agonist, salvinorin a, by monkeys under a progressive ratio schedule of reinforcement. Drug \& Alcohol Dependence, 146, e48-e49.

Negus, S. S. 2004. Effects of the kappa opioid agonist U50, 488 and the kappa opioid antagonist nor-binaltorphimine on choice between cocaine and food in rhesus monkeys. Psychopharmacology, 176(2), 204-213.

Negus, S. S., Mello, N. K., Portoghese, P. S., \& Lin, C.-E. 1997. Effects of kappa opioids on cocaine self-administration by rhesus monkeys. Journal of Pharmacology and Experimental Therapeutics, 282(1), 44-55. 
Negus, S. S., Mello, N. K., Portoghese, P. S., Lukas, S. E., \& Mendelson, J. H. 1995. Role of delta opioid receptors in the reinforcing and discriminative stimulus effects of cocaine in rhesus monkeys. Journal of Pharmacology and Experimental Therapeutics, 273(3), 1245-1256.

Negus, S. S., Schrode, K., \& Stevenson, G. W. 2008. Mu/kappa opioid interactions in rhesus monkeys: Implications for analgesia and abuse liability. Experimental and clinical psychopharmacology, 16(5), 386.

Nestler, E. J. 2001. Molecular basis of long-term plasticity underlying addiction. Nature reviews neuroscience, 2(2), 119.

$\mathrm{Ng}$, K. F. J., Tsui, S. L., \& Chan, W. S. 2002. Prevalence of common chronic pain in Hong Kong adults. The Clinical journal of pain, 18(5), 275-281.

O'Brien, C. P., Childress, A. R., McLELLAN, A. T., \& Ehrman, R. 1992. Classical Conditioning in DrugDependent Humans a. Annals of the New York Academy of Sciences, 654(1), 400-415.

O'Hara, C. M., Uhland-Smith, A., O'Malley, K. L., \& Todd, R. D. 1996. Inhibition of dopamine synthesis by dopamine D2 and D3 but not D4 receptors. Journal of Pharmacology and Experimental Therapeutics, 277(1), 186-192.

O'Malley, S. S., Jaffe, A. J., Chang, G., Schottenfeld, R. S., Meyer, R. E., \& Rounsaville, B. 1992. Naltrexone and coping skills therapy for alcohol dependence: a controlled study. Archives of general psychiatry, 49(11), 881-887.

O'Brien, C. P. 2005. Anticraving medications for relapse prevention: a possible new class of psychoactive medications. American Journal of Psychiatry, 162(8), 1423-1431.

O'connor, E. C., Chapman, K., Butler, P., \& Mead, A. N. 2011. The predictive validity of the rat selfadministration model for abuse liability. Neuroscience \& Biobehavioral Reviews, 35(3), 912-938.

Okie, S. 2010. A flood of opioids, a rising tide of deaths. New England Journal of Medicine, 363(21), 1981-1985.

Ong, E. W., Holdridge, S. V., Sutherland, K. A., Armstrong, S. A., Taylor, A. M., Xue, L., \& Cahill, C. M. 2016. Neuropathic pain unmasks delta opioid receptor-mediated analgesia. The FASEB Journal, 30(1_supplement), 928.921-928.921.

Oz, M., Libby, T., Kivell, B., Jaligam, V., Ramamoorthy, S., \& Shippenberg, T. S. 2010. Real-time, spatially resolved analysis of serotonin transporter activity and regulation using the fluorescent substrate, ASP+. Journal of neurochemistry, 114(4), 1019-1029.

Panlilio, L. V., \& Goldberg, S. R. 2007. Self-administration of drugs in animals and humans as a model and an investigative tool. Addiction, 102(12), 1863-1870.

Paris, J. J., Reilley, K. J., \& McLaughlin, J. P. 2011. Kappa opioid receptor-mediated disruption of novel object recognition: Relevance for psychostimulant treatment. Journal of addiction research \& therapy.

Pascual, D., Goicoechea, C., Burgos, E., \& Martín, M. I. 2010. Antinociceptive effect of three common analgesic drugs on peripheral neuropathy induced by paclitaxel in rats. Pharmacology Biochemistry and Behavior, 95(3), 331-337.

Pathan, H., \& Williams, J. 2012. Basic opioid pharmacology: an update. British journal of pain, 6(1), 11-16.

Paton, K., Kumar, N., Crowley, R., Harper, J., Prisinzano, T., \& Kivell, B. 2017. The analgesic and anti-inflammatory effects of Salvinorin A analogue $\beta$-tetrahydropyran Salvinorin B in mice. European Journal of Pain.

Paton, K. F. 2018. Investigating the antinociceptive effects of $\mathrm{N}$-docosahexaenoyl ethanolamine and novel kappa opioid receptor agonists.

Patwardhan, A. M., Berg, K. A., Akopain, A. N., Jeske, N. A., Gamper, N., Clarke, W. P., \& Hargreaves, K. M. 2005. Bradykinin-induced functional competence and trafficking of the $\delta$-opioid receptor in trigeminal nociceptors. Journal of Neuroscience, 25(39), 8825-8832. 
Pecina, S., \& Berridge, K. C. 2005. Hedonic hot spot in nucleus accumbens shell: where do $\mu-$ opioids cause increased hedonic impact of sweetness? Journal of neuroscience, 25(50), 11777-11786.

Peckys, D., \& Landwehrmeyer, G. 1999. Expression of mu, kappa, and delta opioid receptor messenger RNA in the human CNS: a 33P in situ hybridization study. Neuroscience, 88(4), 1093-1135.

Peng, J., Sarkar, S., \& Chang, S. L. 2012. Opioid receptor expression in human brain and peripheral tissues using absolute quantitative real-time RT-PCR. Drug and alcohol dependence, 124(3), 223-228.

Perlikowska, R., Piekielna, J., Gentilucci, L., De Marco, R., Cerlesi, M. C., Calo, G., . . Janecka, A. 2016. Synthesis of mixed MOR/KOR efficacy cyclic opioid peptide analogs with antinociceptive activity after systemic administration. European journal of medicinal chemistry, 109, 276-286.

Perrine, S. A., Hoshaw, B. A., \& Unterwald, E. M. 2006. Delta opioid receptor ligands modulate anxiety-like behaviors in the rat. British journal of pharmacology, 147(8), 864-872.

Pettit, H. O., Ettenberg, A., Bloom, F. E., \& Koob, G. F. 1984. Destruction of dopamine in the nucleus accumbens selectively attenuates cocaine but not heroin self-administration in rats. Psychopharmacology, 84(2), 167-173.

Pfaus, J., Damsma, G., Nomikos, G. G., Wenkstern, D., Blaha, C., Phillips, A., \& Fibiger, H. 1990. Sexual behavior enhances central dopamine transmission in the male rat. Brain research, 530(2), 345-348.

Pfeiffer, A., Brantl, V., Herz, A., \& Emrich, H. M. 1986. Psychotomimesis mediated by kappa opiate receptors. Science, 233(4765), 774-776.

Phillips, A. G., \& Fibiger, H. C. 1978. The role of dopamine in maintaining intracranial selfstimulation in the ventral tegmentum, nucleus accumbens, and medial prefrontal cortex. Canadian Journal of Psychology/Revue canadienne de psychologie, 32(2), 58.

Pickens, C. L., Airavaara, M., Theberge, F., Fanous, S., Hope, B. T., \& Shaham, Y. 2011. Neurobiology of the incubation of drug craving. Trends in neurosciences, 34(8), 411-420.

Pickens, R., \& Harris, W. C. 1968. Self-administration of d-amphetamine by rats. Psychopharmacologia, 12(2), 158-163.

Pickens, R., Meisch, R., \& McGuire, L. E. 1967. Methamphetamine reinforcement in rats. Psychonomic Science, 8(9), 371-372.

Pickens, R., \& Thompson, T. 1968. Cocaine-reinforced behavior in rats: effects of reinforcement magnitude and fixed-ratio size. Journal of Pharmacology and Experimental Therapeutics, 161(1), 122-129.

Pifl, C., Drobny, H., Reither, H., Hornykiewicz, O., \& Singer, E. A. 1995. Mechanism of the dopamine-releasing actions of amphetamine and cocaine: plasmalemmal dopamine transporter versus vesicular monoamine transporter. Molecular Pharmacology, 47(2), 368-373.

Pliakas, A. M., Carlson, R. R., Neve, R. L., Konradi, C., Nestler, E. J., \& Carlezon, W. A. 2001. Altered responsiveness to cocaine and increased immobility in the forced swim test associated with elevated cAMP response element-binding protein expression in nucleus accumbens. Journal of Neuroscience, 21(18), 7397-7403.

Pogozheva, I. D., Przydzial, M. J., \& Mosberg, H. I. 2005. Homology modeling of opioid receptorligand complexes using experimental constraints. The AAPS journal, 7(2), E434-E448.

Porsolt, R., Le Pichon, M., \& Jalfre, M. 1977. Depression: a new animal model sensitive to antidepressant treatments. Nature, 266(5604), 730. 
Potter, D. N., Damez-Werno, D., Carlezon Jr, W. A., Cohen, B. M., \& Chartoff, E. H. 2011. Repeated exposure to the $\mathrm{K}$-opioid receptor agonist salvinorin A modulates extracellular signalregulated kinase and reward sensitivity. Biological psychiatry, 70(8), 744-753.

Povlock, S. L., \& Schenk, J. O. 1997. A multisubstrate kinetic mechanism of dopamine transport in the nucleus accumbens and its inhibition by cocaine. Journal of neurochemistry, 69(3), 1093-1105.

Pradhan, A., Smith, M., McGuire, B., Evans, C., \& Walwyn, W. 2013. Chronic inflammatory injury results in increased coupling of delta opioid receptors to voltage-gated $\mathrm{Ca} 2+$ channels. Molecular pain, 9(1), 8.

Pradhan, A. A., Befort, K., Nozaki, C., Gavériaux-Ruff, C., \& Kieffer, B. L. 2011. The delta opioid receptor: an evolving target for the treatment of brain disorders. Trends in pharmacological sciences, 32(10), 581-590.

Prisciandaro, J. J., Joseph, J. E., Myrick, H., McRae-Clark, A. L., Henderson, S., Pfeifer, J., \& Brady, K. T. 2014. The relationship between years of cocaine use and brain activation to cocaine and response inhibition cues. Addiction, 109(12), 2062-2070.

Provencher, B. A., Sromek, A. W., Li, W., Russell, S., Chartoff, E., Knapp, B. I., . . Neumeyer, J. L. 2013. Synthesis and pharmacological evaluation of aminothiazolomorphinans at the mu and kappa opioid receptors. Journal of medicinal chemistry, 56(21), 8872-8878.

Qi, J., Heyman, J., Sheldon, R., Koslo, R., \& Porreca, F. 1990. Mu antagonist and kappa agonist properties of beta-funaltrexamine (beta-FNA) in vivo: long-lasting spinal analgesia in mice. Journal of Pharmacology and Experimental Therapeutics, 252(3), 1006-1011.

Raiteri, M., Del Carmine, R., Bertollini, A., \& Levi, G. 1977. Effect of sympathomimetic amines on the synaptosomal transport of noradrenaline, dopamine and 5-hydroxytryptamine. European journal of pharmacology, 41(2), 133-143.

Randic, M., Cheng, G., \& Kojic, L. (1995). kappa-opioid receptor agonists modulate excitatory transmission in substantia gelatinosa neurons of the rat spinal cord. Journal of Neuroscience, 15(10), 6809-6826.

Rankovic, Z., Brust, T. F., \& Bohn, L. M. 2016. Biased agonism: An emerging paradigm in GPCR drug discovery. Bioorganic \& medicinal chemistry letters, 26(2), 241-250.

Rao, R. D., Michalak, J. C., Sloan, J. A., Loprinzi, C. L., Soori, G. S., Nikcevich, D. A., . . Wong, G. Y. 2007. Efficacy of gabapentin in the management of chemotherapy-induced peripheral neuropathy. Cancer, 110(9), 2110-2118.

Rasakham, K., \& Liu-Chen, L.-Y. 2011. Sex differences in kappa opioid pharmacology. Life sciences, 88(1-2), 2-16.

Redila, V. A., \& Chavkin, C. 2008. Stress-induced reinstatement of cocaine seeking is mediated by the kappa opioid system. Psychopharmacology, 200(1), 59-70.

Reid, L. D., Glick, S. D., Menkens, K. A., French, E. D., Bilsky, E. J., \& Porreca, F. 1995. Cocaine selfadministration and naltrindole, a delta-selective opioid antagonist. Neuroreport: An International Journal for the Rapid Communication of Research in Neuroscience.

Richards, T. L., \& Zahniser, N. R. 2009. Rapid substrate-induced down-regulation in function and surface localization of dopamine transporters: rat dorsal striatum versus nucleus accumbens. Journal of neurochemistry, 108(6), 1575-1584.

Richfield, E. K. 1991. Quantitative autoradiography of the dopamine uptake complex in rat brain using [3H] GBR 12935: binding characteristics. Brain research, 540(1-2), 1-13.

Riley, A. P., Groer, C. E., Young, D., Ewald, A. W., Kivell, B. M., \& Prisinzano, T. E. 2014. Synthesis and kappa-opioid receptor activity of furan-substituted salvinorin A analogues. Journal of medicinal chemistry, 57(24), 10464-10475. doi:10.1021/jm501521d

Ritz, M. C., Lamb, R., \& Kuhar, M. 1987. Cocaine receptors on dopamine transporters are related to self-administration of cocaine. Science, 237(4819), 1219-1223. 
Roberts, M., \& Rees, H. 1986. The antinociceptive effects of stimulating the pretectal nucleus of the rat. Pain, 25(1), 83-93.

Robinson, T. E., \& Berridge, K. C. 1993. The neural basis of drug craving: an incentive-sensitization theory of addiction. Brain research reviews, 18(3), 247-291.

Robinson, T. E., \& Berridge, K. C. 2001. Incentive-sensitization and addiction. Addiction, 96(1), 103114.

Robinson, T. E., \& Berridge, K. C. 2008. The incentive sensitization theory of addiction: some current issues. Philosophical Transactions of the Royal Society of London B: Biological Sciences, 363(1507), 3137-3146.

Rowan, M. P., Ruparel, N. B., Patwardhan, A. M., Berg, K. A., Clarke, W. P., \& Hargreaves, K. M. 2009. Peripheral delta opioid receptors require priming for functional competence in vivo. European journal of pharmacology, 602(2-3), 283-287.

Rozenfeld, R., Abul-Husn, N. S., Gomez, I., \& Devi, L. A. 2007. An emerging role for the delta opioid receptor in the regulation of mu opioid receptor function. The Scientific World Journal, 7, 64-73.

Rudd, R. A. 2016. Increases in Drug and Opioid-Involved Overdose Deaths-United States, 20102015. MMWR. Morbidity and Mortality Weekly Report, 65.

Rüedi-Bettschen, D., Rowlett, J. K., Spealman, R. D., \& Platt, D. M. 2010. Attenuation of cocaineinduced reinstatement of drug seeking in squirrel monkeys: kappa opioid and serotonergic mechanisms. Psychopharmacology, 210(2), 169-177.

Ruiz-Garcia, A., Bermejo, M., Moss, A., \& Casabo, V. G. 2008. Pharmacokinetics in drug discovery. Journal of pharmaceutical sciences, 97(2), 654-690.

Saadé, N. E., \& Jabbur, S. J. 1985. Dorsal column influences, through the brainstem, on spinal nociceptive input. Development, organization, and processing in somatosensory pathways, New York: Alan Liss, 367-373.

Saitoh, A., Kimura, Y., Suzuki, T., Kawai, K., Nagase, H., \& Kamei, J. 2004. Potential anxiolytic and antidepressant-like activities of SNC80, a selective $\delta$-opioid agonist, in behavioral models in rodents. Journal of pharmacological sciences, 95(3), 374-380.

Sauver, J. L. S., Warner, D. O., Yawn, B. P., Jacobson, D. J., McGree, M. E., Pankratz, J. J., . . Rocca, W. A. (2013). Why patients visit their doctors: assessing the most prevalent conditions in a defined American population. Paper presented at the Mayo Clinic Proceedings.

Schattauer, S. S., Kuhar, J. R., Song, A., \& Chavkin, C. 2017. Nalfurafine is a G-protein biased agonist having significantly greater bias at the human than rodent form of the kappa opioid receptor. Cellular signalling, 32, 59-65.

Scheffel, U., Pögün, S., Stathis, M., Boja, J. W., \& Kuhar, M. J. 1991. In vivo labeling of cocaine binding sites on dopamine transporters with [3H] WIN 35,428. Journal of Pharmacology and Experimental Therapeutics, 257(3), 954-958.

Schenk, J. O. (2002). The functioning neuronal transporter for dopamine: kinetic mechanisms and effects of amphetamines, cocaine and methylphenidate Progress in Drug Research (pp. 111-131): Springer.

Schenk, S., Hely, L., Lake, B., Daniela, E., Gittings, D., \& Mash, D. C. 2007. MDMA selfadministration in rats: acquisition, progressive ratio responding and serotonin transporter binding. European Journal of Neuroscience, 26(11), 3229-3236.

Schenk, S., \& Partridge, B. 2001. Effect of the kappa-opioid receptor agonist, U69593, on reinstatement of extinguished amphetamine self-administration behavior. Pharmacology Biochemistry and Behavior, 68(4), 629-634. doi:http://dx.doi.org/10.1016/S00913057(00)00478-0 
Schenk, S., Partridge, B., \& Shippenberg, T. S. 1999. U69593, a kappa-opioid agonist, decreases cocaine self-administration and decreases cocaine-produced drug-seeking. Psychopharmacology, 144(4), 339-346.

Schenk, S., Partridge, B., \& Shippenberg, T. S. 2000. Reinstatement of extinguished drug-taking behavior in rats: effect of the kappa-opioid receptor agonist, U69593. Psychopharmacology, 151(1), 85-90.

Schiller, P. W., Fundytus, M. E., Merovitz, L., Weltrowska, G., Nguyen, T. M.-D., Lemieux, C., . . . Coderre, T. J. 1999. The opioid $\mu$ agonist/ $\delta$ antagonist DIPP-NH2 [U] produces a potent analgesic effect, no physical dependence, and less tolerance than morphine in rats. Journal of medicinal chemistry, 42(18), 3520-3526.

Schindler, C. W., Panlilio, L. V., \& Goldberg, S. R. 2002. Second-order schedules of drug selfadministration in animals. Psychopharmacology, 163(3), 327-344.

Schultz, W. 2007. Behavioral dopamine signals. Trends in neurosciences, 30(5), 203-210.

Schulz, R., Wehmeyer, A., \& Schulz, K. 2002. Visualizing preference of G protein-coupled receptor kinase 3 for the process of $\mathrm{k}$-opioid receptor sequestration. Molecular pharmacology, 61(6), 1444-1452.

Schwartz, J. W., Blakely, R. D., \& DeFelice, L. J. 2003. Binding and transport in norepinephrine transporters real-time, spatially resolved analysis in single cells using a fluorescent substrate. Journal of Biological Chemistry, 278(11), 9768-9777.

Seip-Cammack, K. M., Young, J. J., Young, M. E., \& Shapiro, M. L. 2017. Partial lesion of the nigrostriatal dopamine pathway in rats impairs egocentric learning but not spatial learning or behavioral flexibility. Behavioral neuroscience, 131(2), 135.

Self, D. W. 2004. Regulation of drug-taking and-seeking behaviors by neuroadaptations in the mesolimbic dopamine system. Neuropharmacology, 47, 242-255.

Self, D. W., \& Nestler, E. J. 1998. Relapse to drug-seeking: neural and molecular mechanisms. Drug \& Alcohol Dependence, 51(1), 49-60.

Self, D. W., Terwilliger, R. Z., Nestler, E. J., \& Stein, L. 1994. Inactivation of Gi and G (o) proteins in nucleus accumbens reduces both cocaine and heroin reinforcement. Journal of Neuroscience, 14(10), 6239-6247.

Selph, S., Carson, S., Fu, R., Thakurta, S., Low, A., \& McDonagh, M. 2011. Drug class review: neuropathic pain. Update, 1.

Sesack, S. R., Hawrylak, V. A., Matus, C., Guido, M. A., \& Levey, A. I. 1998. Dopamine axon varicosities in the prelimbic division of the rat prefrontal cortex exhibit sparse immunoreactivity for the dopamine transporter. Journal of Neuroscience, 18(7), 26972708.

Shaham, Y., Shalev, U., Lu, L., De Wit, H., \& Stewart, J. 2003. The reinstatement model of drug relapse: history, methodology and major findings. Psychopharmacology, 168(1-2), 3-20.

Shaham, Y., \& Stewart, J. 1995. Stress reinstates heroin-seeking in drug-free animals: an effect mimicking heroin, not withdrawal. Psychopharmacology, 119(3), 334-341.

Shalev, U., Grimm, J. W., \& Shaham, Y. 2002. Neurobiology of relapse to heroin and cocaine seeking: a review. Pharmacological reviews, 54(1), 1-42.

Shimada, S., Kitayama, S., Walther, D., \& Uhl, G. 1992. Dopamine transporter mRNA: dense expression in ventral midbrain neurons. Molecular brain research, 13(4), 359-362.

Shippenberg, T., LeFevour, A., \& Chefer, V. 2008. Targeting endogenous mu-and delta-opioid receptor systems for the treatment of drug addiction. CNS \& Neurological Disorders-Drug Targets (Formerly Current Drug Targets-CNS \& Neurological Disorders), 7(5), 442-453.

Shippenberg, T., LeFevour, A., \& Heidbreder, C. 1996. kappa-Opioid receptor agonists prevent sensitization to the conditioned rewarding effects of cocaine. Journal of Pharmacology and Experimental Therapeutics, 276(2), 545-554. 
Shippenberg, T., Zapata, A., \& Chefer, V. 2007. Dynorphin and the pathophysiology of drug addiction. Pharmacology \& therapeutics, 116(2), 306-321.

Shippenberg, T. S., Chefer, V. I., \& Thompson, A. C. 2009. Delta-opioid receptor antagonists prevent sensitization to the conditioned rewarding effects of morphine. Biological psychiatry, 65(2), 169-174.

Shippenberg, T. S., \& Koob, G. F. 2002. Recent advances in animal models of drug addiction and alcoholism. Neuropsychopharmacology: The fifth generation of progress, 13, 81-97.

Shirayama, Y., Ishida, H., Iwata, M., Hazama, G. i., Kawahara, R., \& Duman, R. S. 2004. Stress increases dynorphin immunoreactivity in limbic brain regions and dynorphin antagonism produces antidepressant-like effects. Journal of neurochemistry, 90(5), 1258-1268.

Shook, J. E., Watkins, W. D., \& Camporesi, E. M. 1990. Differential roles of opioid receptors in respiration, respiratory disease, and opiate-induced respiratory depression. Am Rev Respir Dis, 142(4), 895-909.

Simon, L. S. 2012. Relieving pain in America: A blueprint for transforming prevention, care, education, and research. Journal of pain \& palliative care pharmacotherapy, 26(2), 197198.

Simmons, D., \& Self, D. W. 2009. Role of mu-and delta-opioid receptors in the nucleus accumbens in cocaine-seeking behavior. Neuropsychopharmacology, 34(8), 1946.

Simonin, F., Befort, K., Gaveriaux-Ruff, C., Matthes, H., Nappey, V., Lannes, B., . . Kieffer, B. 1994. The human delta-opioid receptor: genomic organization, cDNA cloning, functional expression, and distribution in human brain. Molecular Pharmacology, 46(6), 1015-1021.

Simonin, F., Gaveriaux-Ruff, C., Befort, K., Matthes, H., Lannes, B., Micheletti, G., . . . Kieffer, B. 1995. kappa-Opioid receptor in humans: cDNA and genomic cloning, chromosomal assignment, functional expression, pharmacology, and expression pattern in the central nervous system. Proceedings of the National Academy of Sciences, 92(15), 7006-7010.

Simonson, B. 2011. Investigating the effects of novel kappa opioid receptor agonists on the dopamine transporter.

Simonson, B., Morani, A., Ewald, A., Walker, L., Kumar, N., Simpson, D., . . . Kivell, B. 2015. Pharmacology and anti-addiction effects of the novel $\mathrm{K}$ opioid receptor agonist Mesyl Sal $B$, a potent and long-acting analogue of salvinorin A. British journal of pharmacology, 172(2), 515-531.

Sinha, R., Shaham, Y., \& Heilig, M. 2011. Translational and reverse translational research on the role of stress in drug craving and relapse. Psychopharmacology, 218(1), 69-82.

Sipols, A., Bayer, J., Bennett, R., \& Figlewicz, D. 2002. Intraventricular insulin decreases kappa opioid-mediated sucrose intake in rats. Peptides, 23(12), 2181-2187.

Skoubis, P., Matthes, H., Walwyn, W., Kieffer, B., \& Maidment, N. 2001. Naloxone fails to produce conditioned place aversion in $\mu$-opioid receptor knock-out mice. Neuroscience, 106(4), 757-763.

Slattery, D. A., \& Cryan, J. F. 2012. Using the rat forced swim test to assess antidepressant-like activity in rodents. Nature protocols, 7(6), 1009.

Smith, E., Pang, H., Cirrincione, C., Fleishman, S., Paskett, E., Ahles, T., . . Le-Lindqwister, N. 2013. Alliance for Clinical Trials in Oncology. Effect of duloxetine on pain, function, and quality of life among patients with chemotherapy-induced painful peripheral neuropathy: a randomized clinical trial. Jama, 309(13), 1359-1367.

Smith, J. S., Schindler, A. G., Martinelli, E., Gustin, R. M., Bruchas, M. R., \& Chavkin, C. 2012. Stressinduced activation of the dynorphin/K-opioid receptor system in the amygdala potentiates nicotine conditioned place preference. Journal of Neuroscience, 32(4), 14881495. 
Smith, K. S., Berridge, K. C., \& Aldridge, J. W. 2011. Disentangling pleasure from incentive salience and learning signals in brain reward circuitry. Proceedings of the National Academy of Sciences, 108(27), E255-E264.

Smith, S. B., Crager, S. E., \& Mogil, J. S. 2004. Paclitaxel-induced neuropathic hypersensitivity in mice: responses in 10 inbred mouse strains. Life sciences, 74(21), 2593-2604.

Smith, B. H., Elliott, A. M., Chambers, W. A., Smith, W. C., Hannaford, P. C., \& Penny, K. 2001. The impact of chronic pain in the community. Family practice, 18(3), 292-299.

Sobanski, P., Krajnik, M., Shaqura, M., Bloch-Boguslawska, E., Schäfer, M., \& Mousa, S. A. 2014. The presence of mu-, delta-, and kappa-opioid receptors in human heart tissue. Heart and vessels, 29(6), 855-863.

Sobczak, M., Pilarczyk, A., Jonakowski, M., Jarmuż, A., Sałaga, M., Lipkowski, A. W., \& Fichna, J. 2014. Anti-inflammatory and antinociceptive action of the dimeric enkephalin peptide biphalin in the mouse model of colitis: new potential treatment of abdominal pain associated with inflammatory bowel diseases. Peptides, 60, 102-106.

Solomon, R. L. 1977. Addiction: an opponent-process theory of acquired motivation: the affective dynamics of addiction.

Solomon, R. L., \& Corbit, J. D. 1974. An opponent-process theory of motivation: I. Temporal dynamics of affect. Psychological review, 81(2), 119.

Song, R., Yang, R. F., Wu, N., Su, R. B., Li, J., Peng, X. Q., . . Gardner, E. L. 2012. YQA14: a novel dopamine D3 receptor antagonist that inhibits cocaine self-administration in rats and mice, but not in D3 receptor-knockout mice. Addiction biology, 17(2), 259-273.

Spanagel, R., Herz, A., \& Shippenberg, T. S. 1992. Opposing tonically active endogenous opioid systems modulate the mesolimbic dopaminergic pathway. Proceedings of the National Academy of Sciences, 89(6), 2046-2050.

Spangler, R., Zhou, Y., Maggos, C. E., Schlussman, S. D., Ho, A., \& Kreek, M. J. 1997. Prodynorphin, proenkephalin and $\mathrm{k}$ opioid receptor mRNA responses to acute "binge" cocaine. Molecular brain research, 44(1), 139-142.

Spetea, M., Eans, S. O., Ganno, M. L., Lantero, A., Mairegger, M., Toll, L., . . McLaughlin, J. P. 2017. Selective $\mathrm{k}$ receptor partial agonist HS666 produces potent antinociception without inducing aversion after icv administration in mice. British journal of pharmacology, 174(15), 2444-2456.

Stack, A., Business, Limited, E. R., \& et al. (2009). Costs of harmful and alcohol and other drug use: BERL economics.

Strakowski, S. M., \& Sax, K. W. 1998. Progressive behavioral response to repeated d-amphetamine challenge: further evidence for sensitization in humans. Biological psychiatry, 44(11), 1171-1177.

Stein, C., Millan, M. J., Shippenberg, T. S., Peter, K., \& Herz, A. 1989. Peripheral opioid receptors mediating antinociception in inflammation. Evidence for involvement of $\mathrm{mu}$, delta and kappa receptors. Journal of Pharmacology and Experimental Therapeutics, 248(3), 12691275.

Stein, L. (1964). Self-stimulation of the brain and the central stimulant action of amphetamine. Paper presented at the Federation proceedings.

Sternberg, W. F., Chesler, E. J., Wilson, S. G., \& Mogil, J. S. 2004. Acute progesterone can recruit sex-specific neurochemical mechanisms mediating swim stress-induced and k-opioid analgesia in mice. Hormones and behavior, 46(4), 467-473.

Stewart, J. 2000. Pathways to relapse: the neurobiology of drug-and stress-induced relapse to drug-taking. Journal of Psychiatry and Neuroscience, 25(2), 125. 
Stretch, R., Gerber, G. J., \& Wood, S. M. 1971. Factors affecting behavior maintained by responsecontingent intravenous infusions of amphetamine in squirrel monkeys. Canadian journal of physiology and pharmacology, 49(6), 581-589.

Stromberg, M. F., Casale, M., Volpicelli, L., Volpicelli, J. R., \& O'BRIEN, C. P. 1998. A comparison of the effects of the opioid antagonists naltrexone, naltrindole, and $\beta$-funaltrexamine on ethanol consumption in the rat. Alcohol, 15(4), 281-289.

Sufka, K. J., Loria, M. J., Lewellyn, K., Zjawiony, J. K., Ali, Z., Abe, N., \& Khan, I. A. 2014. The effect of Salvia divinorum and Mitragyna speciosa extracts, fraction and major constituents on place aversion and place preference in rats. Journal of ethnopharmacology, 151(1), 361364.

Sukhtankar, D. D., Zaveri, N. T., Husbands, S. M., \& Ko, M.-C. 2013. Effects of spinally administered bifunctional nociceptin/orphanin FQ peptide receptor/ $\mu$-opioid receptor ligands in mouse models of neuropathic and inflammatory pain. Journal of Pharmacology and Experimental Therapeutics, 346(1), 11-22.

Sun, J.-f., Wang, Y.-h., Li, F.-y., Lu, G., Tao, Y.-m., Cheng, Y., . . Neumeyer, J. L. 2010. Effects of ATPM-ET, a novel $\mathrm{k}$ agonist with partial $\mu$ activity, on physical dependence and behavior sensitization in mice. Acta Pharmacologica Sinica, 31(12), 1547.

Suzuki, T., Izumimoto, N., Takezawa, Y., Fujimura, M., Togashi, Y., Nagase, H., . . Endoh, T. 2004. Effect of repeated administration of TRK-820, a k-opioid receptor agonist, on tolerance to its antinociceptive and sedative actions. Brain Research, 995(2), 167-175. doi:http://dx.doi.org/10.1016/j. brainres.2003.09.057

Suzuki, T., \& Misawa, M. 1997. Opioid receptor types and dependence. Nihon yakurigaku zasshi. Folia pharmacologica Japonica, 109(4), 165-174.

Suzuki, T., Narita, M., Takahashi, Y., Misawa, M., \& Nagase, H. 1992a. Effects of norbinaltorphimine on the development of analgesic tolerance to and physical dependence on morphine. European journal of pharmacology, 213(1), 91-97.

Suzuki, T., Shiozaki, Y., Masukawa, Y., Misawa, M., \& Nagase, H. 1992b. The role of mu-and kappaopioid receptors in cocaine-induced conditioned place preference. The Japanese Journal of Pharmacology, 58(4), 435-442.

Suzuki, T., Tsuji, M., Mori, T., Misawa, M., Endoh, T., \& Nagase, H. 1996. Effect of the highly selective and nonpeptide delta opioid receptor agonist TAN-67 on the morphine-induced place preference in mice. Journal of Pharmacology and Experimental Therapeutics, 279(1), 177-185.

Svingos, A. L., Chavkin, C., Colago, E. E., \& Pickel, V. M. 2001. Major coexpression of K-opioid receptors and the dopamine transporter in nucleus accumbens axonal profiles. Synapse, 42(3), 185-192.

Swain, N., \& Johnson, M. 2014. Chronic pain in New Zealand: a community sample. The New Zealand Medical Journal, 127(1388), 21-30.

Swanson, L. 1982. The projections of the ventral tegmental area and adjacent regions: a combined fluorescent retrograde tracer and immunofluorescence study in the rat. Brain research bulletin, 9(1-6), 321-353.

Tang, L., Todd, R. D., \& O'Malley, K. L. 1994. Dopamine D2 and D3 receptors inhibit dopamine release. Journal of Pharmacology and Experimental Therapeutics, 270(2), 475-479.

Tang, Y., Yang, J., Lunzer, M. M., Powers, M. D., \& Portoghese, P. S. 2010. A k opioid pharmacophore becomes a spinally selective $\mathrm{k}-\delta$ agonist when modified with a basic extender arm. ACS medicinal chemistry letters, 2(1), 7-10.

Tao, P.-L., Hwang, C.-L., \& Chen, C.-Y. 1994. U-50,488 blocks the development of morphine tolerance and dependence at a very low dose in guinea pigs. European journal of pharmacology, 256(3), 281-286. 
Tao, Y.-M., Li, Q.-L., Zhang, C.-F., Xu, X.-J., Chen, J., Ju, Y.-W., . . . Liu, J.-G. 2008. LPK-26, a novel kopioid receptor agonist with potent antinociceptive effects and low dependence potential. European journal of pharmacology, 584(2), 306-311.

Tappe-Theodor, A., \& Kuner, R. 2014. Studying ongoing and spontaneous pain in rodentschallenges and opportunities. European Journal of Neuroscience, 39(11), 1881-1890.

Taussig, R., Iniguez-Lluhi, J. A., \& Gilman, A. G. 1993. Inhibition of adenylyl cyclase by Gi alpha. Science, 261(5118), 218-221.

Taylor, A., Roberts, K., Pradhan, A., Akbari, H., Walwyn, W., Lutfy, K., . . . Evans, C. 2015. Antinociception mediated by a $\mathrm{K}$ opioid receptor agonist is blocked by a $\delta$ receptor agonist. British journal of pharmacology, 172(2), 691-703.

Tejeda, H. A., Counotte, D. S., Oh, E., Ramamoorthy, S., Schultz-Kuszak, K. N., Bäckman, C. M., .. . Shippenberg, T. S. 2013. Prefrontal cortical kappa-opioid receptor modulation of local neurotransmission and conditioned place aversion. Neuropsychopharmacology, 38(9), 1770.

Tejedor-Real, P., Micó, J. A., Smadja, C., Maldonado, R., Roques, B. P., \& Gibert-Rahola, J. 1998. Involvement of $\delta$-opioid receptors in the effects induced by endogenous enkephalins on learned helplessness model. European journal of pharmacology, 354(1), 1-7.

Thangamani, D., Edafiogho, I. O., \& Masocha, W. 2013. The anticonvulsant enaminone E139 attenuates paclitaxel-induced neuropathic pain in rodents. The Scientific World Journal, 2013.

Thomas, M. J., Beurrier, C., Bonci, A., \& Malenka, R. C. 2001. Long-term depression in the nucleus accumbens: a neural correlate of behavioral sensitization to cocaine. Nature neuroscience, 4(12), 1217.

Thompson, A. C., Zapata, A., Justice, J. B., Vaughan, R. A., Sharpe, L. G., \& Shippenberg, T. S. 2000. $\mathrm{K}$-Opioid receptor activation modifies dopamine uptake in the nucleus accumbens and opposes the effects of cocaine. Journal of Neuroscience, 20(24), 9333-9340.

Thompson, T., \& Schuster, C. R. 1964. Morphine self-administration, food-reinforced, and avoidance behaviors in rhesus monkeys. Psychopharmacology, 5(2), 87-94.

Thorn, D. A., Zhang, Y., Peng, B.-W., Winter, J. C., \& Li, J.-X. 2011. Effects of imidazoline 12 receptor ligands on morphine-and tramadol-induced antinociception in rats. European journal of pharmacology, 670(2-3), 435-440.

Toblin, R. L., Mack, K. A., Perveen, G., \& Paulozzi, L. J. 2011. A population-based survey of chronic pain and its treatment with prescription drugs. Pain, 152(6), 1249-1255.

Todtenkopf, M. S., Marcus, J. F., Portoghese, P. S., \& Carlezon, W. A. 2004. Effects of k-opioid receptor ligands on intracranial self-stimulation in rats. Psychopharmacology, 172(4), 463470.

Toll, L., Khroyan, T., Polgar, W., Husbands, S. M., \& Zaveri, N. 2013. Pharmacology of mixed NOP/Mu ligands.

Toll, L., Khroyan, T. V., Polgar, W. E., Jiang, F., Olsen, C., \& Zaveri, N. T. 2009. Comparison of the antinociceptive and antirewarding profiles of novel bifunctional nociceptin receptor/ $\mu$ opioid receptor ligands: implications for therapeutic applications. Journal of Pharmacology and Experimental Therapeutics, 331(3), 954-964.

Tomasiewicz, H. C., Todtenkopf, M. S., Chartoff, E. H., Cohen, B. M., \& Carlezon Jr, W. A. 2008. The kappa-opioid agonist U69, 593 blocks cocaine-induced enhancement of brain stimulation reward. Biological psychiatry, 64(11), 982-988.

Torrance, N., Elliott, A. M., Lee, A. J., \& Smith, B. H. 2010. Severe chronic pain is associated with increased 10 year mortality. A cohort record linkage study. European journal of pain, 14(4), 380-386. 
Torregrossa, M. M., Jutkiewicz, E. M., Mosberg, H. I., Balboni, G., Watson, S. J., \& Woods, J. H. 2006. Peptidic delta opioid receptor agonists produce antidepressant-like effects in the forced swim test and regulate BDNF mRNA expression in rats. Brain research, 1069(1), 172-181.

Torres, G. E. 2006. The dopamine transporter proteome. Journal of neurochemistry, 97, 3-10.

Townsend, E. A., Naylor, J. E., Negus, S. S., Edwards, S. R., Qureshi, H. N., McLendon, H. W., . . da Silva, F. S. 2017. Effects of nalfurafine on the reinforcing, thermal antinociceptive, and respiratory-depressant effects of oxycodone: modeling an abuse-deterrent opioid analgesic in rats. Psychopharmacology, 234(17), 2597-2605.

Tran-Nguyen, L. T., Fuchs, R. A., Coffey, G. P., Baker, D. A., O’Dell, L. E., \& Neisewander, J. L. 1998. Time-dependent changes in cocaine-seeking behavior and extracellular dopamine levels in the amygdala during cocaine withdrawal. Neuropsychopharmacology, 19(1), 48-59.

Twycross, R. 1997. Cancer pain classification. Acta Anaesthesiologica Scandinavica, 41(1), 141145.

Ueno, Y., Mori, A., \& Yanagita, T. 2013. One year long-term study on abuse liability of nalfurafine in hemodialysis patients. International journal of clinical pharmacology and therapeutics, 51(11), 823-831.

UNODC. (2018). World Drug Report 2018. Vienna, Austria: United Nations Office on Drugs and Crime.

Urban, J. D., Clarke, W. P., Von Zastrow, M., Nichols, D. E., Kobilka, B., Weinstein, H., . . Sexton, P. M. 2007. Functional selectivity and classical concepts of quantitative pharmacology. Journal of Pharmacology and Experimental Therapeutics, 320(1), 1-13.

Valdez, G. R., \& Harshberger, E. 2012. Kappa opioid regulation of anxiety-like behavior during acute ethanol withdrawal. Pharmacology Biochemistry and Behavior, 102(1), 44-47.

van Rijn, R. M., Brissett, D. I., \& Whistler, J. L. 2010. Dual efficacy of delta opioid receptor-selective ligands for ethanol drinking and anxiety. Journal of Pharmacology and Experimental Therapeutics, 335(1), 133-139.

Vanderah, T. W. 2010. Delta and kappa opioid receptors as suitable drug targets for pain. The Clinical journal of pain, 26, S10-S15.

Vanderschuren, L. J., Schoffelmeer, A. N., Wardeh, G., \& De Vries, T. J. 2000. Dissociable effects of the K-opioid receptor agonists bremazocine, U69593, and U50488H on locomotor activity and long-term behavioral sensitization induced by amphetamine and cocaine. Psychopharmacology, 150(1), 35-44.

Varadi, A., Marrone, G. F., Eans, S. O., Ganno, M. L., Subrath, J. J., LeRouzic, V., . . Majumdar, S. 2015. Synthesis and Characterization of a Dual Kappa-Delta Opioid Receptor Agonist Analgesic Blocking Cocaine Reward Behavior. ACS chemical neuroscience.

Váradi, A., Marrone, G. F., Palmer, T. C., Narayan, A., Szabó, M. R., Le Rouzic, V., . . Kalra, S. 2016. Mitragynine/Corynantheidine pseudoindoxyls as opioid analgesics with mu agonism and delta antagonism, which do not recruit $\beta$-Arrestin-2. Journal of medicinal chemistry, 59(18), 8381-8397.

Vaughan, R. A., \& Foster, J. D. 2013. Mechanisms of dopamine transporter regulation in normal and disease states. Trends in pharmacological sciences, 34(9), 489-496.

Velasquez, K. M., Molfese, D. L., \& Salas, R. 2014. The role of the habenula in drug addiction. Frontiers in human neuroscience, 8, 174.

Vergura, R., Balboni, G., Spagnolo, B., Gavioli, E., Lambert, D. G., McDonald, J., . . G Guerrini, R. 2008. Anxiolytic-and antidepressant-like activities of H-Dmt-Tic-NH-CH (CH2-COOH)-Bid (UFP-512), a novel selective delta opioid receptor agonist. Peptides, 29(1), 93-103. 
Viscusi, E. R., Webster, L., Kuss, M., Daniels, S., Bolognese, J. A., Zuckerman, S., . . Skobieranda, F. 2016. A randomized, phase 2 study investigating TRV130, a biased ligand of the $\mu$-opioid receptor, for the intravenous treatment of acute pain. Pain, 157(1), 264-272.

Volkow, N. D., Fowler, J. S., Wang, G. J., Hitzemann, R., Logan, J., Schlyer, D. J., . . . Wolf, A. P. 1993. Decreased dopamine D2 receptor availability is associated with reduced frontal metabolism in cocaine abusers. Synapse, 14(2), 169-177.

Volkow, N. D., Wang, G.-J., Telang, F., Fowler, J. S., Logan, J., Childress, A.-R., . . Wong, C. 2006. Cocaine cues and dopamine in dorsal striatum: mechanism of craving in cocaine addiction. Journal of Neuroscience, 26(24), 6583-6588.

Volkow, N. D., Hitzemann, R., Wang, G. J., Fowler, J. S., Wolf, A. P., Dewey, S. L., \& Handlesman, L. 1992. Long-term frontal brain metabolic changes in cocaine abusers. Synapse, 11(3), 184190.

Volkow, N. D., Fowler, J. S., Wang, G.-J., Swanson, J. M., \& Telang, F. 2007. Dopamine in drug abuse and addiction: results of imaging studies and treatment implications. Archives of neurology, 64(11), 1575-1579.

Vonvoigtlander, P., Lahti, R., \& Ludens, J. 1983. U-50,488: a selective and structurally novel nonMu (kappa) opioid agonist. Journal of Pharmacology and Experimental Therapeutics, 224(1), 7-12.

Vunck, S. A., Snider, S. E., van den Oord, E. J., \& Beardsley, P. M. (2011). The kappa opioid receptor agonist, U50, 488, exacerbates conditioned fear in mice: Federation of American Societies for Experimental Biology.

Waldhoer, M., Fong, J., Jones, R. M., Lunzer, M. M., Sharma, S. K., Kostenis, E., . . Whistler, J. L. 2005. A heterodimer-selective agonist shows in vivo relevance of $G$ protein-coupled receptor dimers. Proceedings of the National Academy of Sciences, 102(25), 9050-9055.

Walf, A. A., \& Frye, C. A. 2007. The use of the elevated plus maze as an assay of anxiety-related behavior in rodents. Nature protocols, 2(2), 322.

Walsh, S. L., Strain, E. C., Abreu, M. E., \& Bigelow, G. E. 2001. Enadoline, a selective kappa opioid agonist: comparison with butorphanol and hydromorphone in humans. Psychopharmacology, 157(2), 151-162.

Wang, Y.-h., Sun, J.-f., Tao, Y.-m., Chi, Z.-q., \& Liu, J.-g. 2010. The role of k-opioid receptor activation in mediating antinociception and addiction. Acta Pharmacologica Sinica, 31(9), 1065-1070.

Wang, Y.-J., Rasakham, K., Huang, P., Chudnovskaya, D., Cowan, A., \& Liu-Chen, L.-Y. 2011. Sex difference in K-opioid receptor (KOPR)-mediated behaviors, brain region KOPR level and KOPR-mediated guanosine 5'-O-(3-[35S] thiotriphosphate) binding in the guinea pig. Journal of Pharmacology and Experimental Therapeutics, 339(2), 438-450.

Wang, Y.-J., Tao, Y.-M., Li, F.-Y., Wang, Y.-H., Xu, X.-J., Chen, J., . . Zhang, A. 2009. Pharmacological Characterization of ATPM [(-)-3-Aminothiazolo [5, 4-b]-N-cyclopropylmethylmorphinan hydrochloride], a Novel Mixed k-Agonist and $\mu$-Agonist/-Antagonist That Attenuates Morphine Antinociceptive Tolerance and Heroin Self-Administration Behavior. Journal of Pharmacology and Experimental Therapeutics, 329(1), 306-313.

Ward, S. J., \& Roberts, D. C. 2007. Microinjection of the $\delta$-opioid receptor selective antagonist naltrindole $5^{\prime}$-isothiocyanate site specifically affects cocaine self-administration in rats responding under a progressive ratio schedule of reinforcement. Behavioural brain research, 182(1), 140-144.

Watson, S. J., Khachaturian, H., Coy, D., Taylor, L., \& Akil, H. 1982. Dynorphin is located throughout the CNS and is often co-localized with alpha-neo-endorphin. Life sciences, 31(16-17), 1773-1776. 
Weaver, B. A. 2014. How Taxol/paclitaxel kills cancer cells. Molecular biology of the cell, 25(18), 2677-2681.

Wee, S., \& Koob, G. F. 2010. The role of the dynorphin-k opioid system in the reinforcing effects of drugs of abuse. Psychopharmacology, 210(2), 121-135.

Weeks, J. R. 1962. Experimental morphine addiction: method for automatic intravenous injections in unrestrained rats. Science, 138(3537), 143-144.

Wenghofer, E. F., Wilson, L., Kahan, M., Sheehan, C., Srivastava, A., Rubin, A., \& Brathwaite, J. 2011. Survey of Ontario primary care physicians' experiences with opioid prescribing. Canadian Family Physician, 57(3), 324-332.

White, K. L., Robinson, J. E., Zhu, H., DiBerto, J. F., Polepally, P. R., Zjawiony, J. K., . . Roth, B. L. 2015. The G Protein-Biased K-Opioid Receptor Agonist RB-64 Is Analgesic with a Unique Spectrum of Activities In Vivo. Journal of Pharmacology and Experimental Therapeutics, 352(1), 98-109.

Wilkins, C., Sweetsur, P., Smart, B., \& Griffiths, R. 2006. Recent trends in illegal drug use in New Zealand, 2006-2010. Findings from the, 2007(2008), 2009.

Williams, D. A., Zheng, Y., David, B. G., Yuan, Y., Zaidi, S. A., Stevens, D. L., . . Akbarali, H. I. 2016. $6 \beta$-N-heterocyclic substituted naltrexamine derivative BNAP: a peripherally selective mixed MOR/KOR ligand. ACS chemical neuroscience, 7(8), 1120-1129.

Wilson, M., Hitomi, M., \& Schuster, C. 1971. Psychomotor stimulant self administration as a function of dosage per injection in the rhesus monkey. Psychopharmacologia, 22(3), 271281.

Wilson, M. C., \& Schuster, C. R. 1972. The effects of chlorpromazine on psychomotor stimulant self-administration in the rhesus monkey. Psychopharmacologia, 26(2), 115-126.

Wise, R. A. 1982. Neuroleptics and operant behavior: the anhedonia hypothesis. Behavioral and brain sciences, 5(1), 39-53.

Wise, R. A. 1985. The anhedonia hypothesis: Mark III. Behavioral and Brain Sciences, 8(1), 178186.

Wise, R. A. 2008. Dopamine and reward: the anhedonia hypothesis 30 years on. Neurotoxicity research, 14(2-3), 169-183.

Wise, R. A. 2009. Roles for nigrostriatal-not just mesocorticolimbic-dopamine in reward and addiction. Trends in neurosciences, 32(10), 517-524.

Wittmann, W., Schunk, E., Rosskothen, I., Gaburro, S., Singewald, N., Herzog, H., \& Schwarzer, C. 2009. Prodynorphin-derived peptides are critical modulators of anxiety and regulate neurochemistry and corticosterone. Neuropsychopharmacology, 34(3), 775.

Woolf, C. J. 2004. Pain: moving from symptom control toward mechanism-specific pharmacologic management. Annals of internal medicine, 140(6), 441-451.

Woolf, C. J. 2010. What is this thing called pain? The Journal of clinical investigation, 120(11), 3742-3744.

Woolf, C. J., Bennett, G. J., Doherty, M., Dubner, R., Kidd, B., Koltzenburg, M., . . Torebjork, E. (1998). Towards a mechanism-based classification of pain? : LWW.

Woolf, C. J., \& Costigan, M. 1999. Transcriptional and posttranslational plasticity and the generation of inflammatory pain. Proceedings of the National Academy of Sciences, 96(14), 7723-7730.

Wu, H., Wacker, D., Mileni, M., Katritch, V., Han, G. W., Vardy, E., . . Carroll, F. I. 2012. Structure of the human K-opioid receptor in complex with JDTic. Nature, 485(7398), 327.

Wu, Q., Reith, M. E., Kuhar, M. J., Carroll, F. I., \& Garris, P. A. 2001. Preferential increases in nucleus accumbens dopamine after systemic cocaine administration are caused by unique characteristics of dopamine neurotransmission. Journal of Neuroscience, 21(16), 63386347. 
Xiao, W., Boroujerdi, A., Bennett, G., \& Luo, Z. 2007. Chemotherapy-evoked painful peripheral neuropathy: analgesic effects of gabapentin and effects on expression of the alpha-2-delta type-1 calcium channel subunit. Neuroscience, 144(2), 714-720.

Xie, Z., Bhushan, R. G., Daniels, D. J., \& Portoghese, P. S. 2005. Interaction of Bivalent Ligand KDN21 with Heterodimeric $\delta$-K Opioid Receptors in HEK293 Cells. Molecular pharmacology.

Xi, Z.-X., Fuller, S. A., \& Stein, E. A. 1998. Dopamine release in the nucleus accumbens during heroin self-administration is modulated by kappa opioid receptors: an in vivo fast-cyclic voltammetry study. Journal of Pharmacology and Experimental Therapeutics, 284(1), 151161.

Yamamoto, T., Ohno, M., \& Ueki, S. 1988. A selective k-opioid agonist, U-50,488 H, blocks the development of tolerance to morphine analgesia in rats. European journal of pharmacology, 156(1), 173-176.

Yan, F., Bikbulatov, R. V., Mocanu, V., Dicheva, N., Parker, C. E., Wetsel, W. C., . . Zjawiony, J. K. 2009. Structure-based design, synthesis, and biochemical and pharmacological characterization of novel salvinorin A analogues as active state probes of the k-opioid receptor. Biochemistry, 48(29), 6898-6908.

Yasuda, K., Raynor, K., Kong, H., Breder, C. D., Takeda, J., Reisine, T., \& Bell, G. I. 1993. Cloning and functional comparison of kappa and delta opioid receptors from mouse brain. Proceedings of the National Academy of Sciences, 90(14), 6736-6740.

Yin, H. H., Ostlund, S. B., \& Balleine, B. W. 2008. Reward-guided learning beyond dopamine in the nucleus accumbens: the integrative functions of cortico-basal ganglia networks. European Journal of Neuroscience, 28(8), 1437-1448.

Yoshida, M., Yokoo, H., Mizoguchi, K., Kawahara, H., Tsuda, A., Nishikawa, T., \& Tanaka, M. 1992. Eating and drinking cause increased dopamine release in the nucleus accumbens and ventral tegmental area in the rat: measurement by in vivo microdialysis. Neuroscience letters, 139(1), 73-76.

Zan, G.-Y., Wang, Q., Wang, Y.-J., Chen, J.-C., Wu, X., Yang, C.-H., . . Hu, X.-W. 2016. p38 mitogenactivated protein kinase activation in amygdala mediates $\mathrm{K}$ opioid receptor agonist U50, $488 \mathrm{H}$-induced conditioned place aversion. Neuroscience, 320, 122-128.

Zapata, A., Kivell, B., Han, Y., Javitch, J. A., Bolan, E. A., Kuraguntla, D., . . Samuvel, D. J. 2007. Regulation of dopamine transporter function and cell surface expression by D3 dopamine receptors. Journal of Biological Chemistry, 282(49), 35842-35854.

Zhang, L.-s., Wang, J., Chen, J.-c., Tao, Y.-m., Wang, Y.-h., Xu, X.-j., . . Hu, X.-w. 2015. Novel kopioid receptor agonist MB-1C-OH produces potent analgesia with less depression and sedation. Acta Pharmacologica Sinica, 36(5), 565.

Zhang, L., Elmer, L. W., \& Little, K. Y. 1998. Expression and regulation of the human dopamine transporter in a neuronal cell line. Molecular brain research, 59(1), 66-73.

Zhang, X., Bao, L., \& Guan, J.-S. 2006. Role of delivery and trafficking of $\delta$-opioid peptide receptors in opioid analgesia and tolerance. Trends in pharmacological sciences, 27(6), 324-329.

Zhang, Y., Butelman, E. R., Schlussman, S. D., Ho, A., \& Kreek, M. J. 2004a. Effect of the endogenous $\mathrm{K}$ opioid agonist dynorphin $\mathrm{A}$ (1-17) on cocaine-evoked increases in striatal dopamine levels and cocaine-induced place preference in C57BL/6J mice. Psychopharmacology, 172(4), 422-429.

Zhang, Y., Butelman, E. R., Schlussman, S. D., Ho, A., \& Kreek, M. J. 2004b. Effect of the $\mathrm{k}$ opioid agonist R-84760 on cocaine-induced increases in striatal dopamine levels and cocaineinduced place preference in C57BL/6J mice. Psychopharmacology, 173(1-2), 146-152. 
Zhang, Y., Butelman, E. R., Schlussman, S. D., Ho, A., \& Kreek, M. J. 2005. Effects of the plantderived hallucinogen salvinorin $A$ on basal dopamine levels in the caudate putamen and in a conditioned place aversion assay in mice: agonist actions at kappa opioid receptors. Psychopharmacology, 179(3), 551-558.

Zhou, Y., Leri, F., Grella, S. L., Aldrich, J. V., \& Kreek, M. J. 2013. Involvement of dynorphin and kappa opioid receptor in yohimbine-induced reinstatement of heroin seeking in rats. Synapse, 67(6), 358-361.

Zhu, J., Chen, C., Xue, J.-C., Kunapuli, S., DeRiel, J. K., \& Liu-Chen, L.-Y. 1995. Cloning of a human K opioid receptor from the brain. Life sciences, 56(9), PL201-PL207.

Zielińska, M., Jarmuż, A., Sałaga, M., Lipkowski, A. W., \& Fichna, J. 2016. Mixed MOP/DOP agonist biphalin elicits anti-transit effect in mouse models mimicking diarrhea-predominant irritable bowel syndrome symptoms. Pharmacological reports, 68(1), 32-36.

Zimmer, L., Kodas, E., Guilloteau, D., Garreau, L., Besnard, J.-C., \& Chalon, S. 2000. Microdialysis as a tool for in vivo study of dopamine transporter function in rat brains. Journal of neuroscience methods, 103(2), 137-144. 